\title{
Plant virus identification and virus-vector-host interactions
}

Yahya Zakaria Abdou Gaafar

ORCID

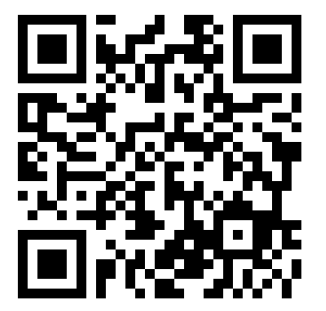

0000-0002-7833-1542 



\title{
Plant virus identification and virus- vector-host interactions
}

\author{
Dissertation \\ for the award of the degree \\ "Doctor rerum naturalium" (Dr.rer.nat.) \\ "Doctor of Philosophy" Ph.D. Division of Mathematics and Natural Sciences \\ of the Georg-August-Universität Göttingen \\ within the International PhD Programme for Agricultural Sciences in Göttingen (IPAG) \\ of the Graduate School Forest and Agricultural Sciences (GFA)
}

submitted by

Yahya Zakaria Abdou Gaafar

from Cairo, Egypt

Göttingen, 2019 


\title{
Thesis committee
}

\author{
Prof. Dr. Stefan Vidal
}

Georg-August University Göttingen, Department for Crop Sciences, Agricultural Entomology

Prof. Dr. Edgar Maiss

Leibniz Universität Hannover, The Institute of Horticultural Production Systems, Section of Phytomedicine

Dr. Heiko Ziebell

Julius Kühn-Institut (JKI), Federal Research Institute for Cultivated Plants, Institute for Epidemiology and Plant Diagnostics

\section{Members of the Examination Board Reviewer}

\section{Prof. Dr. Michael Rostás}

Georg-August University Göttingen, Department for Crop Sciences, Agricultural Entomology 
Plant virus identification and virus-vector-host interactions

Copyright @ 2019 by Yahya Zakaria Abdou Gaafar.

All rights reserved.

Printed in Germany.

No part of this book may be used or reproduced in any manner whatsoever without written permission except in the case of brief quotations embodied in critical articles or reviews.

For information contact; yahyaz.a.gaafar@gmail.com

Book layout and cover were designed by the author.

November 2019 
Dedicated to my family

IV | P a g e 


\section{Contents}

Chapter 1: General introduction __ 1

Part one: Plant virus identification __ 13

Chapter 2: Plant disease aetiology__ 15

Chapter 3: Investigating the pea virome in Germany - old friends and new players in the field(s) __ 88

Part two: Virus-vector-host interactions __ 161

Chapter 5: Aphid transmission of nanoviruses: a review

Chapter 6: Probing and feeding behaviours of Acyrthosiphon pisum change on nanoviruses-infected faba beans

Chapter 7: General discussion 192

Summary 199

References

Acknowledgments

Curriculum vitae 





\section{Chapter 1: General introduction}

\subsection{Plant viruses}

Plant viruses are the cause of many crop diseases worldwide, leading to both yield and quality losses e.g., reduction in growth, vigour and market value (Bos, 1982; Hull, 2014). The type and severity of the host reactions to virus infections are very variable (Hull, 2014). They depend greatly on the virus strains, sources of infection, the time of infection, the crop genotypes and also influenced by environmental conditions (Hull, 2014). Several plant viruses are highly contagious and their effects on plants are often drastic. The losses caused by plant virus infections can have severe financial implications or have socio-economic effects (Anderson et al., 2004; Hull, 2009; Patil et al., 2015; Pechinger et al., 2019).

Virus infection can cause histological changes to the cells and the intracellular structure (Hull, 2009). The symptoms caused by virus infection vary from necrotic or chlorotic lesions on inoculated leaves to systemic e.g., mosaic, mottle, stunting and leaves distortions (Hull, 2014). Nevertheless, some virus infections cause mild or no symptoms. Infection agents e.g., viroids and phytoplasma can induce diseases that resemble virus infections (Hull, 2014). Also, virus associated nucleic acids can alter the disease symptoms (Roossinck et al., 1992; Ziebell \& Carr, 2010). Some virus-like symptoms e.g., yellowing and necrosis, can easily be confused with non-viral disorders. Additionally, complex or multiple infections in plants are very common (Bos, 1982; Al Rwahnih et al., 2009; Carvajal-Yepes et al., 2014). These can consist of different viruses, or viruses with other pathogens, pests or abiotic factors. Such infections generally alter the plant physiology and in consequence the susceptibility and sensitivity to other infecting agents in an additive or synergistic effect or non-additive effect resulting in changes in the displayed symptoms (Bos, 1982; Syller, 2012). Furthermore, there are no simple relationships between virus content within a plant or virus incidence within a crop and the yield losses (Bos, 1982). Thus, detecting and identifying the exact virus causing the disease can be difficult.

However, determining the exact disease causative agent (known viruses, new viruses or virus strains) is necessary to decide which management strategy (e.g., insect vector control, resistance breeding, provision of virus-free germplasm etc) could be applied. In addition, it is important to detect quarantine viruses and prevent them from entering a country and becoming established. Therefore, it helps also in deciding on monitoring and preventive strategies, and in the prediction of plant diseases in annual crops.

\subsection{Plant virus diagnostics:}

Plant virus diagnosis often starts with spotting suspicious plants with "virus-like" symptoms in a field or greenhouse and sending it to diagnostics laboratories for analysis. 
A range of techniques are available to detect and confirm the aetiology of the disease (Hamilton et al., 1981; Hull, 2009). Important factors are taken in consideration when choosing the detection methods i.e., the sensitivity of the method to small amounts of viruses, accuracy, reproducibility, cost, time required, level of expertise needed and ability to perform in field (Hull, 2009). Additionally, the choice of test will determine the outcome i.e., whether only a virus family can be determined or a virus species or if strain-specific detection is possible.

\subsubsection{Conventional detection methods:}

Conventional detection methods have been developed and successfully implemented for a long time as virus detection tools and are widely used in many laboratories. Virus diagnosis has been relying on experienced specialists who can recognize and describe the disease causal agent from the symptoms on hosts, complemented with methods e.g., bioassays on indicator plants and electron microscopy (Boonham et al., 2014). Conventional methods include bioassays, electron microscopy (EM), enzyme-linked immunosorbent assay (ELISA), Western, Northern and Southern blotting and polymerase chain reaction (PCR)-based methods. They provide rapid and inexpensive diagnoses for known viruses and viroids (Wu et al., 2015). The following are descriptions of the commonly used methods in virus/viroid diagnosis.

\subsubsection{Bioassays}

Indicator plants have been used from the early ages of plant virology for propagation of plant viruses. Based on symptoms and host range reactions, differential host plants were used for the identification and classification of a number of plant viruses (Kirby et al., 2001; Hull, 2014). However, a correct diagnosis based on symptoms is not possible and these days indicator plants are mainly used for virus propagation and enrichment for the subsequent use in different tests (e.g., electron microscopy).

\subsubsection{Electron microscopy}

Due to the small size of plant viruses, EM is the only technology that can directly visualise virus particles. The high resolution power of EM provides direct images at nanometre scale for virus diagnosis and research (Richert-Pöggeler et al., 2018). Transmission electron microscopy (TEM) can be used as initial step in virus diagnosis from crud plant extracts without the necessity of viral enrichment (Bawden \& Nixon, 1951; Gentile \& Gelderblom, 2014; Richert-Pöggeler et al., 2018). A main advantage of EM for viral diagnosis is that it does not require virus-specific reagents thus it provides an open view on the sample (Goldsmith \& Miller, 2009). EM may not be able to identify a virus beyond the family level, thus additional assays can be performed which require virusspecific reagents e.g., antibodies. Immunosorbent electron microscopy (ISEM), as an example, increases the sensitivity of EM by virus trapping (Debrick, 1973; Roberts \& Harrison, 1979). If specific antibodies are available, they can be used to "decorate" the virus particles and therefore differentiate between different species depending on the specificity of these antibodies. However, not always are specific antibodies available for all viruses. Additionally, an enrichment step is required for phloem restricted and low titre viruses, viruses without virions and viroids e.g., ultracentrifugation (Richert-Pöggeler et 
al., 2018). Moreover, EM requires expertise, and EM facilities are not widely available (Naidu \& Hughes, 2003). Thus, other methods such as serological or molecular assays can be performed additionally for specific virus identification and characterisation e.g., ELISA and PCR-based methods.

\subsubsection{Enzyme-linked immunosorbent assay}

The establishment of the ELISA assay was a revolution in virus diagnostics by simplifying virus detection and shortening the time required to reach conclusive results (Clark \& Adams, 1977; Torrance \& Jones, 1981). ELISA assays target proteins e.g., viral coat and movement proteins by antibodies. As a routine virus diagnostics test, ELISA is easy to use, cost effective, robust and scalable (Casper \& Meyer, 1981; Torrance \& Jones, 1981; Koenig \& Paul, 1982). However, it requires the production of high-quality antisera which requires viral protein purification and expertise which can be lengthy procedure (Boonham et al., 2014). Moreover, the antisera are often lack the sensitivity to correctly identify closely related virus strains, and in several cases it is not possible to differentiate viruses from the same genus due to cross-reactivity of antisera (Boonham et al., 2014). ELISA is also difficult to use for multiple-target detection from one sample, as several tests need to be set up (Boonham et al., 2014).

\subsubsection{PCR-based methods}

PCR-based methods e.g., classical PCR, reverse-transcription (RT)-PCR and realtime (or quantitative RCR [qPCR]) have been used for the diagnosis of plant viruses and viroids, and many assays have been published (Boonham et al., 2014). PCR-based methods target nucleic acid sequences by primers. They require a reliable nucleic acid extraction method and sequence information of the viruses for primer design. PCR-based methods can be sensitive, inexpensive and require minimal skill to be performed. They have been used for plant virus detection since early 1990s (Vunsh et al., 1990). PCR multiplexing allows the detection of multiple species or strains in a single reaction by combining specific primers for different viruses (Webster et al., 2004). The specificity of PCR-based methods depends on the design of proper primers that are unique to the target virus/viroid. Moreover, virus-specific primers or probes can detect virus up to limited sequence variation, however new viruses, strains or divergent isolates will not be detected.

In general, molecular or serological testing are targeted methods which means they are limited to detecting the knowns. Additionally, in cases of mixed infections, such methods would likely miss the other disease causal agent. Therefore, virology diagnosticians need additional tools for diagnosing the unknowns and the variants.

\subsubsection{Sequencing}

\subsubsection{Sanger chain termination}

The Sanger chain termination method was developed in 1977 (Sanger et al., 1977). This method uses labelled dideoxynucleotide (ddATP, ddGTP, ddCTP, or ddTTP) in four separated sequencing reactions which terminate DNA synthesis upon incorporation. The 
generated sequences are then denatured and visualised by gel electrophoresis. In virus diagnostics, Sanger sequencing is commonly used to sequence PCR amplicons directly (Bernad \& Duran-Vila, 2006; Hoang et al., 2011). When multiple PCR amplicons are present in one reaction or sequence variation within one amplicon is suspected, PCR products can be cloned in bacterial cells, followed by selection and propagation of bacterial colonies followed by plasmids purification and Sanger sequencing. These sequences can be used for bioinformatic analysis and comparison with sequences available in public databases. Another application when using generic primers for the identification of more than one virus from the same genus or family (Abraham et al., 2007). For unknown virus identification, a random-PCR method (rPCR) to construct whole cDNA library from sample RNA or dsRNA extracts, or library from rolling circle amplification (RCA) for circular DNA and enzymatic fragmentation are performed (Dodds et al., 1984; Haan et al., 1989; Froussard, 1992; Johne et al., 2009). These approaches helped in the detection of many virus including new ones.

For long time, a typical Sanger sequencing reaction included the use of radioisotopes and other harsh chemicals (Wu et al., 2015). This sequencing method was a labour-intensive process and only determined few hundred nucleotides (nt) at a time (Kircher \& Kelso, 2010). With the development of capillary array electrophoresis and other detection systems, the production of 96-channel capillary HTS sequencers was possible e.g., 3730xI DNA Analyzer (Applied Biosystems, Inc) (Kambara \& Takahashi, 1993; Kircher \& Kelso, 2010). Such sequencer yield 96 or 384 sequences of about 600 to 1,000 nt per run and a maximum of about $1.5 \mathrm{Mb}$ sequences per day with single-pass error rate of 0.1 to $1 \%$ (Wu et al., 2015).

\subsubsection{High-throughput sequencing}

Over 15 years ago high-throughput sequencing (HTS), formerly known as nextgeneration sequencing (NGS) appeared on the market and revolutionized sequencing capabilities (van Dijk et al., 2014a). It enabled parallel sequencing of millions of nucleic acid sequences in short time for comparatively low cost. Since then, a lot of progress has been made in read length, speed, throughput, and in costs reduction (van Dijk et al., 2014a). These advances paved the way for the development of novel HTS applications in life sciences such as in diagnostics and metagenomics (Roossinck et al., 2010; Roossinck et al., 2015).

HTS was first applied for plant virus identification in 2009 using different preparation approaches and different sequencing platforms (Adams et al., 2009; Al Rwahnih et al., 2009; Kreuze et al., 2009). It has proven very successful for virus discovery to resolve the disease aetiology in many agricultural crops (Roossinck et al., 2015). It enables the simultaneous sequencing of total nucleic acid content of a sample, and thus detection, of any organism present in this sample. HTS carries the promise of generic and routine tool for virus detection. However, several steps need to be taken into consideration when applying HTS technologies: a) nucleic acid extraction and virus sequences enrichment; b) library preparation; c) automated sequencing; d) data analysis. 


\section{a) Nucleic acid extraction and virus sequences enrichment:}

Viruses possess different genomes i.e., DNA (single or double stranded, circular or linear) or RNA (single [negative or positive sense] or double stranded, circular or linear) (Hull, 2009). Additionally, viroids possess circular single stranded RNA. Total DNA, total RNA, double stranded RNA (dsRNA) or small RNA (sRNA) extraction can be used for HTS detection of plant viruses and viroids. Therefore, many extraction protocols are available depending on the target nucleic acid.

For plant virus detection by HTS, virus sequence enrichment is required prior to sequencing to relatively increase the virus sequences in comparison to the host sequences (Wu et al., 2015). Although the sizes of the virus/viroid genomes are small in comparison to other organisms, to achieve full genome coverage, the number of virus sequence reads must be high in order to trace their sequences that can be undetectable within the host overrepresented sequences which is challenging for bioinformatic analysis (Wu et al., 2015; Adams \& Fox, 2016). Additionally, there is no single reference gene or marker sequence shared by all viruses that could be used for virus identification as in case of other organisms such as fungi or bacteria where the internal transcribed spacer (ITS) or 16S ribosomal RNA can be used for general detection of these pathogens (Leff et al., 2017).

There are different methods available for virus enrichment with the most common ones dsRNA, ribosomal RNA ( $r R N A$ ) depletion, rolling circle amplification (RCA) and sRNA enrichments (Dodds et al., 1984; Kreuze et al., 2009; Roossinck et al., 2010; Idris et al., 2014; Knierim et al., 2017). Each enrichment method has its advantages and disadvantages i.e., some are time consuming, some require large amounts of sample starting material, others may cause bias in the detection (not suitable for all viruses) (Wu et al., 2015; Visser et al., 2016; Pecman et al., 2017).

The extraction and analysis of dsRNA has been used for plant virus detection for a long time (Morris, 1979; Tzanetakis \& Martin, 2008; Okada et al., 2015). dsRNA is produced as an intermediate during replication of RNA viruses and viroids and not "naturally" occurring in plants (Wu et al., 2015). Extraction of dsRNA molecules is relatively easy, and the molecules are quite stable. Al Rwahnih and colleague compared HTS sequences derived from dsRNA or total RNA extracts from the same plant samples and found that virus reads increased from $2 \%$ to $53 \%$ with dsRNA enrichment (AI Rwahnih et al., 2009). However, not all RNA viruses accumulate high concentrations of dsRNA, and DNA viruses have a different mode of replication, but few DNA virus sequences could also be recovered ( $\mathrm{Wu}$ et al., 2015). Depending on the dsRNA extraction method, high amounts of plant material may be required, other protocols are time consuming, or requiring an extra amplification step (Roossinck et al., 2010; Romanovskaya et al., 2013; Blouin et al., 2016).

rRNA depletion of total RNA is useful for reducing the host sequences by removing the majority of the rRNAs which are highly abundant from the plant sample before further preparations (Adams \& Fox, 2016). This can result in a 10-fold virus RNA enrichment (Adams \& Fox, 2016). An additional step may be applied by selecting poly (A) tailed RNAs 
(Visser et al., 2016). However, this additional step is not useful for the identification of viruses that do not possess poly $(A)$ tails.

Plants produce virus-derived small interfering RNAs (vsiRNAs) in response to virus infection (Ding \& Lu, 2011). Moreover, the dsRNA replicative intermediates produced during the replication of viruses and viriods can also be processed into small interfering RNA in plants (Ding, 2010). Thus, all replicating viruses and viroids in a diseased plant can be detected by SRNA extraction. sRNA extraction by gel purification requires long time (up to two weeks). Nevertheless, a new extraction method was developed which can be performed within a day (Li et al., 2013).

RCA approach takes the advantage of Phi 29 DNA polymerase and amplify circular DNA molecules in a given sample (Dean et al., 2001; Idris et al., 2014). Thus, RNA and noncircular DNA viruses cannot be amplified with this approach. Moreover, the Phi 29 polymerase may induce errors at early stages of amplification.

It is therefore desired to identify a generic approach in which it is possible to detect and identify all viruses and viroids with different genomes that can be easily used in diagnostic laboratories.

\section{b) Library preparation:}

The quality of sequencing data depends on the quality of the sequenced material. Thus, the library preparation must guarantee low bias and high complexity in order to achieve the most genomic coverage (van Dijk et al., 2014b). Many library preparation protocols are available and most of them are compatible with the Illumina system (described later) (van Dijk et al., 2014a; van Dijk et al., 2014b).

General steps for library preparation i.e., the fragmentation of the nucleic acid, reverse transcription of RNA and dscDNA synthesis (when RNA is the starting material), adaptors and barcodes/indexes ligation, and with or without size selection and amplification (van Dijk et al., 2014b). The library design may allow sequencing of both strands of DNA which increases accuracy. For single-molecule sequencing platforms (described later), the library preparation is minimal, where it involves template fragmentation, adapters ligation with or without amplification step (van Dijk et al., 2014a). The choice of the protocol depends on the platform on which the sequencing will be run, and the performed study.

\section{c) Sequencing platforms:}

Roche 454 system was the first commercially HTS platform (Liu et al., 2012). This platform uses the pyrosequencing technology and emulsion PCR as amplification approach (Margulies et al., 2005). It produced initially 100-150 bp of sequence lengths and improved to $1 \mathrm{~kb}$ but with relatively low throughput (700 Mb per run), high error rates and high reagent cost (Metzker, 2010). Now the production of 454 is shut down and no more supporting by the company since 2016 (van Dijk et al., 2014a). Following 454, several platforms were developed including Solexa/Illumina, SOLiD, Ion Torrent, PacBio and MinION platforms. 
The Solexa/lllumina GA platform adopted sequencing by synthesis (SBS) technology and generated larger number (1Gb per run) but shorter reads compared to 454 platform (Liu et al., 2012). Illumina SBS uses a proprietary reversible terminator-based method that detects single bases as they are incorporated into DNA template strands. Prior to sequencing, clonal clusters of amplified DNA fragments are generated through bridge amplification. Sequencing then starts with sequencing reagents containing a polymerase and fluorescently labelled nucleotides. Each nucleotide base is added, and the flow cell is photographed and the emission from each cluster is recorded. Each base is identified by the intensity and wavelength of the emission. This cycle is repeated to create the read length specified. Illumina SBS technology results in highly accurate base-by-base sequencing compared to other technologies. With the improvements in polymerases, buffers, flowcells, and software, several platforms from Illumina are currently available e.g., HiSeq, MiSeq and NextSeq. The size of the generated reads, their quality and amounts vary from device to another (Liu et al., 2012; Reuter et al., 2015).

The HiSeq and MiSeq platforms are the most established sequencers (Reuter et al., 2015). MiSeq is a fast, benchtop sequencer, generates reads of $300 \mathrm{bp}$ in length and up to $15 \mathrm{~Gb}$ per run (Illumina). HiSeq produces maximum read length of $150 \mathrm{bp}$ and over $1 \mathrm{~Tb}$ per run (Illumina). NextSeq produces up $120 \mathrm{~Gb}$ per run and maximum read length of $150 \mathrm{bp}$ (Illumina). Their error rate of Illumina platforms is $<1 \%$ and the quality of the generated reads decreases with increasing the read length (Liu et al., 2012; Reuter et al., 2015). In general, Illumina platforms offer the highest throughput and the lowest cost perbase compare to other platforms (Liu et al., 2012).

Sequencing by Oligo Ligation Detection (SOLiD) by Applied Biosystems uses Ligation-based sequencing technology and emulsion PCR for amplification (Mardis, 2008). On a SOLiD flowcell, the libraries are sequenced by 8 base-probes ligation which contains ligation site, cleavage site and fluorescent dyes. The fluorescent signals are recorded during the probes binding to the template strand then cleaved (Mardis, 2008). The cycle is repeated four times using ladder primer sets. SOLiD initially generated average read length of 25-35 bp ( $3 \mathrm{~Gb}$ data per run) and could reach a high accuracy of $99.85 \%$ after filtering (Mardis, 2008). Later other SOLiD sequencing systems were released with improvements in read length, data output and accuracy (Liu et al., 2012). SOLiD 5500xl, for example, generates reads with 85 bp length (30 Gb per run) and 99.99\% accuracy (Liu et al., 2012).

Ion Torrent Personal Genome Machine (PGM) uses semiconductor sequencing technology (Flusberg et al., 2010). PGM detects the changes in $\mathrm{pH}$ induced by the release of hydrogen ions with the incorporation of each nucleotide (Rothberg et al., 2011). It does not require fluorescence and camera scanning, resulting in higher speed, lower cost, and smaller instrument size (Liu et al., 2012). PGM generates reads with up to 400 bp length (1 Gb per run) with high error rates in homopolymers and insertions and deletions (Liu et al., 2012; van Dijk et al., 2014b). Ion Proton, the second Ion Torrent platform, increased the output compared to the PGM with $10 \mathrm{~Gb}$ per run and maximum read length was $200 \mathrm{bp}$ (Reuter et al., 2015). 
Pacific Bioscience (PacBio) produced the single-molecule real-time (SMRT) sequencing platform. In this technology the clonal amplification is avoided, allowing direct sequencing of the DNA (Reuter et al., 2015). The sequencing is performed with continuous polymerisation of the template in a zero-mode waveguides (ZMWs) with a single polymerase is positioned at its bottom and the presence of labelled nucleotides. With each incorporated base, fluorescent signals are captured in a video in real-time (Levene et al., 2003; Eid et al., 2009). It produces very long reads (60 kb; with $>14 \mathrm{~kb}$ average read lengths) about 50k reads (up to $1 \mathrm{~Gb}$ of data in $4 \mathrm{hr}$ ). However, it has high error rates (about 11\%) which are dominated by indels (Reuter et al., 2015).

MinION nanopore sequencer is a single-molecule sequencing platform produced by Oxford Nanopore Technologies (ONT) (Ip et al., 2015). On the membrane with nanopores "transmembrane proteins with nanoscale pore" incorporated, a voltage can be applied to drive DNA through the pore and an ion current flow can be measured. When a DNA molecule passes through the nanopore, a change of the current in pattern or magnitude can be observed and characterised. The nanopore can then discriminate individual nucleotides by measuring the change in electrical conductivity as DNA molecules pass through the pore (Lu et al., 2016). The current in the nanopore is measured by a sensor several thousand times per second, and the data streams are passed to a microchip called the application-specific integrated circuit (ASIC) (Lu et al., 2016). Finally, data processing is carried out by the MinKNOW software, which deals with data acquisition and analysis (Lu et al., 2016).

The MinION device attracted considerable interest by scientists particularly in the fields of pathogen surveillance and diagnostics applications. MinION is the smallest sequencing device available, portable, and can produce sequence data in real-time (theoretically) (Lu et al., 2016). It can be powered by the Universal Serial Bus (USB) ports of a computer with low hardware requirements and simple configurations but portable devices to operate MinION are now being offered by the ONT company. The main advantage of MinION sequencing is that it can generate longer reads of up to $100 \mathrm{~Kb}$ ( $\mathrm{Lu}$ et al., 2016). Additionally, the device has a low capital cost and its sequencing is cheap (Lu et al., 2016). However, it has rather higher error rates (up 45\%) (Lu et al., 2016).

\section{d) Data analysis:}

Bioinformatic analysis is a crucial step in virus detection using HTS. There are several commercial software e.g., Geneious Prime and CLC genomic workbench, and open platforms such as Galaxy which can be used for HTS data analysis (Massart et al., 2014). Automated bioinformatic pipelines for viruses detection are also available e.g., VirusDetect and Virtool (Rott et al., 2017; Zheng et al., 2017). The high efficiency and sensitivity of the different analysis pipelines in detecting known and novel virus are variable. Several available tools are designed for the detection of certain viruses using mapping to reference or for certain enrichment approach or for certain sequencing platforms e.g., Paparazzi (Vodovar et al., 2011). 
In general, the raw data generated from the sequencing platforms are then subjected to quality trimming to remove the adaptor sequences and low-quality nucleotides (Fig. 1) (Ho \& Tzanetakis, 2014). This is followed by two ways based on the experimental design and the reason for sequencing. If the virus in the sample is known, the reference genome of the virus can be used for mapping the virus reads (Ho \& Tzanetakis, 2014). However, when the cause of the disease is unknown, a de novo assembly is required (Zheng et al., 2017). This is followed by searching the nucleotide and protein databases using Basic Local Alignment Search Tool (BLAST) to detect the closest sequences to the searched reads. Removing the host sequences by mapping to the host genome can be used to reduce the number of reads for the following analyses (Zheng et al., 2017).

Massart and colleagues identified four crucial factors influencing the sensitivity of the bioinformatic analysis for virus/viroid detection i.e., the abundance of virus reads and the novelty of the virus, the assembly and annotation parameters, the completeness of the reference databases and the expertise in results interpretation (Massart et al., 2019). The bioinformatic tools for HTS data analysis are under constant development and these efforts will improve, facilitate and speed up the application of HTS as a diagnostics tool in laboratories.

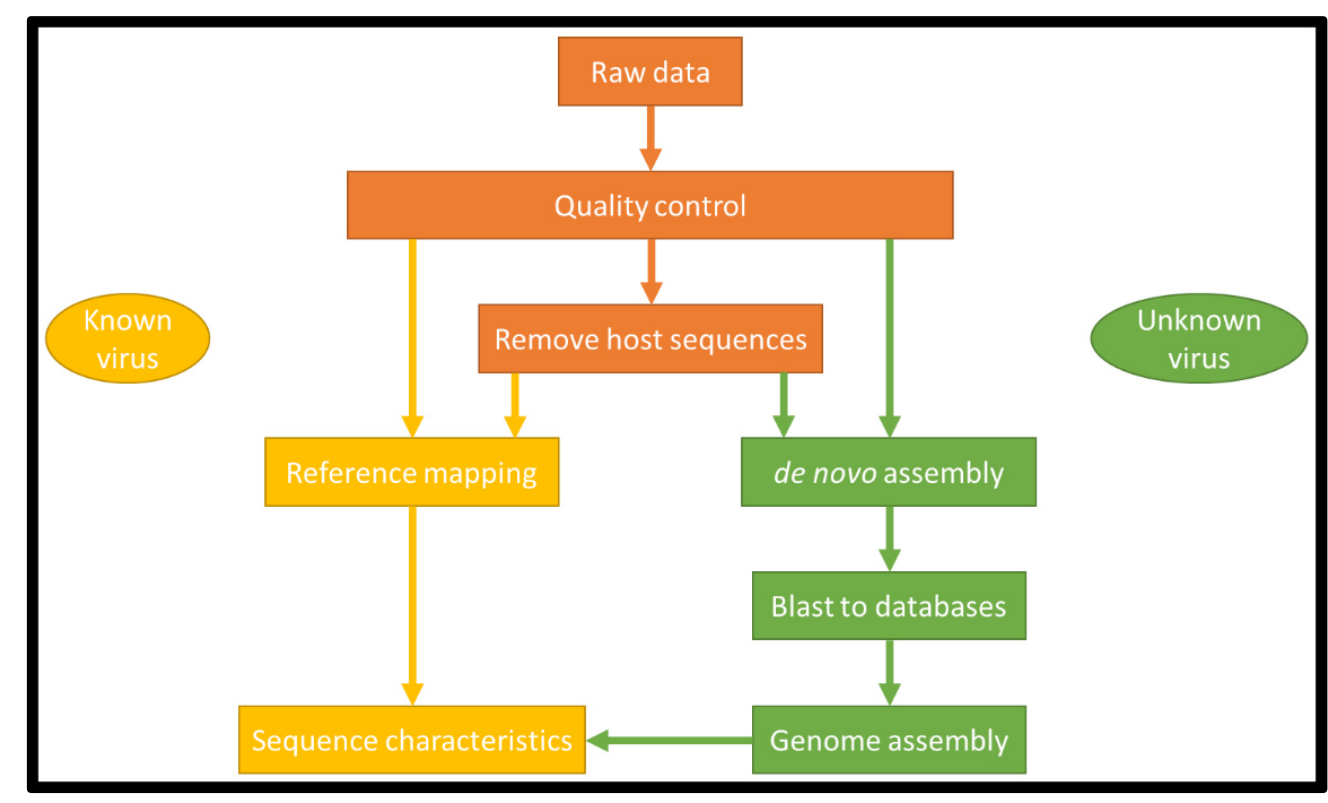

Figure 1: A diagram of two general strategies for HTS data analysis for known and unknown viruses 


\subsection{Plant virus transmission}

Plant viruses possess many routes of transmission; they can be transmitted mechanically, by pollen, seeds or vectors such as nematodes, aphids and other insects. Aphids (Hemiptera, Aphididae) are the most common vector of plant viruses with fifty percent of the insect-vectored plant viruses are transmitted by aphids (Nault, 1997). The successful transport and transmission of viruses may be relying on aphids' high reproduction rates and their ability to spread for long distances.

Plant viruses are transmitted by aphids via different transmission modes depending on the aphid and the virus species: circulative or non-circulative, persistent, semi-persistent or non-persistent, propagative or non-propagative manners (Watson \& Roberts, 1939; Sylvester, 1956; Harris, 1977; Ng \& Perry, 2004; Ng \& Falk, 2006). Depending on the mode of transmission, different strategies for preventing the spread of plant viruses need to be taken into consideration.

Many of the aphid-plant interactions take place at the cellular and tissue levels, especially as a virus vector (Collar et al., 1997). As aphids are phloem feeders, acceptance of the phloem sap is an is required for aphid plant selection (Tjallingii, 1994). Changes in the chemical composition and physical structure of the plant tissues can affect the probing and feeding behaviours of the aphids (Guo et al., 2014). Studying the aphid probing and feeding will reveal more details on the events involved in the transmission of plant viruses.

Electrical penetration graphs (EPG) have contributed substantially to the current knowledge of stylet penetration events in the plant tissue (Gabrys et al., 1997; Pescod et al., 2007; Brunissen et al., 2009). Studying the aphid stylet penetration by electrical recording started with the $A C$ system by Mclean and Kinsey and was further developed by Tjallingii (Mclean \& Kinsey, 1964, 1965; Tjallingii, 1978, 1988). By attaching a gold wire electrode to the dorsum of the aphid and inserting the other electrode in the soil near to the plant root, once the aphid stylet penetrated the plant tissue the electrical circuit is completed (Tjallingii, 1985). The activity of the stylet in the plant tissue can then be recorded as waveforms. Each waveform is correlated to a certain stylet activity (Tjallingii, 1985).

EPG contributed to our understanding of virus transmission. Studies showed that plant virus infection can affect the behaviours and fitness of their vector (Castle \& Berger, 1993; Eigenbrode et al., 2002; Ziebell et al., 2011). These effects are suggested to be related to the virus mode of transmission. Potato leafroll virus, a persistent transmitted circulative virus, enhanced the feeding behaviour of their aphid vector Myzus persicae Sulzer. Only after PLRV-infected potatoes showed visual symptoms, the aphids displayed a lower number of short probing periods before the first phloem activity and lower number and shorter total duration of derailed stylet mechanic (Alvarez et al., 2007). Moreover, PLRV infection improved the aphid fitness by enhancing the aphids acceptance to the infected plant, making it more preferred and attractive for the aphid (Castle \& Berger, 1993; Srinivasan \& Alvarez, 2007). No effects were observed in case of potato virus 
$X$, which is a mechanically transmissible and transmitted independently from insect vectors (Castle et al., 1998; Eigenbrode et al., 2002; Alvarez et al., 2007; Srinivasan \& Alvarez, 2007). In case of non-persistent virus (less vector-dependent) i.e., potato virus $Y$, it induced various effects on the behaviour of the vector depending on the species e.g., it enhanced the growth of $M$. persicae whereas it had no effect on Macrosiphum euphorbiae (Srinivasan \& Alvarez, 2007; Boquel et al., 2011).

In this thesis, the model system nanovirus-Acyrthosiphon pisum-Vicia faba was investigated further. Nanoviruses (members of the genus Nanovirus; family Nanoviridae) are multipartite viruses with at least eight circular ssDNA (Vetten et al., 2011). They are considered a threat to important crops such as legumes (Vetten et al., 2011). They are transmitted in circulative, non-propagative manner by various aphid species e.g., A. pisum and Aphis craccivora (Vetten et al., 2011). The interactions between nanoviruses and their vectors is reviewed in detailed in chapter 5 .

Nanoviruses are interesting model viruses as each genomic component encodes one protein, some of which with unknown functions (Vetten et al., 2011). The availability of infectious clones for each component helped in understanding the role of each virus protein (Timchenko et al., 1999; Timchenko et al., 2000; Timchenko et al., 2006; Grigoras et al., 2009). Grigoras and colleagues found that the nuclear shuttle protein (NSP) encoded by component DNA-N is essential for aphid transmission (Grigoras et al., 2018). The exact role of NSP in transmission is currently unknown. Nevertheless, this protein was found to be interacting with the stress granule component G3BP, the master replicase encoded by DNA-R and interacts with other NSPs in infected plants (Krapp et al., 2017; Krenz et al., 2017).

For faba bean necrotic yellows virus, a different nanovirus, a shorter plant access time is required for the inoculation minimum inoculation access period than for the acquisition ranging from 5 and $15 \mathrm{~min}$ and 15 to 30 minutes, respectively, by both $A$. craccivora and $A$. pisum (Franz et al., 1998). Phloem restricted plant viruses, such as nanoviruses, need to be ingested from the phloem cells in order to be transmitted by translocated through the gut, haemolymph and to the saliva for new infections. We expect a close relationship between the E2 waveforms (correlated to ingestion) in EPGs and nanoviruses acquisition. This could be experimentally confirmed for another circulative virus i.e., barely yellow dwarf virus (Prado \& Tjallingii, 1994). The amount of acquired virus particles and proteins is expected to increase with the increase the E2 time. Whether the presence of nanovirus changes the behaviour as it is expected that nanovirus interacts with the aphids' body upon acquisition, or to change the host making it favourable by the vector, is currently unknown. Whether the absence of the NSP may also affect the behaviour is also unknown. 


\subsection{Aim and scope of the thesis:}

- To identify the aetiology of several plant diseases using conventional and HTS tools (to be addressed in Chapter 2)

- To describe and characterise the viruses causing the disease (to be addressed in Chapter 2)

- To identify the virome of German peas and the spatio-temporal distribution of these viruses (to be addressed in Chapter 3 )

- To compare three different viral enrichment approaches for HTS plant viruses/viroids detection (to be addressed in Chapter 4)

- To investigate the effect of nanoviruses infection on the feeding and probing behaviours of aphids (to be addressed in Chapter 6)

- To investigate the effect of FBNSV-NSP on the feeding and probing behaviours of aphids (to be addressed in Chapter 6) 


\section{Part one: Plant virus identification}


14 | P a g e 


\section{Chapter 2: Plant disease aetiology}

\subsection{Characterisation of a novel}

nucleorhabdovirus infecting alfalfa (Medicago sativa)

Yahya Zakaria Abdou Gaafar, K. R. Richert-Pöggeler, C. Maaß, H.-Josef Vetten and H. Ziebell

This article has been published in a slightly modified version as:

Gaafar YZA, Richert-Pöggeler KR, Maaß C, 2019. Characterisation of a novel nucleorhabdovirus infecting alfalfa (Medicago sativa). Virology Journal 16, 113. doi: 10.1186/s12985-019-1147-3. 


\subsubsection{Abstract}

\subsubsection{Background}

Nucleorhabdoviruses possess bacilliform particles which contain a single-stranded negative-sense RNA genome. They replicate and mature in the nucleus of infected cells. Together with viruses of three other genera of the family Rhabdoviridae, they are known to infect plants and can be transmitted by arthropod vectors, during vegetative propagation, or by mechanical means. In 2010, an alfalfa (Medicago sativa) plant showing virus-like symptoms was collected from Stadl-Paura, Austria and sent to Julius Kühn Institute for analysis.

\subsubsection{Methods}

Electron microscopy (EM) of leaf extracts from infected plants revealed the presence of rhabdovirus-like particles and was further used for ultrastructural analyses of infected plant tissue. Partially-purified preparations of rhabdovirus nucleocapsids were used for raising an antiserum. To determine the virus genome sequence, high throughput sequencing (HTS) was performed. RT-PCR primers were designed to confirm virus infection and to be used as a diagnostic tool.

\subsubsection{Results}

EM revealed bacilliform virions resembling those of plant-infecting rhabdoviruses. HTS of ribosomal RNA-depleted total RNA extracts revealed a consensus sequence consisting of 13,875 nucleotides (nt) and containing seven open reading frames (ORFs). Homology and phylogenetic analyses suggest that this virus isolate represents a new species of the genus Nucleorhabdovirus (family Rhabdoviridae). Since the virus originated from an alfalfa plant in Austria, the name alfalfa-associated nucleorhabdovirus (AaNV) is proposed. Viroplasms (Vp) and budding virions were observed in the nuclei of infected cells by EM, thus confirming its taxonomic assignment based on sequence data.

\subsubsection{Conclusions}

In this study, we identified and characterised a new nucleorhabdovirus from alfalfa. It shared only $39.8 \%$ nucleotide sequence identity with its closest known relative, black currant-associated rhabdovirus 1 . The virus contains an additional open reading frame (accessory gene) with unknown function, located between the matrix protein and the glycoprotein genes. Serological and molecular diagnostic assays were designed for future screening of field samples. Further studies are needed to identify other natural hosts and potential vectors.

\section{Keywords}

electron microscopy; high throughput sequencing; Lucerne; rhabdovirus; alfalfaassociated nucleorhabdovirus 


\subsubsection{Background}

Alfalfa or lucerne (Medicago sativa L.), a member of the Fabaceae family, is used as perennial forage crop which is important as fodder for livestock, as green manure for soil fertility, and can be used as food and medicine for humans (Marston et al., 1943; Douglas et al., 1995; Gray \& Flatt, 1997; Peoples et al., 2001). It is grown worldwide in temperate zones. Similar to other legumes, alfalfa is susceptible to a range of pests and pathogens (Samac et al., 2016). Alfalfa can be infected by a large number of viruses such as alfalfa mosaic virus (AMV) (family: Bromoviridae) and two rhabdoviruses (alfalfa dwarf virus (ADV) and lucerne enation virus (LEV)) (Hull, 1969; Alliot \& Signoret, 1972; Bejerman et al., 2011; Bejerman et al., 2015).

Members of the Rhabdoviridae family (order Mononegavirales) infect humans, invertebrates, vertebrates and plants (Augusto Lopez et al., 1992; Longdon et al., 2010; Bejerman et al., 2011; Galinier et al., 2012). Typically, their virions are bacilliform or bulletshaped, composed of a helical nucleocapsid coated by a matrix layer and a lipid envelope while some have non-enveloped filamentous virions. The family has eighteen genera including 135 assigned species (Amarasinghe et al., 2018). Sixteen genera have a monopartite genome while two are bipartite. Their genomes are linear and consist of negative-sense, single-stranded RNA (-ssRNA) (11-16 kb in length) and can comprise up to ten or more genes. They have five canonical genes that may be overprinted, overlapped or interspersed with additional accessory genes (Fu, 2005; Walker et al., 2011; Walker et al., 2015). Viruses of the genera Cytorhabdovirus, Dichorhavirus, Nucleorhabdovirus and Varicosavirus are known to infect plants (Walker et al., 2018).

The genus Nucleorhabdovirus has currently ten assigned species. Nucleorhabdoviruses are known to be transmitted by leafhoppers (Cicadellidae), planthoppers (Delphacidae) and aphids (Aphididae) (Sylvester \& Richardson, 1992; Nault, 1997; Walker et al., 2018). Additionally, some can also be transmitted during vegetative propagation or by mechanical means. They can replicate in both plants and insect vectors (Goodin \& Min, 2012). In plant cells, they replicate in the nucleus which becomes enlarged and develops large granular nuclear inclusions. They have non-segmented genomes, and like other rhabdoviruses they have highly conserved regulatory regions separating their genes, and complementary $3^{\prime}$ leader $(\mathrm{I})$ and $5^{\prime}$ trailer $(\mathrm{t})$ sequences. The $3^{\prime} \mathrm{l}$ and $5^{\prime} \mathrm{t}$ complementary sequence has the ability to form a putative panhandle structure suggested to be involved in genome replication (Jackson et al., 2005).

With the advances in molecular techniques and bioinformatic tools, several new members of the Rhabdoviridae have been identified recently (Dilcher et al., 2015; Axén et al., 2017; Liu et al., 2018; Økland et al., 2018; Wu et al., 2018). In this study, we succeeded in sap transmission of a rhabdovirus from $M$. sativa to Nicotiana benthamiana and identified it as a hitherto undescribed nucleorhabdovirus for which we propose the tentative name alfalfa-associated nucleorhabdovirus (AaNV). 


\subsubsection{Methods}

\subsubsection{Sample source and virus isolates used}

During a survey in Stadl-Paura (Austria) in May 2010, a sample was collected by Dr. Herbert Huss from an alfalfa plant showing virus-like symptoms (symptoms were not recorded at the time) and sent to Julius Kühn Institute (JKI) for analysis. In initial attempts at virus isolation by sap transmission, the putative virus was transmitted to $N$. benthamiana seedlings as described below for further analysis and virus propagation (JKI ID 24249). For comparative studies, physostegia chlorotic mottle virus (PhCMoV; JKI ID 26372) and eggplant mottled dwarf virus (EMDV; JKI ID 29094) were maintained on $N$. benthamiana under greenhouse conditions by serial mechanical transmission.

\subsubsection{Electron microscopy}

For electron microscopy, small pieces (ca. $5 \mathrm{~mm}$ in diameter) of symptomatic leaves from $N$. benthamiana ( 5 to 7 weeks post inoculation) were directly homogenized in 2-5-fold volume of negative stain solution. This consisted of $2 \%$ ammonium molybdate, $\mathrm{pH} 6.5$, with one drop of $0.5 \%$ bovine serum albumin (BSA) added. Viral particles were adsorbed by floating a pioloform carbon-coated copper grid for 5 min on the crude sap preparation. Finally, grids were rinsed with 5 drops of $2 \%$ ammonium molybdate and dried. The preparations were used for size measurements of virions including spikes.

Immunosorbent electron microscopy (ISEM) and immunoelectron microscopy (IEM) decoration experiments targeting the viral nucleocapsid protein were done as described in (Milne, 1984; Milne \& Lesemann, 1984), using the JKI-1607 antiserum to AaNV. Fragments (ca. $2 \mathrm{~mm}$ in diameter) of a younger frizzy leaf from systemically infected $N$. benthamiana were embedded in Epon 812 after consecutive fixation of samples with $2.5 \%$ glutaraldehyde and $0.5 \%$ osmium tetroxide.

Ultrathin sections of $70 \mathrm{~nm}$ were prepared with an ultramicrotome UC7 (Leica, Germany) using a DiATOME diamond knife (Switzerland) and were placed on 75 mesh pioloform carbon-coated nickel grids. The grids were stained with $1 \%$ uranyl acetate for $30 \mathrm{~min}$ and grids were examined in a Tecnai G2 Spirit electron microscope at $80 \mathrm{kV}$. Images were taken with a $2 \mathrm{~K}$ Veleta camera. Brightness and contrast were adjusted when necessary using Adobe Photoshop CS6.

\subsubsection{Purification of rhabdovirus nucleocapsids}

Isolation of rhabdovirus nucleocapsids was performed using a modification of a method described by Roggero et al. (Roggero et al., 2000; Verbeek et al., 2013). Briefly, $100 \mathrm{~g}$ infected leaf materials of $N$. benthamiana were blended for 1 min in $500 \mathrm{ml}$ homogenisation buffer consisting of $100 \mathrm{mM}$ Tris- $\mathrm{HCl}, \mathrm{pH}$, containing $20 \mathrm{mM}$ sodium sulfite, $10 \mathrm{mM} \mathrm{Na-DIECA}$ and $5 \mathrm{mM} \mathrm{Na-EDTA}$. The homogenate was filtered through cheesecloth and centrifuged at $3000 \mathrm{rpm}$ for $10 \mathrm{~min}$ in a GSA rotor (Sorvall). The supernatant was centrifuged at $25,000 \mathrm{rpm}$ for $30 \mathrm{~min}$ in a $45 \mathrm{Ti}$ fixed-angle rotor (Beckman Coulter), and the pellets were resuspended in $180 \mathrm{ml}$ homogenisation buffer plus $2 \%\left(\mathrm{w} / \mathrm{v}\right.$ ) lauryl sulfobetaine and stirred for $1 \mathrm{~h}$ at $4{ }^{\circ} \mathrm{C}$, followed by centrifugation at 
$9000 \mathrm{rpm}$ for $10 \mathrm{~min}$ in a GSA rotor (Sorvall). The supernatant was placed onto a $20 \%$ sucrose cushion in homogenisation buffer $(3.5 \mathrm{ml} /$ tube) and ultracentrifuged at $25,000 \mathrm{rpm}$ for $2.5 \mathrm{~h}$ in a SW $28 \mathrm{Ti}$ rotor (Beckman Coulter). Then, the pellets were resuspended in $1 \mathrm{ml} 10 \mathrm{mM}$ Tris- $\mathrm{HCl}, \mathrm{pH}$, and centrifuged at 14,000 rpm in a MiniSpin centrifuge (Eppendorf). The supernatant was then placed onto preformed cesium sulfategradients $(260,405$ and $575 \mathrm{mg} / \mathrm{ml}$ [w/v], respectively) in $10 \mathrm{mM}$ Tris- $\mathrm{HCl}, \mathrm{pH} 8$, and ultracentrifuged at 35,000 rpm for $20 \mathrm{~h}$ in a SW 55 Ti rotor (Beckman Coulter). Opalescent bands were collected with a peristaltic pump, diluted to $25 \mathrm{ml}$ with $10 \mathrm{mM}$ Tris- $\mathrm{HCl}, \mathrm{pH}$ 8, and ultracentrifuged at 40,000 rpm for $3 \mathrm{~h}$ in a 70 Ti rotor (Beckman Coulter). The resulting pellet was resuspended in $5.5 \mathrm{ml} 10 \mathrm{mM}$ Tris- $\mathrm{HCl}, \mathrm{pH} 8$, and used for nucleocapsid quantification by UV spectroscopy, for EM examination and antiserum production.

\subsubsection{Antibody production and serological detection}

For production of an antiserum to AaNV (designated JKI-1607), a purified nucleocapsid preparation (approximately $250 \mu \mathrm{g} / \mathrm{ml}$ in $0.01 \mathrm{M}$ Tris- $\mathrm{HCl}, \mathrm{pH} 8.0$ ) was mixed with Freund's complete adjuvant (1:1) and injected directly into the hindleg muscles (IM) of a cross-bred rabbit. Such injections were repeated two times using Freund's incomplete adjuvant after 1 week and after 9 weeks. One week after the last injection, the rabbit was bled at weekly intervals for 1 month. Immunoglobulin G (IgG) isolation and conjugate production were performed according to (Clark \& Adams, 1977). The specificity of the AaNV IgGs was tested at a dilution of 1:1000 [v/v] in a DAS-ELISA format using extracts from EMDV-, PhCMoV- and AaNV-infected N. benthamiana (Clark \& Adams, 1977). In reciprocal DAS-ELISA experiments, antisera to EMDV (JKI-1073) and PhCMoV (JKI-2051) were tested against extracts from AaNV-inoculated plants (upper, non-inoculated leaves). DAS-ELISA was also performed to confirm the presence of AaNV in plants inoculated for the (limited) host range study. The calculation of cut-off values for each ELISA plate carried out according to the Technical Information by Bioreba (Bioreba, 2014).

\subsubsection{Whole genome sequencing}

Total RNA (totRNA) was extracted from $N$. benthamiana infected leaf material using innuPREP RNA Mini Kit (Analytik Jena AG, Jena, Germany) following the manufacturer's protocol. Ribosomal RNA (rRNA) was depleted using RiboMinus Plant kit (Invitrogen, Carlsbad, CA, USA) according the manufacturer's protocol. Random cDNA was synthesized using ProtoScript II Reverse Transcriptase (New England Biolabs, Beverly, MA, USA) and $8 \mathrm{~N}$ random primers. The second strand was synthesized with NEBNext Ultra II Non-Directional RNA Second Strand Synthesis Module kit (New England Biolabs (NEB), Beverly, MA, USA). A library was prepared using Nextera XT Library kit (Illumina) and subsequently run on a MiSeq 33 platform as pair-end reads $(2 \times 301)$.

\subsubsection{Sequencing of $5^{\prime}$ and $3^{\prime}$ ends}

To obtain the $5^{\prime}$ and $3^{\prime}$ ends of the full-length AaNV sequence, RNA ligase mediated amplification of cDNA ends (RLM-RACE) (Liu \& Gorovsky, 1993; Coutts \& Livieratos, 2003; Li et al., 2005) and RNA poly A tailing were used, respectively. 
For the $5^{\prime}$ end, cDNA was produced using a virus specific primer ( $\mathrm{HZ}-4545^{\prime}$ ACT CTT GGT ACA GCA ACT CGT 3') located 461 bases from the end. The resulting CDNA was purified using the DNA Clean \& Concentrator kit (Zymo Research, Orange, CA, USA). An adaptor (Oligo1rev 5' $\mathrm{PO}_{4}$-GAT CCA CTA GTT CTA GAG CGG C-AminoC3 cordycepin 3' adapted from (Coutts \& Livieratos, 2003)) was ligated to the cDNA using T4 RNA ligase 1 (NEB) and the ligated CDNA was purified. PCR amplification of the $5^{\prime}$ end was performed using a primer (Oligo2for 5' GCC GCT CTA GAA CTA GTG GAT C $3^{\prime}$ ) complementary to the ligated adaptor and a virus specific primer (HZ-452 5' TCC ACA AGT TGC AAG CAG GT 3') 397 bases from the genome end. A band of approximately 400 bases was obtained.

For obtaining the $3^{\prime}$ end, totRNA was poly-A tailed with the A-Plus ${ }^{\mathrm{TM}}$ Poly(A) Polymerase Tailing kit (Cellscript, Madison, WI, USA) and cDNA was synthesized using a primer (HZ-413 5' GGA CAT TGT CCG GAT GGT CT 3') binding 361 bases from the $3^{\prime}$ end of the RNA. The $3^{\prime}$ end was amplified by PCR using HZ-413 and oligo(d)T primer (5' CCT CGG GCA GTC CTT TTT TTT TTT TTT TTT T 3') (Fletcher et al., 2016).

The PCR products of both ends were cleaned using the Zymoclean Gel DNA Recovery (Zymo Research) and cloned with NEB PCR Cloning Kit (NEB). Cloning and plasmid amplification were carried out according to the manufacturer's instructions. Purification of plasmids was carried out using the NucleoSpin Plasmid EasyPure Kit (Macherey-Nagel, Düren, Germany); sequencing (ten colonies in both directions) was carried out at Macrogen (Seoul, Korea) and Eurofins Genomics (Ebersberg, Germany).

\subsubsection{Sequence analysis}

The reads produced from the MiSeq platform were analysed with Geneious software ( $v$ 11.0.4) (Biomatters Limited, Auckland, New Zealand). The raw reads were quality trimmed (error limit $=0.05$ ), size filtered $>99 \mathrm{nt}$, error corrected and normalised using BBNorm (v. 37.64) tool, followed by de novo assembly with Geneious assembler. Assembled contigs were then used to search for similar sequences by BLASTn and BLASTx using the National Centre for Biotechnology Information (NCBI) GenBank non-redundant nucleotide and protein databases, respectively. Mapping of the clean reads to the complete viral genome sequence as a reference was performed using the mapping to reference tool in Geneious. Open reading frames were identified by Find ORF tool and were used to find similar sequences and conserved domains in BLASTp.

Sequence alignments were done with clustalW and phylogenetic trees (Neighbour-Joining algorithm, 1000 bootstrap replications) were created using MEGA 7.0.26 (Larkin et al., 2007; Kumar et al., 2016). The full genome of the virus was submitted to GenBank using Sequin application (v 15.50). Importin-dependent nuclear localisation signals were predicted using cNLS Mapper (Kosugi et al., 2009) and nuclear export signals (NES) were predicted using NetNES 1.1 (La Cour et al., 2004). 


\subsubsection{Reverse transcription polymerase chain reaction (RT-PCR) for detection and confirmation}

Two primers (HZ-408 5' GCA CGA TAA AGG CTG CAT CG 3' and HZ-409 5' TTG TGC ATC CTC TGT CGG AC $3^{\prime}$ ) were designed (Geneious design new primer tool) to confirm the virus presence by RT-PCR. The primers were designed to amplify a $971 \mathrm{bp}$ fragment of the RNA-dependent RNA polymerase gene.

Extraction of totRNA was done from leaf tissues as described above, and cDNA was produced using $\mathrm{HZ}-409$ primer. The CDNA product was used for PCR using OneTaq DNA Polymerase kit (NEB) ( $35 \mathrm{cycles}$ of $30 \mathrm{~s}$ at $94{ }^{\circ} \mathrm{C}, 45 \mathrm{~s}$ at $52{ }^{\circ} \mathrm{C}, 1 \mathrm{~min}$ at $68^{\circ} \mathrm{C}$ and a final elongation step for $4 \mathrm{~min}$ at $68^{\circ} \mathrm{C}$ ). The amplified $\mathrm{PCR}$ products were subject to electrophoresis on a $1.0 \%(\mathrm{w} / \mathrm{v})$ agarose gel stained with ethidium bromide. The specificity of the designed primers was confirmed by testing EMDV-and PhCMoV-infected plants.

\subsubsection{Infectivity assays}

AaNV-infected $N$. benthamiana leaves were used to inoculate $N$. benthamiana, $M$. sativa, M. lupulina, Pisum sativum and Vicia faba mechanically. Briefly, symptomatic leaves were homogenized in Norit inoculation buffer $(50 \mathrm{mM}$ phosphate buffer, $\mathrm{pH} 7$, containing $1 \mathrm{mM}$ ethylenediaminetetraacetic acid (Na-EDTA), $20 \mathrm{mM}$ sodium diethyldithiocarbamic acid (Na-DIECA), $5 \mathrm{mM}$ thioglycolic acid, $0.75 \%$ activated charcoal and $30 \mathrm{mg}$ Celite). Using a glass spatula, the homogenate was gently rubbed onto the leaves which were then rinsed with water. The inoculated plants were kept under greenhouse conditions (at $22^{\circ} \mathrm{C}$; photoperiod of $16 \mathrm{~h}$ light [natural daylight with additional growth light Phillips IP65, $400 \mathrm{~W}$ ] and $8 \mathrm{~h}$ dark) and regularly inspected for symptoms for at least three weeks after inoculation.

\subsubsection{Results}

\subsubsection{Virus transmission and maintenance}

Upon receiving the infected alfalfa sample, the virus was mechanically inoculated onto standard indicator plants including $N$. benthamiana which were inspected for symptoms twice weekly. In $N$. benthamiana, chlorotic lesions appeared on inoculated leaves followed by systemic leaf rolling, mottling and yellowing in week three or four post inoculation. The virus was maintained continuously on $N$. benthamiana by regular mechanical passage onto young seedlings.

\subsubsection{Virus morphology and cellular localisation}

To elucidate the aetiology of the alfalfa disease, transmission electron microscopy (TEM) was performed on infected $N$. benthamiana plants following mechanical inoculation. Bacilliform-shaped virus particles were observed (Fig. 1). Using ammonium molybdate instead of uranyl acetate as negative stain was less disruptive on particle appearance. Only few mature virions displaying various degrees of disruption were detected in adsorption preparates. Preliminary measurements obtained from $n=40$ revealed virion sizes ranging from 180 to $200 \mathrm{~nm}$ in length and $85-95 \mathrm{~nm}$ in diameter. The outer surface of virions is preserved comprising the lipid bilayer carrying the spikes, likely 
glycoproteins. Virions shown in Fig. 1 are less disrupted with matrix proteins and envelope mostly intact. About $15 \%$ of the measured particles like those depicted in Fig. 1 were of shorter size (average length $167 \mathrm{~nm}$ ) and may indicate defective particles not comprising the complete viral genome.

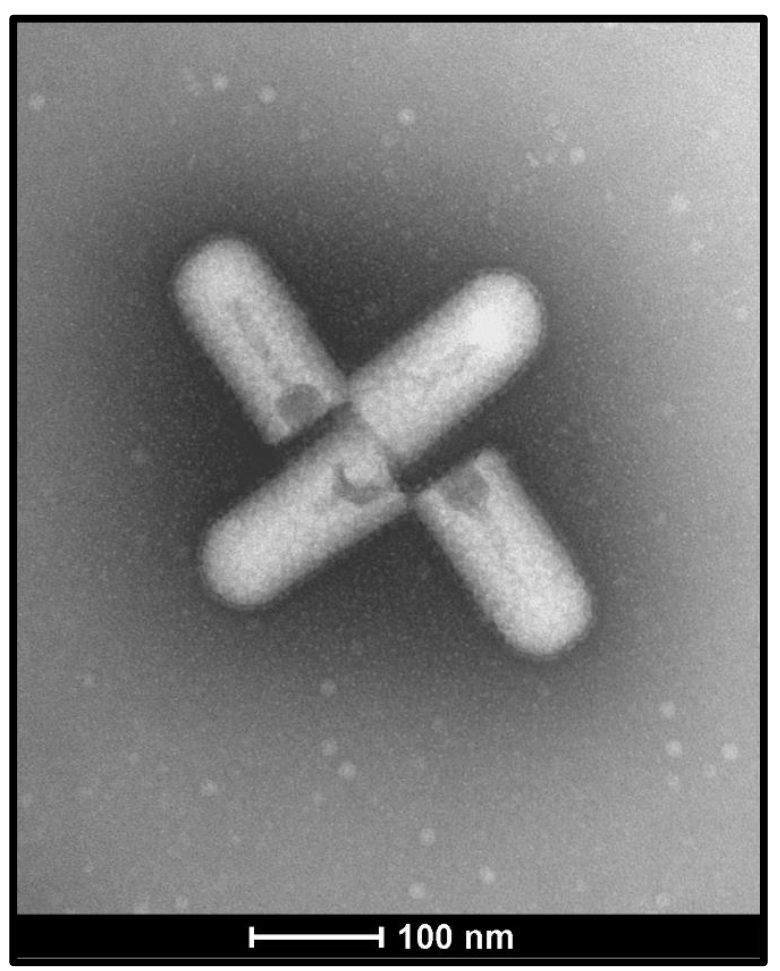

Figure 1: Electron micrograph of crude plant sap preparations of AaNV-infected $\boldsymbol{N}$. benthamiana leaves. Four shorter mature bacilliform virions with average sizes of $167 \mathrm{~nm}$ in length and $86 \mathrm{~nm}$ in diameter

When ultrathin sections of embedded symptomatic $N$. benthamiana leaf tissue were analysed, very few virus particles were found in the cytoplasm only. Figure 2a shows two virus particles in epidermal cells. The transversely cut particle seems to be complete with attachment of glycoproteins visible (lower arrow, left hand side). Figure 2a (upper arrow, right hand side) seems to show two longitudinally particles appearing blunt end to blunt end and thus looking like a larger particle. Both epidermal and mesophyl cells were infected. 


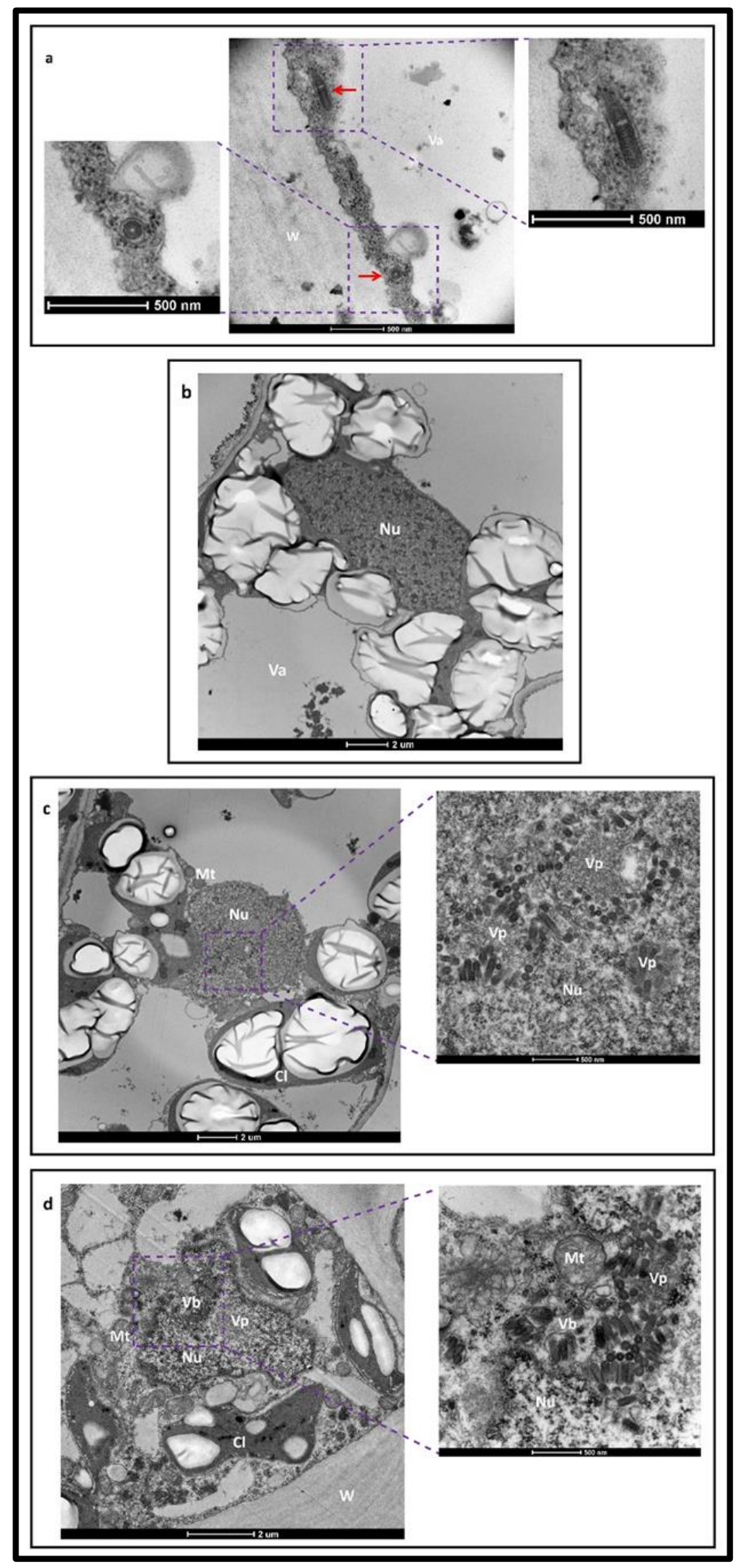

Figure 2: Electron micrographs of thin sections of AaNV-infected $\boldsymbol{N}$. benthamiana cells. (a) Arrows indicate transversely (bottom) and longitudinally (upper part) cut particles in the cytoplasm located between the cell wall (W) and the vacuole (Va) of an epidermal cell; (b) Noninfected nucleus with heterochromatin and homogenous nuclear matrix; (c) Electron-dense granular areas throughout the nucleus are thought to represent viroplasm $(V p)$ with virions placed next to it; (d) arrays of mature virions budding $(\mathrm{Vb})$ into the perinuclear space surrounded by the 
nuclear membrane. The cell wall $(\mathrm{W})$, chloroplast with starch granules $(\mathrm{Cl})$, nucleus $(\mathrm{Nu})$, mitochondrion (Mt), vacuole ( $\mathrm{Va})$, virus budding $(\mathrm{Vb})$ and viroplasm $(\mathrm{Vp})$ are indicated

In infected cells, the shape of the nucleus can change to a more condensed circular or even a distorted shape (Fig. 2c-d) compared to the ellipsoidal form present in healthy cells (Fig. 2b). In heavily infected cells, not only the nuclear compartments were affected but also chloroplasts were deformed (Fig. 2d). In the nuclei, granular areas distinct from heterochromatin were found representing putative virus replication sites known as viroplasms (Vp). Adjacent to them virus particles could be found (Fig. 2c and d). In Fig. 2c, there are few virions around the $V p$ and no virions were observed in the cytoplasm. In addition, vesicles or virus buddings $(\mathrm{Vb})$ containing one or more complete viruses were visualized around the nucleus and in the cytoplasm of infected cells (Fig. $2 \mathrm{~d}$ ). Figure $2 \mathrm{~d}$ also shows virus particles budding from the inner nuclear envelope in the perinuclear space.

\subsubsection{Sequence analysis}

A total of 1,561,664 reads were generated from the MiSeq sequencing. After quality trimming and size filtering, 1,141,662 quality-filtered reads were used for normalisation and de novo assembly. From the 23,180 assembled contigs, a contig of 13,854 nucleotides showed $66.9 \%$ identity (7\% coverage and $3 e-50$ E-value) to black currant-associated rhabdovirus 1 (BCaRV-1) (MF543022), 66.2\% (6\% coverage and 2e-45 E-value) to datura yellow vein virus (DYVV) (NC_028231) and $66.2 \%$ (9\% coverage and 5e$41 \mathrm{E}$-value) to sonchus yellow net virus (SYNV) (NC_001615) using BLASTn. Using BLASTx, the contig shared $44.9 \%$ (34\% coverage and zero E-value) identity to DYVV (YP_009176977), 43.62\% identity (35\% coverage and zero E-value) to SYNV (NP_042286) and $43.5 \%$ (34\% coverage and zero E-value) to BCaRV-1 (AUW36419). To determine the $5^{\prime}$ and $3^{\prime}$ ends, primers were designed to anneal close to the assembled contig ends. The sequences of the two ends were assembled to the contig and the full-length genome sequence was determined as 13,875 bases in length with 29,727 mapped reads, $40.6 \%$ $\mathrm{G}+\mathrm{C}$ content and mean coverage of 586X (GenBank accession number MG948563). The sequencing dataset generated in this study is available from the corresponding author upon request.

A pairwise nucleotide sequence alignment of the novel genome to selected rhabdoviruses and a phylogenetic tree was generated. ClustalW pairwise analysis showed that the AaNV sequence has $39.8 \%$ nt identity to BCaRV-1, 38.8\% to DYVV and SYNV (Supplementary Fig. S1a). Moreover, the AaNV sequence falls within the genus Nucleorhabdovirus in a clade with SYNV, BCaRV-1 and DYVV (Supplementary Fig. S1b). This clustering was supported by a neighbour joining tree of the L protein amino acid sequences of selected members of the family Rhabdoviridae (Fig. 3). 


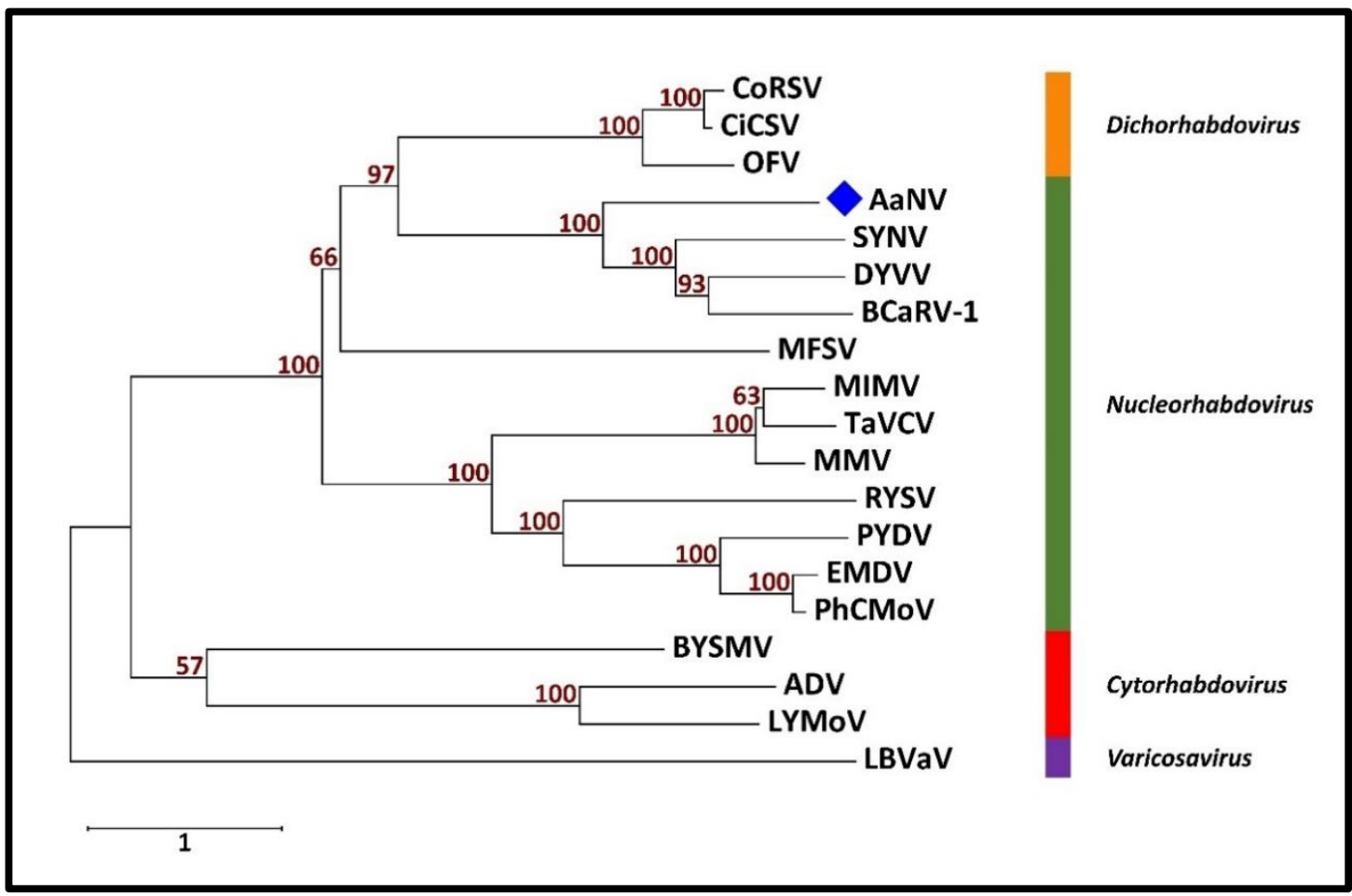

Figure 3: Unrooted neighbour-joining phylogenetic tree [Genetic distance model (Jones-TaylorThornton (JTT) model) and 1000 bootstrap replications] based on the amino acid sequence alignment of the $L$ protein of selected members of different genera of the family Rhabdoviridae. AaNV indicated by a blue solid diamond shape. The bootstrap values above $50 \%$ are indicated for each node. The names and the accession numbers of the viruses are as follow: Nucleorhabdovirus (green): alfalfa-associated nucleorhabdovirus (AaNV; QAB45076), black currant-associated rhabdovirus 1 (BCaRV-1; AUW36419), datura yellow vein virus (DYVV; YP_009176977), eggplant mottled dwarf virus (EMDV; YP_009094358), maize fine streak virus (MFSV; YP_052849), maize Iranian mosaic virus (MIMV; YP_009444713), maize mosaic virus (MMV; YP_052855), physostegia chlorotic mottle virus (PhCMoV; ARU77002), potato yellow dwarf virus (PYDV; YP_004927971), rice yellow stunt virus (RYSV; NP_620502), sonchus yellow net virus (SYNV; NP_042286) and taro vein chlorosis virus (TaVCV; YP_224083). Cytorhabdovirus (red): alfalfa dwarf virus (ADV; YP_009177021), barley yellow striate mosaic virus (BYSMV; YP_009177231) and lettuce yellow mottle virus (LYMoV; YP_002308376). Dichorhabdovirus (orange): citrus chlorotic spot virus (CiCSV; ARJ35804), coffee ringspot virus (CoRSV; YP_009507905), orchid fleck virus (OFV; YP_001294929). Varicosavirus (violet): lettuce big-vein associated virus (LBVaV; YP_002308576)

\subsubsection{The genome organisation of AaNV}

Six putative open reading frames (ORFs) were identified in the antigenomic sense based on the genome organisation described for other nucleorhabdoviruses; nucleocapsid $(N)$, phosphoprotein $(P)$, putative cell to cell movement protein $(P 3)$, matrix protein (M), glycoprotein (G) and RNA-dependent RNA polymerase (L). Highly conserved regulatory regions separating the genes were identified. At the junctions between the genes, the consensus motif is ( $3^{\prime}$ UAA AUU CUU UUU GGU UG $5^{\prime}$ ), which slightly differs between the $3^{\prime}$ leader and $\mathrm{N}$ gene, and between the $L$ gene end and the $5^{\prime}$ trailer (Fig. 4a). The presence of a seventh ORF with unknown function (U), between $M$ and $G$ was 
identified as it is flanked by the intergenic region consensus motif. Moreover, the $3^{\prime}$ leader (I) and the $5^{\prime}$ trailer ( $\mathrm{t}$ ) have complementary sequences of $43.1 \% \mathrm{nt}$ identity (Fig. $4 \mathrm{~b}$ ). Therefore, the complete genome organisation was determined as $3^{\prime} \mathrm{I}-\mathrm{N}-\mathrm{P}-\mathrm{P} 3-\mathrm{M}-\mathrm{U}-\mathrm{G}-$ L-t $5^{\prime}$ (Fig. 4c). Additionally, comparisons between the consensus sequence of the intergenic conserved sequences, the $3^{\prime}$ and the $5^{\prime}$ ends, and the genome organisation of AaNV and selective members of the Nucleorhabdovirus genus are shown in Supplementary Figure S2.

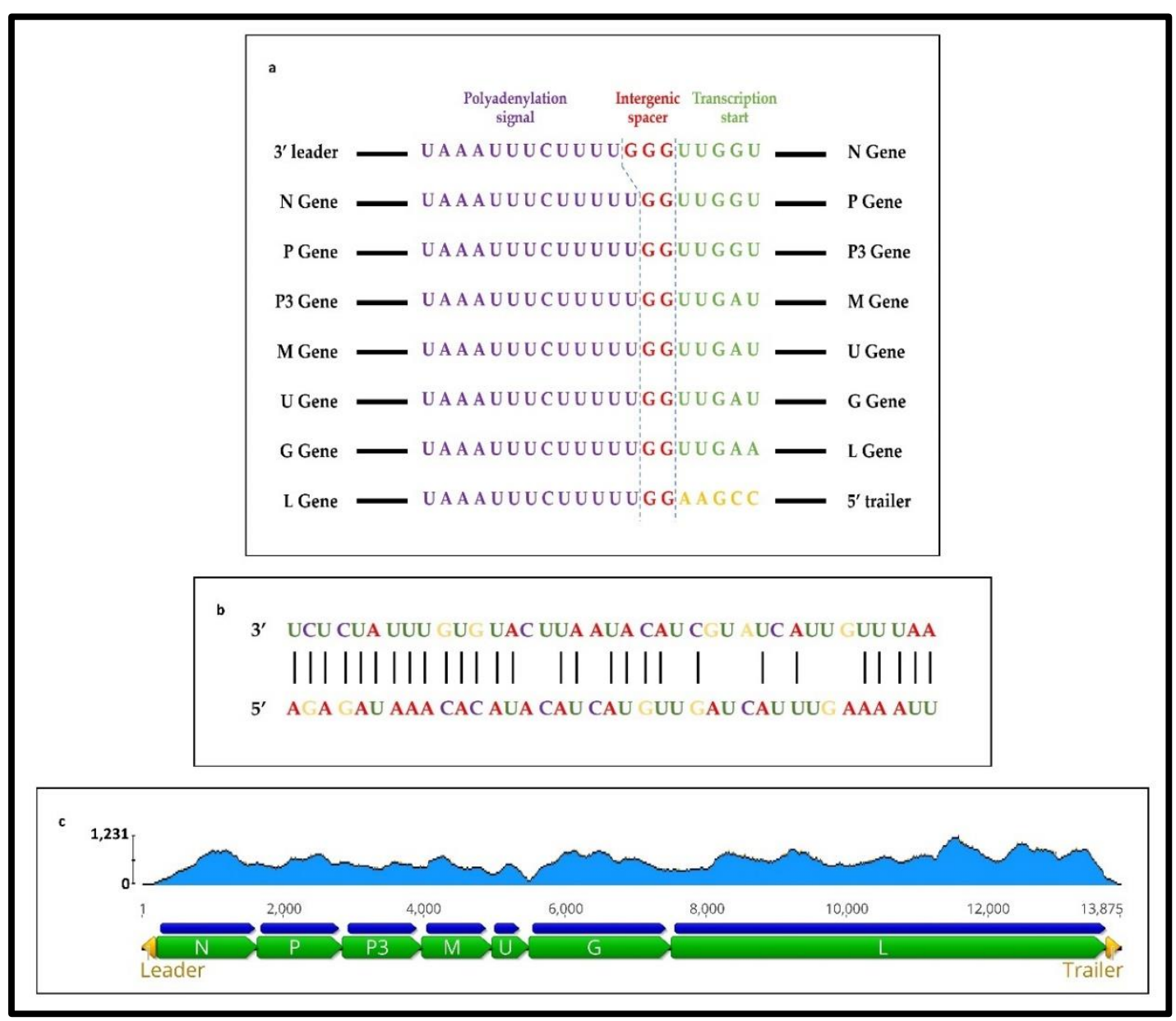

Figure 4: (a) The intergenic regions of the alfalfa-associated nucleorhabdovirus (AaNV) genome; the polyadenylation signal, the intergenic spacer and the transcription start site; (b) Alignment of ends of AaNV $3^{\prime}$ leader $(\mathrm{I})$ and $5^{\prime}$ trailer $(\mathrm{t})$ sequences (complementary nucleotides are indicated by vertical black lines); c Schematic representation of the full sequence of AaNV and its genome organisation. The open reading frames $N, P, P 3, M, U, G, L$ with their CDS are indicated as green and blue block arrows, respectively. The yellow block arrows represent the $3^{\prime}$ leader $(\mathrm{I})$ and the $5^{\prime}$ trailer $(\mathrm{t})$. The read map distribution is shown in light blue over the genome 


\subsubsection{Predicted protein features in silico}

The AaNV protein sizes range from 113 amino acid (aa) for the $U$ protein to 2038 aa for the $L$ protein with molecular masses of $12.4 \mathrm{kDa}$ and $234.8 \mathrm{kDa}$, respectively (Table 1). The $N$ and $G$ proteins have neutral isoelectric points (IEP) of 7.1 and 7.3. $U$ and $P$ are acidic proteins while $\mathrm{P} 3, \mathrm{M}$ and $\mathrm{L}$ are basic proteins (Table 1 ). Comparing the protein sequences of AaNV with those of BCaRV-1 and DYVV showed that the predicted proteins of AaNV are more closely related to those of DYVV except for the glycoprotein, which is more closely related to that of BCaRV-1 (Table 1). The aa sequences identities were between 11.5 and $35.8 \%$ compared to BCaRV-1 and between 14 and $33.7 \%$ for DYVV (Table 1). Additionally, the nuclear localisation signals (NLS, or a karyophilic domain) and the nuclear export signals (NES) of the proteins were predicted (Table 1). The highest cNLS mapper scores were for $N, P$ and $L(12.7,10$ and 10, respectively), followed by $P 3$ and $G$ with scores of 7 and 6 . The M protein had the lowest score with 4.3 while the $U$ ORF did not score any NLS. The cNLS scores predicted an exclusive nuclear localisation for N, P and $\mathrm{L}$ proteins, a partial nuclear localisation for P3 and $\mathrm{G}$ proteins, and a nuclear and cytoplasmic localisation for M protein (Table 1). Moreover, four of these proteins have a detectable NES (Table 1). 


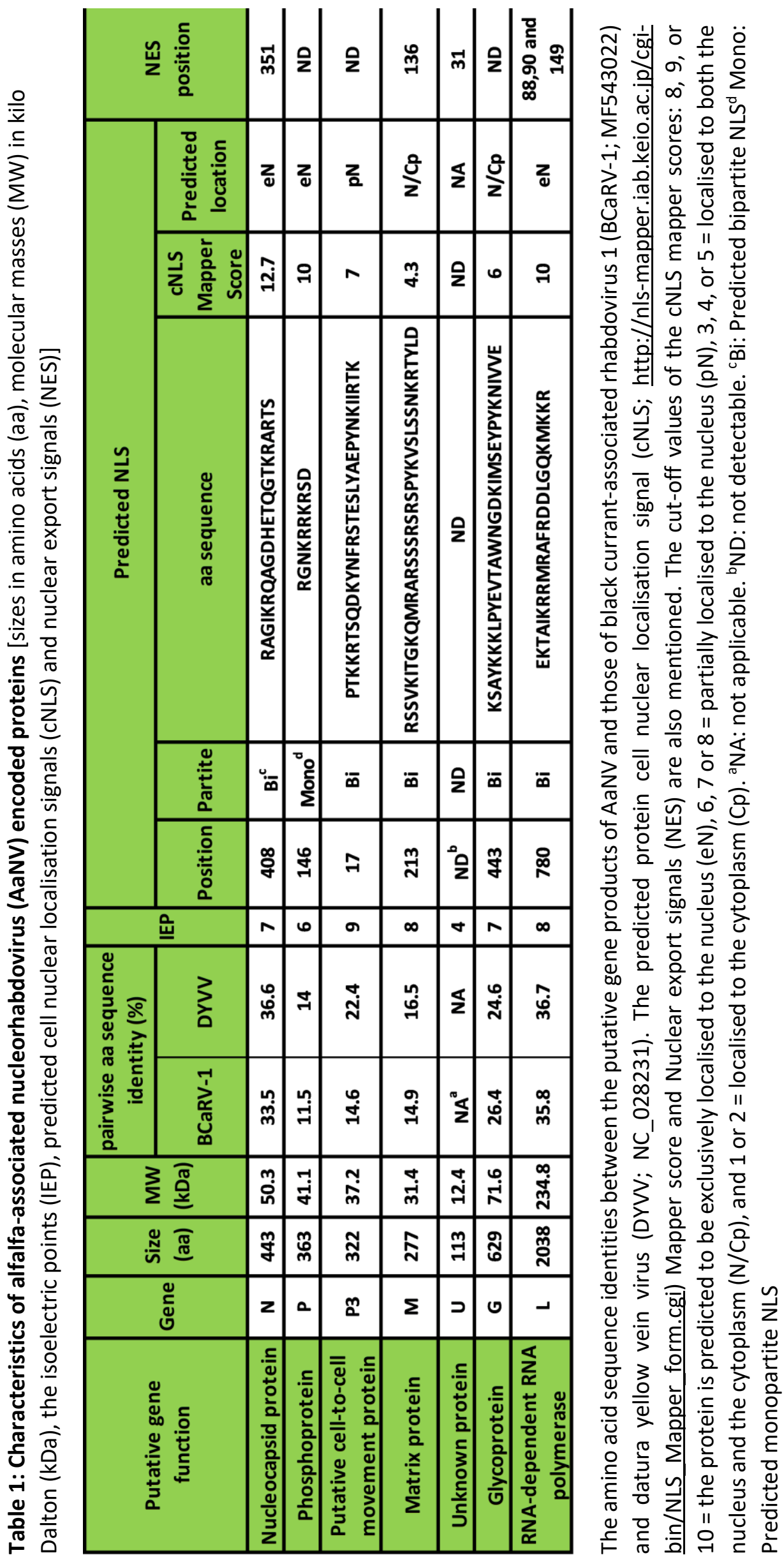




\subsubsection{Antiserum production, serological specificity and diagnostic RT-PCR}

The specificity of the AaNV antiserum was confirmed by DAS-ELISA using plant material infected with either AaNV or two other rhabdoviruses (EMDV and PhCMoV). The AaNV antibodies reacted only with plant material infected with AaNV but neither with non-inoculated nor non-infected plant material nor with plants infected with EMDV or PhCMoV (Table 1). In reciprocal tests, antisera to EMDV and PhCMoV did not react with AaNV-infected plant tissue. Diagnostic primers were designed to confirm the presence of AaNV by RT-PCR resulting in a $971 \mathrm{bp}$ amplicon. The primers were specific to the partial sequence of the L-ORF of AaNV and did not amplify other nucleorhabdoviruses tested, i.e., EMDV and PhCMoV.

The specific recognition of nucleocapsids by the AaNV antibodies were demonstrated using IEM. Only nucleocapsid structures reacted with antibodies but not complete virions, see Supplementary Fig. S3a displaying enriched but undecorated nucleocapsids from the crude sap samples after the preincubation with antiserum (immunosorbent step), and Supplementary Fig. S3b showing nucleocapsids covered with antibodies after the decoration step. With antiserum against EMDV, neither enrichment nor decoration of nucleocapsids were obtained using IEM (data not shown).

\subsubsection{Infectivity tests}

In a limited host range study, the virus was successfully transmitted to $N$. benthamiana, $P$. sativum, and $V$. faba. Mechanically inoculated $N$. benthamiana plants showed systemic infection. Systemic symptoms consisted of leaf mottling, yellowing and curling at approximately 4 weeks after inoculation (Fig. 5). However, inoculated $P$. sativum and $V$. faba showed either no symptoms or a slight leaf mottling and the infection rate was low on these hosts (only 4 out of $36 \mathrm{~V}$. faba and 1 out of $30 \mathrm{P}$. sativum plants). To confirm the infections, DAS-ELISA and RT-PCR were performed. Use of the AaNV antiserum in DAS-ELISA confirmed AaNV infections at high titres in N. benthamiana and at lower titres in P. sativum and $V$. faba and the absence of detectable virus from AaNVinoculated $M$. lupulina, $M$. sativa, T. pratense, $T$. repens and $C$. quinoa (Table 2). Infections were also confirmed by RT-PCR. It was not possible to transmit AaNV mechanically to $M$. lupulina and $M$. sativa (21 and 18 plants tested, respectively). Plants remained symptomless and all the samples tested negative in DAS-ELISA and RT-PCR. Additionally, mechanical inoculation using fresh material from AaNV-infected $V$. faba and $P$. sativum plants failed to induce infection in $V$. faba, $P$. sativum, M. lupulina and $M$. sativa. 


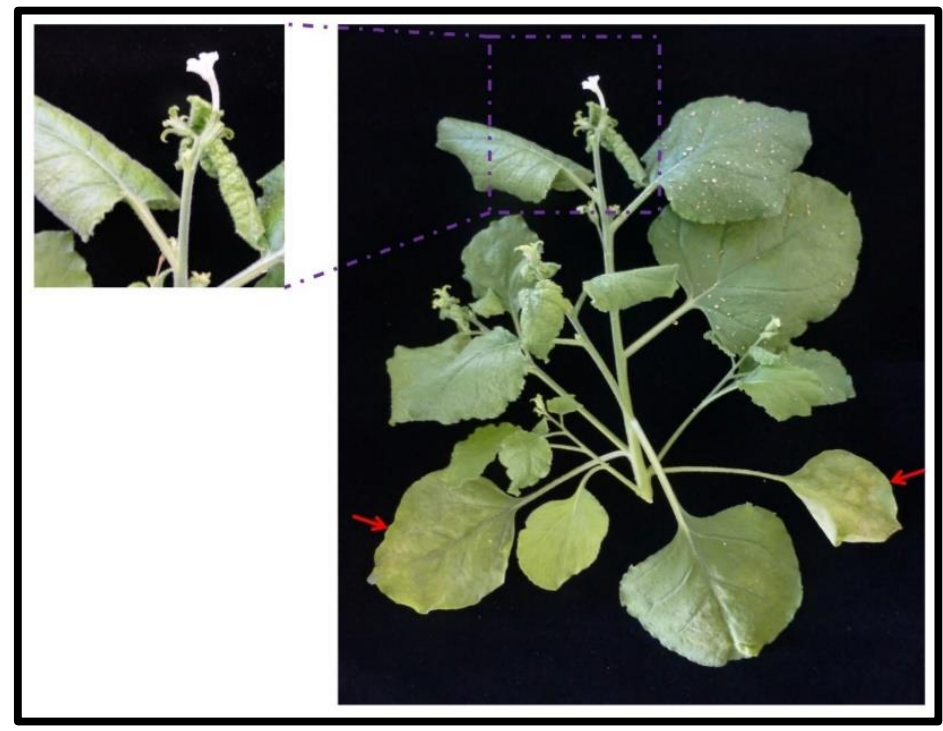

Figure 5: Photo of AaNV-infected $\mathbf{N}$. benthamiana plant. The plant shows systemic leaf rolling, mottling, yellowing and curling, and chlorotic lesions on inoculated leaves at approximately 4 weeks post inoculation. Red arrows indicating inoculated leave

Table 2: DAS-ELISA reactions of various antisera raised against different plant nucleorhabdoviruses and limited host range study

\begin{tabular}{|c|c|c|c|c|}
\hline \multirow{2}{*}{ Host species } & \multirow{2}{*}{$\begin{array}{c}\text { Inoculated } \\
\text { virus }\end{array}$} & \multicolumn{3}{|c|}{ Antisera } \\
\hline & & AaNV (JKI-1607) & EMDV (JKI-1073) & PhCMoV (JKI-2051) \\
\hline N. benthamiana & AaNV & $+++^{a}$ & - & - \\
\hline P. sativum & AaNV & + & $N T^{b}$ & NT \\
\hline V. faba & AaNV & + & NT & NT \\
\hline M. sativa & AaNV & - & NT & NT \\
\hline M. Iupulina & AaNV & - & NT & NT \\
\hline T. repens & AaNV & - & NT & NT \\
\hline T. pratense & AaNV & - & NT & NT \\
\hline C. quinoa & AaNV & - & NT & NT \\
\hline N. benthamiana & EMDV & - & +++ & - \\
\hline N. benthamiana & PhCMoV & - & - & +++ \\
\hline Buffer & & - & - & - \\
\hline N. benthamiana & $\begin{array}{c}\text { Non- } \\
\text { inoculated }\end{array}$ & - & - & - \\
\hline P. sativum & $\begin{array}{c}\text { Non- } \\
\text { inoculated }\end{array}$ & - & NT & NT \\
\hline V. faba & $\begin{array}{c}\text { Non- } \\
\text { inoculated }\end{array}$ & - & NT & NT \\
\hline M. sativa & $\begin{array}{c}\text { Non- } \\
\text { inoculated }\end{array}$ & - & NT & NT \\
\hline
\end{tabular}


${ }^{\text {a }}$ Following a substrate incubation for $1 \mathrm{~h}$, DAS-ELISA reactions were classed as follows: negative reaction (-): $\leq$ cut-off point $\left(=\mathrm{OD}_{\mathrm{A} 405} 0.025\right)$; weak reaction (+): cut-off point to 1.0 , intermediate reaction $(++): 1.0$ to 2.0 , strong reaction $(+++):>2.0) .{ }^{b} \mathrm{NT}=$ not tested

\subsubsection{Discussion}

Using EM and HTS technologies, the presence of a novel nucleorhabdovirus in alfalfa was established. The bacilliform appearance of the viral particles observed in infected $N$. benthamiana tissues is consistent with observations on previously described plant rhabdoviruses. Preliminary measurements indicated particle sizes within the range of the known nucleorhabdoviruses (130 to $300 \mathrm{~nm} \times 45$ to $100 \mathrm{~nm}$ in diameter (Goodin \& Jackson, 2002)). The virions of AaNV had an average length of $180-200 \mathrm{~nm}$ and measured 85-95 nm in diameter. The observed ultra-cellular deformations of nuclei and chloroplast in epidermis and mesophyl cells are in accordance with the distorted phenotype of systemically infected $N$. benthamiana plants showing leaf rolling, mottling and yellowing.

The species demarcation criteria for the genus Nucleorhabdovirus state that a new species should have three characteristics (Walker et al., 2018); a new species should have a minimum nucleotide divergence of $50 \%$ in cognate genes, can be clearly distinguished in serological tests or by nucleic acid hybridisation, and should occupy a different ecological niche (differences in hosts and/or vectors). The AaNV genome shares $39.8 \%$ nucleotide identity with BCaRV-1, its closest relative in the genus Nucleorhabdovirus. Moreover, all its ORFs have less than $40 \%$ amino acid sequence identity with their most closely related sequences in other rhabdoviruses. In addition, the AaNV antiserum reacted specifically with AaNV-infected plant tissue while antisera to EMDV and PhCMoV, two other nucleorhabdoviruses, failed to react with AaNV infected plant tissues in DAS-ELISA. Furthermore, the primers for RT-PCR are specific for AaNV. As for the third demarcation criterium, AaNV was originally identified in Medicago sativa, an important legume crop. However, the mode of transmission and/or potential vectors have not yet been identified. As a consequence, AaNV should be considered as a new virus species in the Nucleorhabdovirus genus.

As with all rhabdoviruses, the genome of AaNV has highly conserved regulatory regions (intergenic regions) separating its ORFs and complementary $3^{\prime}$ leader and $5^{\prime}$ trailer sequences. The intergenic regions of AaNV are closely related to those of DYVV, BCaRV-1 and SYNV (Dietzgen et al., 2015; Wu et al., 2018). The predicted features of AaNV proteins are similar to those of related nucleorhabdoviruses. The individual proteins of AaNV are similar in size to their homologs in DYVV and BCaRV-1. The predicted isoelectric point (IEP) of $\mathrm{N}$ protein of AaNV is the same as that of DYVV (Dietzgen et al., 2015). Similar to DYVV, $P 3, M$ and $L$ are basic proteins and $P$ is an acidic protein.

The only difference is the $G$ protein which is neutral in case of AaNV and acidic for DYVV. In addition to the six main nucleorhabdovirus proteins (N, P, P3, M, G and L), a new ORF $(U)$ with unknown function was identified. Its predicted protein has an acidic IEP. All 
the seven transcription units and the leader are predicted to be polyadenylated, but its functionality still needs to be proven.

Nucleorhabdoviruses are known to establish virus replication factories in the nuclei of infected plant cells (Jackson et al., 2005). All AaNV proteins except $U$, display predicted mono- or bipartite nuclear localisation sequences (NLS) suggesting their independent importation into the nucleus. The presence of both the NLSs and the leucinerich nuclear export signals (NESs) in N, M and L proteins indicates the ability of these proteins to shuttle between the nucleus and the cytoplasm through coordination of these transport signals. Although the unknown protein (U) seems to lack a NLS, the observation that it has an NES suggests its ability to be exported out of the nucleus.

AaNV was mechanically transmitted to $N$. benthaminana, P. sativum and $V$. faba. Although it did not show any noticeable or only slight mottling symptoms on $P$. sativum and $V$. faba, low infection rates were confirmed by DAS-ELISA. Interestingly, the virus could not be mechanically transmitted to $M$. sativa nor $M$. lupulina. It is not known if this is due to the serial passaging on $N$. benthamianafor propagation purposes and therefore a host adaption effect. The biological impact of the observed smaller sized particles of $167 \mathrm{~nm}$ length for mechanical transmission and host interactions awaits further investigation. As a (insect) vector has not been identified yet, it is unclear how the transmission from $M$. sativa to $M$. sativa would occur naturally or if $P$. sativum and $V$. faba crops or some weed species could act as natural alternative reservoirs for AaNV. It is also unknown if this virus still occurs naturally in alfalfa in the area it was originally found, or elsewhere in Europe. As no sequence data nor serological data are available for LEV, it is unclear whether these "historic" findings are related to AaNV.

\subsubsection{Conclusions}

In the present study, we describe a novel nucleorhabdovirus originating from infected $M$. sativa from Austria. Using HTS, we were able to determine the full-length sequence of this virus which was tentatively named AaNV. Since the sequence identity to $\mathrm{BCaRV}-1$, its closest known relative, was only $39.8 \%$, AaNV represents a new species according to the species demarcation criteria set by the International Committee on Taxonomy of Viruses (ICTV) (Walker et al., 2018). The site of virus maturation was observed by EM in the nucleus of infected cells thus confirming the phylogenetic assignment. It was possible to transfer AaNV experimentally using mechanical inoculation to $N$. benthaminana as well as other members of the Fabaceae family, i.e., P. sativum and $V$. faba. Along with ADV and LEV, this is the third rhabdovirus and the second nucleorhabdovirus known to infect $M$. sativa in nature. However, it was not possible to transfer AaNV back to alfalfa by mechanical inoculation. Thus, further research is needed to identify natural vectors of this virus as well as other alternative host plants. The serological and molecular biological assays developed may aid larger surveys addressing these questions. 


\subsubsection{Supplementary}

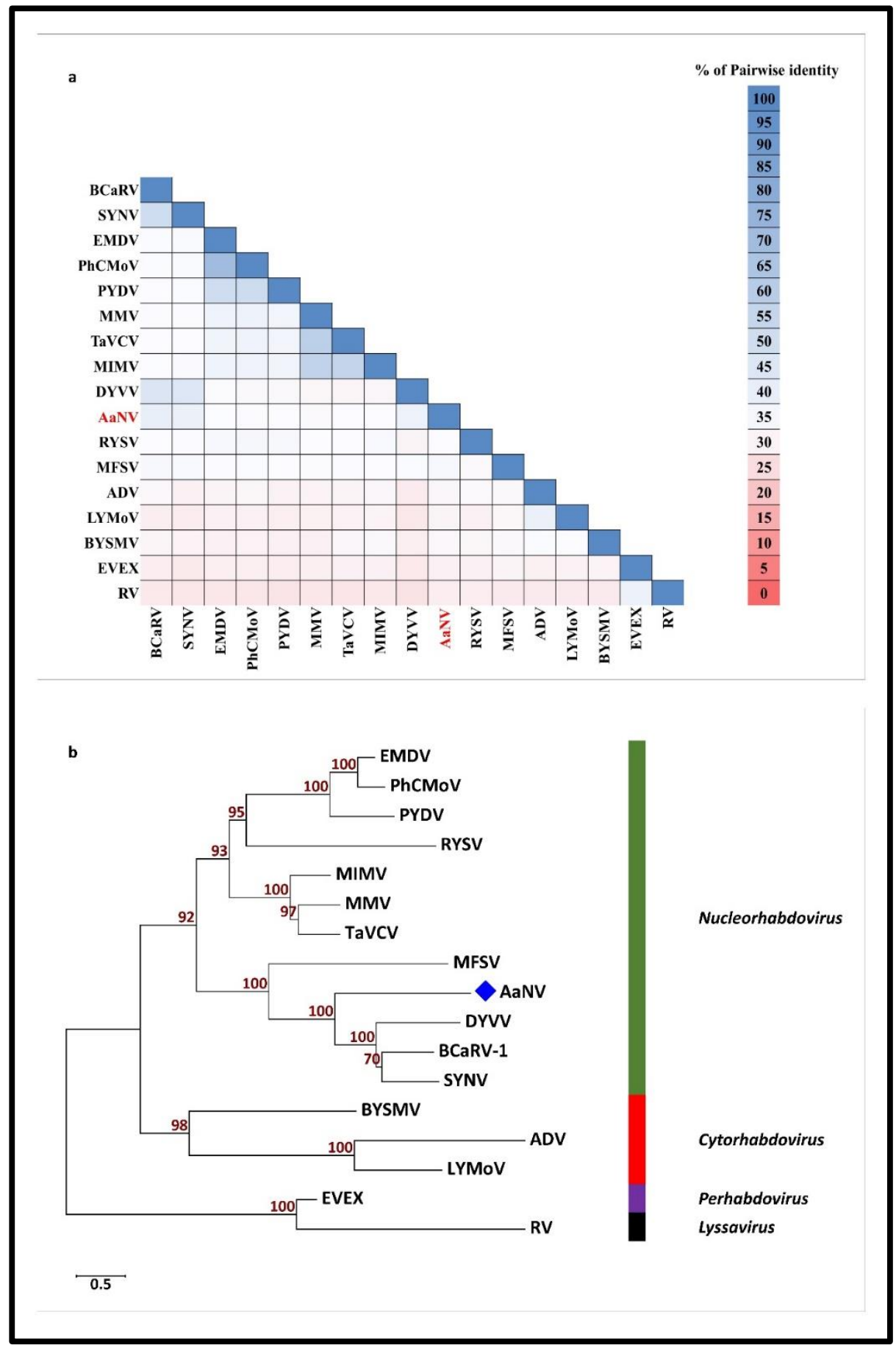

Figure S1. (a) Pairwise identity matrix of the whole genome sequences of AaNV with selected members of the family Rhabdoviridae (ClustalW 2.1); (b) Unrooted neighbour-joining phylogenetic tree [Genetic distance model (Jukes-Cantor) and 1,000 bootstrap replications] based on the nucleotide alignment of the whole genomes of AaNV and selected members of different genera of the family Rhabdoviridae. AaNV indicated by a blue solid diamond shape.

The names and the accession numbers of the viruses are as follow: Nucleorhabdovirus (green): alfalfa-associated nucleorhabdovirus (AaNV; MG948563), black currant-associated rhabdovirus 1 (BCaRV-1; MF543022), datura yellow vein virus (DYVV; NC_028231), eggplant mottled dwarf virus (EMDV; NC_025389), maize fine streak virus (MFSV; NC_005974), maize Iranian mosaic virus 
(MIMV; NC_036390), maize mosaic virus (MMV; NC_005975), physostegia chlorotic mottle virus (PhCMoV; KY859866), potato yellow dwarf virus (PYDV; NC_016136), rice yellow stunt virus (RYSV; NC_003746), sonchus yellow net virus (SYNV; NC_001615) and taro vein chlorosis virus (TaVCV; NC_006942). Cytorhabdovirus (red): alfalfa dwarf virus (ADV; NC_028237), barley yellow striate mosaic virus (BYSMV; NC_028244) and lettuce yellow mottle virus (LYMoV; NC_011532). Lyssavirus (black): rabies virus (RV; NC_001542). Perhabdovirus (violet): eel virus European X (EVEX; NC_022581).

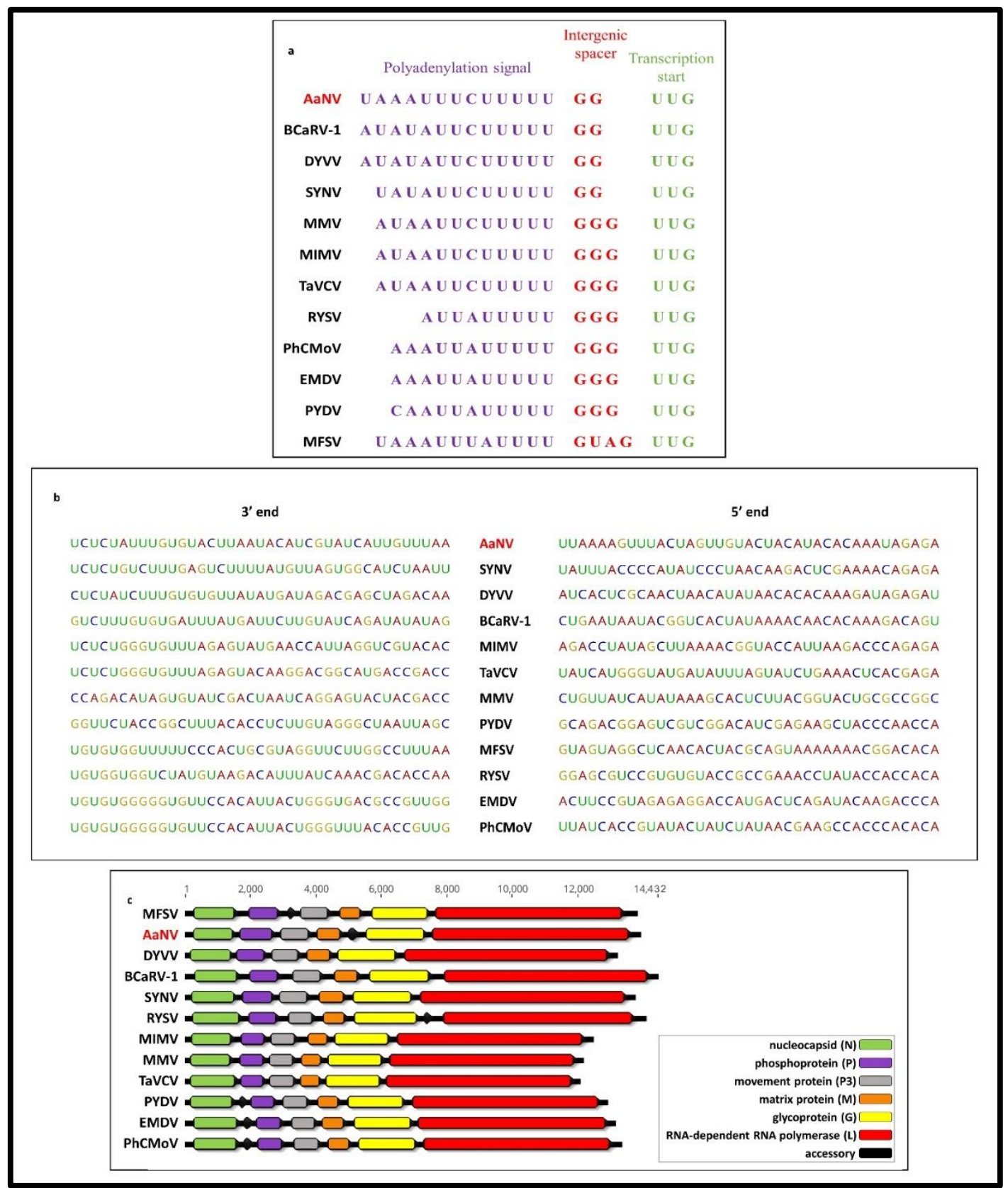

Figure S2. Comparisons between AaNV and selective members of the Nucleorhabdovirus genus. The consensus sequence of the intergenic conserved sequences (a), the $3^{\prime}$ and $5^{\prime}$ ends (b), and the genome organisation (c). The names and the accession numbers of the viruses can be found under figure S1. 


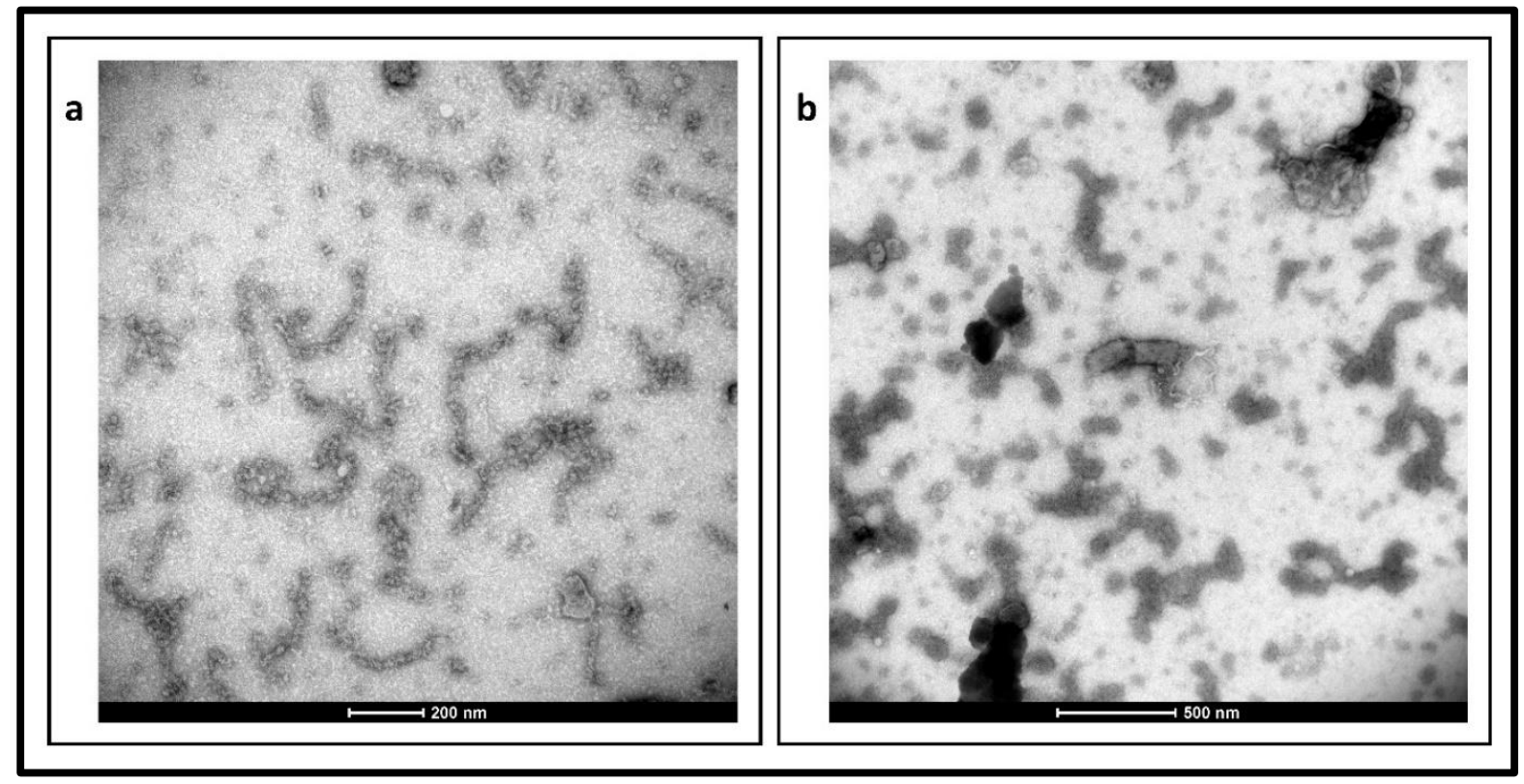

Figure S3. Electron micrograph of the JKI-1607 reacting with AaNV ribonucleoprotein (RNP). (a) Enriched nucleocapsids after immunosorbent step; (b) Enriched nucleocapsids but not virions are covered (decorated) with antibodies.

3. 


\subsection{Caraway yellows virus, a novel nepovirus from Carum carvi}

Yahya Zakaria Abdou Gaafar, K. R. Richert-Pöggeler, A. Sieg-Müller, P. Lüddecke, K. Herz, J. Hartrick, C. Maaß, R. Ulrich and H. Ziebell

This article has been published in a slightly modified version as:

Gaafar YZA, Richert-Pöggeler KR, Sieg-Müller A et al., 2019. Caraway yellows virus, a novel nepovirus from Carum carvi. Virology Journal 16, 529. doi: 10.1186/s12985-019-1181-1. 


\subsubsection{Abstract}

A novel nepovirus was identified and characterised from caraway, and tentatively named caraway yellows virus (CawYV). Tubular structures with isomeric virus particles typical for nepoviruses were observed in infected tissues by electron microscopy. The whole genome of CawYV was identified by high throughput sequencing (HTS). It consists of two segments with $8026 \mathrm{nt}$ for RNA1 and $6405 \mathrm{nt}$ for RNA2, excluding the poly(A) tails. CawYV-RNA1 shared closest nt identity to peach rosette mosaic virus (PRMV) with 63\%, while RNA2 shared $41.5 \%$ with blueberry latent spherical virus (BLSV). The amino acid sequences of the CawYV protease-polymerase (Pro-Pol) and capsid protein (CP) regions share the highest identities with those of the subgroup $C$ nepoviruses. The Pro-Pol region shared highest aa identity with PRMV (80.1\%), while the CP region shared $39.6 \%$ to soybean latent spherical virus. Phylogenetic analysis of the CawYV-Pro-Pol and -CP aa sequences provided additional evidence of their association with nepoviruses subgroup C. Based on particle morphology, genomic organization and phylogenetic analyses, we propose CawYV as a novel species within the genus Nepovirus subgroup C.

\section{Keywords}

Caraway; High throughput sequencing; Bipartite genome; Tubular structures; Nepovirus subgroup $\mathrm{C}$

\subsubsection{Main text}

Viruses from the genus Nepovirus in the subfamily Comovirinae of the Secoviridae family possess a bipartite genome consisting of two positive ssRNAs with a $5^{\prime}$ viral protein genome-linked (VPg) and a 3' poly(A) tail (Thompson et al., 2017). The RNA1 segment encodes the helicase, protease and its cofactor, replicase and the viral protein genome linked whereas the RNA2 segment encodes the movement and coat proteins (Fuchs et al., 2017; Thompson et al., 2017). Nepoviruses are the only members of the family Secoviridae known to have a single CP (Fuchs et al., 2017). Each of the two RNA segments are encapsidated separately in a non-enveloped icosahedral virion of $25-30 \mathrm{~nm}$ in diameter (Thompson et al., 2017). Nepoviruses can be transmitted non-persistently and noncirculatively by nematodes, mite and thrips (Bergeson et al., 1964; Thompson et al., 2017). Seed and pollen transmissions are well-documented (Fuchs et al., 2017; Thompson et al., 2017). In herbaceous plants, the symptoms induced are often transient with symptom recovery being a common outcome (Fuchs et al., 2017).

Caraway (Carum carvi L.) is an aromatic biennial plant in the Apiaceae family (lacobellis et al., 2005). It is native to Europe, north Africa and western Asia (Bailer et al., 2001; Eddouks et al., 2004). Caraway is used as a food flavour, fragrance additive, and for medical purposes as an antibacterial agent with antispasmodic, carminative, and appetite stimulant properties (lacobellis et al., 2005). In 2016, an organic caraway field showed 
crop losses. A caraway plant sample with systemic yellowing was sent to Julius KuehnInstitute (JKI) for analysis (Fig. 1a). The sample tested positive by DAS-ELISA using antiserum JKI 1283 developed against an uncharacterised nepovirus from carrot which is likely a strain of cherry leaf roll virus (CLRV) (unpublished data). The virus was mechanically transmitted to Nicotiana benthamiana and chlorotic local lesions were observed on inoculated leaves followed by systemic chlorosis and necrosis. Symptom recovery was not observed. The virus was provisionally named "caraway yellows virus" (CawYV).

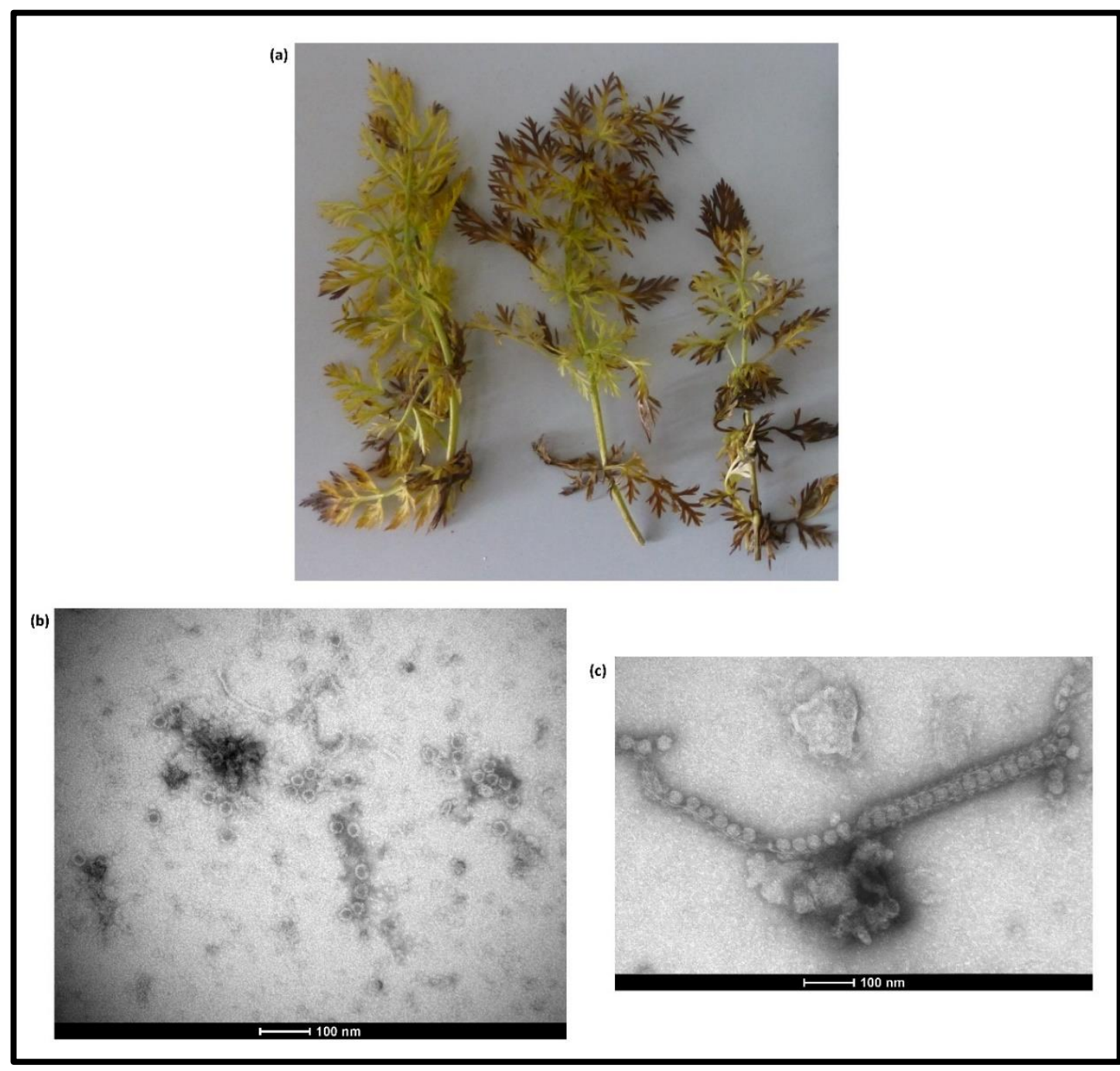

Figure 1. (a) Leaf symptoms of caraway yellows virus (CawYV) on caraway plants; (b) Electron micrograph of CawYV particles from the original infected caraway sample; (c) Electron micrograph showing tubular structure containing virus particles of CawYV in tissue homogenate of infected Nicotiana benthamiana

Electron microscopy (EM) revealed the presence of isomeric virus particles of about $30 \mathrm{~nm}$ in diameter in preparations made from the original infected caraway sample (Fig. 1b), indicating the presence of a nepovirus. Additionally, tubules containing virus-like particles in tissue homogenate of $N$. benthamiana infected with the nepovirus were also 
observed by EM (Fig. 1C). This has also been shown for other nepoviruses e.g., grapevine fanleaf virus, where the movement and the capsid proteins act as components of tubular structures (required for cell to cell movement) that traverse the cell wall with the virus particles (Laporte et al., 2003; Thompson et al., 2017).

To obtain the full genome of CawYV, double stranded RNA (dsRNA) was extracted from infected $N$. benthamiana using Double-RNA Viral dsRNA Extraction Mini Kit for Plant Tissue (iNtRON) following the manufacturer's instructions. The extracted dsRNA was sent for library preparation and high throughput sequencing (HTS) at Eurofins GATC Biotech $\mathrm{GmbH}$. The dsRNA was fragmented, strand specific CDNA was synthesized using random primers (the dsRNA was denatured at $99^{\circ} \mathrm{C}$ for $2 \mathrm{~min}$ ), followed by adapter ligation and adapter specific PCR amplification then sequencing on Illumina NovaSeq 6000 platform $(2 \times 150)$.

Using Geneious Prime (v. 2019.0.4), the raw reads $(15,468,416)$ were quality trimmed, filtered, normalized, and error corrected followed by de novo assembly using Geneious assembler (Medium sensitivity/Fast setting). 36,634 contigs of lengths between 100 and 23,141 nt were generated. A BLASTn search of the contigs against a local database for viruses and viroids downloaded from NCBI showed that two contigs of 7180 and $6341 \mathrm{nt}$ had $72 \%$ identity ( $73 \%$ coverage and zero E-value) to peach rosette mosaic virus (PRMV) and 79\% (16\% coverage and 1e-90 E-value) to blueberry latent spherical virus (BLSV), respectively. The $5^{\prime}$ ends of both RNA segments were confirmed using RNA ligasemediated amplification of cDNA ends (RLM-RACE) (Coutts \& Livieratos, 2003). The 3 ' ends of the two RNA segments were determined by using an oligo(d)T primer for CDNA synthesis followed by PCR using virus specific primers and the oligo(d)T primer. The primers used for the $5^{\prime}$ and $3^{\prime}$ ends confirmation are listed in Supplementary Table S1. The PCR products were cloned, sequenced and the resulting sequences were assembled using the map to reference tool and the original assembled contigs as references. 72,977 of the quality trimmed reads were assembled to CawYV complete genome. The assembled genome of CawYV was $8026 \mathrm{nt}$ for RNA segment 1 and $6405 \mathrm{nt}$ for RNA segment 2 (excluding poly(A) tails). The sequences were deposited in the GenBank database under accessions MK492273 and MK492274. For diagnostic purposes and to confirm the presence of CawYV in symptomatic leaf tissue, a primer pair was designed using Primer 3 tool in Geneious (HZ-636 5' TGA AGA TCC GGG AAA GGC AC 3' and HZ-637 5' ACG CTT TCC ACT CTC ACC TG $3^{\prime}$ ) (Untergasser et al., 2012). The presence of CawYV was confirmed in the infected plants by RT-PCR using OneTaq One-Step RT-PCR Kit (NEB) resulting in amplicons of $481 \mathrm{bp}$ (data not shown).

Further analyses of the CawYV sequence confirmed its identity as a nepovirus. In analogy to other nepoviruses, CawYV RNA1 contains an open reading frame (nt position 92 to 6733) encoding a polyprotein of 2213 aa in length. Pairwise comparisons of nt and aa sequences of this ORF to its homologues of the other nepoviruses were performed using ClustalW (Larkin et al., 2007). The results show that the highest similarity was shared with PRMV at $66.1 \%$ on nt and $68.1 \%$ aa levels, respectively (Table 1 ). By searching for the different nepovirus motifs using the motif searching tool in Geneious, the locations of the 
putative protease cofactor (Pro-cof), the NTP-binding helicase domain (Hel), the serine protease domain (Pro), and the RNA-dependent RNA polymerase (RdRp) core domain were found in the RNA1-encoded polyprotein (Isogai et al., 2012). The putative viral protease cofactor motif $\left(\mathrm{FX}_{27} \mathrm{WX} \mathrm{X}_{11} \mathrm{LX} 21 \mathrm{LXE}\right)$ was located at aa residues $438-502$. The typical NTP-binding helicase motif $A\left(G X_{4} G K S\right)$, motif $B(D)$, and motif $C(N)$ were found at aa $752-759,803$, and 852 , respectively. A serine protease motif was found at aa $1280-$ $1449\left(\mathrm{HX}_{40} \mathrm{EX}_{106} \mathrm{SGX}_{8} \mathrm{GX}_{5} \mathrm{GXHX} \mathrm{K}_{2} \mathrm{G}\right)$ of the CawYV RNA1 polyprotein sequence (Fig. 2a). The serine at this position is unusual for members of the Picornavirales(where cysteine is usually encoded) but was described for some members of genus Nepovirus subgroup $C$ i.e., BLSV, CLRV, PRMV and soybean latent spherical virus (SLSV). The RNA-dependent RNA polymerase (RdRp) core domain was located at aa 1774-1880 ( $D X_{4} \mathrm{DX}_{56} \mathrm{GX}_{3} \mathrm{TX}_{3} \mathrm{NX} \mathrm{B}_{33} \mathrm{GDD}$ ). Pairwise analysis of the protease-polymerase (Pro-Pol) region aa sequences showed a closest identity to PRMV Pro-Pol with $80.1 \%$ (Table 1).

Table 1. Characteristics and pairwise nucleotide (nt) and amino acid (aa) alignments of the different regions of caraway yellows virus (CawYV) and selected members of subgroup $C$ of the genus Nepovirus i.e., blueberry latent spherical virus (BLSV), blackcurrant reversion virus (BCRV), cherry leaf roll virus (CLRV), grapevine Bulgarian latent virus (GBLV), peach rosette mosaic virus (PRMV), soybean latent spherical virus (SLSV) and tomato ringspot virus (ToRSV)

\begin{tabular}{|c|c|c|c|c|c|c|c|c|c|c|}
\hline \multicolumn{3}{|c|}{ Virus } & CawYV & PRMV & BLSV & SLSV & BCRV & GBLV & ToRSV & CLRV \\
\hline \multirow{8}{*}{ nt length } & \multirow{5}{*}{ RNA 1} & Accession no. & MK492273 & NC_034214 & NC_038764 & NC_032270 & NC_003509 & NC_015492 & NC_003840 & NC_015414 \\
\hline & & Complete -poly(A) & 8026 & 8014 & 7960 & 8170 & 7711 & 7452 & 8214 & 7918 \\
\hline & & 5'UTR & 91 & 41 & 61 & 13 & 66 & 87 & 77 & 11 \\
\hline & & ORF & 6642 & 6504 & 6519 & 6588 & 6285 & 6288 & 6594 & 6339 \\
\hline & & 3'UTR & 1293 & 1469 & 1380 & 1569 & 1360 & 1077 & 1543 & 1568 \\
\hline & \multirow{3}{*}{ RNA 2} & Accession no. & МK492274 & NC_034215 & NC_038763 & NC_032271 & NC_003502 & NC_015493 & NC_003839 & NC_015415 \\
\hline & & ORF & 5022 & 4425 & 4896 & 4197 & 4881 & 4500 & 5649 & 4770 \\
\hline & & 3'UTR & 1289 & 1484 & 1393 & 1556 & 1363 & 1132 & 1547 & 1579 \\
\hline \multicolumn{11}{|c|}{ Pairwise identity \% } \\
\hline \multirow{5}{*}{ nt $\%$} & \multirow{2}{*}{ RNA 1} & Complete -poly(A) & & 63 & 60.8 & 49.6 & 38.5 & 37 & 36 & 33.8 \\
\hline & & 5'UTR & & 56.1 & 55.7 & 30.8 & 40.9 & 31.6 & 52 & 63.6 \\
\hline & \multirow{3}{*}{ RNA 2} & 5'UTR & & 37.2 & 30.9 & 34.8 & 35.9 & 36.2 & 41.3 & 63.6 \\
\hline & & ORF & & 39.9 & 38.5 & 39 & 37 & 31.8 & 40.9 & 37.7 \\
\hline & & 3'UTR & & 51 & 55.2 & 33.4 & 36.1 & 37.1 & 29.4 & 30.6 \\
\hline \multirow{7}{*}{ aa $\%$} & \multirow{7}{*}{ RNA 1} & ORF & & 68.1 & 62.5 & 48.1 & 24.5 & 22.8 & 23.1 & 22.6 \\
\hline & & $x 1$ & & 27.9 & 29.7 & 22.9 & 12.2 & 10.6 & 10.8 & 10.8 \\
\hline & & X2 Pro-cof & & 52.9 & 53.2 & 33.9 & 25.8 & 21.4 & 15.6 & 18.5 \\
\hline & & $\mathrm{Hel}$ & & 82.5 & 75.6 & 59.3 & 28 & 24.1 & 22.6 & 23.2 \\
\hline & & VPg & & 75 & 57.6 & 56.2 & 6.1 & 27.3 & 25.9 & 37.9 \\
\hline & & Pro & & 79 & 66.8 & 51.3 & 27.6 & 23.5 & 24.7 & 23.1 \\
\hline & & RdRp & & 75.5 & 68.6 & 53.9 & 33.8 & 33.6 & 33.7 & 30.9 \\
\hline
\end{tabular}




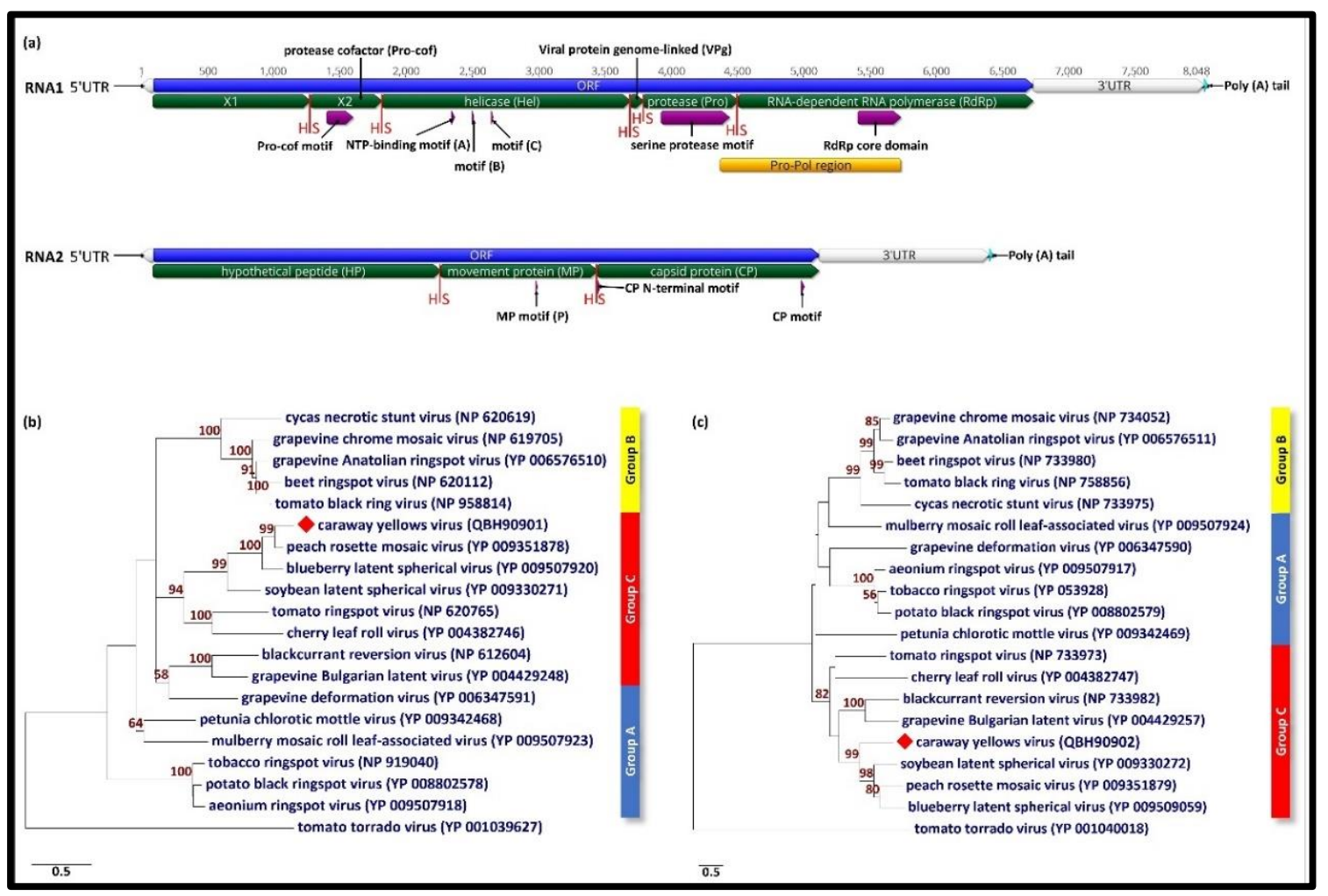

Figure 2. (a) Genome organization of CawYV-RNA1 and -RNA2. Each of RNAs 1 and 2 contain a single large open reading frame (in blue). The predicted putative peptides are shown in green, separated by the predicted cleavage site $(\mathrm{H} / \mathrm{S})(\mathrm{red})$. The RNA1 polyprotein contains X1 protein, protease cofactor (Pro-cof/X2), helicase (Hel), viral protein genome-linked (VPg), protease (Pro) and RNA-dependent RNA polymerase (RdRP). RNA2 encodes for a polyprotein with hypothetical protein (HP), movement protein (MP) and capsid protein (CP). The conserved nepovirus sequences (domains and motifs) are shown in violet. The protease-polymerase (Pro-Pol) region of CawYV-RNA1 starts with the serine (S) of the protease motif and ends with the (GDD) motif of the polymerase (shown in gold). (b) Maximum-likelihood (ML) phylogenetic trees showing the relationships between CawYV and members of the genus Nepovirus based on aa alignments of the Pro-Pol region and (c) the capsid protein (CP) region. Numbers on branches indicate the bootstrap percentages (1000 replicates, $\geq 50 \%$ are shown). Tomato torrado virus (genus Torradovirus, family Secoviridae) is an outgroup

CawYV-RNA2 contains an open reading frame (nt position 95 to 5116) encoding a polyprotein of 1673 aa in length. Pairwise comparisons of RNA2-ORF nt and aa sequences to the homologues of other nepoviruses showed the highest similarity with PRMV with $31 \% \mathrm{nt}$ and $21.3 \%$ aa identities (Table 1 ). The conserved movement protein motif (P) was found at aa position 962 (Mushegian, 1994). The CP N-terminal five amino acid residues (SGLEE) together with an alternate capsid protein (CP) motif (FXFYGWS) were located at aa positions 1119-1122 and 1631-1637 (Le Gall et al., 1995; Isogai et al., 2012). Pairwise analysis of the CP region showed that it shares highest aa identity to SLSV (39.6\%, Table 1).

Each of the CawYV polypeptides is predicted to be proteolytically cleaved into putative peptides by the virus-encoded protease. Sequence alignment of all nepovirus ORF 
aa sequences suggest a putative proteolytic cleavage sites at dipeptides $(H / S)$. This potential cleavage site was not identified before in the Secoviridae members. The conserved histidine in the substrate-binding pocket of the protease is known for members of the subgroup $C$, however the known cleavage sites are $Q / G, Q / S$ or $D / S$ (confirmed experimentally) (Thompson et al., 2017). The H/S dipeptide is also found in SLSV, BLSV and PRMV. Although the VPg motif was not confirmed in the polyprotein of RNA1, the location of the putative VPg domain could be determined by the H/S dipeptides between the NTPbinding helicase and the protease using sequence alignment (Fig. 2a). Additionally, the X1 putative protein was identified at the $\mathrm{N}$ terminus of RNA1 polyprotein by the presence of a H/S dipeptide potential cleavage site before the protease cofactor motif of X2 (Fig. 2a). The 5' untranslated regions (UTR) of the two RNAs were $91 \mathrm{nt}$ for RNA1 and $94 \mathrm{nt}$ for RNA2 and shared $61.3 \% \mathrm{nt}$ identity to each other. The 3'UTRs (1293 and $1289 \mathrm{nt}$ for RNA 1 and 2 respectively, excluding the poly(A) tail) are $98.4 \%$ identical.

A maximum likelihood tree using MEGA7 software ( $v$ 7.0.26) based on the aa alignments of the Pro-Pol and the $\mathrm{CP}$ regions were additional evidence for the relatedness of CawYV to the Nepovirus subgroup C (Fig. 2b and c) (Kumar et al., 2016).

The International Committee on Taxonomy of Viruses (ICTV) suggests the following criteria for species demarcation (Thompson et al., 2017): distinct host range; distinct vector specificity; absence of cross-protection; differences in antigenic reactions; absence of reassortment between RNA1 and RNA2; Pro-Pol region aa $<80 \%$ and CP region aa $<75 \%$ identities. Although the host range was not studied, the closest relatives of CawYV, i.e., PRMV and BLSV, are not known to infect members of the Apiaceae family. The serological cross-reactivity is well known for members of the same genus in the family Secoviridae (Thompson et al., 2017). This might explain why our antiserum raised against an uncharacterised strain of CLRV reacted with CawYV. Further investigations are necessary to test the antiserum against other nepoviruses, and attempts are currently underway to develop a CawYV-specific antiserum. When compared to the closest relative PRMV, the Pro-Pol region of CawYV is slightly above the species demarcation limit by $0.1 \%$. However, this was also observed for other nepoviruses e.g., beet ringspot virus (BRSV) and tomato black ring virus (TBRV) that share $89 \%$ aa identity at the Pro-Pol but are yet classified as distinct species (Thompson et al., 2017). However, the caraway virus-CP region is very different to other nepoviruses sharing only $39.6 \%$ aa identity with SLSV. Based on these results, we propose the assignment of CawYV as a new virus species within the subgroup $\mathrm{C}$ of the genus Nepovirus. Further studies are needed to investigate the natural mode of transmission and the biological characteristics of CawYV. 


\subsubsection{Supplementary}

Table S1: List of the primers used for caraway yellows virus 5' and 3' ends confirmation.

\begin{tabular}{|l|l|l|c|}
\hline \multirow{2}{*}{ Location } & \multicolumn{1}{c|}{ Virus Specific Primers } \\
\cline { 2 - 4 } & Name & \multicolumn{1}{c|}{ Sequence } & nt position \\
\hline \multirow{2}{*}{ RNA1-5' end } & HZ-648 & 5' GCT TGT TTA GTA GCG GCT GC 3' & 504-485 \\
\cline { 2 - 4 } & HZ-649 & 5' GCA ATC TGC AAA TAT CGT GGC T 3' & $324-303$ \\
\hline \multirow{2}{*}{ RNA2-5' end } & HZ-644 & 5' CAA TGC CCA CAA GCT TAG CG 3' & 464-445 \\
\cline { 2 - 4 } & HZ-645 & 5' ACT TTG TCA TAG CGC TCG GC 3' & 321-302 \\
\hline Adaptor & HZ-481 & 5' PO4-GAT CCA CTA GTT CTA GAG CGG C-AminoC3 3' \\
\hline Adaptor complement & HZ-482 & 5' GCC GCT CTA GAA CTA GTG GAT C 3' & NA \\
\hline \multirow{2}{*}{ RNA1-3' end } & HZ-670 & 5' GGG AGA CAT AGC ACC TCT TCT 3' & $6625-6645$ \\
\cline { 2 - 4 } & HZ-671 & 5' GAC ATG TCT CCA GAC CTA TTT TCT 3' & $6666-6689$ \\
\hline \multirow{2}{*}{ RNA2-3' end } & HZ-672 & 5' ACC CCA GCA GCT TTC ACT AC 3' & 5024-5043 \\
\cline { 2 - 4 } & HZ-673 & 5' CTA AGC CGA GAG AGG AAC GC 3' & 5073-5092 \\
\hline Poly(T)18 & HZ-012 & 5' CCT CGG GCA GTC CTT TTT TTT TTT TTT TTT T 3' & NA \\
\hline
\end{tabular}

NA: not applicable. 


\subsection{A divergent strain of melon chlorotic spot virus isolated from black medic (Medicago lupulina) in Austria}

Yahya Zakaria Abdou Gaafar, K. R. Richert-Pöggeler, A. Sieg-Müller, P. Lüddecke, K. Herz,

J. Hartrick, Y. Seide, H.-Josef Vetten and H. Ziebell

This article has been published in a slightly modified version as:

Gaafar YZA, Richert-Pöggeler KR, Sieg-Müller A et al., 2019b. A divergent strain of melon chlorotic spot virus isolated from black medic (Medicago lupulina) in Austria. Virology Journal 16, 297. doi: 10.1186/s12985-019-1195-8. 


\subsubsection{Abstract}

A tenuivirus, referred to here as JKI 29327, was isolated from a black medic (Medicago lupulina) plant collected in Austria. The virus was mechanically transmitted to Nicotiana benthamiana, M. Iupulina, M. sativa, Pisum sativum and Vicia faba. The complete genome was determined by high throughput sequencing. The genome of JKI 29327 consists of eight RNA segments closely related to those of melon chlorotic spot virus (MeCSV) isolate E11-018 from France. Since segments RNA 7 and 8 of JKI 29327 are shorter, its genome is slightly smaller (by $247 \mathrm{nts}$ ) than that of E11-018. Pairwise comparisons between the predicted virus proteins of JKI 29327 and their homologues in E11-018 showed aa identities ranging from 80.6 to $97.2 \%$. Plants infected with E11-081 gave intermediate DAS-ELISA reactions with polyclonal antibodies to JKI 29327. Since JKI 29327 and E11-018 appear to be closely related both serologically and genetically, we propose to regard JKI 29327 as the black medic strain of MeCSV. To our knowledge, JKI 29327 represents the second tenuivirus identified from a dicotyledonous plant. Serological and molecular diagnostic methods were developed for future detection.

\section{Keywords}

High throughput sequencing; Melon chlorotic spot virus; Segmented virus; Medicago sativa; Pisum sativum; Vicia faba

\subsubsection{Main text}

Members of the genus Tenuivirus, family Phenuiviridae, are plant viruses that possess non-enveloped filamentous particles and a genome consisting of four to eight single-stranded RNA segments with negative or ambisense polarity. The thin filamentous particles consist of ribonucleoprotein (RNP) complexes, measuring 3-10 nm in diameter and with lengths proportional to the sizes of the RNAs they contain. Based on the RNA sizes, the particles may appear as small, large or even branched circles (Shirako et al., 2012; Lecoq et al., 2019). Tenuivirus RNAs are neither capped at their $5^{\prime}$ end nor polyadenylated at the $3^{\prime}$ end. The nucleotide sequences of the $5^{\prime}$ and $3^{\prime}$ ends of each segment are complementary (Shirako et al., 2012). Tenuiviruses are known to be transmitted by planthoppers or by mechanical means albeit with difficulty (Shirako et al., 2012). According to the International Committee on Taxonomy of Viruses (ICTV), seven virus species are currently assigned to the genus Tenuivirus: Echinochloa hoja blanca virus (EHBV), Iranian wheat stripe virus (IWSV), Maize stripe virus (MSpV), Rice grassy stunt virus (RGSV), Rice hoja blanca virus (RHBV), Rice stripe virus (RSV) and Urochloa hoja blanca virus (UHBV). In addition, three more species have been proposed and are pending recognition by ICTV: melon chlorotic spot virus (MeCSV), Ramu stunt virus (RmSV) and wheat yellow head virus (WYHV) (Seifers et al., 2005; Mollov et al., 2016; Lecoq et al., 2019). The natural host range of tenuiviruses is typically restricted to monocots of the Poaceae family causing yield losses in important food crops such as rice (Oryza sativa L.) 
and maize (Zea mays L.) (Falk \& Tsai, 1998). The recent identification of MeCSV from melon (Cucumis melo) in France represents the first report of a tenuivirus naturally infecting a dicotyledonous plant (Lecoq et al., 2019).

In 2011, a black medic (Medicago lupulina L.) plant showing virus-like symptoms was collected in Stadl-Paura, Austria, but the symptoms were not recorded at the time. The sample was sent to Julius Kuehn Institute for analysis. Electron microscopy revealed the presence of RNP that appeared to resemble disassembled rhabdovirus particles (Iseni et al., 1998; Gaafar et al., 2019d). However, polyclonal antibodies JKI-1607 raised against alfalfa-associated nucleorhabdovirus (AaNV) (Gaafar et al., 2019d) failed to react with this virus in DAS-ELISA. The virus was transmitted mechanically as described in (Gaafar et al., 2019d) to Nicotiana benthamiana, M. Iupulina, M. sativa, Pisum sativum and Vicia faba, and was referred to as JKI 29327. Three weeks post inoculation, N. benthamiana plants showed systemic mottling, slight vein clearing and top leaf curling, whilst $M$. lupulina and $M$. sativa plants showed systemic vein clearing. $P$. sativum plants showed systemic vein clearing and severe yellowing and Vicia faba showed systemic mottling, yellowing and leaf rolling (Fig. 1). The virus particles of JKI 29327 were partially purified from infected $N$. benthamiana and used for antiserum production as described before (Gaafar et al., 2019d). The antibodies (JKI-1608) were used for DAS-ELISA analysis of sap-inoculated plants and confirmed infection of symptomatic plants.

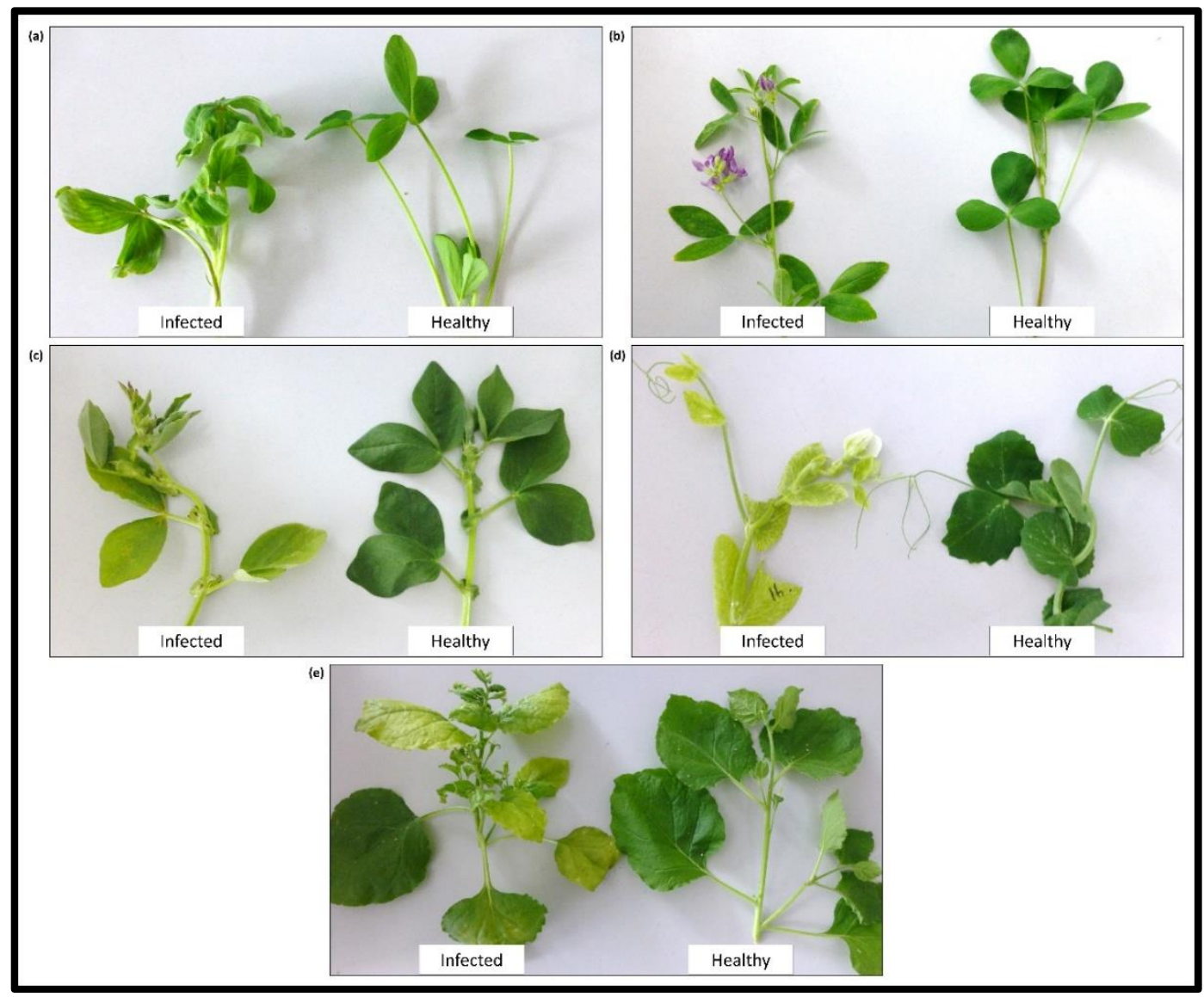

Figure 1. Plants infected with the black medic tenuivirus (JKI 29327): (a) Medicago lupulina, (b) M. sativa, (c) Vicia faba, (d) Pisum sativum and (e) Nicotiana benthamiana 
For determination of the complete genome sequence of JKI 29327, total RNA was extracted from infected $N$. benthamiana using innuPREP RNA Mini Kit (Analytik Jena AG) followed by ribosomal RNA depletion using the RiboMinus Plant kit (Invitrogen). The ribodepleted RNA was used for high throughput sequencing (HTS) on a MiSeq (v3) platform $(2 \times 301)$ as described before (Gaafar et al., 2019d). A total of 2,056,956 reads were obtained. The raw reads were quality trimmed, and size filtered using Geneious Prime ( $v$. 2019.0.3) (Biomatters Limited). The reads were then de novo assembled using Geneious assembler. A total of 53,651 contigs were generated and used for Blastn and Blastx search using virus/viroid databases on NCBI. Fifty-eight contigs shared nucleotide (nt) sequence identities (from 73.5 to $90.6 \%$ ) and amino acid (aa) sequence identities from 63.8 to $97.2 \%$ to MeCSV. No other virus sequences were detected. The reference sequences of MeCSV (NC_040448 to NC_040455) were used to map the black medic tenuivirus sequences. The complete genome sequence of JKI 29327 (containing eight segments (Fig. 2a)) was assembled (19,805 nt; accession nos. MK450511 to MK450518) but segment RNA7 and RNA8 were $94 \mathrm{nt}$ and $177 \mathrm{nt}$ shorter than the genome of the isolate E11-018 of MeCSV. Analysis of each segment showed the presence of conserved nt sequences which can also be observed in other tenuiviruses (ACA CAA AGU $C$ at the $5^{\prime}$ end with its complementary sequence UGU GUU UCA G at the $3^{\prime}$ end). Eight primers pairs were designed using Primer 3 (2.3.7) tool in Geneious (Table 1 ) to confirm the physical presence of all eight viral segments using RT-PCR (OneTaq One-Step RT-PCR Kit; NEB) (Untergasser et al., 2012) on fresh RNA extracts from $N$. benthamiana. The amplicons were gel-purified using Zymoclean Gel DNA Recovery Kit (Zymo Research) and Sanger sequenced; sequence analyses of these amplicons showed that they were $100 \%$ identical to the corresponding segment sequences obtained by the HTS analysis and thus confirmed the presence of each individual viral segment. 


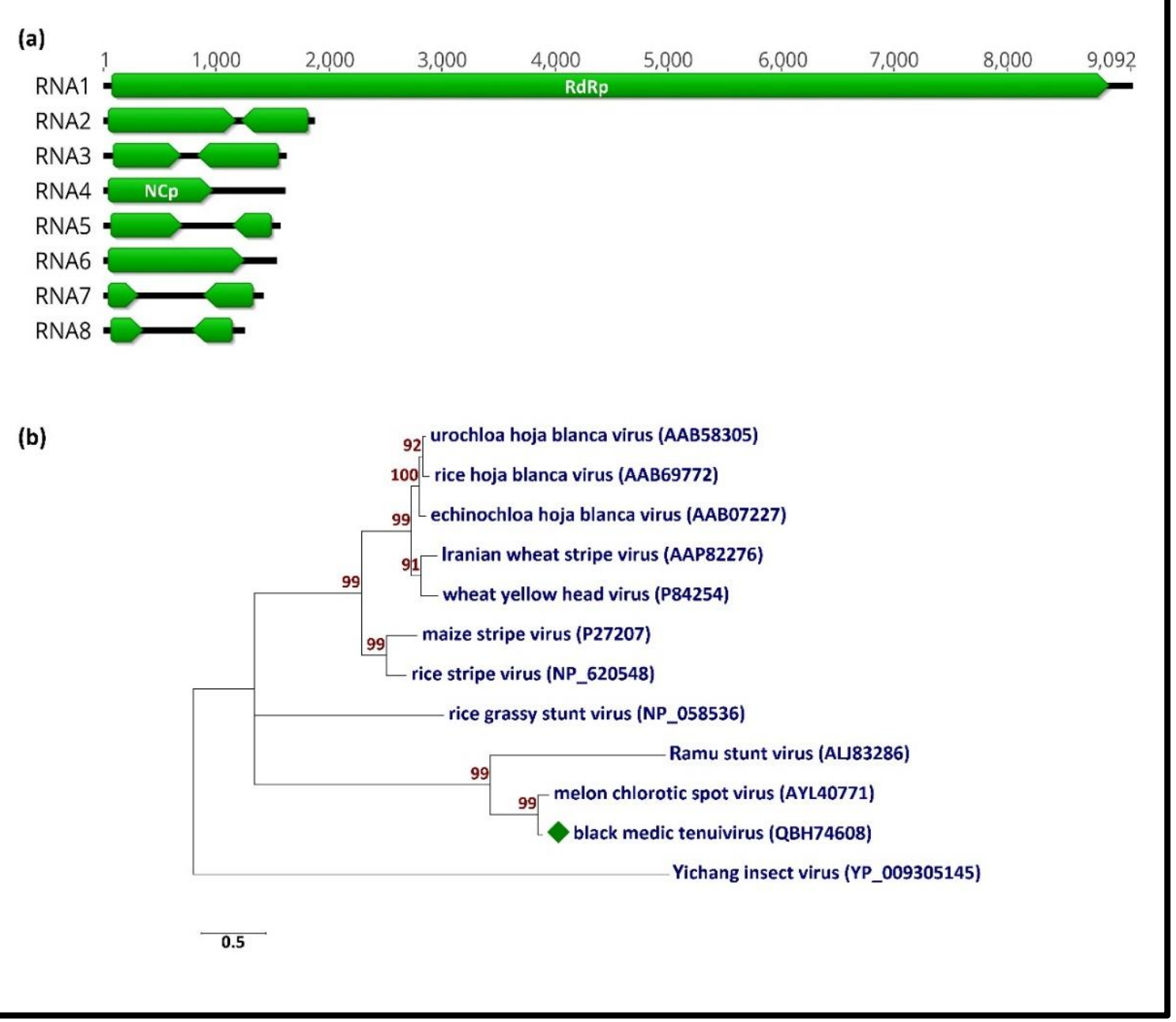

Figure 2. (a) Graphical representation of the genome of the black medic tenuivirus isolate JKI 29327. (b) Maximum-likelihood (ML) phylogenetic tree (using Jones-Taylor-Thornton (JTT) model) based on the amino acid sequence alignments of the nucleocapsid proteins (NCp) of JKI 29327 and members of the Tenuivirus genus. The GenBank accession nos. are in brackets. Yichang insect virus (genus Goukovirus) was used as an outgroup sequence. Numbers on branches indicate the bootstrap percentages ( 1000 replicates, only values $\geq 50 \%$ are shown) and the scale bar represents a genetic distance of 0.5 
ㅁ.

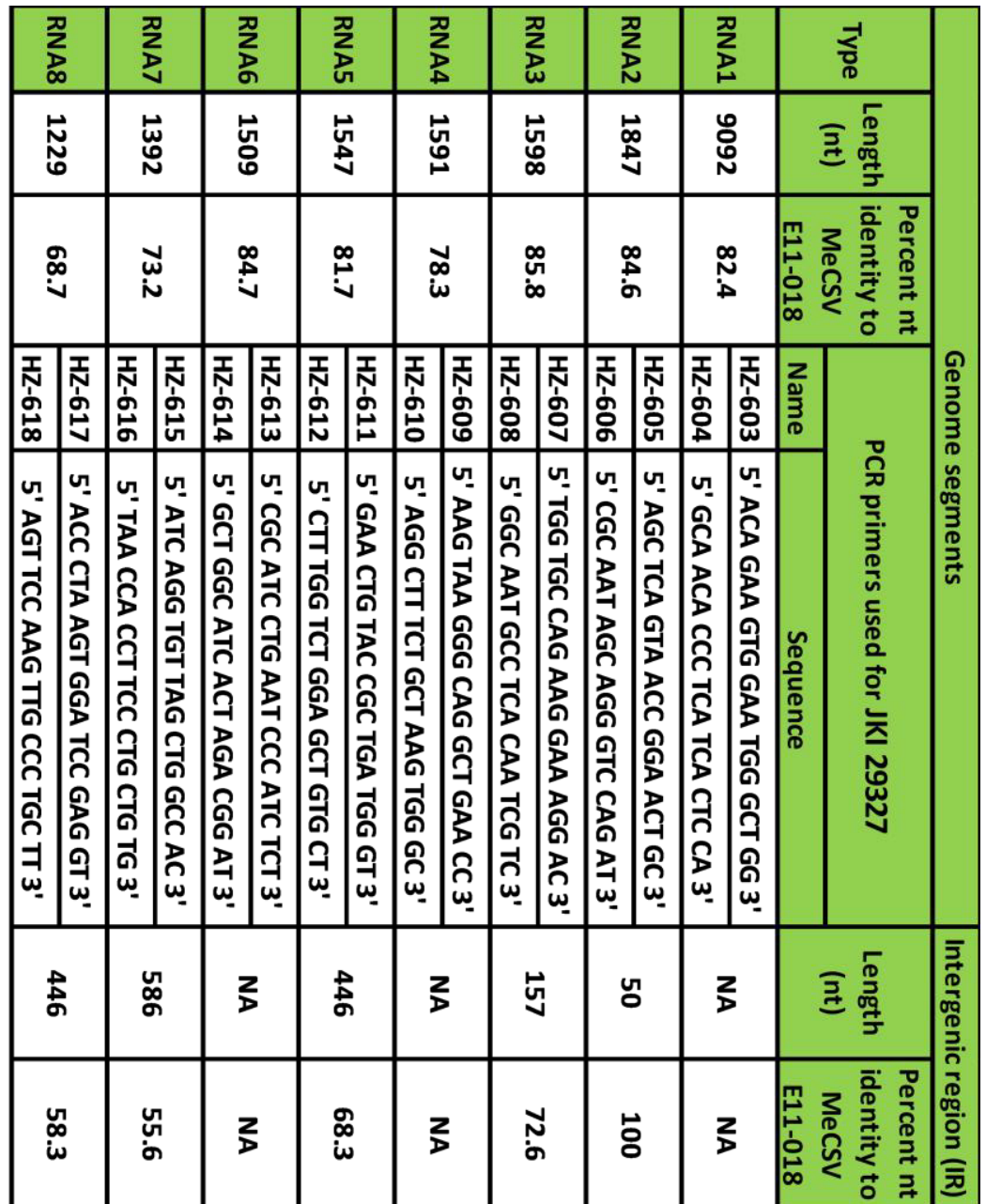

它!

ㅎํㅇำ

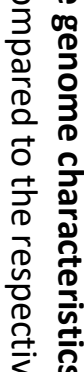

๙

웅 욱

을 옹

응

는 흥

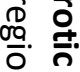

ज. ํㅡㅇ

弆

or

品

ำ

오

$3 \overline{0}$

กำ

罂

음 츠

눔

吕 크

응

흔

은

꾼

\begin{tabular}{|c|c|c|c|c|c|c|c|c|c|c|c|c|c|}
\hline 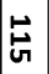 & $\stackrel{0}{\square}$ & $\stackrel{\vec{\omega}}{\vec{\omega}}$ & $\stackrel{\infty}{\triangleright}$ & $\stackrel{े}{\circ}$ & $\overrightarrow{5}$ & 祫 & 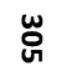 & 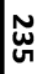 & $\bullet$ & & $\underset{\omega}{w}$ & 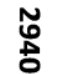 & 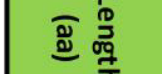 \\
\hline & 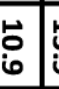 & $\begin{array}{c}\vec{v} \\
\text {. }\end{array}$ & : & $\begin{array}{l}\vec{v} \\
\dot{v}\end{array}$ & $\vec{\omega}$ & $\underset{\sim}{\sim}$ & $\underset{\omega}{w}$ & N & $\stackrel{\sim}{\sim}_{\text {N }}$ & $\underset{w}{\vec{v}}$ & $\mid \begin{array}{c}\dot{\omega} \\
\dot{\sigma}\end{array}$ & $\underset{\stackrel{\omega}{0}}{\omega}$ & 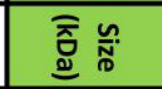 \\
\hline & ְִ & $\begin{array}{l}\infty \\
0 \\
\sigma\end{array}$ & 总 & $\stackrel{\mathscr{R}}{\stackrel{0}{*}}$ & |ढ़ & $\underset{\omega}{\stackrel{0}{\omega}}$ & $\stackrel{\substack{\infty \\
i}}{i}$ & $\begin{array}{l}\infty \\
6 \\
\infty\end{array}$ & & 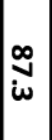 & $\stackrel{\infty}{*}$ & $\stackrel{\stackrel{2}{i ்}}{i}$ & 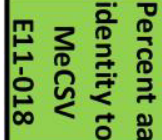 \\
\hline
\end{tabular}

궁

ปั

ร ข

可 吕

号

윽 중

उำ

D

ज

员

웅

程

of

录

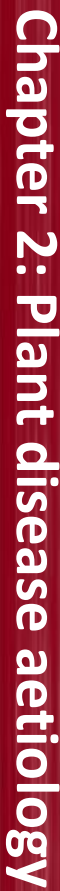


To predict the open reading frames' (ORF) functions, the translation of each ORF was used to search for conserved domains on NCBI's conserved domain database (CDD v 3.16) (Marchler-Bauer et al., 2017). Only two ORFs matched with entries in the database, i.e., RNA1-ORF1 with Bunyavirus RNA-dependent RNA polymerase (accession no.: cl20265) and RNA4-ORF1 with Tenuivirus/Phlebovirus nucleocapsid protein (accession no.: cl05345) (Table 1). Pairwise alignments for the different regions of each segment of JKI 29327 were performed with their homologous sequences of MeCSV using CLUSTALW (Table 1) (Larkin et al., 2007). The genome components of JKI 29327 shared nt identities ranging from 68.7 to $85.8 \%$ with those of the MeCSV isolate E11-0188 (Table 1). The proteins potentially encoded by JKI 29327 and E11-018 shared aa sequence identities ranging from 80.6 to $97.2 \%$ (Table 1). A maximum-likelihood (ML) phylogenetic tree was generated using MEGA7 (7.0.26) (Jones-Taylor-Thornton (JTT) model) (Kumar et al., 2016) for comparing the aa sequence of nucleocapsid proteins of JKI 29327 and other tenuiviruses. This showed a tight clustering of JKI 29327 with the MeCSV nucleoprotein (Fig. 2b). Additionally, RNA segments 7 and 8 respectively have shorter intergenic regions (IR) (586 and $446 \mathrm{nt}$ ) compared with those ( 680 and $623 \mathrm{nt}$ ) on the homologous RNAs of E11-018. The nt sequence identities between these IR regions of RNA7 and RNA8 are 55.6 and $58.3 \%$, respectively. The results indicate that JKI 29327 is closely related to but distinct from the MeCSV isolate E11-018.

For additional confirmation, purified RNP preparations of JKI 29327 were again examined by electron microscopy and shown to contain tenuivirus-like circular filamentous particles representing the individual genome segments (Fig. 3).

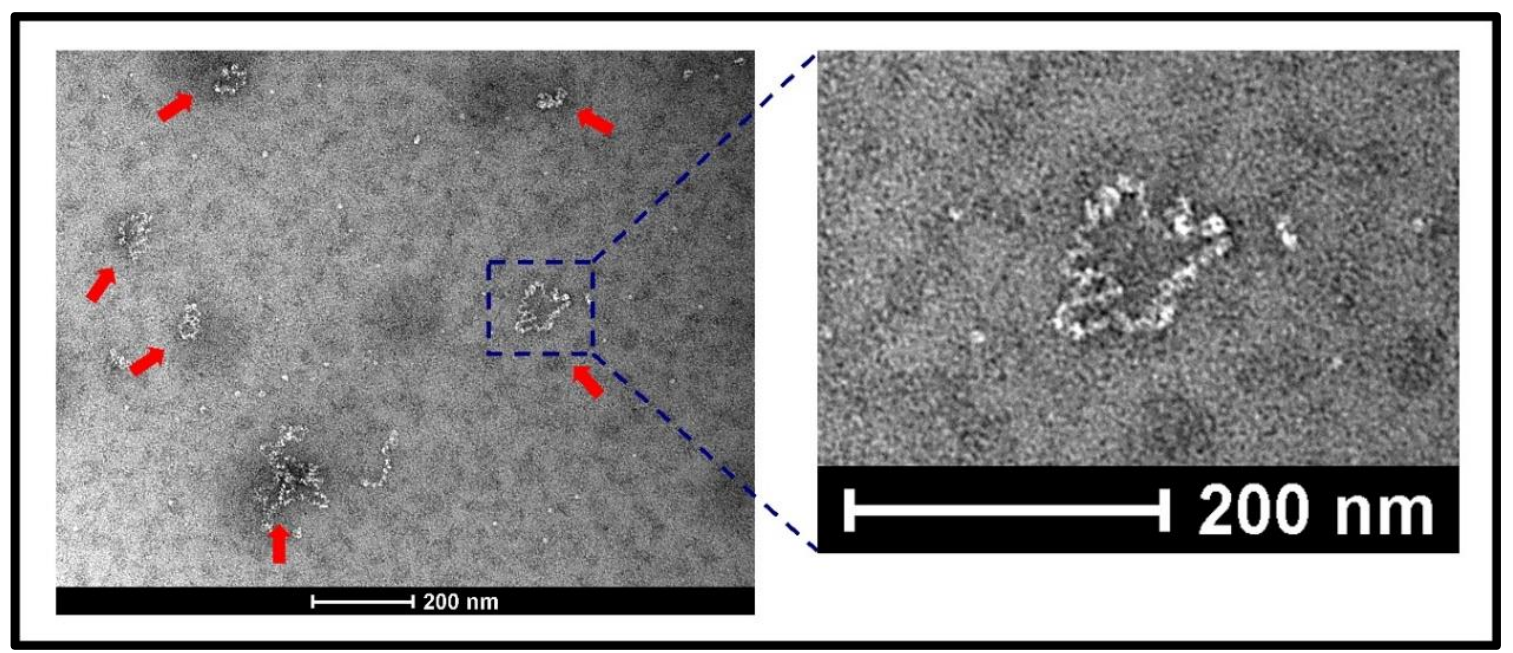

Figure 3. (a) Electron micrograph of a purified ribonucleoprotein preparation showing tenuiviruslike circular filamentous ribonucleoproteins (RNP) of different sizes (red arrows)

To assess the serological relationship between JKI 29327 and E11-018, N. benthamiana and Physalis floridana leaves infected with the MeCSV isolate E11-018 (kindly provided by Dr. C. Desbiez) were tested in DAS-ELISA using the JKI 1608 antibodies to JKI 29327. The latter gave strong ( $A_{405} \mathrm{~nm}$ values: $>2.0$ ) reactions with JKI 29327 (in four 
different plant spp.) and intermediate reactions ( $A_{405} \mathrm{~nm}: 1.0$ to 2.0$)$ with E11-018, indicating that the serological relationship between these two isolates is close. Additionally, JKI 29327 was mechanically inoculated to melon cv. Védrantais (kindly provided by Dr. C. Desbiez). The plants showed chlorotic spots only on inoculated leaves and tested positive in DAS-ELISA with the JKI 1608 antibodies. Whilst JKI 29327 could be detected in inoculated leaves, no systemic infection was observed (data not shown).

The species demarcation criteria of ICTV for the genus Tenuivirus suggest that a new species should be considered when (i) the aa sequence identities between any corresponding gene products is below 85\%; (ii) the nt sequence identities between corresponding IRs is below $60 \%$; (iii) there are different sizes and/or numbers of genomic components; (iv) there are differences in host range; ( $v$ ) the vectors are different (Shirako et al., 2012). For certain tenuiviruses, it has been difficult to decide whether they belong to the same or different species because all the five criteria are not always met (Shirako et al., 2012). For example, RHBV, EHBV and UHBV have different vectors, different hosts, different sizes and numbers of RNA segments and the nt sequence identity of their IR is $<60 \%$. Yet, the four protein homologs on their RNA3 and RNA4 are $90 \%$ identical in aa sequences.

The black medic tenuivirus isolate JKI 29327 fulfils three out of these five criteria. Firstly, its ORF2 of RNA7 shares $80.6 \%$ aa identity with its homologue in the E11-081 genome. Secondly, the IRs of both RNA7 and RNA8 share $<60 \%$ nt identities with those of E11-081. Thirdly, the overall genome size of JKI 29327 is 247 nt shorter than that of E11081. Based on these three criteria, the black medic virus should be considered a new species. However, although the host range was not studied in full detail, both JKI 29327 and E11-081 infected members of the Fabaceae, the Cucurbitaceae and the Solanaceae families under experimental conditions. Moreover, these two isolates appear to be serologically closely related when tested with the JKI 1608 antibodies. Small differences in size, particularly in the intergenic regions, are common and can be observed between isolates of RSV (Wei et al., 2009; Lu et al., 2018). Also, segment RNA 7 of MeCSV E11-018 was shown to present size heterogeneity due to indels in the intergenic region (Lecoq et al., 2019). Furthermore, only one protein out of 13 was below the $85 \%$ identity threshold. Therefore, we propose that the black medic isolate from Austria is a strain of MeCSV and is referred to accordingly as black medic strain of MeCSV. Further studies are required to identify possible natural hosts and insects that may act as vectors of both JKI 29327 and E11-081. Moreover, there is a need to compare the experimental and natural host ranges of the two MeCSV strains. The antiserum obtained in this study will help to monitor prevalence and geographic distribution of MeCSV as well as its agronomic impact on crop plants (e.g., melons, legumes). Furthermore, it is important to study the function of the virus proteins that have been predicted in silico. 


\subsection{Complete genome sequence of highly divergent carrot torradovirus 1 strain from Apium graveolens}

Yahya Zakaria Abdou Gaafar and H. Ziebell

This article has been published in a slightly modified version as:

Gaafar YZA, Ziebell H, 2019. Complete genome sequence of a highly divergent carrot torradovirus 1 strain from Apium graveolens. Archives of virology. doi: 10.1007/s00705019-04272-3. 


\subsubsection{Abstract}

A new virus was identified in a celery plant showing chlorotic rings, mosaic and strong yellowing symptoms, and its complete genome sequence was determined. The genomic organization of this novel virus is analogous to that of known members of the genus Torradovirus, consisting of two single-stranded RNAs of 6,823 (RNA1) and 4,263 nucleotides (RNA2), excluding the poly(A) tails. BLAST searches against the nucleotide and protein databases showed that this virus is closely related to but different from carrot torradovirus 1 (CaTV1). Comparisons between the two viruses demonstrated relatively low levels of nucleotide and amino acid similarity in different parts of their genomes, as well as considerable differences in the sizes of their two genomic RNAs. However, the protease-polymerase (Pro-Pol) and capsid protein (CP) regions of this virus share $>80 \%$ amino acid identity with the corresponding regions of CaTV1. Therefore, based on the current ICTV species demarcation criteria for the family Secoviridae, the virus from celery is a divergent strain of CaTV1, named "CaTV1-celery". Nevertheless, differences between CaTV1 and CaTV1-celery in genome size, as well as in biological and epidemiological features, may warrant their separation into two distinct species in the future.

\subsubsection{Main text}

A celery plant (Apium graveolens variant graveolens) was collected in August 2017 in the state of Hesse in Germany. The sample displayed mosaic symptoms with chlorotic rings and strong yellowing. To identify the possible cause of the disease, leaf material was examined by electron microscopy, but no virus particles were observed. However, mechanical inoculation of Nicotiana benthamiana, $N$. clevelandii, $N$. occidentalis-P1 and Coriandrum sativum with the sap of the celery plant resulted in systemic infections three weeks after inoculation, with symptoms consisting of chlorosis and necrosis. No virus particles could be observed in samples from symptomatic test plants. Attempts to transmit the pathogen mechanically to the original host species $A$. graveolens or to Ammi majus, Anethum graveolens, Daucus carota and Petroselinum crispum were not successful.

To characterise the genome of the suspected virus, total RNA was extracted from symptomatic N. benthamiana leaves using an innuPREP RNA Mini Kit (Analytik Jena AG). The ribosomal RNAs were depleted using a RiboMinus Plant Kit (Invitrogen) and the ribodepleted RNA was used for library preparation using a Nextera XT Library Kit (Illumina). The library was subjected to high-throughput sequencing (HTS) on a MiSeq v3 platform (2x301). The reads were quality trimmed and filtered using Geneious software (version 11.1.3) (Biomatters Limited). The high-quality reads were assembled using the Geneious de novo assembly tool. In BLASTn searches, two assembled contigs of 6,727 and 4,106 nt shared $71.7 \%$ and $70.5 \%$ identical nucleotides with the two genomic RNAs of carrot torradovirus 1 (CaTV1) (NC_025479 and NC_025480). 
The $5^{\prime}$ ends of both RNA segments were confirmed using RNA-ligase-mediated amplification of cDNA ends (RLM-RACE) (Coutts \& Livieratos, 2003). The $3^{\prime}$ ends of the two RNA segments were determined via RT-PCR using a virus-specific primer and an oligo(d)T primer. The PCR products were cloned and sequenced, and the resulting sequences were assembled. The assembled full-length sequences of the two RNA segments were 6,823 nt (RNA1) and 4,263 nt (RNA2) in length, excluding their poly(A) tails. The complete genome sequences of RNA1 and RNA2 were deposited in the GenBank database (accession nos. MK063924 and MK063925, respectively). Diagnostic primers (HZ-539 5' TGT TAG CAG AGC TAC GTC CTC 3' and HZ-568 5' CCT GAA TCT GCC CAC GAC TT $3^{\prime}$ ) were designed using the Primer3 v. 2.3.7 tool to amplify a partial sequence of RNA2-ORF1 (730 nt) to confirm the presence of this virus in infected plants (Untergasser et al., 2012).

According to the species demarcation criteria proposed by the ICTV Secoviridae Study Group, viruses belonging to different species share less than $80 \%$ aa sequence identity in the protease-polymerase (Pro-Pol) region of the RNA1 polyprotein and less than $75 \%$ aa sequence identity in the coat protein (CP) region (Sanfaçon et al., 2009). The celery virus shares $86.4 \%$ aa sequence identity in the Pro-Pol region and $80.3 \%$ aa sequence identity in the CP region with CaTV1 (Table 1). Based on these criteria, the celery virus should be considered a new strain of CaTV1, for which we propose the name "CaTV1-celery" (isolate JKI-29346). However, further comparison of the CaTV1-celery genome with the reference sequences of CaTV1 revealed considerable differences. 


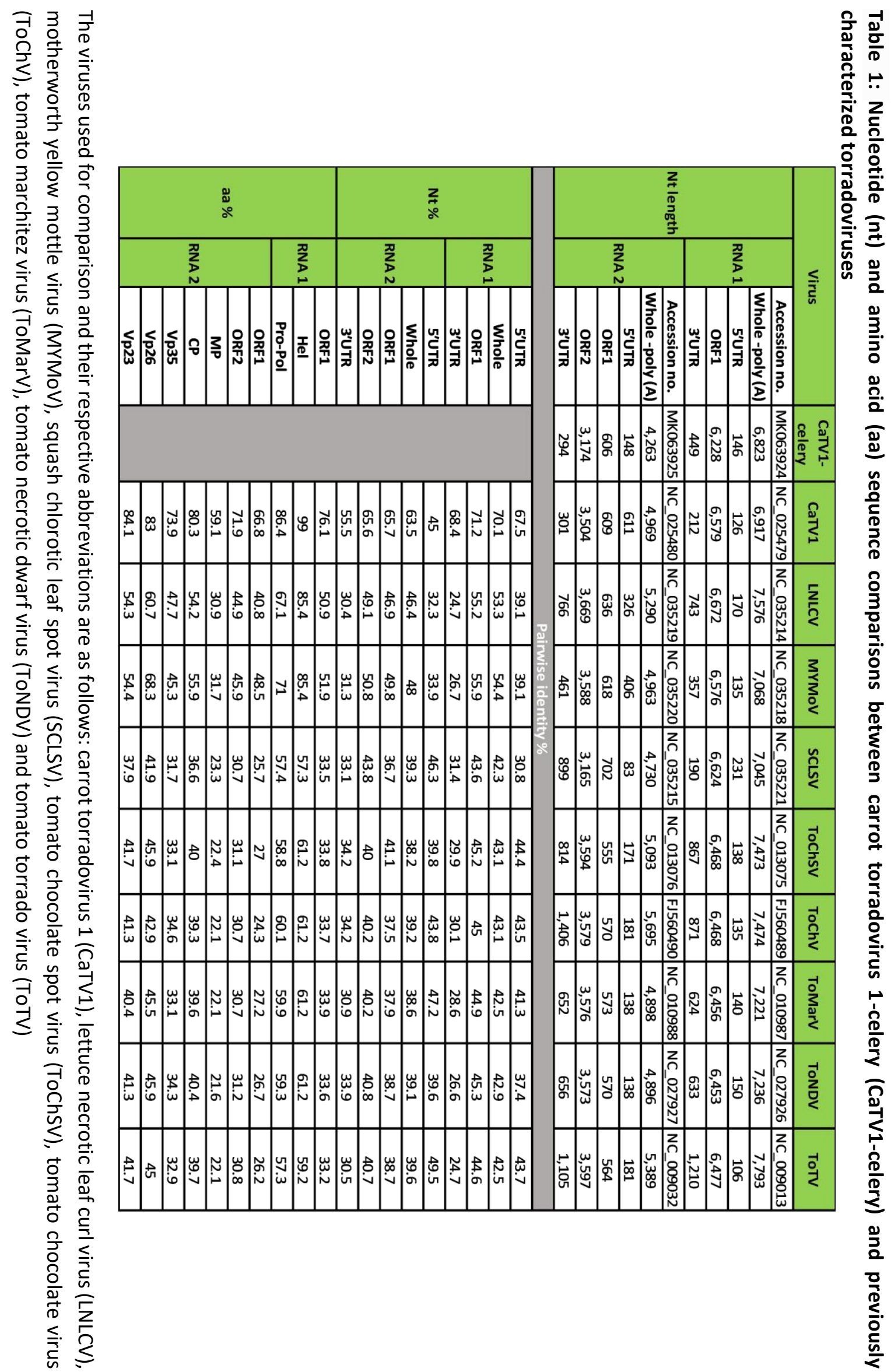


The genomic organization of CaTV1-celery is analogous to that of other torradoviruses (Thompson et al., 2017). Accordingly, RNA1 contains a single open reading frame (ORF1) encoding a polyprotein of 2,076 aa (MW: $237 \mathrm{kDa}$ ). A search of the Conserved Domain Database (CDD) of NCBI identified the presence of two conserved domains: the RNA helicase (Hel) (RNA_helicase; pfam00910) and RNA-dependent RNA polymerase (RdRp) (RNA_dep_RNAP; cd01699) in the RNA1 polyprotein. An additional motif, characteristic of cysteine proteases (3C), was identified using the Geneious search tool (Fig. 1a) (Argos et al., 1984; Gorbalenya et al., 1989; Gorbalenya \& Snijder, 1996). RNA2 is bicistronic, with RNA2 ORF1 encoding a 201-aa protein (MW: $22.0 \mathrm{kDa}$ ) and RNA2 ORF2 encoding a predicted polyprotein of 1,057 aa (MW: $117.1 \mathrm{kDa}$ ). The protein encoded by RNA2 ORF1 did not match any domain in the CDD database. The RNA2 ORF2 polyprotein contains two conserved domains: the $3 A$ movement protein (MP) family domain (3A superfamily; cl02970) and picornavirus capsid protein (CP)-like domain (rhv_like superfamily; cl13999). A motif search identified the position of the MP conserved motif (LxxPXL) in the RNA2 ORF2 polyprotein (Mushegian, 1994; Verbeek et al., 2007). In addition, the MP and the three mature CP subunit peptides were determined based on homologies to those of other torradoviruses (Fig. 1a). 


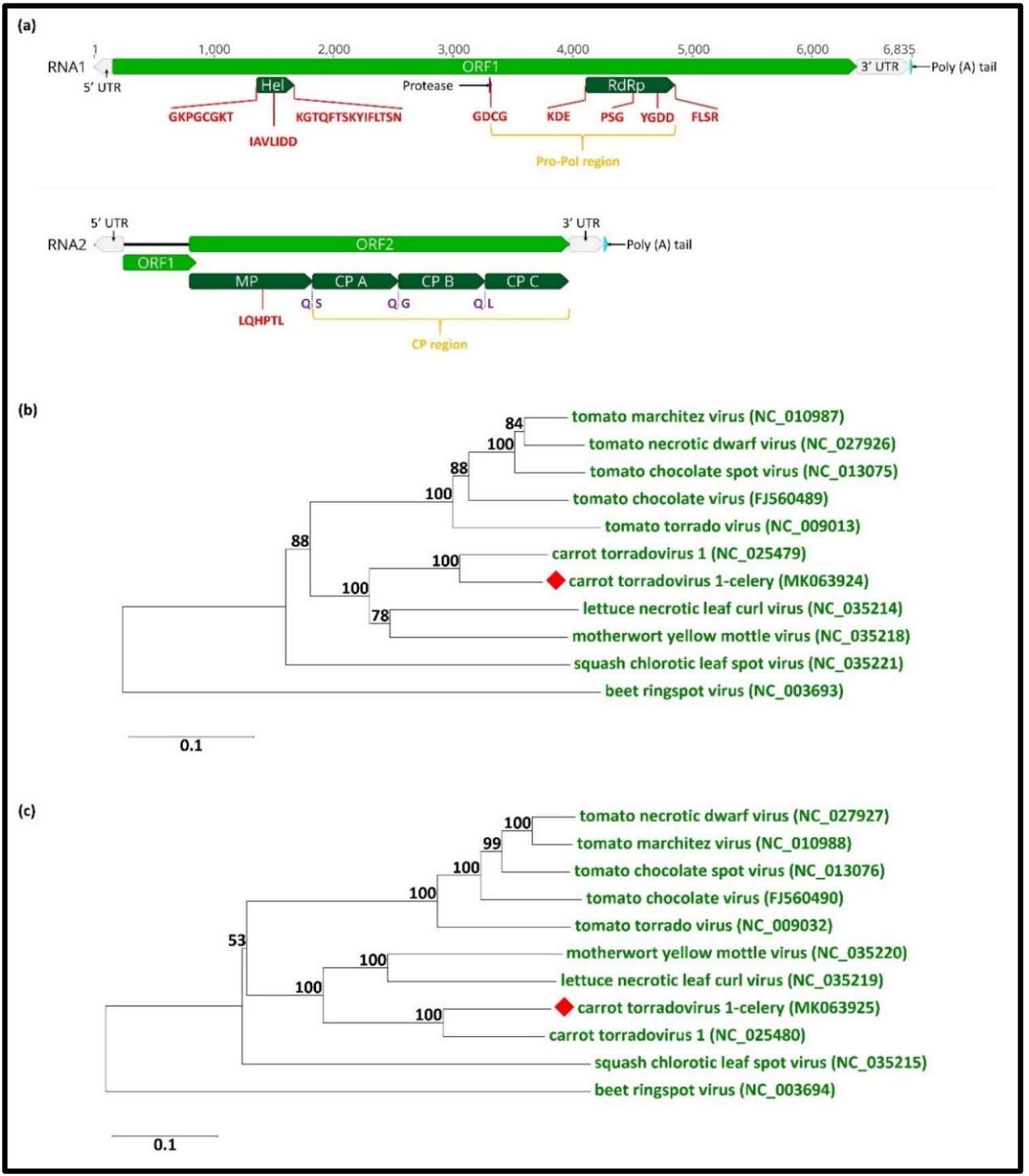

Figure 1: (a) Schematic representation of the carrot torradovirus 1-celery (isolate JKI 29346) genome. RNA1 encodes a polyprotein containing the helicase (Hel), protease and RNAdependent RNA polymerase (RdRp). RNA2 has an ORF1 with unknown function and ORF2 encoding a polyprotein containing the movement protein (MP) and three coat protein subunits $(\mathrm{CP} \mathrm{A}=\mathrm{Vp} 35, \mathrm{CP} \mathrm{B}=\mathrm{Vp} 26$ and $\mathrm{CP} C=\mathrm{Vp} 23)$. Both strands are flanked by $5^{\prime}$ and $3^{\prime}$ untranslated regions (UTR) and are polyadenylated at the $3^{\prime}$ end. The aa sequences of the conserved motifs in Hel, RdRp and MP are shown in red. The predicted cleavage sites are shown in purple. Taxonomically relevant protein segments are highlighted in yellow (b and $\mathbf{c})$. Neighbour-joining trees based on amino acid sequence alignments of the Pro-Pol (b) and CP (c) of CaTV1-celery (red diamond) with those of members of the genus Torradovirus, with beet ringspot virus (genus Nepovirus) as an outgroup. The GenBank accession numbers are shown in brackets. Bootstrap values above $50 \%$ (1000 replicates) are indicated for each node, and the scale bar represents a genetic distance of 0.1 
The percentages of identity in a ClustalW 2.1 pairwise alignment between CaTV1celery RNAs, ORFs and regions with their cognates in other torradoviruses are listed in Table 1. Comparisons of the CP region sequences showed that CaTV1-celery shares between 36.6 and $80.3 \%$ aa sequence identity with torradoviruses. Additionally, the ProPol region of CaTV1-celery shares between 57.3 and $86.4 \%$ aa sequence identity. Neighbour-joining trees based on the aa sequence alignments of the Pro-Pol and CP regions showed that the celery virus clusters together with CaTV1 within the genus Torradovirus (Fig. 1b and c) (Larkin et al., 2007; Kumar et al., 2016).

Despite the observed similarities, the lengths of the $5^{\prime}$ and $3^{\prime}$ untranslated regions (UTR) of RNA1 are 146 and 449 nt long, respectively, and those of RNA2 are 245 and $294 \mathrm{nt}$ long. These values differ from the 5' and 3' UTRs of CaTV1, which are 126 and $212 \mathrm{nt}$ long, respectively, in RNA1 and $611 \mathrm{nt}$ and $301 \mathrm{nt}$ long in RNA2. Additionally, pairwise alignment of the UTRs with those of other torradoviruses, including CaTV1, showed low nt sequence identity values between $24.7 \%$ and $68.4 \%$ (Table 1). The predicted protein encoded by ORF1 of RNA2 of CaTV1-celery shares only $66.8 \%$ aa sequence identity with its homolog in CaTV1, and the 3'UTRs of both segments share $\leq$ 68.4\% nt sequence identity with those of CaTV1. Furthermore, RNAs 1 and 2 of CaTV1celery are shorter by 96 and $706 \mathrm{nt}$, respectively, than their CaTV1 counterparts, making the CaTV1-celery genome the shortest torradovirus genome identified so far, with a total size of $11,086 \mathrm{nt}$, excluding the poly (A) tail (Table 1 ).

Another criterion that is considered for species demarcation in the family Secoviridae is vector specificity. The close evolutionary relationships between CaTV1, CaTV1-celery and lettuce necrotic leaf curl virus (LNLCV) suggest that CaTV1-celery is also an aphid-borne torradovirus (Rozado-Aguirre et al., 2016; Verbeek et al., 2017). To test this, Myzus persicae aphids from a single laboratory clone were reared on CaTV1celery-infected $N$. benthamiana for seven days, after which ten aphids were transferred to three groups of healthy plants (10 N. benthamiana, $10 \mathrm{~A}$. graveolens and $10 \mathrm{D}$. carota). After an inoculation access period of seven days, the plants were treated with the systemic insecticide PIRIMOR (Deutsche $\mathrm{ICI}$ ) according to the manufacturer's instructions. The plants were incubated under greenhouse conditions (at $22{ }^{\circ} \mathrm{C}$; photoperiod of $16 \mathrm{~h}$ light and $8 \mathrm{~h}$ dark) for two months, but no symptoms were observed. The absence of virus infections in acceptor plants was additionally confirmed by negative RT-PCR results. Aphid transmission experiments were repeated three times.

Although CaTV1-celery is considered a divergent strain of CaTV1 based on their aa sequence similarity in the Pro-Pol and CP regions, the data suggest that it might also be useful to consider other molecular criteria for species demarcation, i.e., the total genome size and the size and degree of sequence similarity of the $5^{\prime}$ and $3^{\prime}$ UTR. Taking these criteria into consideration, CaTV1-celery might be accepted in the future as a member of a novel species. Indeed, Sanfaçon and colleagues have already suggested that the current demarcation criteria could be revisited and modified as more viruses become characterized (Sanfaçon et al., 2009). Furthermore, Verbeek and colleagues have proposed additional criteria, i.e., that the aa sequence identity of the RNA2 ORF1 
should be less than $75 \%$ and that the conservation level in the $3^{\prime}$ UTR of both RNAs should be less than $85 \%$ (Verbeek et al., 2010).

Further studies are needed to investigate possible vectors of CaTV1-celery and its potential impact on celery production. Currently, we are developing antibodies against CaTV1-celery as an additional tool for future diagnostic tests and the determination of serological relationships of different torradoviruses. 


\subsection{First report of natural infection of beetroot with beet soil-borne virus}

Yahya Zakaria Abdou Gaafar, A. Sieg-Müller, P. Lüddecke, J. Hartrick, Y. Seide, Jürgen Müller, C. Maaß, S. Schuhmann, K.R. Richert-Pöggeler, A.G. Blouin, S. Massart and H. Ziebell

This article has been published in a slightly modified version as:

Gaafar Y, Sieg-Müller A, Lüddecke, Hartrick J, Seide Y, Müller J, Maaß C, Schuhmann S, Richert-Pöggeler KR, Blouin AG, Massart S, Ziebell H, 2019. First report of natural infection of beetroot with Beet soil-borne virus. New Disease Reports 40, 5. doi: 10.5197/j.20440588.2019.040.005. 
Beetroot (Beta vulgaris subsp. vulgaris) is becoming increasingly popular in Germany with an increase in field production from 1,205 ha in 2013 to 1,826 ha in 2018 (Behr, 2019). It is estimated that EU-wide 24,000 ha of beetroot were produced in 2018 (Behr, 2019). In contrast, sugarbeet was produced on a substantially larger scale with 413,900 ha in Germany in 2018 (Kemper et al., 2019).

A symptomatic beetroot sample was collected in October 2018 from a small field (c. $200 \mathrm{~m}^{2}$ ) in Rhineland-Palatinate, Germany, where several plants displayed virus-like symptoms. The sample submitted displayed necrosis, reduced size and in particular root proliferation (bearding) resembling the symptoms of rhizomania (Fig. 1). However, immunosorbent electron microscopy (ISEM) examination using various antibodies raised against the following beet viruses was not successful in identifying any causal agent: beet black scorch virus, beet curly top virus, beet necrotic yellow vein virus, beet mosaic virus, beet oak-leaf virus, beet soil-borne virus (BSBV), beet soil-borne mosaic virus, beet virus $Q$, beet western yellows virus, beet yellows virus and tobacco rattle virus.

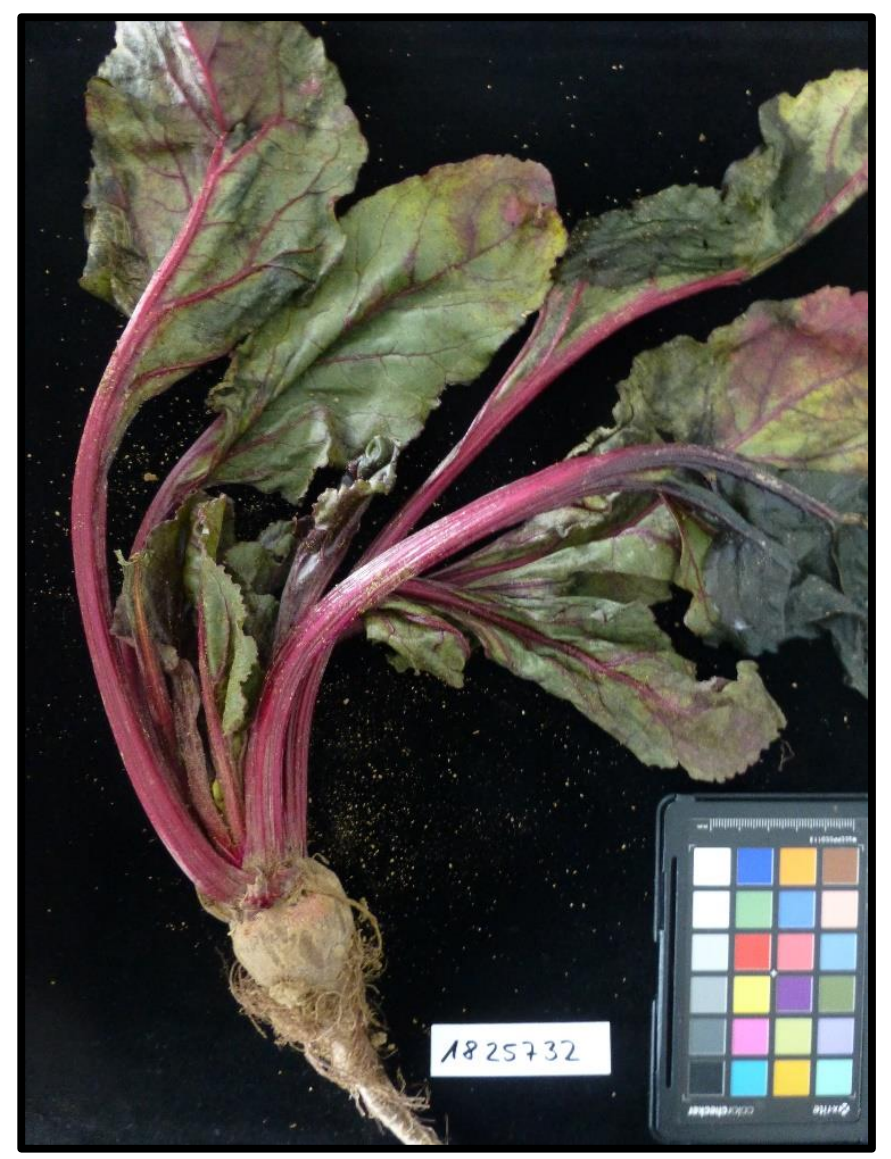

Figure 1: Beetroot sample infected with beet soil-borne virus and beet cryptic virus-2 showing leaf necrosis, reduced size and root bearding.

Total RNA was extracted from the infected beetroot sample using innuPREP RNA Mini Kit (Analytik Jena AG, Germany). Ribosomal RNA was depleted using RiboMinus Plant Kit for RNA-Seq (ThermoFisher Scientific, USA). A library was prepared using TrueSeq Stranded mRNA kit (Illumina, USA). The sequencing was done on a NextSeq 500 platform 
(2×150). The generated data was analysed on Geneious Prime (2019.1.1). The raw reads were quality-trimmed then de novo assembled using SPAdes assembler (3.10.1) (Bankevich et al., 2012). The contigs were compared against a local virus reference database using tBlastx. Twenty-one contigs showed only similarities to BSBV and Beet cryptic virus-2 (BCV-2), respectively; no other virus sequences were found in the data.

BSBV is a member of the Pomovirus genus (family Virgaviridae) (Adams et al., 2017). The virus is widely distributed in sugar beet growing areas causing yield losses. BCV2, a member of the Deltapartitivirus genus (family Partitiviridae), is a symptomless virus that is also common in sugar beet (Antoniw et al., 1990; Vainio et al., 2018). For confirmation of BSBV infection, total RNA was re-extracted from the infected beetroot sample and RT-PCR was performed using two specific primer pairs targeting the RNAdependent RNA polymerase and movement protein regions of BSBV, respectively ( $\mathrm{HZ772}$ 5'-GTTGGTGTTGGTCAGTTGGC-3' / HZ773 5'-TGGTCAACGGCGAAATCAGA-3' and HZ774 5'GAGGGGTAAGACACAGCGAC-3' / HZ775 5'-CACTTCGTCCTCCTGGTCAC-3'). Two bands of the expected sizes (923 and $766 \mathrm{bp}$, respectively) were produced.

The almost complete genomes of BSBV and BCV-2 were assembled by Geneious mapping using reference sequences (BSBV: Genbank Accession Nos NC_003518NC_003520 and BCV-2: NC_038845-NC_038847). The sequences of the beetroot BSBV and BCV-2 isolates were submitted to Genbank (MK731954-MK731959). Sequence analysis revealed that the BSBV isolate shares $97.3-98.5 \%$ nucleotide identity with the reference genome (German isolate NC_003518-NC_003520). The BCV-2 isolate shares 98.7-99.7\% nt nucleotide identity with the reference genome (Hungarian isolate NC_038845-NC_038847).

This work provides the first suggestion that BSBV naturally infects beetroot. This identification exposes the limit of diagnostic methods such as ISEM, possibly due to low titre or the existence of a divergent virus isolate, and also highlights the strength of high throughput sequencing to rapidly and accurately diagnose plant viruses. Furthermore, these findings demonstrate that high value crops such as beetroot might be affected by pathogens of major crops and therefore should be considered in crop rotations. 


\subsection{First report of physostegia chlorotic mottle virus on tomato (Solanum lycopersicum) in Germany}

Yahya Zakaria Abdou Gaafar, M. A. M. Abdelgalil, D. Knierim, K. R. Richert-Pöggeler, W. Menzel, S. Winter and H. Ziebell

This article has been published in a slightly modified version as:

Gaafar YZA, Abdelgalil MAM, Knierim D et al., 2018. First report of physostegia chlorotic mottle virus on tomato (Solanum lycopersicum) in Germany. Plant Disease 102, 255. https://doi.org/10.1094/PDIS-05-17-0737-PDN 
In September 2015, a tomato sample collected in the German state of Hesse was sent to the Julius Kühn-Institut for analysis. While the fruits showed marbling and discoloration, the leaf samples from this plant did not show any obvious symptoms (Fig. 1). Transmission electron microscopy (TEM) revealed the presence of bullet-shaped virus particles indicating the presence of a rhabdovirus (Fig. 2). However, immunosorbent electron microscopy using antiserum JKI-1073 for Eggplant mottled dwarf virus (EMDV) could not confirm EMDV infection. The virus was mechanically transmitted to Nicotiana benthamiana, N. clevelandii, and Chenopodium quinoa inducing yellowing and leaf deformation, while mechanical transmission to $N$. occidentalis (P1 and 37b) failed. Extraction of double stranded-RNA (dsRNA) followed by random-PCR (Froussard, 1992), cloning of PCR products, and sequencing failed to reveal any virus sequences.

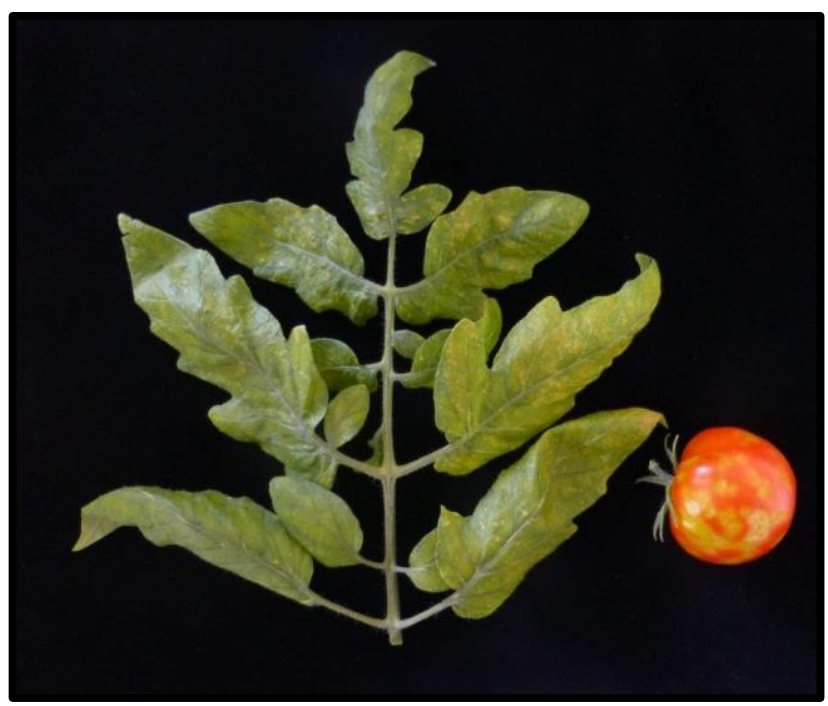

Figure 1: Tomato fruit showing marbling, discoloration and leaf distortion whereas leaf symptoms consist of mild yellow spots.

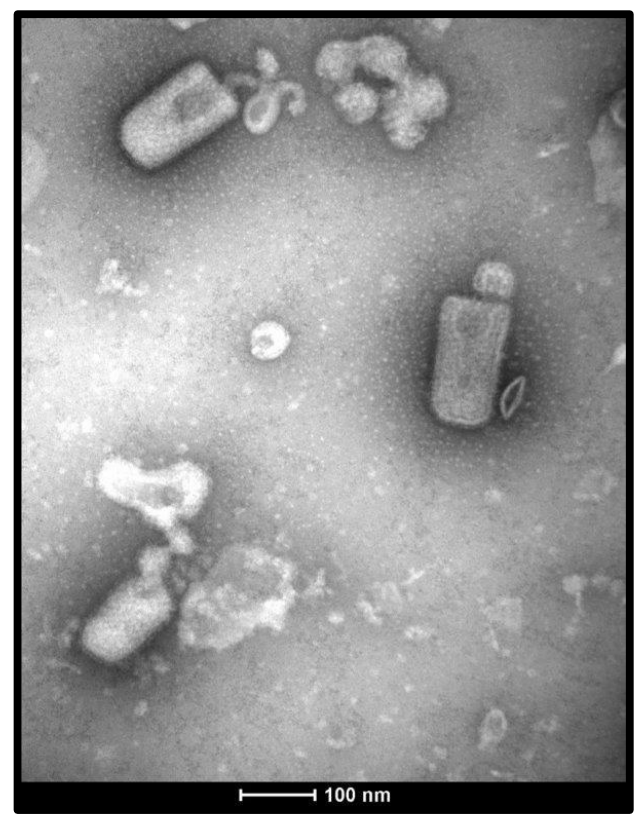

Figure 2: Electron microscopy photograph of physostegia chlorotic mottle virus. 
Total RNA was extracted from infected $N$. benthamiana, followed by ribodepletion, library preparation and submission for next-generation sequencing (NGS) using an Illumina MiSeq platform as described by (Knierim et al., 2017). De novo assembly of the trimmed reads was done with Geneious v 10.1.3 (Biomatters LTD, NZ). Using MEGA BLAST, 13 contigs showed between 95.6 and $98.5 \%$ similarity with physostegia chlorotic mottle virus (PhCMoV) isolate PV-1182 (accession no. KX636164). The complete PhCMoV genome (13,321 nt length) was assembled by mapping reads to this reference genome and used to design PhCMoV-specific RT-PCR primers for detection (HZ-343 5' CGG TGA GTG GGG CAA CTA AT $3^{\prime}$ and HZ-344 5' AGC GAT GGG GTC TAG TGT CT 3'). RT-PCR confirmed the presence of PhCMoV in the test plants resulting in amplicons of approximately 875 bp.

In August 2016, similar symptoms on tomato fruits were observed by a different grower in Hesse. The presence of PhCMoV was confirmed by TEM and RT-PCR. Additionally, the PCR products were sequenced and showed $97 \%$ identity to KX636164. Surprisingly, reanalysis of a tomato sample from 2003 that was infected by a hitherto unknown rhabdovirus using NGS also confirmed infection with PhCMoV. This sample also originated from Hesse although the original grower is unknown. The complete genome of the $2003 \mathrm{PhCMoV}$ sample was assembled following the same methods described above. Pairwise comparison between the genomes of 2015 and 2003 isolates resulted in $99.7 \%$ nucleotide identity and $96.9 \%$ when compared with KX636164.

These findings indicate the presence of PhCMoV in tomato in Germany for a long time albeit isolated occurrences in different production areas. PhCMoV was recently identified from Physostegia virginiana plants showing leaf deformation and severe chlorotic and mottle symptoms in Austria (Menzel et al., 2016). However, it is not known if there is a link between $\mathrm{PhCMoV}$ isolates infecting $P$. virginiana and tomato as the routes of transmission and dissemination are currently unknown. The sequences from this report were deposited in GenBank (accession nos. KY706238 and KY859866 [full-length sequences], KY882263 and KY882264 [partial sequences]). To our knowledge, this is the first host record of PhCMoV in tomato and a new country record for Germany. 


\subsection{First report of turnip crinkle virus infecting garlic mustard (Alliaria petiolata) in Germany}

Yahya Zakaria Abdou Gaafar, A. Sieg-Müller, P. Lüddecke, K. Herz, J. Hartrick, C. Maaß, S. Schuhmann, K. R. Richert-Pöggeler and H. Ziebell

This article has been published in a slightly modified version as:

Gaafar Y, Sieg-Müller A, Lüddecke P, Herz K, Hartrick J, Maaß C, Schuhmann S, RichertPöggeler KR, Ziebell H, 2019. First report of Turnip crinkle virus infecting garlic mustard (Alliaria petiolata) in Germany. New Disease Reports 39, 9. http://dx.doi.org/10.5197/j.20440588.2019.039.009 
In May 2018, three samples of wild garlic mustard (Alliaria petiolata, Brassicaceae) were collected from a private garden in Koenigslutter, Germany. While sample EPV_18_002 was asymptomatic apart from slight yellowing, samples EPV_18_003 and EPV_18_004 showed stunting, yellowing, necrosis and severe crinkling (Figs. 1-3). It was possible to mechanically transmit the suspected virus from all three samples to Nicotiana benthamiana; the same systemic symptoms of leaf crinkling, rolling and yellowing appeared seven days post infection on all plants, and the plants died in the second week. Infected $N$. benthamiana leaves were analysed by electron microscopy and icosahedral particles of c. $30 \mathrm{~nm}$ in diameter were observed that reacted with antibodies (Julius Kuehn Institute, reference number JKI-1177) raised against a UK isolate of turnip crinkle virus (TCV) (Fig. 4).
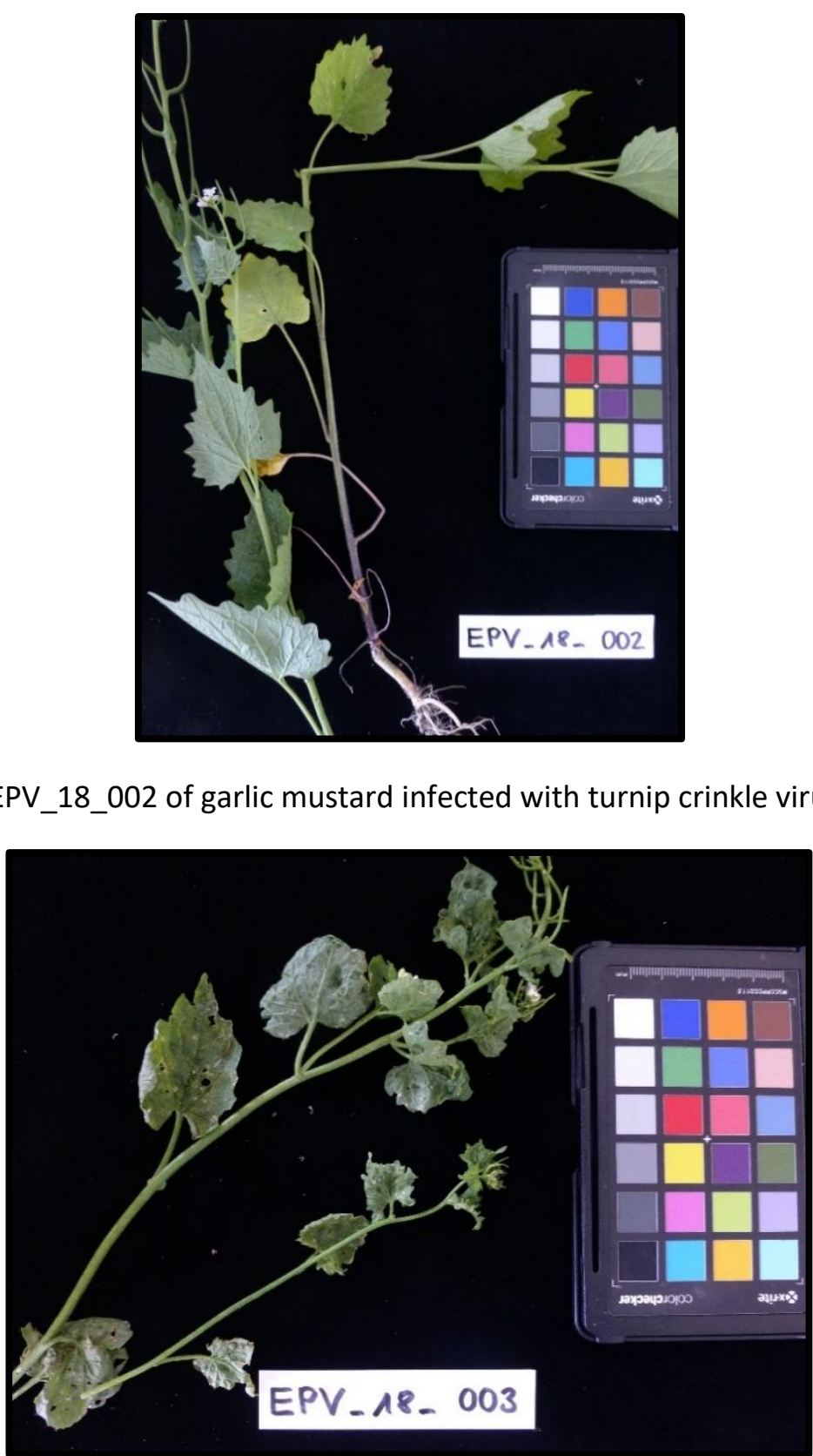

Figure 2: Sample EPV_18_003 of garlic mustard infected with turnip crinkle virus. 


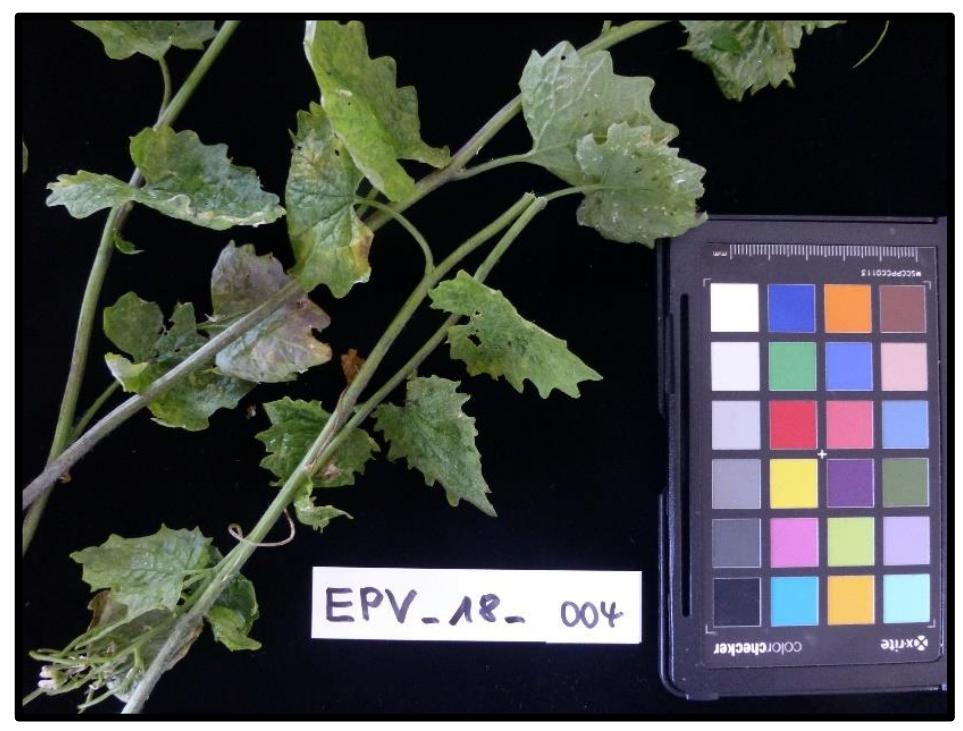

Figure 3: Sample EPV_18_004 of garlic mustard infected with turnip crinkle virus.

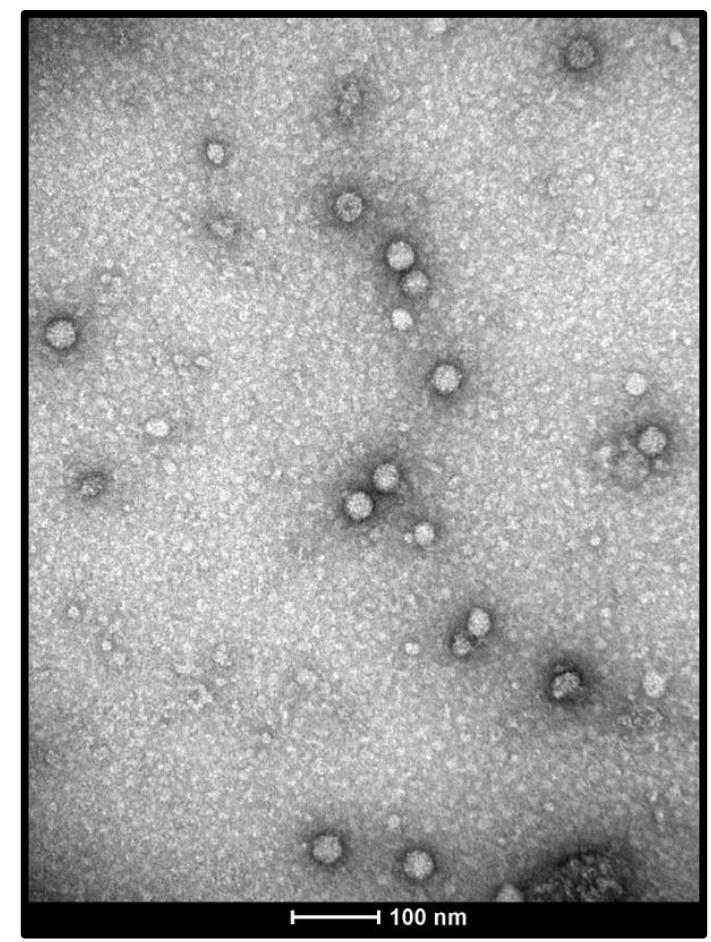

Figure 4: Electron microscope image of turnip crinkle virus (TCV) from infected Nicotiana benthamiana leaves. Particles were decorated using a TCV-specific antiserum (JKI-1177).

To obtain the full viral genome, dsRNA was extracted from symptomatic $N$. benthamiana leaves (inoculated from sample EPV_18_002) using a Viral dsRNA Extraction Mini Kit for Plant Tissue (iNtRON, South Korea) and sent for library preparation and sequencing on Illumina NovaSeq 6000 platform (2x150 bp) at Eurofins GATC Biotech $\mathrm{GmbH}$, Germany. Using Geneious Prime (2019.0.4), the raw reads were quality trimmed, 
filtered, error corrected and normalised, followed by de novo assembly. A BLASTn search of the generated contigs confirmed the presence of eight contigs (131 to 4,057nt) with nucleotide (nt) identities of $82.3 \%$ to $86.7 \%$ to TCV (NC_003821); there was no indication of the presence of satellite RNAs. The full genome of the German TCV isolate (JKI ID 29306) was assembled using this reference genome. The complete genome of TCV-JKI-29306 was 4,061 nt (accession no. MK301398). The presence of TCV was confirmed in all three original samples by RT-PCR using OneTaq One-Step RT-PCR Kit (NEB, USA) with the primer pair (HZ632 5' AAA GGC AAA ACT GGG TGG GA 3' and HZ633 5' TAA AGT TTG CGG CTA GGG G 3') generating a 339 bp fragment.

In further comparisons using MUSCLE (3.8.425, (Edgar, 2004)), the German TCV isolate shared $82.8 \%$ nt identity to NC_003821 and $82.6 \%$ to AY312063 (Table 1). Additionally, the protein sequences of the different TCV genes shared identities of 75.0 to 90.7\% to the proteins of NC_003821 and AY312063 (Table 1). According to ICTV criteria, these data indicate the presence of a novel TCV strain (Rochon et al., 2012).

Table 1. Pairwise identities of the turnip crinkle virus (TCV) strain from garlic mustard in Germany with whole TCV genomes from the UK using a multiple sequence alignment tool, MUSCLE.

\begin{tabular}{|c|c|c|c|c|c|c|}
\hline \multirow{2}{*}{$\begin{array}{c}\text { Accession } \\
\text { no. }\end{array}$} & Genome & \multicolumn{5}{|c|}{ Amino acid identity (\%) } \\
\cline { 5 - 8 } & $\begin{array}{c}\text { nucleotide } \\
\text { identity (\%) }\end{array}$ & $\begin{array}{c}\text { p88 } \\
\text { RP }\end{array}$ & $\begin{array}{c}\text { p28 } \\
\text { ARP }\end{array}$ & $\begin{array}{c}\text { p8 } \\
\text { MP }\end{array}$ & $\begin{array}{c}\text { p9 } \\
\text { MP }\end{array}$ & $\begin{array}{c}\text { p38 } \\
\text { CP }\end{array}$ \\
\hline NC_003821 & 82.8 & 90.5 & 84.4 & 75.0 & 89.4 & 85.5 \\
\hline AY312063 & 82.6 & 90.7 & 84.8 & 76.4 & 88.2 & 85.2 \\
\hline
\end{tabular}

TCV belongs to the genus Betacarmovirus (family Tombusviridae). It infects members of the Brassicaceae family causing crop losses (Broadbent \& Heathcote, 1958; Lister, 1958). To our knowledge, this is the first report of a TCV strain from Germany occurring on garlic mustard. Although TCV is one of the model organisms in plant virology and therefore well studied, there is surprisingly little literature available on occurrence, host range and impact of this virus. As the original host plants appeared as weeds in a private garden (approximately 40 plants with a minority displaying symptoms), the impact of TCV in Germany and other countries on cultivated crop plants is currently unknown. 


\subsection{First report of southern tomato virus in German tomatoes}

Yahya Zakaria Abdou Gaafar, P. Lüddecke, C. Heidler, J. Hartrick, A. Sieg-Müller, C. Hübert, A. Wichura and H. Ziebell

This article has been published in a slightly modified version as:

Gaafar Y, Lüddecke P, Heidler C et al., 2019. First report of Southern tomato virus in German tomatoes. New Disease Reports 40, 1. doi: 10.5197/j.2044-0588.2019.040.001. 
Southern tomato virus (STV) is a member of the genus Amalgavirus (family: Amalgaviridae). It has been identified in tomatoes (Solanum lycopersicum) in several countries in Asia, Europe, and North and South America (Sabanadzovic et al., 2009; Candresse et al., 2013; Padmanabhan et al., 2015). Its genome is composed of a dsRNA of $\sim 3.5 \mathrm{~kb}$. STV is known to be transmitted through seed at high rates (Sabanadzovic et al., 2009).

In 2019, greenhouse tomatoes from Lower Saxony, Germany, showed symptoms consisting of mottling, yellowing and/or chlorotic spots (Figs. 1-2). Eight samples were sent to the Julius Kuehn Institute for analysis. As an infection with Tomato brown fruit rugose virus was suspected, the samples were analysed by high throughput sequencing on a MinION sequencer (Oxford Nanopore Technologies, UK) for rapid diagnosis. Briefly, two samples were pooled, and dsRNA extracted from $100 \mathrm{mg}$ leaf material using the Viral dsRNA Extraction Mini Kit for Plant Tissue (iNtRON, South Korea). Random cDNA was synthesised using ProtoScript II Reverse Transcriptase (NEB, USA) and 8N random primers preceded by a denaturation step at $99^{\circ} \mathrm{C}$ for two minutes. Second strand synthesis was done using a NEBNext Ultra II Non-Directional RNA Second Strand Synthesis Module kit (NEB). The samples were end-repaired using the NEBNext End Repair module (NEB), dA tailed with NEBNext dA-tailing module (NEB) and the four pools barcoded by native barcoding followed by adaptor ligation (Oxford Nanopore Technologies, UK) according to the manufacturers' instructions. All purification steps were performed using a Mag-Bind TotalPure NGS kit (Omega Bio-Tek, USA). The libraries were mixed and loaded to a MinION flow cell and sequenced for 16 hours using a MinION sequencer connected to a computer with MinION software (r18.12.9; ONT).

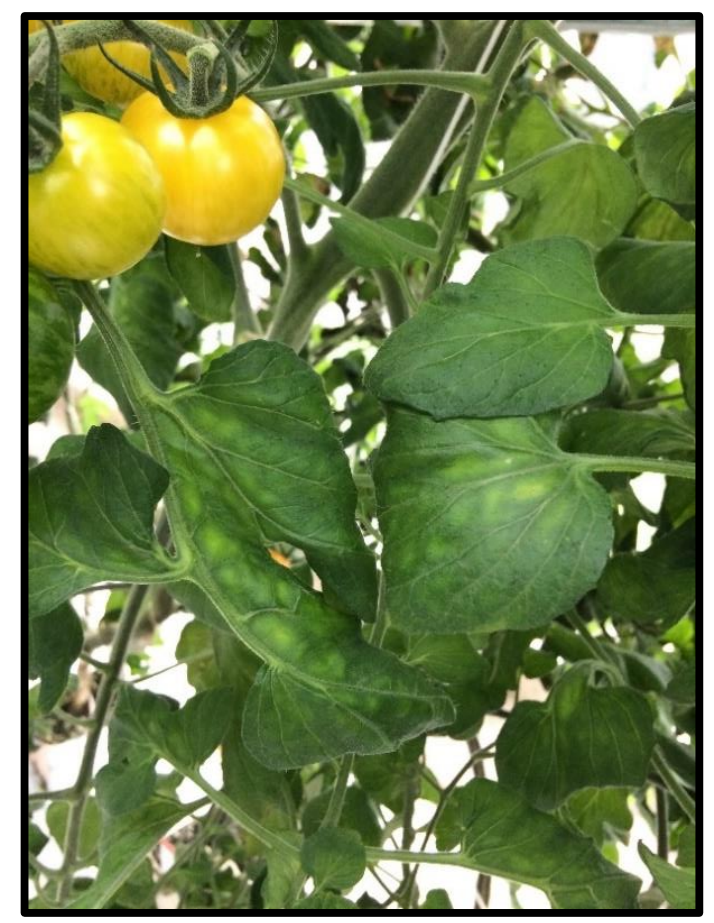

Figure 1: Chlorotic lesions observed on diseased tomato plants grown in Lower Saxony. 


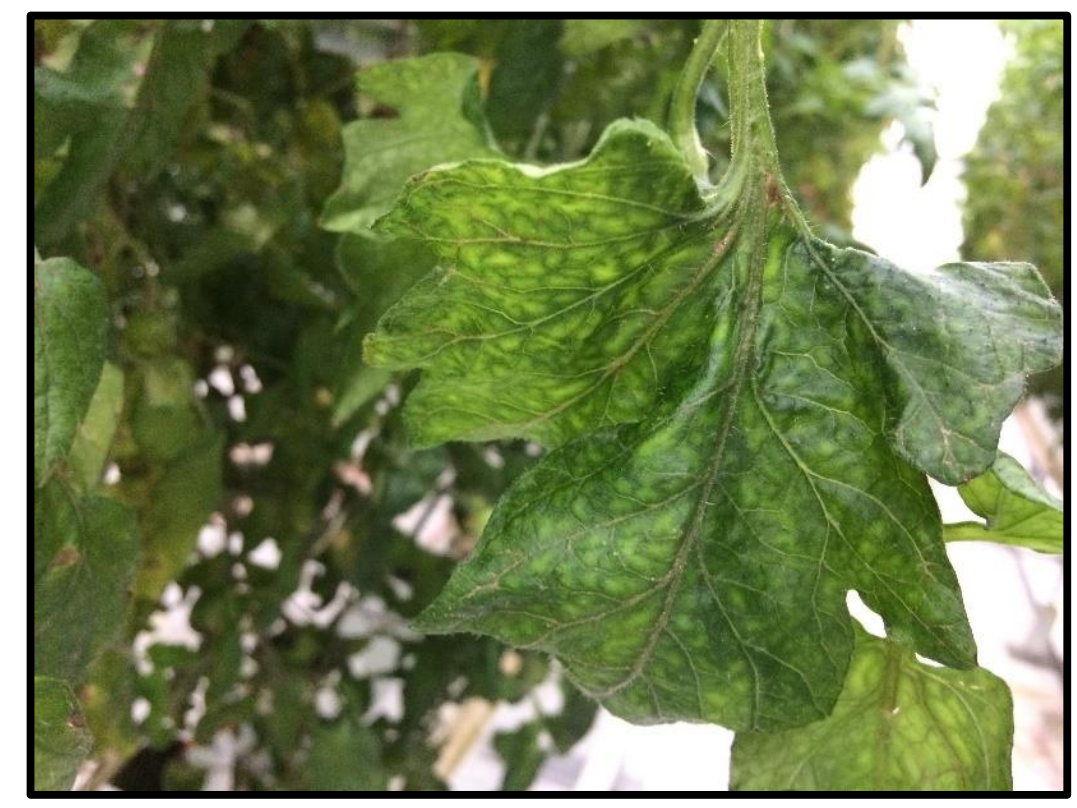

Figure 2: Severe chlorotic lesions and mottling of leaves on diseased tomato plants grown in Lower Saxony.

The reads were basecalled and barcode-splitting was done using the Guppy toolkit (v2.3.7; ONT). De novo assembly of reads was done using Canu (v1.8) (Koren et al., 2017). The unassembled reads and assembled contigs were Blastn searched against a local GenBank nt database using Blast+ (v2.9.0) and visualised with Blast Viewer (v5.2.0) (Durand et al., 1997; Camacho et al., 2009). STV sequences were detected in two of the pools, and no other virus sequences were detected. The full genomes were assembled by mapping to STV reference (Genbank Accession no. NC_011591) using mapping to reference tool on Geneious Prime (v2019.1.3). The sequences had 99.9\% nt identity to each other and to STV isolate CH_bpo 163 from Switzerland (MF422618).

To confirm the findings in the original samples, total RNA was extracted for the four samples in the positive pools using an innuPREP RNA MiniKit (Analytik Jena AG, Jena, Germany), and RT-PCR was performed using a primer pair (HZ782 5'CAAGTGGGCCGTTTCTTTGG-3' and HZ783 5'-TGAAGACCGCCTGGAAAGTC-3'). STV infection was confirmed in three samples. The RT-PCR products were purified, and Sanger sequenced at Eurofins Genomics (Germany). The sequences had 100\% identity to the sequences from the MinION. The genomes of the two pools were submitted to Genbank (MK948544 and MK948545). To our knowledge, this is the first report of STV infecting tomato in Germany. The study also shows the potential to use Minlon technology for rapid detection and identification of virus sequences. 


\subsection{Two divergent isolates of turnip yellows virus from pea and rapeseed and first report of turnip yellows virus- associated RNA in Germany}

Yahya Zakaria Abdou Gaafar and H. Ziebell

This article has been published in a slightly modified version as:

Gaafar YZA, Ziebell H, 2019. Two divergent isolates of turnip yellows virus from pea and rapeseed and first report of turnip yellows virus-associated RNA in Germany. Microbiology Resource Announcements 8, 2254. doi: 10.1128/MRA.00214-19. 


\subsubsection{Abstract}

Two divergent isolates of turnip yellows virus (TuYV) were identified in pea and rapeseed. The nearly complete genome sequences of the virus isolates share $93.3 \%$ nucleotide identity with each other and $89.7 \%$ and $92.9 \%$ with their closest isolate from South Africa. Additionally, a turnip yellows virus-associated RNA was identified.

\subsubsection{Main text}

Turnip yellows virus (TuYV), the non-sugar beet-infecting strain of beet western yellows virus (BWYV), is a polerovirus (family Luteoviridae) (Mayo, 2002; Graichen \& Rabenstein, 1996). TuYV can infect a wide range of crops, predominantly members of the Brassicaceae and Fabaceae families.

Two plant samples (pea [Pisum sativum] and oilseed rape [Brassica napus]) displaying yellowing symptoms were collected in Germany (in 2016 and 2006, respectively). The samples were tested with a triple antibody sandwich enzyme-linked immunosorbent assay (TAS-ELISA) for the presence of luteoviruses/poleroviruses using monoclonal antibodies 5G4 and 6G4 (Katul, 1992), as described (Abraham et al., 2006; Gaafar et al., 2016). Both samples tested positive for polerovirus infection but showed different titers, which prompted us to determine the genomic sequences of these isolates. The viruses were maintained on pea (isolate identifier [ID] JKI 29344) and radish (JKI 29345) by aphid transmission using Myzus persicae. Nonviruliferous aphids were left to feed for 3 days on the infected leaves, and then 10 aphids were transferred to healthy plants for 3 days ( 3 cycles for the pea isolate and 33 cycles for the oilseed rape isolate). Four weeks post-aphid inoculation, polerovirus infection of plants was confirmed using TAS-ELISA and reverse transcription-PCR (RT-PCR) with generic polerovirus primers (S2 and AS3) (Abraham et al., 2007). The RT-PCR bands were Sanger sequenced, and a BLASTn search resulted in the highest hits, with $99 \%$ (pea isolate) and $100 \%$ (oilseed rape isolate) nucleotide identities to the partial coat protein sequences of BWYV (GenBank accession no. L39976) and TuYV (GenBank accession no. KU198395).

For genome sequencing, total RNAs were extracted with the innuPREP RNA minikit (Analytik Jena AG), followed by rRNA depletion with the RiboMinus plant kit (Invitrogen). cDNAs were synthesized using ProtoScript II reverse transcriptase (NEB) and random octanucleotide primers, followed by second-strand synthesis with the NEBNext Ultra II nondirectional RNA second-strand synthesis module kit (NEB). The libraries were prepared using a Nextera XT library kit (Illumina) and submitted for high-throughput sequencing (HTS) on the MiSeq version 3 platform $(2 \times 301)$. The raw reads (total reads, 1,640,360 for JKI 29344 and 1,648,784 for JKI 29345) were analyzed using the Geneious software (11.1.4). The reads were quality trimmed (error limit, 0.05) and size filtered to $>99$ nucleotides (nt), followed by de novo assembly using the Geneious 
assembler (parameter, medium sensitivity/fast). The assembled contigs were used to search the NCBI database using BLASTn. A contig of about $5.6 \mathrm{~kb}$ in each sample showed 90.7\% and 93.1\% nt identities to TuYV (GenBank accession no. KU198395). Contig extension using Geneious mapping to the reference tool (parameter, medium sensitivity/fast) resulted in the complete coding sequences and almost-full-genomic sequences for both TuYV isolates; they shared $93.3 \% \mathrm{nt}$ identity to each other while sharing $89.7 \%$ and $92.9 \%$, respectively, to the most closely related isolate, KU198395.

Pairwise comparisons of the amino acid sequences using MUSCLE (3.8.425) showed that some open reading frames (ORFs) are also highly divergent (P0 and P1), whereas others are not (CP and MP) (Table 1) (Edgar, 2004). The pea and the oilseed rape isolates' ORFs shared between $80.9 \%$ and $99.5 \%$ amino acid (aa) identities to each other and between $74.1 \%$ and $95.6 \%$ in comparison to their homologues of KU198395.

Table 1: Pairwise amino acid comparisons between the predicted proteins of the German TuYV isolates and their homologues of KU198395 using MUSCLE 3.8.425

\begin{tabular}{|c|c|c|c|c|c|c|c|}
\hline $\begin{array}{c}\text { Protein } \\
\text { source } \\
\text { (isolate) }\end{array}$ & \multicolumn{6}{|c|}{ Amino acid identity (\%) by open reading frame(s) } & \multirow{2}{*}{$\begin{array}{c}\text { Reference isolate or } \\
\text { accession no. }\end{array}$} \\
\cline { 2 - 8 } & P0 & P1-P2 & P1 & P3-P5 & CP & MP & JKI 29345 \\
\hline $\begin{array}{c}\text { Pea } \\
\text { (JKI 29344) }\end{array}$ & 80.9 & 90.6 & 86.5 & 97.8 & 99.5 & 99.4 & KU198395 \\
\hline $\begin{array}{c}\text { Oilseed rape } \\
\text { (JKI 29345) }\end{array}$ & 85.9 & 99.8 & 85.3 & 94.1 & 93.1 & 90.9 & KU198395 \\
\hline
\end{tabular}

An additional contig of about $2.8 \mathrm{~kb}$ was found in the oilseed rape sample that shared $98 \%$ nt identity with the partial sequence of beet western yellows virus-associated RNA (BWYVaRNA) from the United Kingdom (GenBank accession number KF533709) (Adams et al., 2014). Polerovirus-associated RNAs are single-stranded RNAs (ssRNAs) of $\sim 2.8$ to $3 \mathrm{~kb}$ and have two major ORFs. They replicate autonomously and appear to depend on a helper virus for aphid transmission by encapsidating within the virus coat protein (Briddon et al., 2012). They may increase the severity of disease symptoms. The full genome of $2,841 \mathrm{nt}$ was assembled by mapping to the reference sequence with NCBI RefSeq accession no. NC_004045 (Chin et al., 1993), and we propose the name "turnip yellows virus-associated RNA" (TuYVaRNA) for this RNA. While TuYVaRNA shares 93\% nucleotide identity with NC_004045 and 98\% with the partial sequence from the United Kingdom, its genomic organization was similar to that of the other polerovirus-associated RNAs, containing three ORFs, with the first one containing an amber readthrough ORF.

To our knowledge, these are the first complete coding sequences of TuYV and the first report of TuYVaRNA from Germany. 


\subsubsection{Data availability}

The complete coding sequences of the two German TuYV isolates and the full sequence of TuYVaRNA can be found in NCBI GenBank under accession numbers MK450519, MK450520, and MK450521. Raw sequence data are available in the Sequence Read Archive (SRA) under BioProject accession number PRJNA524397 and under BioSample accession numbers SAMN11026350 and SAMN11026351. 


\subsection{Vicia faba, V. sativa and Lens}

\section{culinaris as new hosts for pea necrotic}

yellow dwarf virus in Germany and

\section{Austria}

Yahya Zakaria Abdou Gaafar, S. Grausgruber-Gröger and H. Ziebell

This article has been published in a slightly modified version as:

Gaafar Y, Grausgruber-Gröger S, Ziebell H, 2016. Vicia faba, V. sativa and Lens culinaris as new hosts for Pea necrotic yellow dwarf virus in Germany and Austria. New Disease Reports 34, 28. doi: 10.5197/j.2044-0588.2016.034.028 
Pea necrotic yellow dwarf virus (PNYDV) was identified in green peas (Pisum sativum) in Germany in 2009 (Grigoras et al., 2010a). In subsequent years, sampling of symptomatic green peas showed that PNYDV was restricted to Saxony and Saxony-Anhalt (Ziebell, 2015). In Austria, PNYDV was detected in 2010, also in P. sativum (Grigoras et al., 2014).

A countrywide outbreak of virus-like disease symptoms on faba beans (Vicia faba) was reported in Germany in 2016. Many fields had large patches of yellowish and dwarfed plants (Fig. 1). More than 460 samples of $P$. sativum (green and protein peas) and $V$. faba showing virus-like symptoms (Fig. 2) were analysed using ELISA for Alfalfa mosaic virus, Cucumber mosaic virus, Pea enation mosaic virus (PEMV), Red clover vein mosaic viruslike carlaviruses, and luteo-/poleroviruses, nanoviruses and potyviruses. PEMV was the predominant virus found (70.5\% of samples) but infection with luteo-/poleroviruses $(26.7 \%)$, potyviruses $(4.6 \%)$ and carlaviruses $(0.9 \%)$ was confirmed. More importantly, $54.7 \%$ of samples tested positive with an ELISA designed for broad detection of nanoviruses (Grigoras et al., 2010a; Abraham et al., 2012). The same samples did not react with an ELISA designed to detect only Faba bean necrotic stunt virus and Faba bean necrotic yellows virus, indicating infection with PNYDV. Using PCR with PNYDV-specific primers priPeaSdir (5' AAC CTC CGG ATA TCA CCA GAT 3') and priPeaSrev (5' CCG GAG GTT TTA TTT CAA AAC CAA $C 3^{\prime}$ ) targeting the coat protein encoding component $S$ of the genome (T. Timchenko, pers. comm.), PNYDV infection was confirmed for a subset of 18 samples. Sequencing of amplicons showed 98.7 to $99.9 \%$ nucleotide identity with PNYDV (GenBank accession no. JN133279). Three lentil (Lens culinaris) samples from a field trial in central Germany also tested positive for PNYDV using differentiating monoclonal antibodies with confirmation by PCR and sequencing. Sequences from this study can be accessed under accession numbers KY191024 - KY191044.

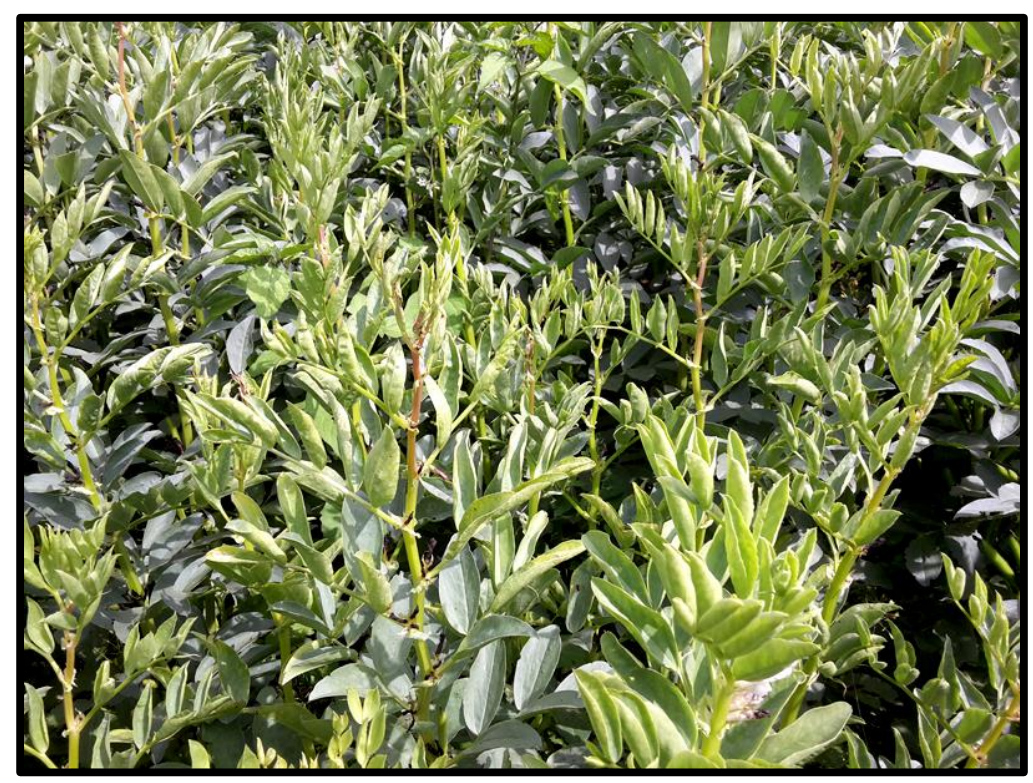

Figure 1: Faba bean crop with a typical patch of virus-infected plants. In particular, yellowing, dwarfing and leaf deformation on the upper parts of the plants indicate nanovirus infection. 


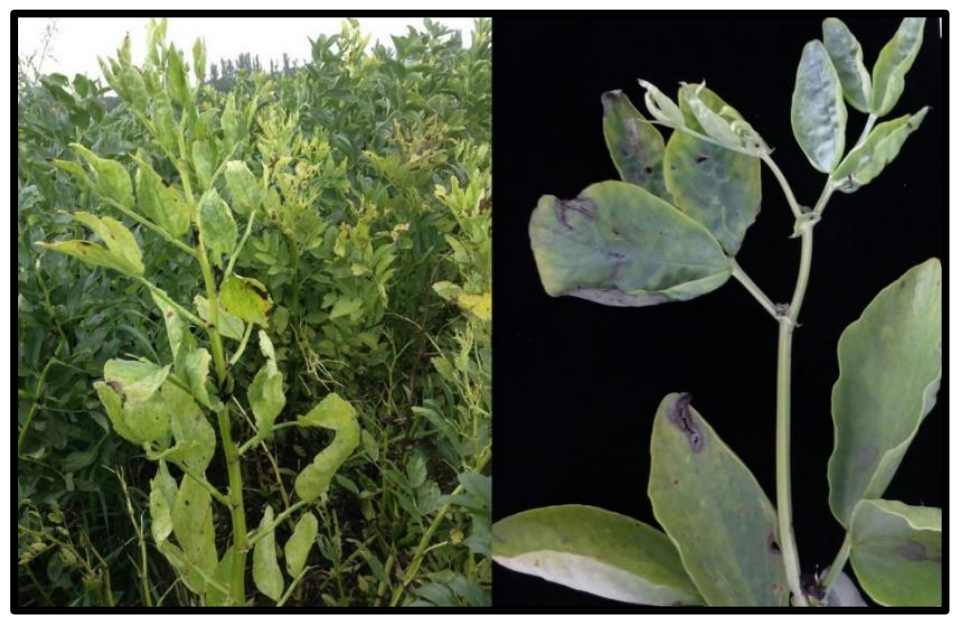

Figure 2: Faba bean samples with typical symptoms: top yellowing of leaves, leaf rolling and dwarfed appearance of leaves and top part of the plant.

In Austria, nanovirus symptoms appeared first in $P$. sativum in early June 2016 and shortly after in faba bean. In mid-late June, nearly every $V$. faba crop showed typical symptoms of nanovirus infection. In many faba bean and pea crops infection caused significant yield losses (Fig. 3). Typical symptoms of stunted growth, chlorosis and poorly developed pods were also found in lentils and vetch ( $V$. sativa). Thirty-two samples of $L$. culinaris, P. sativum, V. faba and V. sativa from Burgenland, Styria and Upper and Lower Austria were tested for nanovirus infection using PCR primers designed by (Kumari et al., 2010). The samples consisted of leaves pooled from several symptomatic plants from each field. Twenty-seven samples were positive for nanovirus infection. Representative amplicons from faba beans, lentils, peas, and vetch were sequenced (KY191009 KY191023) and had 99.6 to $100 \%$ identity to PNYDV (KC979043).

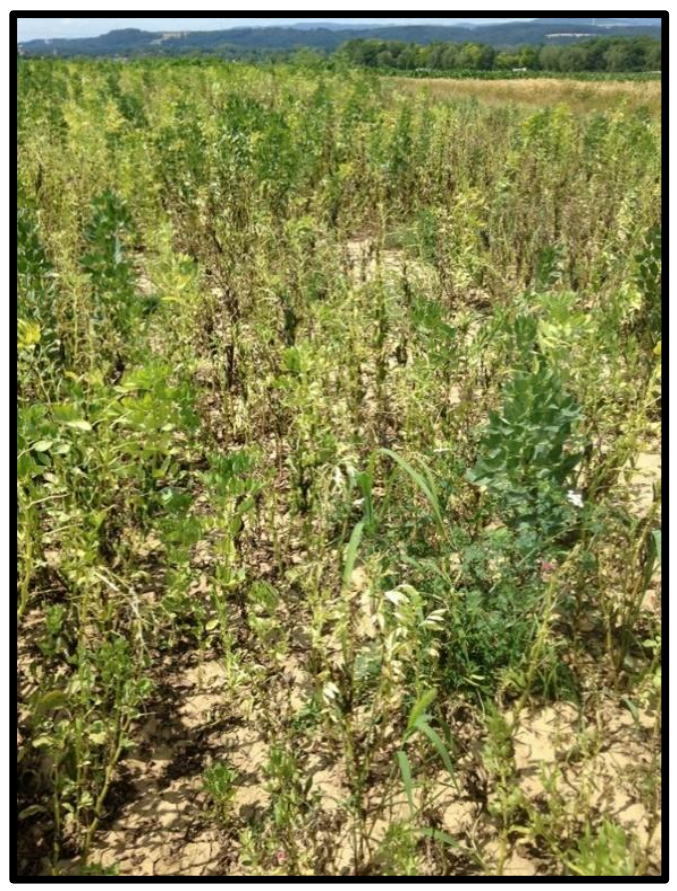

Figure 3: Faba bean crop heavily infected with nanoviruses. 
This is the first report of L. culinaris, V. faba and V. sativa as natural hosts of PNYDV in Austria and Germany. Due to changes in government policy, the area of legumes grown in Germany doubled from 2012 to 2015 (Table 1) with further increases expected. However, limited host range experiments on peas and faba beans have not identified PNYDV-resistant accessions in Austria or Germany suggesting that legume production in central Europe is threatened by PNYDV infection.

Table 1: Acreages (ha) of selected legumes grown in Germany and Austria in 2012 and 2015.

\begin{tabular}{|l|c|c|c|c|}
\hline \multirow{2}{*}{ Crop } & \multicolumn{2}{|c|}{ Germany $^{\text {a }}$} & \multicolumn{2}{c|}{ Austria $^{\text {b }}$} \\
\cline { 2 - 5 } & 2012 & $\mathbf{2 0 1 5}$ & $\mathbf{2 0 1 2}$ & $\mathbf{2 0 1 5}$ \\
\hline Glycine max & 5,000 & 11,000 & 36,955 & 56,867 \\
\hline Pisum sativum (protein peas only) & 44,800 & 79,100 & 10,700 & 7,183 \\
\hline Vicia faba & 15,800 & 37,700 & 6,854 & 10,822 \\
\hline Total & 65,600 & 127,700 & 54,509 & 74,872 \\
\hline
\end{tabular}

${ }^{a}$ Anonymous, 2016. Eiweisspflanzenstrategie.

http://www.bmel.de/DE/Landwirtschaft/Pflanzenbau/Ackerbau/_Texte/Eiweisspflanzen strategie.html, (Accessed 07 August 2016)

Burghardt B, Schaack D, Von Schenck W, 2016. AMI Markt Bilanz Getreide Ölsaaten Futtermittel 2016. In. Bonn: Agrarmarkt Informations-Gesellschaft mbH, 225 pages. www.AMI-informiert.de (Accessed 07.08.2016)

${ }^{b}$ Agrar Markt Austria, 2016. Flächenauswertung der Mehrfachanträge (MFA). In. Wien: Agrar Markt Austria. www.ama.at/Marktinformationen/Getreide-und-Olsaaten/AktuelleInformationen/2016/AMA-Flaechenauswertung-2016 (Accessed 07 August 2016) 


\subsection{First report of pea necrotic yellow dwarf virus in The Netherlands}

Yahya Zakaria Abdou Gaafar, T. Timchenko and H. Ziebell

This article has been published in a slightly modified version as:

Gaafar Y, Timchenko T, Ziebell H, 2017. First report of Pea necrotic yellow dwarf virus in The Netherlands. New Disease Reports 35, 23. doi: 10.5197/j.2044-0588.2017.035.023 
Pea necrotic yellow dwarf virus (PNYDV) is a nanovirus that was first detected in pea crops (Pisum sativum) in Saxony-Anhalt, Germany in 2009 (Grigoras et al., 2010a). In 2016, PNYDV was detected countrywide in both Germany and Austria not only on pea but also on faba bean (Vicia faba), vetch ( $V$. sativa) and lentil (Lens culinaris) causing severe yield losses (Gaafar et al., 2016).

During a routine survey of twelve green pea crops in the Province of Flevoland (The Netherlands), plants with virus-like symptoms were noticed (Fig. 1). Symptomatic plant material was pooled from each field and analysed by ELISA for typical pea viruses: Alfalfa mosaic virus, Cucumber mosaic virus, luteo-/poleroviruses, Pea enation mosaic virus (PEMV), potyviruses, and Red clover vein mosaic virus-like carlaviruses, and nanoviruses. PEMV was detected in all fields while luteo-/poleroviruses were found in one field. Two samples each from different pea fields reacted positively using a broad nanovirus monoclonal antibody mixture (Gaafar et al., 2016). The lack of reaction with a monoclonal antibody mixture designed to detect only Faba bean necrotic stunt virus and Faba bean necrotic yellows virus suggested infection with Pea necrotic yellow dwarf virus (PNYDV). This was confirmed by PCR using PNYDV specific primers targeting the eight PNYDV components producing bands of approximately $1 \mathrm{~kb}$ (Table 1). All PCR products were cloned using the NEB PCR cloning kit (New England Biolabs, Germany) and at least four clones for each component were sequenced in both directions. The sequences of the eight components of the two Dutch isolates (NL HZ16-186 and NL HZ16-189) had between 96.7 and $99.9 \%$ identity with the equivalent PNYDV components of an isolate from Germany and between 96.7 and $99.8 \%$ with an Austrian isolate (Table 1). The sequences of the Dutch PNYDV isolates have been deposited in GenBank (KY593279 - KY593294).

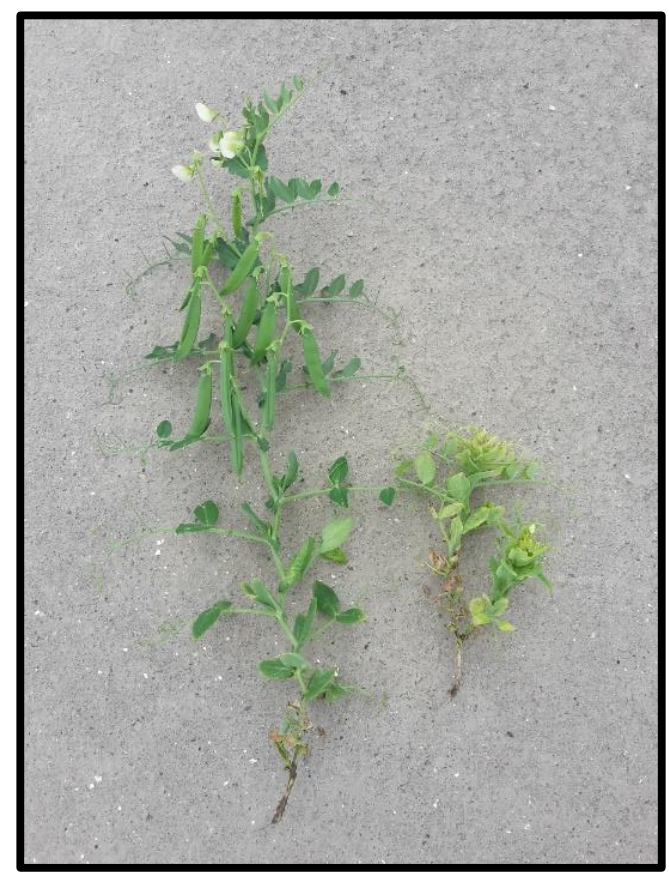

Figure 1: Non-infected (left) and virus-infected (right) pea plants. Typical Pea necrotic yellow dwarf virus symptoms included severe stunting and dwarfing of plants, yellowing and leaf-rolling. 
Table 1: List of the primers used for Pea necrotic yellow dwarf virus identification and pairwise comparisons between the sequences of the Dutch isolates (NL HZ16-186 and NL HZ16-189) and isolates from Austria (GenBank Accession No. KC979043 - KC979050) and Germany (GU553134 and JN133279 - JN133285).

\begin{tabular}{|c|c|c|c|c|c|c|}
\hline $\begin{array}{c}\text { PNYDV } \\
\text { component }\end{array}$ & Primer name & Primer sequence & \multicolumn{2}{|r|}{ References } & \multicolumn{2}{|c|}{ Identity \% } \\
\hline \multirow{2}{*}{ DNA-C } & priPeaCdir & 5' GCC GGA AGC TTG CCG GAC TGA CGG AG 3' & & КС979045 & 99.2 & 99 \\
\hline & priPeaCrev & 5' AGC TTC CGG CAA GAC GCA GTA ATT G 3' & & JN133280 & 99.5 & 99.1 \\
\hline \multirow{2}{*}{ DNA-M } & priPeaMdir & 5' TAC CTG AAC GTC CTG TAT CTT 3' & & KC979046 & 98.7 & 98.3 \\
\hline & priPeaMrev & $5^{\prime}$ TCA GGT ACT GAA TTA CTT GCC 3' & & JN133281 & 98.3 & 97.4 \\
\hline \multirow{2}{*}{ DNA-N } & priPeaNdir & 5’ GAA GAA CCC AGG AAG GTG TTG C $3^{\prime}$ & & KC979047 & 99.4 & 98.9 \\
\hline & priPeaNrev & 5' GGT TCT TCC AAT TTA СCT TTC ATG G 3' & & JN133282 & 99.9 & 99.2 \\
\hline \multirow{2}{*}{ DNA-R } & priPeaRdir & 5' GGA ATA CCA AGG TGA GTT ACG G 3' & 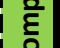 & KC979043 & 99.8 & 99.7 \\
\hline & priPeaRrev & 5' TAT TCC CTG AGA GTC CCG GAC 3' & & GU553134 & 99.8 & 99.5 \\
\hline \multirow{2}{*}{ DNA-S } & priPeaSdir & 5' AAC CTC CGG ATA TCA CCA GAT 3' & & KC979044 & 99.3 & 98.7 \\
\hline & priPeaSrev & 5' CCG GAG GTT TTA TIT CAA AAC CAA C 3' & . & JN133279 & 99.3 & 99.8 \\
\hline \multirow{2}{*}{ DNA-U1 } & priPeaU1dir & $5^{\prime}$ TGG TGA AGA AAT TGC AGG TGA T 3' & & KC979048 & 98 & 98.7 \\
\hline & priPeaU1rev & 5' TTC ACC AGT TTC TCG TCA GAA C 3' & & JN133283 & 98.3 & 98.8 \\
\hline \multirow{2}{*}{ DNA-U2 } & priPeaU2dir & 5' GAT CAA GAA CAA GGT TAG TTT ATG 3' & & KC979049 & 98.2 & 96.7 \\
\hline & priPeaU2rev & $5^{\prime}$ TCT TGA TCG GAG ACG AAC TGG A 3' & & JN133284 & 98.2 & 96.7 \\
\hline \multirow{2}{*}{ DNA-U4 } & priPeaU4dir & 5’ ATC AAG TCT GAA GAT GAT ACG 3' & & KC979050 & 99.1 & 99.3 \\
\hline & priPeaU4rev & 5' GAC TTG ATT TCA ACA TCT CTT TCA C 3' & & JN133285 & 99.8 & 99.7 \\
\hline
\end{tabular}

To our knowledge, this is the first report of PNYDV in The Netherlands. This indicates that nanoviruses are far more spread throughout Europe than previously thought (Grigoras et al., 2014). As PNYDV is aphid-transmitted in a circulative, nonpropagative manner, it is expected that more nanovirus diseases will occur in the future as changes in climatic conditions (especially milder winters in Central Europe) favour aphid survival thus facilitating the spread of these viruses (Ziebell, 2017). 


\subsection{Molecular characterisation of the first occurrence of pea necrotic yellow dwarf virus in Denmark}

Yahya Zakaria Abdou Gaafar, G. Cordsen Nielsen and H. Ziebell

This article has been published in a slightly modified version as:

Gaafar Y, Cordsen Nielsen G, Ziebell H, 2018. Molecular characterisation of the first occurrence of Pea necrotic yellow dwarf virus in Denmark. New Disease Reports 37, 16. doi: 10.5197/j.2044-0588.2018.037.016 
Pea necrotic yellow dwarf virus (PNYDV), a member of the Nanovirus genus, has been reported from numerous European countries causing yield loss in peas, faba beans, vetches and lentils (Grigoras et al., 2010a; Grigoras et al., 2014; Gaafar et al., 2016; Gaafar et al., 2017). In July and August 2016, five faba bean (Vicia faba) samples were received from five fields in different regions of Denmark, in which 2 to $25 \%$ plants were diseased. Affected plants displayed leaf-rolling, yellowing, and symptoms of severe stunting (Figs. 1-2).

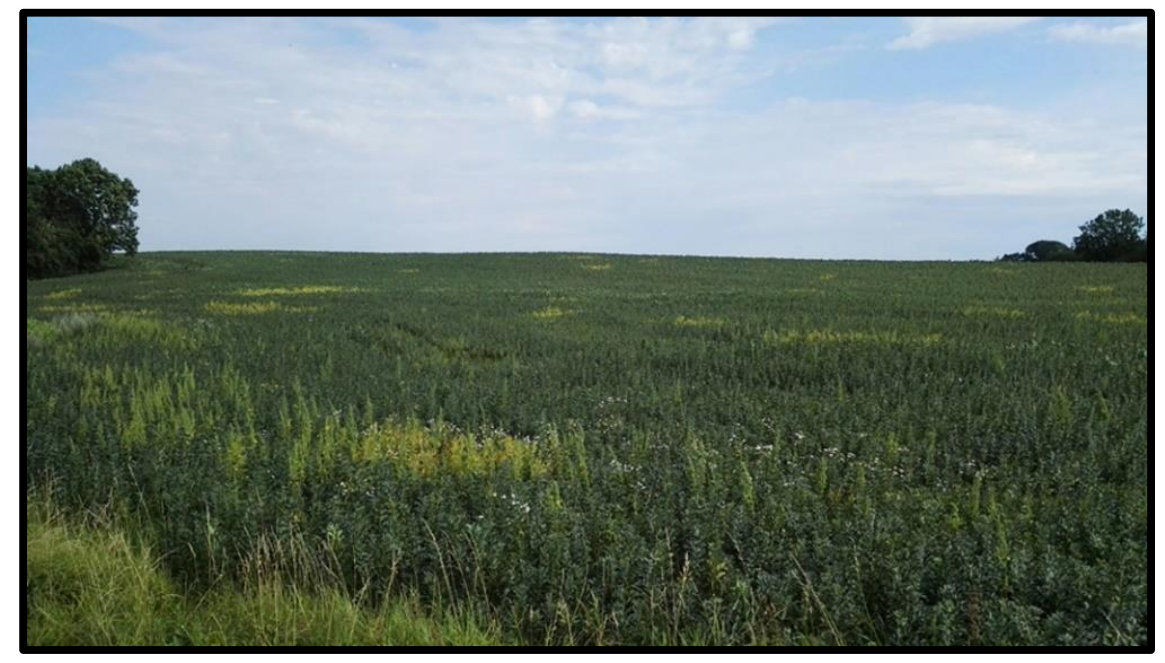

Figure 1: Symptoms of Pea necrotic yellow dwarf virus in a faba bean field near Åbenrå, Denmark (photograph courtesy Morten Steg).

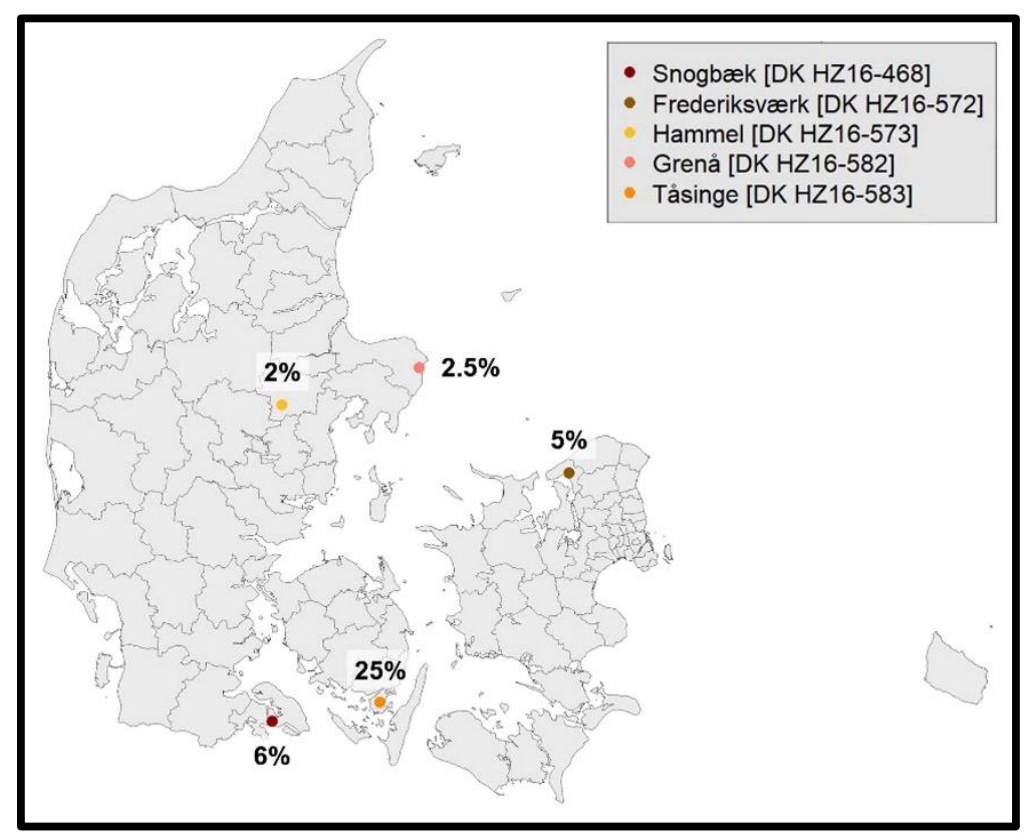

Figure 2: Map of Pea necrotic yellow dwarf virus-infected faba bean fields in Denmark in 2016. The coloured circles indicate the locations of the fields. The percentages represent the proportion of symptomatic plants within each field. The map was generated using MapDK (Barfort, 2015. mapDK: Maps of Denmark. R Package Version 0.3.0) and ggplot2 (Wickham, 2009. ggplot2: Elegant 
Graphics for Data Analysis. New York, USA: Springer-Verlag) on R 3.3.2 (R Project for Statistical Computing; https://www.r-project.org).

The samples reacted positively when analysed with ELISA using antibodies against PNYDV (Gaafar et al., 2016). DNA was extracted and PNYDV infection was confirmed by PCR using primer pairs specific for components R and S (Gaafar et al., 2017). Additionally, the full genomes of these Danish PNYDV isolates were determined. Rolling circle amplification was performed using a TempliPhiTM 100 Amplification Kit (GE Healthcare Limited, UK). Libraries were prepared from the products and submitted for highthroughput sequencing on two Illumina platforms, three samples on HiSeq $(2 * 150)$ and two on MiSeq $(2 * 301)$. The paired-end reads were analysed using Geneious 11.0.4 software (Biomatters Limited, NZ). The reads were trimmed, error corrected and normalised, then used for de novo assembly. Assembled contigs were compared with the GenBank non-redundant nucleotide and protein databases using BLASTn and BLASTx, respectively.

The results confirmed the presence of all eight PNYDV components in all samples. Additionally, an alphasatellite 3 sequence was found associated with the DK HZ16-572 and DK HZ16-582 isolates, and a satellite DNA-X sequence was found associated with DK HZ16572. The PNYDV genome Drohndorf-15 (Germany; GenBank Accession Nos. NC_023154 to NC_023161) was used to assemble the eight components of the Danish isolates and Austrian sequences (Gross-Enzernsdorf_1) for alphasatellite 3 and satellite DNA-X (KC979052 and KC979053, respectively) by mapping to reference. The sequences of the Danish PNYDV isolates can be accessed on GenBank (MH000227 to MH000269).

A neighbour-joining tree (Jukes-Cantor genetic distance model and 1000 bootstrap replicates) was created using Geneious Tree Builder (Fig. 3) and the alignment (ClustalW 2.1) of the concatenated genome components (DNA-R, $-\mathrm{S},-\mathrm{C},-\mathrm{M},-\mathrm{N},-\mathrm{U} 1,-\mathrm{U} 2$ and $-\mathrm{U} 4$ ) from the Danish PNYDV isolates as well as the Austrian, Dutch and German isolates. Additionally, a pairwise comparison between the associated alphasatellite 3 of DK HZ16572 and DK HZ16-582 showed 99\% nucleotide identity to each other, and $99.1 \%$ and 99.3\% identity when compared with the Gross-Enzersdorf_1 isolate, respectively. Finally, DNA-X of DK HZ16-572 shared $98.8 \%$ identity with Gross-Enzersdorf_1. The tree and the sequence alignments indicate that the Danish isolates are very closely related to other European isolates of PNYDV. 


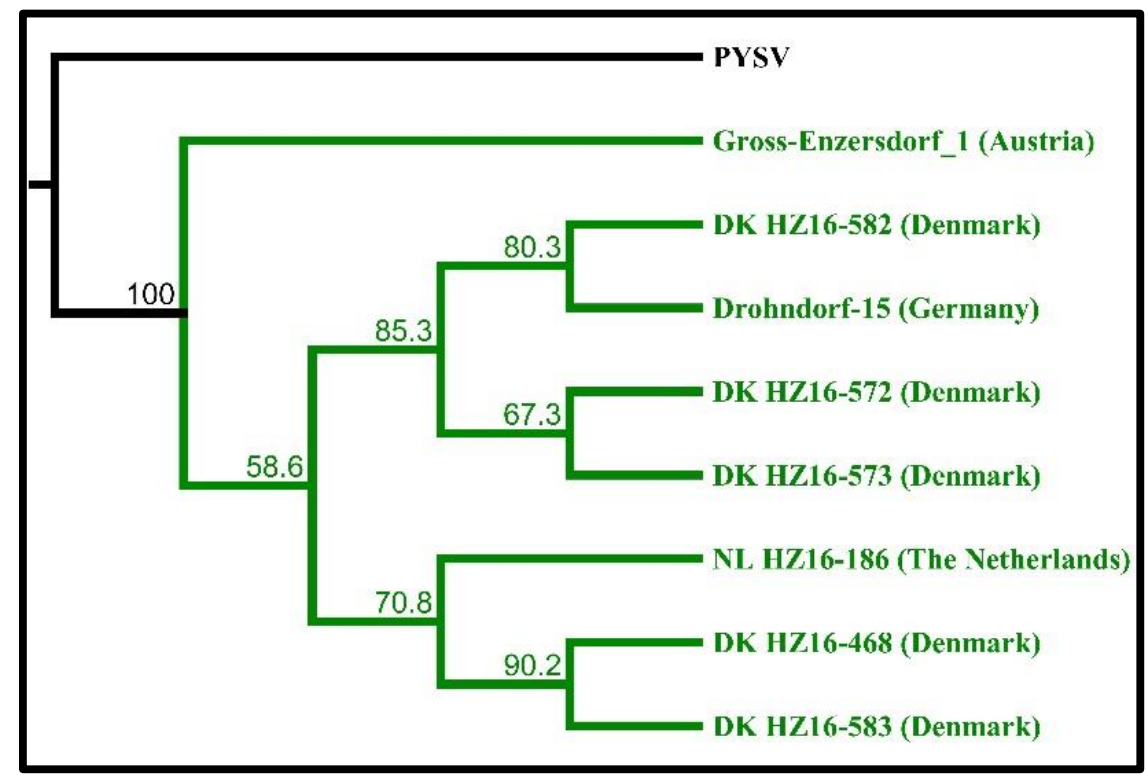

Figure 3: Phylogenetic neighbour-joining tree representing the relationship between the concatenated genome components (DNA-R, $-\mathrm{S},-\mathrm{C},-\mathrm{M},-\mathrm{N},-\mathrm{U} 1,-\mathrm{U} 2$ and $-\mathrm{U} 4$ ) of Danish Pea necrotic yellow dwarf virus isolates and those from Austria (KC979043 - KC979050), Germany (NC_023154NC_023161) and The Netherlands (KY593279 - KY593286). Pea yellow stunt virus (PYSV) (NC_023296 - NC_023298, NC_023303 and NC_023308 - NC_023311) was used as an outgroup.

To our knowledge, this is the first report of a nanovirus disease in Denmark demonstrating that PNYDV is also widespread throughout Denmark. This is further evidence that PNYDV (and possible other nanoviruses) is an emerging threat for legumes not only in Denmark which has an increasing area of legume production (Table 1) but also in other European countries.

Table 1: Areas of cultivated legumes (hectares) in Denmark from 2014 to 2017 (Statistics Denmark, 2017. https://www.statbank.dk/. Accessed on 7-3-2018).

\begin{tabular}{|l|c|c|c|c|}
\hline Year & $\mathbf{2 0 1 4}$ & $\mathbf{2 0 1 5}$ & $\mathbf{2 0 1 6}$ & $\mathbf{2 0 1 7}$ \\
\hline Total legumes & 8,400 & 11,900 & 15,700 & 20,600 \\
\hline Faba bean & 3,900 & 6,900 & 10,700 & 14,800 \\
\hline Pea & 4,200 & 4,700 & 4,800 & 5,400 \\
\hline
\end{tabular}


Chapter 3: Investigating the pea virome in Germany - old friends and new players in the field(s)

Yahya Zakaria Abdou Gaafar, K. Herz, J. Hartrick, J. Fletcher, A. Blouin, R. MacDiarmid and H. Ziebell 


\subsection{Abstract}

Peas are important legumes for human and animal consumption and are also being used as green manure or intermediate crops to sustain and improve soil condition. Pea production faces different constraints by fungi, bacteria pests and viral diseases. We investigated the virome of German pea crops over the course of three successive seasons in different regions of pea production in order to get an overview of the existing viruses. Pools from 540 plants randomly selected from symptomatic and asymptomatic pea, and non-crop plants surrounding the pea fields were used for ribosomal RNAdepleted total RNA extraction followed by high-throughput sequencing and RT-PCR confirmation. Thirty-five different viruses were detected in addition to eight associated nucleic acids. From these viruses, 25 are classified as either new viruses, novel strains or viruses that have not been reported previously from Germany. Pea enation mosaic virus (PEMV) was the most prevalent virus detected in the pea crops followed by turnip yellows virus (TuYV) which was also found in the surrounding non-legume weeds. Moreover, a new emaravirus was detected in peas in one region for two successive seasons. The results revealed a high virodiversity in the German pea fields that poses new challenges to diagnosticians, researchers, risk assessors and policy makers as the impact of the new findings are currently unknown.

\subsection{Introduction}

Green peas (Pisum sativum L.) are popular vegetables in Germany. The production of green peas increased from 4,444 ha in 2010 to 5,488 ha in 2018 (Behr, $2015,2019)$. In addition, due to the "Protein Strategy" of the Federal Government of Germany, the production areas of protein peas used as animal fodder, green manure or as intermittent crops, increased from 57,200 ha in 2010 to 70,700 ha in 2018 (BMEL, 2019). However, depending on the intended use of the crop, pea production in Germany is highly regionalised. The main green pea production areas are located in Saxony due to the nearby frozen foods processing facilities. Seed production of peas is predominantly carried out in Saxony-Anhalt. By contrast, green pea production for the fresh market or protein pea production for animal fodder/green manure are scattered around the country often associated with small scale or organic farming.

Pea plants are known to be hosts to several viruses from different families e.g. Luteoviridae, Nanoviridae and Potyviridae, that often occur in mixed infections (Musil, 1966; Kraft, 2008; Bos et al., 1988; Gaafar et al., 2016; Gaafar \& Ziebell, 2019b). Due to the emergence of novel pea-infected viruses such as pea necrotic yellow dwarf virus and their subsequent detection across Germany and within neighbouring countries (Grigoras et al., 2010a; Gaafar et al., 2016; Gaafar et al., 2017; Gaafar et al., 2018a), we were interested to know whether more unknown and or previously undetected viruses were present in this high value crop growing in Germany. 
Conventional virus diagnostics generally depend on serological or molecular methods based on prior knowledge of the target virus. These specific tests do not address the potential presence of other viruses that may be present or contribute to the aetiology of a disease. In recent years, high-throughput sequencing (HTS) enabled identification of numerous new viruses from domesticated and wild plants (Roossinck et al., 2015; Gaafar et al., 2019f; Gaafar et al., 2019e). HTS allows sequencing of all the genetic material in a given sample, therefore there is no need for prior knowledge of the infectious agent (Adams et al., 2009; Roossinck et al., 2015; Maree et al., 2018). Improvements of HTS technologies and bioinformatic tools have helped to identify the virus community or virome of several crops (Coetzee et al., 2010; Czotter et al., 2018; Jo et al., 2018b) The generated data permits the description of plant virus biodiversity, discovery of new viruses and viroids, identification of genomic variants of the viral species and aids the development of specific and sensitive diagnostics (Coetzee et al., 2010; Gaafar et al., 2019c; Li et al., 2012; Gaafar et al., 2019d). However, the vast number of new viruses identified by HTS results in challenges for diagnosticians, pest risk assessors and policy makers as the risk to crop plants and alternative hosts by these new viruses need to be evaluated and diagnostic protocols developed and validated or adapted to these findings (MacDiarmid et al., 2013; Massart et al., 2017; Rott et al., 2017; Maree et al., 2018). Nevertheless, metagenomics data and biological studies will help us understand the viral ecology and evolution as well as epidemiology.

In this study, we were interested in the spatio-temporal description and changes of the pea virome in selected German regions with different pea production aims (fresh produce, frozen produce, seed production, etc.) over a period of three years. Furthermore, we investigated potential alternative virus reservoirs in terms of legume and non-legume groundcover that were associated with the production sites. To our knowledge this is the first metagenomics study of a crop plant that takes spatio-temporal changes into consideration.

\subsection{Material and Methods}

\subsubsection{Sampling}

Six green pea production regions in Germany were chosen for sampling as they included sites for human consumption, protein pea production sites, seed production sites as well as experimental trial sites. The regions named by their county designation were Salzlandkreis-1: pea seed production, Salzlandkreis-2: trial site heritage material, Münster: trial site pea breeding, Kreis Stormarn: protein pea production, Landkreis Rostock: trial site (green manure mixtures) and Landkreis Meißen: green pea production frozen produce for human consumption.

In each region, one typical production field was chosen randomly. Over three successive seasons (2016, 2017 and 2018 between June and July; Figure 1) these regions were sampled. From each field, ten symptomatic (showing virus-like symptoms) pea 
plants (SP) and ten asymptomatic pea plants (aSP), five surrounding non-crop legume plants $(s L)$ and five non-legume plants $(s n L)$ were collected where possible. The metadata were recorded, i.e., the symptoms of each plant sampled, the region, and the average temperature of the season for each region (Supplementary Table S1). Any deviations from the sampling strategy (i.e., in cases where no non-symptomatic peas or no surrounding legume plants could be detected) were also noted in (Supplementary Table S1).

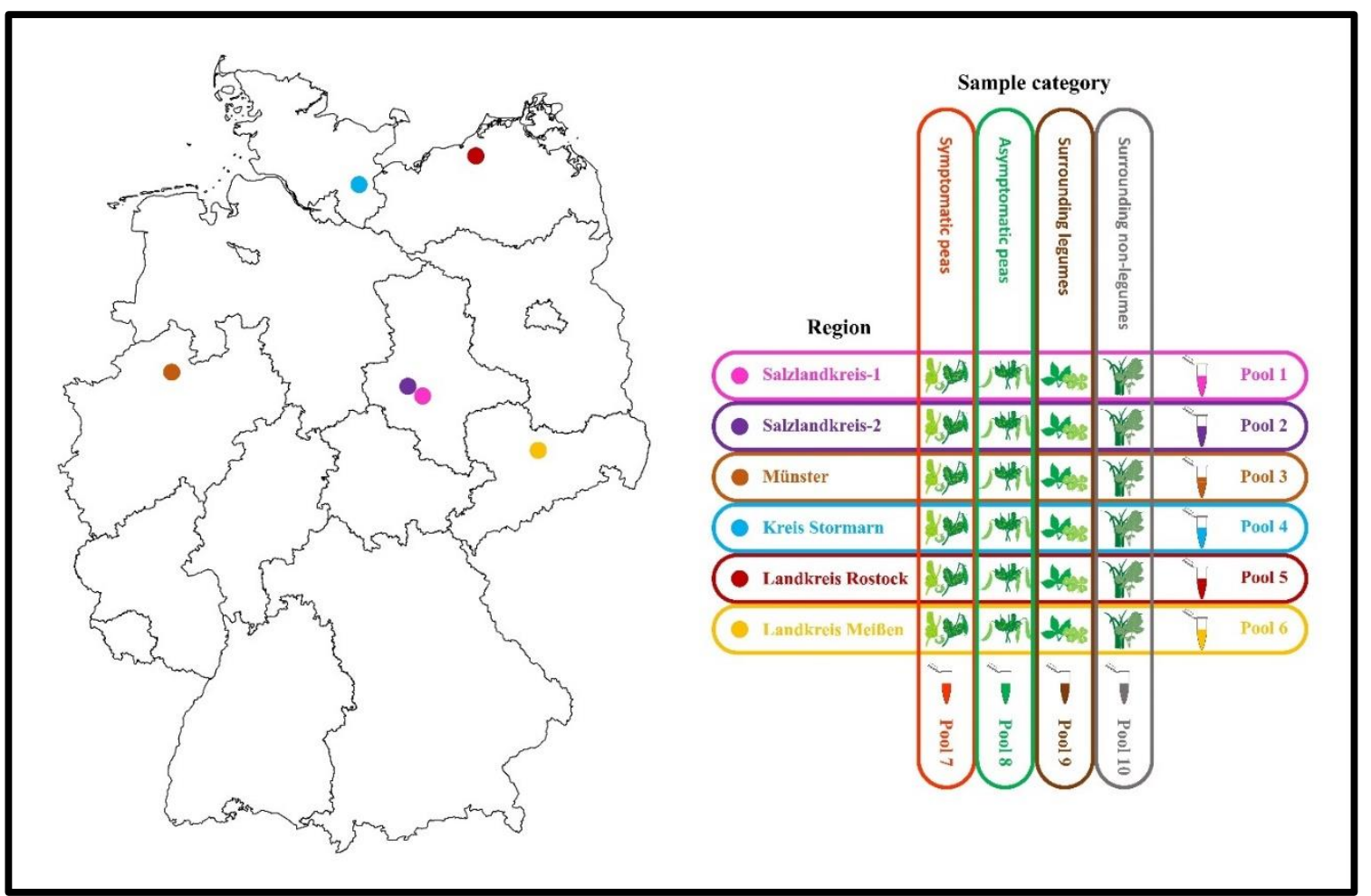

Figure 1: Map of the six different pea crop sampling regions in Germany and the sample pooling strategy used for each of three successive seasons 2016, 2017 and 2018.

Ten sample pools were prepared for each season i.e., six separate pools containing material from each of the six regions and four separate pools comprising material from all SP, aSP, SL or SnL samples, respectively, from all regions (Figure 1). From each plant sample, $100 \mathrm{mg}$ of fresh tissue was added to each pool. The samples were mixed and ground using a mortar and pestle under liquid nitrogen then collected into $50 \mathrm{ml}$ Falcon tubes. The pools were stored at $-20^{\circ} \mathrm{C}$ until RNA extraction. Additionally, the remaining material of fresh plant samples were stored at $-20^{\circ} \mathrm{C}$ for further analysis. 


\subsubsection{RNA extraction and high throughput sequencing}

Total RNA was extracted using innuPREP Plant RNA Kit (Analytik Jena) from a subsample of $300 \mu \mathrm{g}$ from each pool. Ribosomal RNA (rRNA) was depleted using RiboMinus ${ }^{\text {TM }}$ Plant Kit for RNA-Seq (Invitrogen). cDNA was synthesized using ProtoScript II Reverse Transcriptase (NEB) and random octanucleotide primers (8N), followed by second strand synthesis using NEBNext Ultra II Non-Directional RNA Second Strand Synthesis Module (NEB). The libraries were prepared from the double-stranded cDNA using Nextera XT Library Prep Kit (Illumina). The sequencing was performed on an Illumina MiSeq platform (301 x 2).

\subsubsection{Bioinformatic analysis}

Bioinformatic analysis was performed using Geneious Prime software (version 2019.1.1). The reads were quality trimmed and normalised. De-novo assembly was performed and the resulting contigs were searched against a local database of viruses and viroids sequences downloaded from NCBI using Blastn and Blastx (downloaded 13 August 2018). The generated consensus sequences were based on the highest quality threshold. Primers for virus validation were designed using a modified version of Primer3 (2.3.7) tool in Geneious Prime (Untergasser et al., 2012). Pairwise alignments were performed using Clustal W tool (v 2.1) in Geneious (Larkin et al., 2007). Neighbour joining phylogenetic trees were constructed using MEGA X software (Kumar et al., 2018b). The phylogenetic relationships were established according to the species demarcation criteria set by International Committee on Taxonomy of Viruses (ICTV), using the nucleotide sequences or the amino acid sequences of the capsid protein (CP) or the RNA dependent RNA polymerase (RdRP) for the respective families. The isolates were named by region number and season e.g., R1_16 stands for region one and the season 2016. The assembled virus sequences can be accessed in GenBank under accession nos.

(MN314973, MN399680-MN399748, MN412725-MN412751 and MN497793MN497846).

\subsubsection{RT-PCR confirmations}

For virus confirmation, total RNA was re-extracted from each pool as described above, followed by RT-PCR with the primers listed in Supplementary Table S2 and using the OneTaq One-Step RT-PCR Kit (NEB). The products were purified using Zymoclean Gel DNA Recovery Kit (Zymo Research) and Sanger sequenced using both RT-PCR primers.

\subsubsection{Statistical analysis}

Statistical analysis was performed using scripts written on $R$ (version 3.5.3) (R Core Team, 2019) The virus relative abundance (VRA) was calculated for each virus. VRA = the sum of the virus detections in each region in all season divided by the total of possible detection ( 3 seasons $\times 6$ regions $\times 4$ categories $=72$ ). The virus relative abundance was 0 for not detected to 1 for detected in all categories in all region in all seasons. Additionally, 
the virus incidence was calculated for the regions and the seasons. The Venn diagram was generated using Venny tool (Oliveros, 2015).

\subsection{Results}

\subsubsection{Collected metadata and HTS raw data}

General symptoms observed on peas were typical of PEMV such as translucent spots and leaf enations, stunting of top leaves, dwarfed plants, severe yellowing, mottling and leaf rolling. Interestingly, in 2016 it was not possible to find legumes in fields surrounding Salzlandkreis-1 and no asymptomatic peas were found in Salzlandkreis-2. Moreover, no surrounding legumes were collected in Landkreis Meißen 2018. Therefore, they were not included in the analyses. The details of the raw data generated from the HTS MiSeq platform are in Supplementary Table S3.

\subsubsection{Assignment of identified virus families}

A total of thirty-five viruses were detected by HTS and confirmed by RT-PCR in the different pools over the three seasons representing 14 different families in addition to several unassigned viruses (Figure $2 \mathrm{a}$ and $\mathrm{b}$ ). The family Luteoviridae was represented by seven species members, followed by the Secoviridae with six species members, then the Potyviridae with five members. During the three seasons, the highest virus diversity was in the SP pools with sixteen different virus species present, while the sL contained twelve different virus species. The snL pools included eleven virus species and the aSP had seven virus species (Figure $2 \mathrm{a}$ and $\mathrm{b}$ ). 

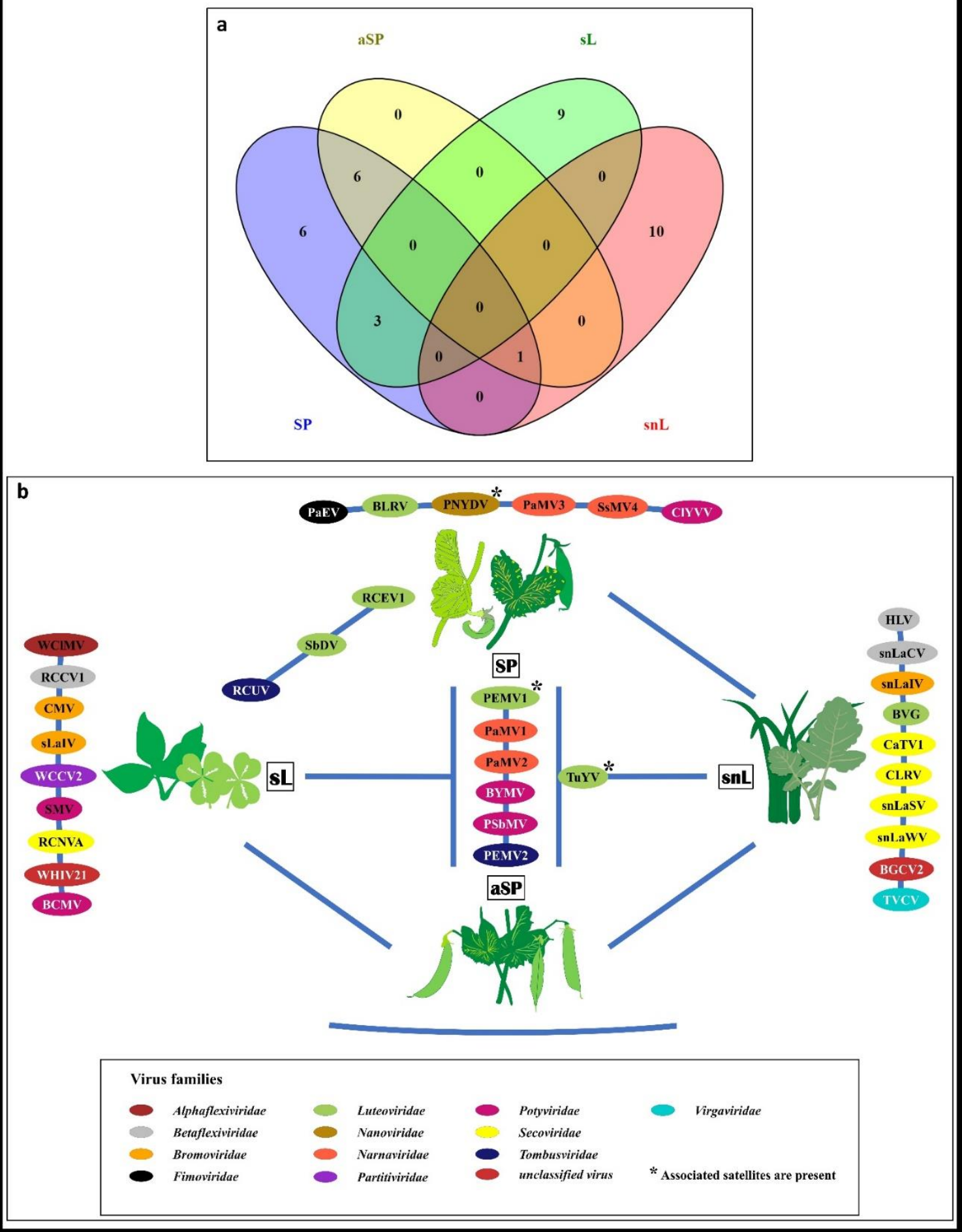

Figure 2: The virus species found in the four sampling categories symptomatic peas (SP), asymptomatic peas (aSP), surrounding legumes ( $s L$ ) and surrounding non-legumes (snL). Samples were collected in six pea growing regions in Germany over three successive seasons 2016, 2017 and 2018. a) Venn diagram representing the number of virus species to each category and shared between the four categories. b) network illustration of virus species of each category and shared viruses between them. Background colours of virus acronyms correspond to the background colours of the respective virus family. The virus names are: BVG: barley virus G, BCMV: bean common mosaic virus, BLRV: bean leafroll virus, BYMV: bean yellow mosaic virus, BGCV2: black grass cryptic virus 2, CaTV1: carrot torradovirus 1, CLRV: cherry leaf roll virus, CIYVV: clover 
yellow vein virus, CMV: cucumber mosaic virus, HLV: Heracleum latent virus, PaMV1: pea associated mitovirus 1, PaMV2: pea associated mitovirus 2, PaMV3: pea associated mitovirus 3, PaEV: pea associatted emaravirus, PEMV1: pea enation mosaic virus 1, PEMV2: pea enation mosaic virus 2, PNYDV: pea necrotic yellow dwarf virus, PSbMV: pea seed-borne mosaic virus, RCCV1: red clover carlavirus 1, RCEV1: red clover enamovirus 1, RCNVA: red clover nepovirus a, RCUV: red clover umbravirus, SSMV4: Sclerotinia sclerotiorum mitovirus 4, sLalV: SL associated ilarvirus, snLaCV: snL associated chordovirus, snLalV: snL associated ilarvirus, snLaSV: snL associated secoviridae, snLaWV: SnL associated waikavirus, SbDV: soybean dwarf virus, SMV: soybean mosaic virus, TVCV: turnip vein-clearing virus, TuYV: turnip yellows virus, WCCV2: white clover cryptic virus 2, WCIMV: white clover mosaic virus, WHIV21: Wuhan insect virus 21.

All identified viruses, the regions in which they were found and the pools from which they were identified are listed in Supplementary Table S4. The virus relative abundance (VRA) was calculated for each virus (Supplementary Table S4), showing that turnip yellows virus (TuYV) was the most abundant virus with a score of 0.472 followed by pea enation mosaic virus 2 (PEMV2) with a score of 0.444 and PEMV1 scoring 0.417 . Pea necrotic yellow dwarf virus (PNYDV) was the fourth most abundant virus in the study with a score of 0.153 and pea seed borne mosaic virus (PSbMV) was the fifth most abundant one with 0.139 .

Out of the viral reads identified from HTS, Figure 3 shows the percentage of each virus family in each region pool during the three seasons. Overall, the read percentage of the luteovirids was the highest, mainly because of high percentage of reads assigned to PEMV1 and TuYV. The second highest percentage was for the tombusvirids, due to the presence of PEMV2 reads, followed by the potyvirids, mainly because of PSbMV. 


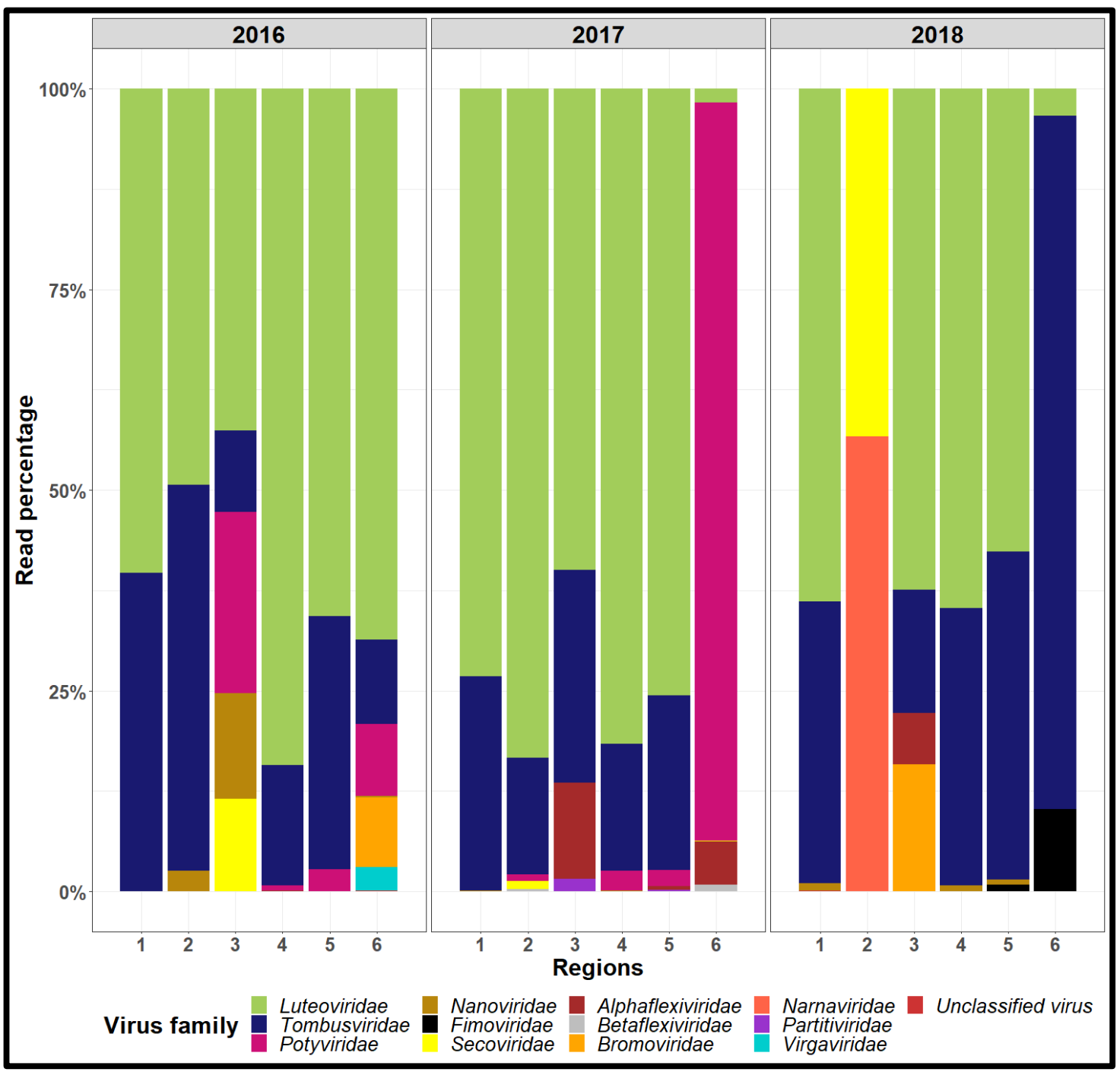

Figure 3: The read percentage of the different virus families identified in six different German pea growing regions over the three seasons 2016, 2017 and 2018.

\subsubsection{Detailed description of the viruses detected in German pea fields}

\subsubsection{Viruses of the Luteoviridae family}

Pea enation mosaic virus-1 (PEMV1) is a member of the genus: Enamovirus, family: Luteoviridae. PEMV1 was detected in all fields in almost all the three seasons in SP and aSP. The complete coding sequences of the PEMV1 isolates from each of the positive pools were assembled. The amino acid (aa) sequences of the RNA dependant RNA polymerases (RdRp) (used for taxonomic classification of luteovirids) shared between $96.9 \%$ to $98.4 \%$ aa identity with the RdRp of PEMV1 (NC_003629). They shared between $99.6 \%$ and $99.4 \%$ aa identity to each other.

In Münster 2016, a new strain of red clover enamovirus 1 (RCEV1) was identified in the sL samples. Its $\mathrm{RdRp}$ aa sequence showed $86.6 \%$ identity to a Czech strain (MG596229). Another isolate was detected in Landkreis Meißen in 2017, with 87.6\% aa identity to the other German strain and $95.3 \%$ to the Czech strain. The viruses grouped together within the Enamovirus genus clade (Figure 4). 


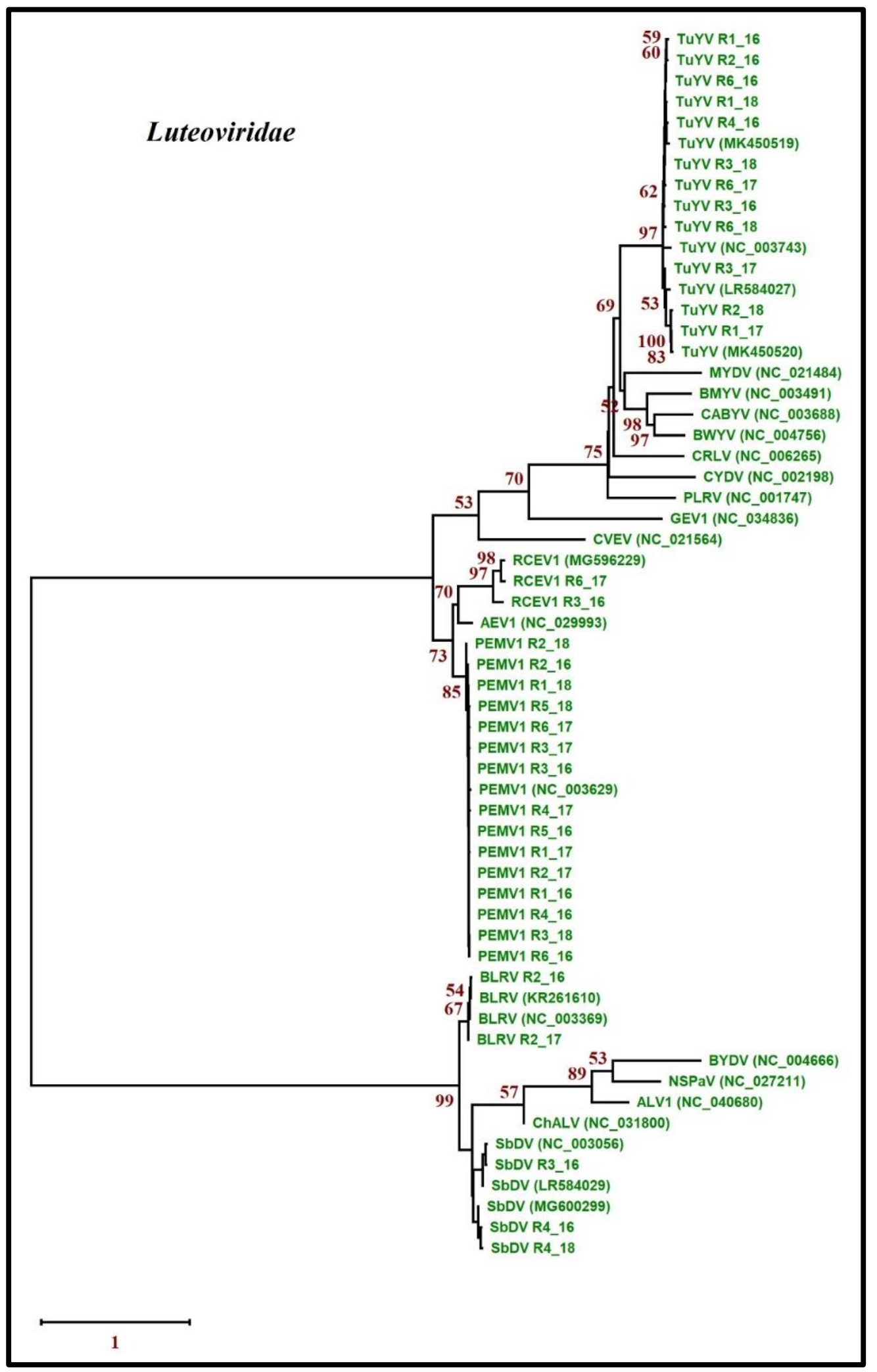

Figure 4: Neighbour joining trees (NJ) of virus isolates from the Luteoviridae family detected in German pea fields. The phylogenetic tree is based on amino acid sequence alignments of the readthrough RdRps and includes representatives of other viruses from the family Luteoviridae. Amino acid sequences were aligned with Clustal $W$ and NJ trees constructed with MEGA X. The percentage of the bootstrap values above $50 \%$ (1,000 replications) are shown at the nodes. The names of the viruses are as follow: AEV1: alfalfa enamovirus 1, ALV1: apple luteovirus 1, BLRV: 
bean leafroll virus, BMYV: beet mild yellowing virus, BYDV: barley yellow dwarf virus, BWYV: beet western yellows virus, CABYV: cucurbit aphid-borne yellows virus, ChALV: cherry associated luteovirus, CRLV: carrot red leaf virus, CVEV: citrus vein enation virus, CYDV: cereal yellow dwarf virus, GEV1: grapevine enamovirus-1, MYDV: maize yellow dwarf virus, NSPaV: nectarine stem pitting-associated virus, PEMV1: pea enation mosaic virus 1, PLRV: potato leafroll virus, RCEV1: red clover enamovirus $1, \mathrm{SbDV}$ : soybean dwarf virus and TuYV: turnip yellows virus. The Genbank accession numbers are mentioned in brackets.

Bean leafroll virus (BLRV) was detected in Salzlandkreis-2 for two successive seasons (2016 and 2017) in SP in addition to the SL pool in 2017. The isolates of BLRV were closely related to strain Manfredi from Argentina (KR261610); the RdRp aa sequences of the two isolates shared $99.1 \%$ identity to each other and $99.7 \%$ to the Argentinian strain.

Turnip yellows virus (TUYV) was detected in SP, aSP and snL pools over the three seasons. Pairwise aa alignments for the RdRp of the TuYV isolates showed that eight isolates were $95.6 \%$ to $97.1 \%$ identical to TuYV isolate JKI 29345 (MK450519). However, two isolates from Salzlandkreis-2 and Salzlandkreis-1 showed higher identities with $98.8 \%$ and $99.1 \%$ to isolate JKI 29344 (MK450520). Moreover, the isolate from Münster 2017 was closely related to TuYV (LR584027) with $95.3 \%$ aa identity.

Soybean dwarf virus (SbDV) was detected in peas and surrounding legumes in two regions i.e., Münster and Kreis Stormarn. The Kreis Stormarn SbDV RdRp aa sequences found in the survey were $98 \%$ aa identical to each other and shared closest aa identity to SDV-HZ1 isolate from the Czech Republic (MG600299) with $98.9 \%$ and $99.1 \%$ identity. In contrast, the isolate from Münster showed the closest identity to a SbDV isolate (LR584029) from Australia with $99.1 \%$ and only shared $89.8 \%$ and $90.2 \%$ identities to the isolates from Kreis Stormarn.

In addition, PEMV satellite RNAs (PEMVSatRNAs), TuYV associated RNAs (TuYVaRNAs) and a potential new associated RNA (TuYVaRNA2) were detected. The PEMV satellite RNA isolates had $95.6 \%$ to $97.9 \%$ nt identity to PEMVSatRNA (NC_003854, from the USA) and between $95.5 \%$ and $98.7 \%$ to each other. The TuYVaRNA isolates were $97.8 \%$ to $98.2 \%$ nt identical to TuYVaRNA isolate JKI 29345 (MK450521). The TuYVaRNA2 isolates were closely related to cucurbit aphid borne virus associated RNA (CABYVaRNA; KM486094, from the USA) with $79.8 \%$ to $81.9 \%$ nt identity.

\subsubsection{Tombusviridae (subfamily: Calvusvirinae)}

PEMV2 was associated with PEMV1 in almost all fields during the three seasons. The aa sequences of the RdRp of the PEMV2 isolates grouped together in a clade within the genus Umbravirus (Figure 4b). They showed $97.1 \%$ to $99.7 \%$ identity to each other and $93.3 \%$ to $94.1 \%$ identity to PEMV2 isolate (NC_003853) from USA. A new strain of red clover umbravirus (RCUV), a novel umbravirus found in red clover from the Czech Republic (Koloniuk, pers. comm.), was detected in the SL in Münster 2016 and the SP in Landkreis 
Meißen 2017. The complete coding sequence (CDS) shared $87.1 \% \mathrm{nt}$ identity and the aa sequence of the RdRp shared to $91.6 \%$ aa identity to this Czech isolate (MG596234).

\subsubsection{Potyviridae}

Five potyviruses were present in the pea fields as well as in surrounding leguminous weeds. Bean common mosaic virus (BCMV) was identified in Salzlandkreis-2 in 2017. The isolate shared $98.9 \%$ nt identity with to BCMV strain NL1 from The Netherlands (AY112735). Bean yellow mosaic virus (BYMV) was identified in Salzlandkreis2 and Salzlandkreis-1 in 2018. The isolates shared $83.9 \%$ aa identity to each other and 89.4\% to BYMV strain MB4 (NC_003492) from Japan. Clover yellow vein virus (CIYVV) was identified in Salzlandkreis-1 and Münster in 2018 in SP. Their polyproteins shared 95\% aa identity to each other and $95.5 \%$ and 93\%, respectively, to CIYVV strain No.30 (NC_003536, probably from Japan). Pea seed-borne mosaic virus (PSbMV) was found in two regions in Germany i.e., Salzlandkreis-2 and Kreis Stormarn in all three seasons of the study. The virus was detected in SP and in aSP, respectively. The polyproteins of the isolates had $97.7 \%$ to $100 \%$ aa identity to each other and had $98 \%$ to $99.7 \%$ aa identity to PSbMV strain DPD1 (NC_001671) from Denmark. Another potyvirus, soybean mosaic virus (SMV), could be detected in Salzlandkreis-2 in 2017. The Salzlandkreis-2 isolate of SMV was very closely related to strain G4 from South Korea with 99.5\% aa identity (FJ640979). Figure 5 shows a neighbour-joining tree representing the relationship between the polyproteins of the different potyviruses identified in the survey.

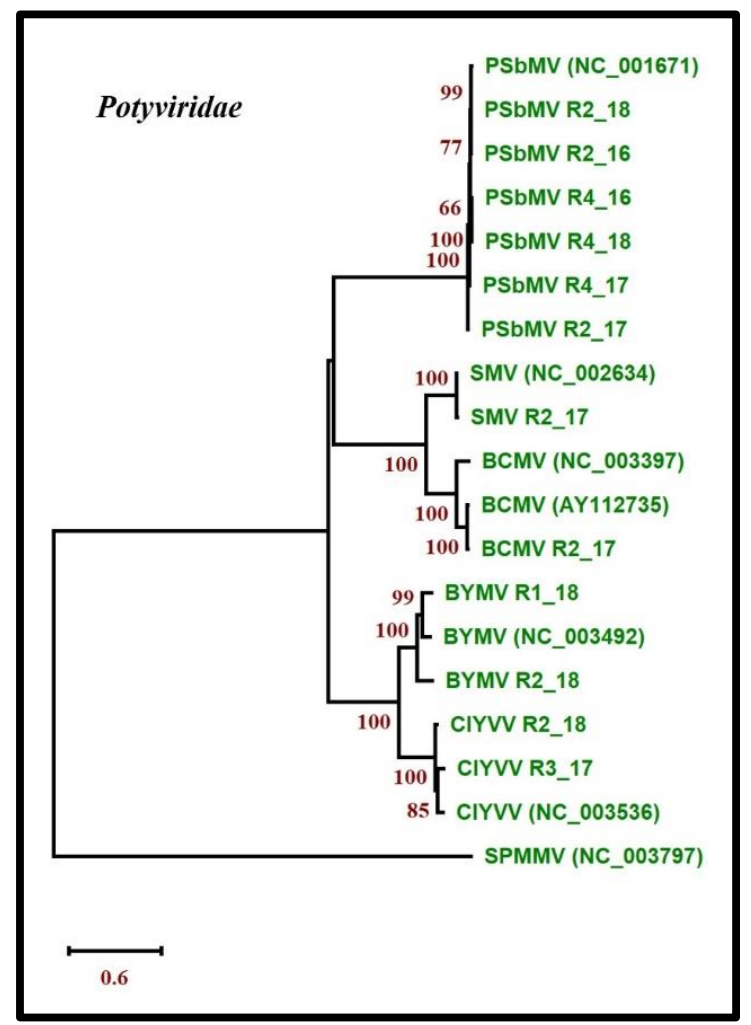

Figure 5: Neighbour joining trees (NJ) of Potyviridae virus isolates detected in German peas. The phylogenetic trees are based on amino acid sequence alignments of the polyproteins of the 
potyviruses found in this survey and includes representative species from the family Potyviridae. Amino acid sequences were aligned using Clustal W and NJ trees were constructed with MEGA X. The percentage of the bootstrap values above $50 \%$ (1,000 replications) are shown at the nodes. The names of the viruses are as follow: BCMV: bean common mosaic virus, BYMV: bean yellow mosaic virus, CIYVV: clover yellow vein virus, PSbMV: pea seed-borne mosaic virus, SMV: soybean mosaic virus and SPMMV: sweet potato mild mottle virus. The Genbank accession numbers are mentioned in the brackets.

\subsubsection{Nanoviridae: Pea necrotic yellow dwarf virus and its associated alphasatellites}

Pea necrotic yellow dwarf virus (PNYDV) was only detected in symptomatic peas. There was no indication of the presence of other nanoviruses such as black medic leafroll virus or pea yellow stunt virus (include Grigoras et al. 2016 here). All DNA-S components shared between 97 and $99.9 \%$ nt identities with the on NCBI available PNYDV DNA-S sequences (originating from Germany and Austria). Additionally, six PNYDV alphasatellites (PNYDVaSat) were identified in the survey i.e., alphasatellites 1 and 3, and four new alphasatellites, tentatively named PNYDV-associated alphasatellites 4, 5, 6 and 7.

The PNYDV $\alpha$ Sat1 isolate shared $99.6 \%$ nt identity with PNYDV $\alpha$ Sat1 isolate GrossEnzersdorf_1 (accession no. NC_038958) from Austria. The three isolates of PNYDVaSat3 shared between $99.3 \%$ and $99.4 \%$ nt identities to PNYDVaSat3 isolates GrossEnzersdorf_1 (Austria) and DK HZ16-582 from Denmark (NC_038959 and MH000253, respectively). The isolates of PNYDV $\alpha$ Sat4, PNYDV $\alpha$ Sat5, PNYDV $\alpha$ Sat6 and PNYDV $\alpha$ Sat7 were 1,030 nt, $991 \mathrm{nt}, 1,037 \mathrm{nt}$ and 1,015 nt in length, respectively. PNYDVaSat4 showed closest nt identities to faba bean necrotic yellows virus C7 alphasatellite (FBNYC7 $\alpha$ Sat; NC_003565) from Egypt and milk vetch dwarf virus C10 alphasatellite (MVDVC10 $\alpha$ Sat; NC_003647) from Japan with $82.3 \%$ to $82.9 \%$. PNYDVaSat5 isolates shared the highest identities with $81.1 \%$ to $81.3 \%$ with parsley severe stunt virus alphasatellite 1 (PSSVaSat1; MK039138, from Germany). PNYDVaSat6 isolates had $82.8 \%$ identity to cow vetch latent virus alphasatellite (CVLVaSat; MF535455) from France and the PNYDV $\alpha$ Sat7 isolate had $84.4 \%$ identity to the same CVLVaSat isolate. The phylogenetic relationships of those alphasatellites are shown in Figure $6 a$ and $b$. 


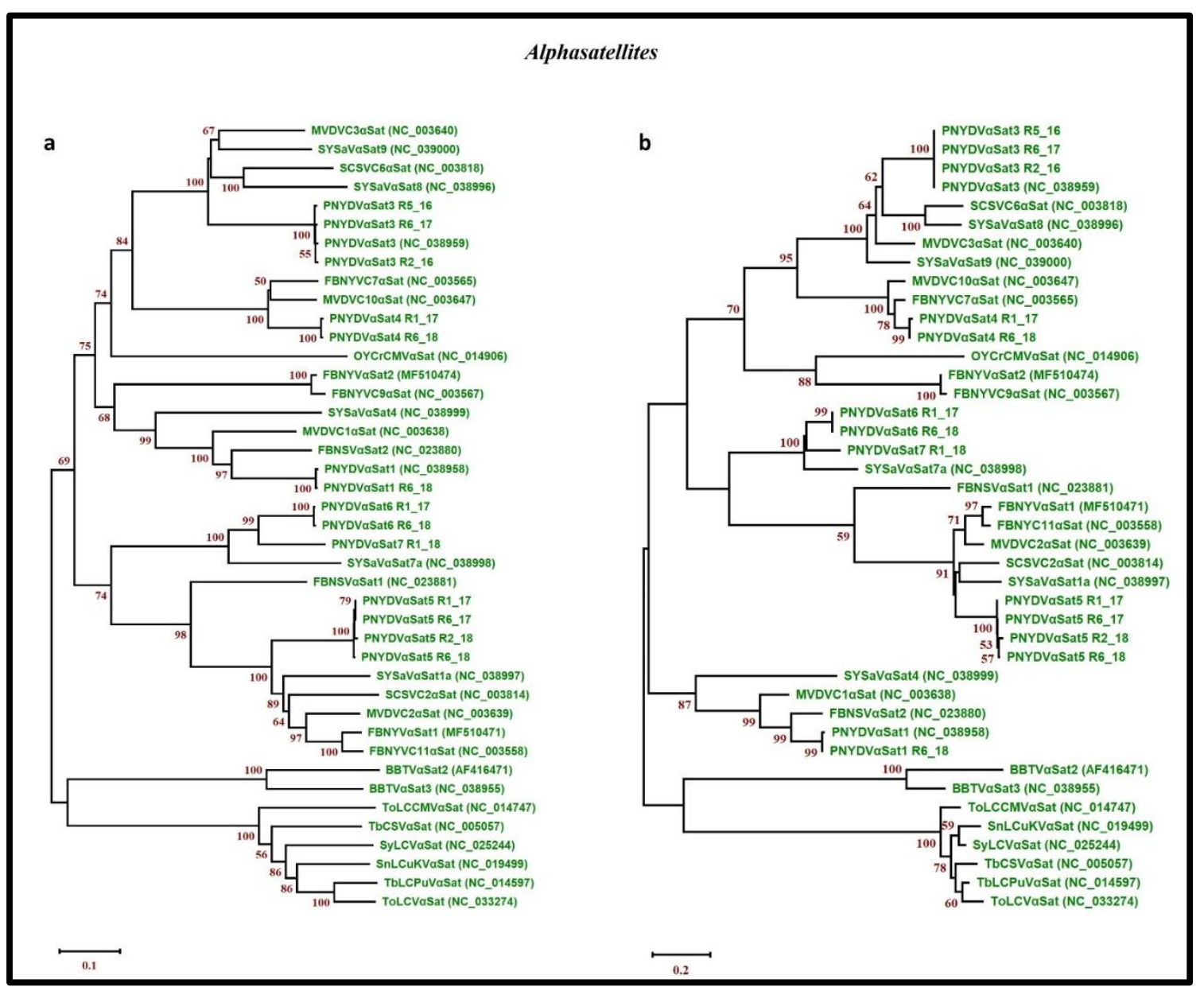

Figure 6: Neighbour joining trees (NJ) of PNYDV alphasatellites (PNYDVaSat) detected in German peas. The phylogenetic trees are based alignments of: (a) the nucleotide sequences (b) the amino acid sequences of the alphasatellite sequences identified in this study and include selected representatives of other alphasatellites. The sequences were aligned with Clustal $\mathrm{W}$ and $\mathrm{NJ}$ trees constructed with MEGA X. The percentage of the bootstrap values above $50 \%(1,000$ replications) are shown at the nodes. The names of the alphasatellites are as follow: BBTV $\alpha$ Sat2: banana bunchy top virus alphasatellite 2, BBTV $\alpha$ Sat3: banana bunchy top virus alphasatellite 3, FBNSV $\alpha$ Sat1: faba bean necrotic stunt virus alphasatellite 1, FBNSV $\alpha$ Sat2: faba bean necrotic stunt virus alphasatellite 2, FBNYV $\alpha$ Sat1: faba bean necrotic yellows virus alphasatellite 1, FBNYV $\alpha$ Sat2: faba bean necrotic yellows virus alphasatellite 2, FBNYVC7 $\alpha$ Sat: faba bean necrotic yellows virus C7 alphasatellite, FBNYVC9 $\alpha$ Sat: faba bean necrotic yellows virus C9 alphasatellite, FBNYC11 $\alpha$ Sat: faba bean necrotic yellows virus C11 alphasatellite, MVDVC1aSat: milk vetch dwarf virus C1 alphasatellite, MVDVC2 $\alpha$ Sat: milk vetch dwarf virus C2 alphasatellite, MVDVC3 $\alpha$ Sat: milk vetch dwarf virus C3 alphasatellite, MVDVC10aSat: milk vetch dwarf virus C10 alphasatellite, OYCrCMV $\alpha$ Sat: Okra yellow crinkle Cameroon virus alphasatellite, PNYDV $\alpha$ Sat1: pea necrotic yellow dwarf virus alphasatellite 1, PNYDVaSat3: pea necrotic yellow dwarf virus alphasatellite 3, PNYDV $\alpha$ Sat4: pea necrotic yellow dwarf virus alphasatellite 4, PNYDVaSat5: pea necrotic yellow dwarf virus alphasatellite 5, PNYDVaSat6: pea necrotic yellow dwarf virus alphasatellite 6, PNYDVaSat7: pea necrotic yellow dwarf virus alphasatellite 7, SCSVC2 $\alpha$ Sat: subterranean clover stunt virus C2 alphasatellite, SCSVC6aSat: subterranean clover stunt virus C6 alphasatellite, SnLCUKV $\alpha$ Sat: alphasatellite, SyLCV $\alpha$ Sat: Synedrella leaf curl virus alphasatellite, SYSaV $\alpha$ Sat1a: Sophora yellow stunt associated virus alphasatellite 1a, SYSaVaSat4: Sophora yellow stunt associated virus alphasatellite 4, SYSaVaSat7a: Sophora yellow stunt associated virus 
alphasatellite 7a, SYSaVaSat8: Sophora yellow stunt associated virus alphasatellite 8, SYSaVaSat9: Sophora yellow stunt associated virus alphasatellite 9, TbCSV $\alpha$ Sat: tobacco curly shoot virus alphasatellite, TbLCPuV $\alpha$ Sat: tobacco leaf curl Pusa virus alphasatellite, ToLCCMVaSat: tomato leaf curl Cameroon virus alphasatellite and ToLCV $\alpha$ Sat: tomato leaf curl virus alphasatellite. The Genbank accession numbers are mentioned in the brackets.

\subsubsection{Fimoviridae: Pea associated emaravirus (New virus)}

A new emaravirus was identified in symptomatic peas in Landkreis Meißen for two successive season 2017 and 2018. The virus showed high similarity to other members of genus Emaravirus i.e., fig mosaic virus (FMV), pigeonpea sterility mosaic virus 2 (PPSMV2) and rose rosette virus (RRV). Members of the genus Emaravirus (family Fimoviridae, order Bunyavirales) have segmented, linear, single-stranded, negative-sense RNA genomes (Elbeaino et al., 2018). Their genomes are composed of up to 8 segments. The partial sequences of segments RNA1 to 6 were assembled from the two isolates discovered from the two seasons. An NJ tree of the nucleocapsid protein (NP) aa sequences of the two isolates was constructed, and those isolates grouped together in a clade with PPSMV2 and FMV (Figure 7). The aa sequence of the NP shared the highest identity with PPSMV2 with $71.7 \%$. According to the species demarcation of ICTV, a difference of $25 \%$ in the aa sequence of the NP indicates a new species (Elbeaino et al., 2018). Therefore, this virus represents a new emaravirus species and was tentatively called pea associated emaravirus (PaEV).

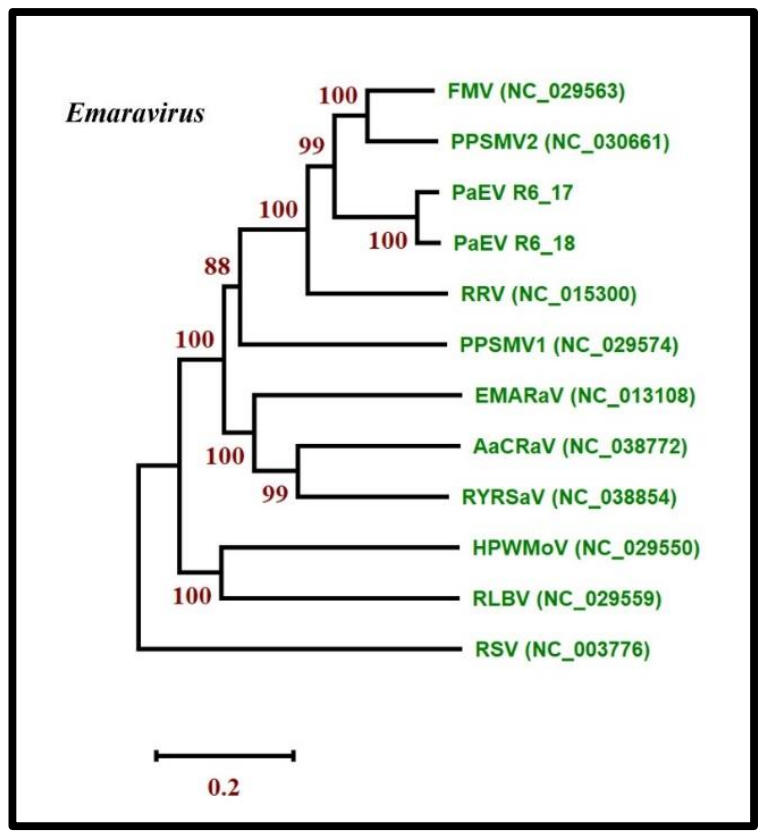

Figure 7: Neighbour joining trees (NJ) of the nucleocapsid protein (NP) of the two isolates of pea associated emaravirus (PaEV) and representative emaraviruses detected in German pea. The phylogenetic trees are based alignments of the amino acid sequences. The sequences were 
aligned with Clustal $\mathrm{W}$ and NJ trees constructed with MEGA X. The percentage of the bootstrap values above $50 \%$ (1,000 replications) are shown at the nodes. The names of the viruses are as follow: AcCRaV: actinidia chlorotic ringspot-associated emaravirus, EMARaV: European mountain ash ringspot-associated emaravirus, FMV: fig mosaic emaravirus, HPWMEV: high plains wheat mosaic emaravirus, PaEV: pea associated emaravirus, PPSMV1: pigeonpea sterility mosaic emaravirus 1, PPSMV2: pigeonpea sterility mosaic emaravirus 2, RLBV: raspberry leaf blotch emaravirus, RRV: rose rosette emaravirus, RSV: rice stripe virus (Tenuivirus, Phenuiviridae) and RYRSaV: redbud yellow ringspot-associated emaravirus. The Genbank accession numbers are mentioned in the brackets.

\subsubsection{Narnaviridae: Pea associated mitoviruses}

In Landkreis Rostock, four mitoviruses (family: Narnaviridae) were detected in the SP and aSP in 2017. An isolate of Sclerotinia sclerotiorum mitovirus 4 (SsMV4) was also identified in the same region. The isolate shared $96.3 \%$ aa identity to SsMV4 from New Zealand (AGC24233). In addition, three mitoviruses provisionally called pea associated mitovirus 1, 2 and 3 (PaMV1, PaMV2 and PaMV3) were identified. The PaMV1-CDS shared 68.8\% nt identity to its closest match Erysiphe necator mitovirus 3 (EnMV3; KY420040), that was identified from the grape powdery mildew fungus Erysiphe necator (Schwein.) (syn. Uncinula necator (Schw.)) that was described in the USA recently (Pandey et al., 2018). Based on the RdRp region, PaMV1 shared 65.1\% aa identity with EnMV3. PaMV2CDS had 36\% nt identity to Rhizoctonia solani mitovirus 6 (RsMV6; KP900915 from the USA). Based on the aa sequences, PaMV1 and RsMV6 share only $40.2 \%$ aa identity. PaMV3-CDS was closely related to Entomophthora muscae mitovirus 5 (EnmuMV5; MK682524) with $40 \%$ nt identity and $24.3 \%$ aa identity in the $\mathrm{RdRp}$ region.

\subsubsection{Secoviridae}

In Münster 2017, a new putative member of the family Secoviridae was identified. The virus was closely related to strawberry mottle virus (SMoV) and lettuce secovirus 1 (LSV1), two unassigned putative Secoviridae viruses. The virus was tentatively called surrounding non-Legume secovirus (snLSV). Based on the protease-polymerase region (Pro-Pol), this virus shared the closest aa identity to LSV1 with $67.3 \%$ identity while the CP region showed only $31.6 \%$ aa identity to LSV1. In addition, a new strain of carrot torradovirus 1 (CaTV1) was discovered that shared $95.9 \%$ aa identity based on the Pro-Pol region and $95.4 \%$ identity based on the $\mathrm{CP}$ region with the CaTV1 strain celery that was recently identified from Germany (MK063924 and MK063925).

A new strain of red clover nepovirus A (RCNVA) (genus: Nepovirus; subfamily: Comovirinae; family: Secoviridae) was detected in Landkreis Rostock 2017. This new strain had $96.5 \%$ identity based on the aa sequence of the Pro-Pol region with RCNVA-B46 from the Czech Republic (MG253828) and a CP aa identity of 83.2\% (MG253829).

A divergent cherry leaf roll virus (CLRV) (genus: Nepovirus) was identified in Salzlandkreis-1 in 2018. The virus shared closest identity with CLRV isolates from New Zealand, with RNA1 sharing $82.4 \%$ nt identity to CLRV isolate KC937022 and RNA2 sharing 
$80 \%$ nt identity to KC937029. The aa sequence of the Pro-Pol region shared $97 \%$ identity to KC937022 while the CP region had only $89.8 \%$ aa identity with KC937029.

\subsubsection{Betaflexiviridae}

A divergent strain of red clover carlavirus 1 (RCCV1) (genus: Carlavirus; subfamily: Quinvirinae; family: Betaflexiviridae), was identified only once in 2018 in one location (Kreis Stormarn). The partial RdRp sequence shared $85.3 \%$ aa identity with RCCV1 (MG596238 and MG596239) from the Czech Republic. Interestingly, the complete coding sequence of an isolate of Heracleum latent virus (HLV) (genus: Vitivirus; subfamily: Trivirinae, family: Betaflexiviridae), was identified in snL of Münster 2017. Based on the CP sequence, this isolate shared $90.9 \%$ identity to HLV from Scotland (NC_039087) on the nt level and $96.4 \%$ identity based on the aa sequence. The RdRp region shared only $58.4 \%$ nt identity to grapevine virus B (GVB; MF991949) and 58.9\% aa identity, respectively. A partial sequence of a chordovirus (subfamily: Trivirinae) was also detected in the SnL of Münster 2017, tentatively called snL chordovirus (snLCV). The partial sequence shared 73.2\% nt identity with carrot chordovirus 1 (CaChV1; NC_025469). Additionally, a partial waikavirus sequence was detected in the snL of Kreis Stormarn in 2018 with that showed 73.9\% nt identity to bellflower vein chlorosis virus (BVCV; NC_027915) from South Korea.

\subsubsection{Bromoviridae}

Cucumber mosaic virus (CMV) (genus: Cucumovirus; family: Bromoviridae) was found in Salzlandkreis-2's sL in 2018. The three viral RNAs shared 99.1 to $99.5 \%$ nt identity to various CMV isolates (HE793685 from France, AF416900 from USA, EF202597 from China). Additionally, two new ilarviruses were identified in the $\mathrm{snL}$ samples of Trenthorst in 2016 and the $s L$ of Landkreis Rostock in 2018. The putative CP aa sequence of the $\mathrm{snL}$ ilarvirus shared closest identity to asparagus virus 2 (AV2; NC_011807, from Mexico) with 81.1\% aa identity. The $\mathrm{SL}$ ilarvirus CP was closely related to ageratum latent virus from Australia (AgLV; NC_022129) with 60.3\% aa identity.

\subsubsection{Other viruses}

Black grass cryptic virus 2 (BGCV2) was detected in Salzlandkreis-1 in 2016; RNA1 shared 99\% nt identity with an isolate probably from the UK (NC_026799) while its RdRp shared $100 \%$ aa identity. A turnip vein-clearing virus (TVCV), member of subgroup 3 of the genus Tobamovirus (family: Virgaviridae), was identified in Salzlandkreis-2's snL in 2018. The assembled complete CDS of Salzlandkreis-2 TVCV isolate shared 95.5\% nt identity with TVCV strain NZ-587 from New Zealand (accession no. JN205074). The amino acid sequences shared between 97.7 and $99.4 \%$ with their homologs of strain NZ-587. White clover cryptic virus 2 (WCCV-2; genus Betapartitivirus) was identified in sL of Kreis Stormarn 2017 and Münster 2018, with 98.6\% and 98.5\% nt identity to WCCV-2 from Australia (MH427306). Six white clover mosaic virus (WCIMV) isolates, genus: Potexvirus; family: Alphaflexiviridae, were identified in the sL namely in white clover (Trifolium 
repens) and shared 99.2 to 99.3 to the WCMV-PV1 isolate from the Czech Republic (MG600296). Finally, a sequence with $81.3 \% \mathrm{nt}$ identity to Wuhan insect virus 21 (WHIV21) (KX883227) from China was detected in sL of Salzlandkreis-1 2018.

\subsubsection{Spatial and temporal differences in viral populations}

The spatial and temporal compositions of the viromes in the different regions over a period of three growing seasons show, perhaps not unsurprisingly, similarities as well as fundamental differences. For example, PEMV1, PEMV2 and PNYDV were the viruses that could be located in all regions (but not in every season; Figure 8 ). The polerovirus TuYV was detected in all regions except for the Landkreis Rostock region (Figure 8). This location is close to the Baltic Sea with winds blown inland from the seaside; therefore, aphids as virus vectors are unlikely to carry viruses into the crops.

Interestingly, some viruses could only be located in very few regions. For example, WCMV was detected in four regions i.e., Salzlandkreis-2, Münster, Kreis Stormarn and Landkreis Rostock whereas several other viruses were only present in two different regions. BYMV was detected in two regions i.e., Salzlandkreis-1 and Salzlandkreis-2. BGCV2 was in Salzlandkreis-1 and Landkreis Rostock. CIYVV was in Salzlandkreis-2 and Münster. PSbMV was detected Salzlandkreis-2 and Kreis Stormarn. RCEV 1 and RCUV were detected in two regions i.e., Münster and Landkreis Meißen. SbDV and WCCV2 were detected Münster and Kreis Stormarn. Furthermore, BLRV and PaEV were detected only in one region (Salzlandkreis-2 and Landkreis Meißen, respectively). 


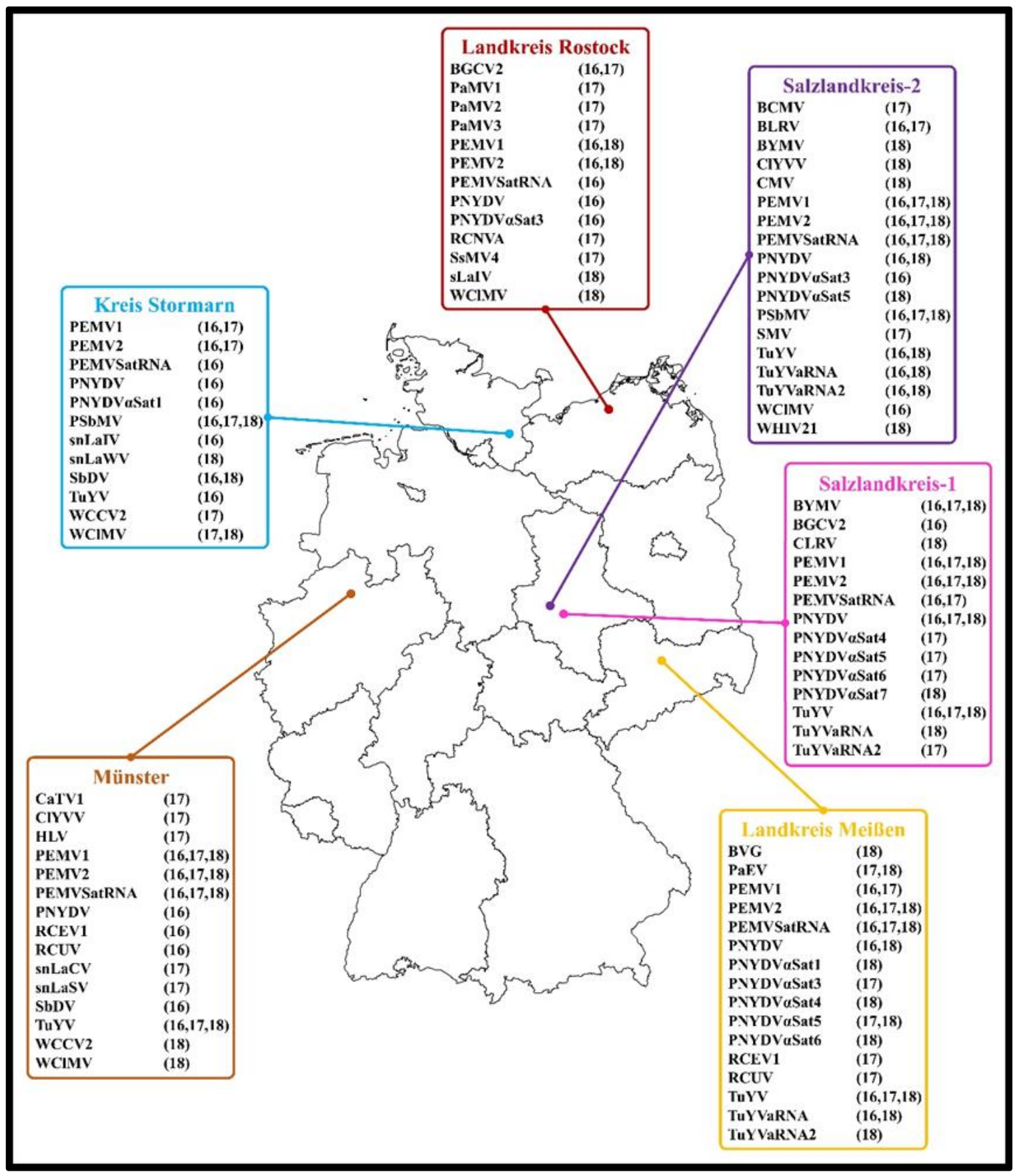

Figure 8: List of viruses and their associated nucleic acids detected in each of the six German pea growing regions sampled over three seasons 2016, 2017 and 2018. The seasons in which the viruses were detected are mentioned in brackets. The names of the viruses and their associated nucleic acids are: BVG: barley virus G, BCMV: bean common mosaic virus, BLRV: bean leafroll virus, BYMV: bean yellow mosaic virus, BGCV2: black grass cryptic virus 2, CaTV1: carrot torradovirus 1, CLRV: cherry leaf roll virus, CIYVV: clover yellow vein virus, CMV: cucumber mosaic virus, HLV: Heracleum latent virus, PaMV1: pea associated mitovirus 1, PaMV2: pea associated mitovirus 2, PaMV3: pea associated mitovirus 3, PaEV: pea associatted emaravirus, PEMV1: pea enation mosaic virus 1, PEMV2: pea enation mosaic virus 2, PEMVsatRNA: pea enation mosaic virus satellite RNA, PNYDV $\alpha$ Sat1: pea necrotic yellow dwarf alphasatellite 1, PNYDVaSat3: pea necrotic yellow dwarf alphasatellite 3, PNYDVaSat4: pea necrotic yellow dwarf alphasatellite 4, PNYDVaSat5: pea necrotic yellow dwarf alphasatellite 5, PNYDVaSat6: pea necrotic yellow dwarf 
alphasatellite 6, PNYDVaSat7: pea necrotic yellow dwarf alphasatellite 7, PNYDV: pea necrotic yellow dwarf virus, PSbMV: pea seed-borne mosaic virus, RCCV1: red clover carlavirus 1, RCEV1: red clover enamovirus 1, RCNVA: red clover nepovirus a, RCUV: red clover umbravirus, SsMV4: Sclerotinia sclerotiorum mitovirus 4, SLaIV: SL associated ilarvirus, SnLaCV: SnL associated chordovirus, snLalV: snL associated ilarvirus, snLaSV: snL associated secoviridae, snLaWV: snL associated waikavirus, SbDV: soybean dwarf virus, SMV: soybean mosaic virus, TVCV: turnip veinclearing virus, TuYV: turnip yellows virus, TuYVaRNA: turnip yellows virus associated RNA, TuYVaRNA2: turnip yellows virus associated RNA 2, WCCV2: white clover cryptic virus 2, WCIMV: white clover mosaic virus, WHIV21: Wuhan insect virus 21.

Other viruses were found in only one season in one region i.e., BVG, BCMV, CaTV1, CLRV, CMV, HLV, RCCV1, RCNVA, SMV, TVCV, WHIV21 and all mitoviruses. So far, many of these viruses have not yet been included in standard monitoring programs of pea diseases, therefore the incidence and impact of these viruses on pea and other legume crops is currently unknown.

PEMV1, PEMV2 and their satellites were not detected in 2017 in Landkreis Rostock and in 2018 in Kreis Stormarn. In 2018, PEMV1 was not detected in Landkreis Rostock, however PEMV2 and the satellite RNA were present. TuYV was detected in all three seasons in Salzlandkreis-1, Münster and Landkreis Meißen, however after its detection in Kreis Stormarn in 2016, it could not be detected in 2017 and 2018. Meanwhile, TuYV could not be identified in Salzlandkreis-2 in 2017.

PNYDV, an emerging nanovirus in Germany, was detected in all the regions in 2016 (Figure 8). PNYDV was detected during in all three seasons in Salzlandkreis-1 and Landkreis Meißen, consistent with previous monitoring. PNYDV was not detected in the other four regions in 2017; however, in 2018, it was again detected in Salzlandkreis-2. In 2016 and 2017, WCIMV could be found in Salzlandkreis-2 and Kreis Stormarn while in 2018 it was detected again in Kreis Stormarn with additional confirmations in Münster and Landkreis Rostock. PSbMV was detected in all seasons in Salzlandkreis-2 and Kreis Stormarn. Although BLRV in one region, it was detected for two successive season 2016 and 2017 but could not be found in 2018. PaEV was also detected for two seasons in 2017 and 2018.

Taken together, when we look at the spatial distribution of viruses, we can only observe minor differences in the viral community compositions. No major differences could be observed between the regions as e.g., a total of 36 virus incidences were detected in Salzlandkreis-2 followed by Münster with 33 virus incidences (over the three seasons). A total of 28 virus incidences were reported from Salzlandkreis-1, 25 for both Kreis Stormarn and Landkreis Meißen and the lowest rate for Landkreis Rostock (20).

The temporal virus incidences appear to be relatively stable over the successive seasons 2016, 2017 and 2018 with 53, 56 and 54 virus incidences, respectively (Supplementary Table S4). 


\subsection{Discussion}

This is the first HTS-based study designed to describe the pea virome in Germany. In addition to the focus on viruses infecting pea crops, we also explored spatio-temporal aspects across six different production regions in Germany over three years. We also examined as potential virus reservoirs non-pea plants surrounding the pea crops, including those related to peas (belonging to the Fabaceae) and plants that were completely unrelated. This distinguishes our study from many metagenomics studies that focused on either just one crop plant, one production area, one season or combination thereof. We believe that this study demonstrates the importance of spatio-temporal aspects into metagenomics studies in order to draw a more complete picture of all the viruses present.

To discover viruses with different genomes and prevent bias we used a ribosomal RNA depleted total RNA (Pecman et al., 2017). As demonstrated, this method detected RNA viruses with both a plus and negative sense genome, and viruses with a DNA genome, namely PNYDV and its associated satellites. We discovered a surprisingly high number of viruses in the different pools with some present in high abundance, i.e., CMV, PEMV1, PEMV2, PEMVSatRNA, TUYV, TUYVaRNA1 and TuYVaRNA2. The recovered reads were pool-dependent as well as dependent on the viral genome, virus titre and incidence within the pool (Supplementary Table S3, Figure 3). All these viruses are positive single-stranded viruses. Interestingly, dsRNA viruses, i.e., partitivirids, and the new negative sense RNA emaravirus were also detected using the ribo-depletion method, despite a low number of reads.

\subsubsection{Detection of known viruses in the pea pools}

As expected, we were able to detect and confirm by RT-PCR the presence of many known pea viruses that have been described for Germany previously, i.e., PEMV1, PEMV2, TuYV, PNYDV, BLRV, SbDV, PSbMV and CMV. This is in line with previous surveys and observations (data not shown). PEMV1 and PEMV2 are usually the most commonly found viruses in German pea crops (Ziebell, 2017). PEMV is associated with enation and mosaic symptoms on infected plants and can lead to severe yield losses (Clement, 2006). Furthermore, mixed infections of PEMV1 and PEMV2 are well documented in pea samples (Hagedorn \& Khan, 1984; Brault et al., 2010).

The second most prevalent virus detected was TuYV. It was found in peas and also in the snL pools. TuYV is known to infect peas but is of major concern for rapeseed in Germany (Graichen \& Schliephake, 1999; Gaafar \& Ziebell, 2019b). Although, we have no direct evidence that surrounding non-legumes are reservoirs for TuYV isolates that infect peas, in greenhouse experiments we demonstrated that TuYV isolates originating from peas can infect rapeseed and vice versa (data not shown). It is therefore very likely that rapeseed crops, other members of the Brassicaceae family as well as a large number 
of common weeds and wild species host range are alternative hosts for pea-infecting poleroviruses such as TuYV (Stevens et al., 1994).

BLRV is another luteovirus that has been known for a long time to infect peas in Germany (Quantz \& Volk, 1954). It has been reported from many other countries e.g., Australia, Greece, India and USA and can infect various other legumes causing symptoms of stunting and leaf rolling (Reddy et al., 1979; Vemulapati et al., 2010; van Leur \& Kumari, 2011; Chatzivassiliou et al., 2016). Control of BLRV is best managed using host plant resistance (van Leur et al., 2013), however, BLRV-resistant green and protein pea varieties may not be widely available at the present. As with other phloem-limited luteoviruses, BLRV is transmitted by aphids in a non-persistent manner with pea aphids (Acyrthosiphon pisum) being one of the most efficient vectors (Clement, 2006). Another luteovirus, SbDV, is also known to infect peas and other legumes in Germany (Grigoras et al., 2010a; Abraham et al., 2007). The virus was reported from many countries causing economic losses on soybean (Glycine max L.) (Phibbs et al., 2004; Tamada et al., 1969). The virus can cause several symptoms e.g., yellowing, dwarfing, downward curling, rugosity and reduction in growth (Tamada et al., 1969; Abraham et al., 2007) but it is unclear whether peas with BLRV resistance can be infected with other closely-related poleroviruses.

Ten years ago, PNYDV was first discovered in Germany (Grigoras et al., 2010a). In the following years, PNYDV was only detected in two German regions in Saxony and Saxony-Anhalt, as well as in neighbouring Austria. However, 2016 saw the first countrywide outbreak of this virus in Germany and PNYDV was detected also in other European countries (e.g. Denmark and The Netherlands) (Gaafar et al., 2016; Gaafar et al., 2017; Ziebell, 2017; Gaafar et al., 2018a). Effects on infected plants are severe and can cause high yield losses (Saucke et al., 2019). PNYDV is an increasing threat to legume production in Europe as no PNYDV-resistant plant varieties have been identified yet (data not shown). Additionally, we believe the high mutation rate, reassortment and recombination rates of nanoviruses like PNYDV may lead to the appearance of novel strains (Grigoras et al., 2010b; Grigoras et al., 2014). An increasing number of nanovirus-associated singlestranded circular DNA alphasatellites have been reported in recent years in legumes such as Sophora alopecuroides L., Vicia cracca L. and Apiaceae members such as Petroselinum crispum (Mill.) Fuss, although their biological relevance is still unclear (Gallet et al., 2018; Vetten et al., 2019; Heydarnejad et al., 2017).

The seed- and aphid-transmissible PSBMV has also been reported from many countries, including Germany (Khetarpal \& Maury, 1987; Latham \& Jones, 2001). However, in Germany, PSbMV is not seen as a major constraint of pea production as the provision of "clean" seed material and close surveillance of pea seed production sites has helped to reduce PSbMV incidence below those described for other countries. It is interesting that one of the two sites in which we detected PSBMV is a trial site for heritage material. The second region in which we detected PSbMV is in closer proximity to a protein pea breeding site in which we detected PSbMV in previous surveys (data not 
shown) therefore viruliferous aphids may be vectors in this region. The symptoms of PSbMV are reported to be mild and transitory in pea resulting in limited detection of the virus in the field (Khetarpal \& Maury, 1987). Other symptoms can include slight chlorosis, stunting, shortening, veins swelling and leaflets' downward rolling (Khetarpal \& Maury, 1987). Seed symptoms of coat staining, coat splitting, and reduced seed size caused by PSbMV were observed in New Zealand, USA and Australia in the 1980s. As a result, a program to breed resistance was initiated leading to the release of pea varieties highly tolerant to PSbMV (Fletcher et al., 1989; Russell, 1994). Similar breeding programs were also successfully developed in Australia (van Leur et al., 2013).

The presence of CMV in the $S L$ was not surprising, as CMV is a wide-spread virus, with an extremely wide host range; CMV can be transmitted by many aphid species, and cause severe losses in legumes (Palukaitis et al., 1992; Fletcher, 1993; Garcla-Arenal \& Palukaitis, 2009; Tao et al., 2002). CMV was reported before in German peas and other legumes but does not seem to be of major concern (Schmidt, 1981).

Although as being reported as present in Germany e.g., widespread on faba beans or clover plants, we could not find any detailed information on BYMV or CIYVV naturally infecting peas in Germany. BYMV has a wide host range compared to other potyviruses including legumes and ornamentals (Guyatt et al., 1996; Nakazono-Nagaoka et al., 2009). Additionally, it can be transmitted by more than 20 aphid species causing symptoms including mosaic, necrosis and yellowing resulting in severe yield losses (Guyatt et al., 1996; Nakazono-Nagaoka et al., 2009). The pathogenicity and serotypes of the BYMV differ from one strain to another (Bos, 1970; Barnett, 1987). Clover yellow vein virus (CIYVV) has a host range overlapping with BYMV and often confused with it as they are closed serologically (Barnett, 1987; Nakazono-Nagaoka et al., 2009).

\subsubsection{New players in German peas}

Our study highlights that there are many pea viruses present in Germany that have neither been described previously and are not being monitored: RCEV1, RCUV, SSMV4, and two associated nucleic acids i.e., PEMVSatRNA and TuYVaRNA.

Interestingly, RCEV1 and RCUV were not only detected in peas but also in the sL (but not in the same location and not in the same season) which might indicate that surrounding, perennial legumes can be a virus reservoir for peas. The possibility of a mixed infection with both viruses was confirmed for red clover (Trifolium pratense L.) in the Czech Republic (Koloniuk et al. personal comm.). Such mixed infections between luteovirids and umbraviruses is common i.e., PEMV1 and PEMV2.

The mitovirus SsMV4 was found to infect Sclerotinia sclerotiorum (Lib.) de Bary, a widespread plant pathogenic fungus causing white mould disease especially in peas, lentils and beans (McKenzie \& Morrall, 1975; Purdy, 1979; Bardin \& Huang, 2001; Bolton et al., 2006; Nibert et al., 2018). . A study showed that SsMV4 in combination with two 
other mitoviruses i.e., Sclerotinia sclerotiorum mitovirus 2 (SsMV2) and Sclerotinia sclerotiorum mitovirus 3 (SSMV3), reduced the in-vitro growth and virulence of $S$. sclerotiorum on cabbage, common bean, oilseed rape and tomato (Khalifa \& Pearson, 2013).

For the first time in Germany we also discovered numerous virus associated nucleic acid sequences. PEMVSatRNA, a small linear single stranded RNA satellite, has also been extracted from peas in the USA (Demler \& Zoeten, 1989). PEMVSatRNA appears not influence aphid transmission, particle morphology, or symptom expression in peas but can reduce the severity of symptoms on the indicator plant Nicotiana benthamiana (Demler \& Zoeten, 1989; Demler et al., 1994). Whether the PEMVSatRNA detected in Germany can modulate symptom expression of PEMV in its natural host $P$. sativum remains to be investigated. We also discovered PNYDV-associated alphasatellites 1 and 3 . PNYDVaSat1 was previously detected in Austria, while PNYDVaSat3 was detected in both Austria and Denmark (Gaafar et al., 2018a) In addition, four new alphasatellites were detected (discussed later). Finally, TuYVaRNA was also detected in association with TuYV. We discovered TuYVaRNA recently associated with TuYV from rapeseed in Germany (Gaafar \& Ziebell, 2019b) but its role in symptom modulation, host range determination or vector transmission also remains to be investigated.

\subsubsection{New viruses and associated satellites - what are the risks?}

A new emaravirus i.e., PaEV, three new mitoviruses associated with peas i.e., PaMV1, PaMV2 and PaMV3, and novel associated nucleic acids in peas PNYDVaSat4, PNYDVaSat5, PNYDVaSat6 and TuYVaRNA2 were detected in this survey. PaEV was detected in German peas for two successive seasons in Landkreis Meißen, therefore we a quite confident that this virus is established in this region and might pose a risk. However, due to our pooling strategy, we are unable to attribute symptoms associated with PaEMV or to recover infectious virus material from the samples. Generally, emaraviruses can infect trees and deciduous shrubs. In the USA and Canada, the emaravirus rose rosette virus (RRV), mite-transmitted by Phyllocoptes fructiphilus Kiefer (Acari: Eriophyidae), causes extreme damage to roses leading to plant death within a short period of time (Olson et al., 2017). RRV and its vector were placed on the $A 1$ alert list by the European and Mediterranean Plant Protection Organisation (EPPO, 2019). Two other emaraviruses have been reported from legumes. On pigeonpea (Cajanus cajan L.; Fabaceae) sterility mosaic disease (SMD) is caused by pigeonpea sterility mosaic emaravirus 1 and pigeonpea sterility mosaic emaravirus 2 (Elbeaino et al., 2014, 2015). These emaraviruses can also be transmitted by eriophyid mites (Aceria cajani Chann.) (Elbeaino et al., 2015; Patil \& Kumar, 2015). The total number of segments of our PaEV isolate are unknown as the virus full genome could not be recovered due to its low number of reads in the pools. In future monitoring programs, the distribution of $\mathrm{PaEV}$ will be evaluated to assess the risk that this virus might pose. 
Three new mitoviruses were found in the pea pools i.e., PaMV1, PaMV2 and PaMV3. Mitoviruses are wide spread in plants and their infection to pathogenic fungi is often associated with virulence reduction (Bruenn et al., 2015; Wu et al., 2007; Xie \& Ghabrial, 2012). The fungi which these new mitoviruses can infect are currently unknown and need more investigation. Also, it is important to understand the role of these mitoviruses as they may be used as biocontrol for fungal infections that in future could help to reduce the impact of fungal diseases in peas (powdery mildew, downy mildew, Aphanomyces, fusarium root rot).

In addition to the previously described PNYDV alphasatellites 1 and 3, we discovered four new PNYDV-associated alphasatellites. Alphasatellites rely on their helper virus for spread as they do not encode a coat protein (Briddon et al., 2018). The presence of alphasatellites is associated with reduced infectivity of faba bean necrotic yellows virus (a nanovirus) or in the case of begomoviruses alphasatellites reduced or intensified symptoms and/or reduced virus genomic titre or betasatellite (Timchenko et al., 2006; Paprotka et al., 2010; Mar et al., 2017; Idris et al., 2011; Kon et al., 2009). The Rep proteins encoded by begomoviruses' alphasatellites were found to suppress transcriptional gene silencing or post-transcriptional gene silencing (Nawaz-UI-Rehman et al., 2010; Abbas et al., 2019). The role of these alphasatellites in unknown and their association with nanoviruses and other viruses e.g., babuviruses and begomoviruses require clarification.

Additional to the reported TuYVaRNA1, we detected a new TuYV associated RNA, TuYVaRNA2. The effects of these associated RNAs on TuYV transmission and infection need more investigation as previous studies showed that beet western yellows associated RNA (strain ST9) increases the severity of on beet western yellows virus and is not associated with the virions' escaping the phloem (Sanger et al., 1994).

\subsubsection{Non-crop plants: Reservoirs for viruses infected crops}

In our study, we analysed plants (leguminous and non-leguminous plants) surrounding the pea fields to investigate potential virus reservoirs. We were able to detect various viruses in those plant pools that may pose a threat to peas as well as production of other crops.

\subsubsection{In the surrounding legumes:}

Not surprisingly, in surrounding legumes we were able to detect viruses which are known to infect other legumes i.e., BCMV, SMV, WCCV2 and WCIMV in addition to CMV and SbDV (discussed above). BCMV is well known to infect Phaseolus beans causing common mosaic or black root disease depending on the host, virus strain and the environmental conditions (Drijfhout \& Bos, 1977). BCMV is a seed-borne virus, aphid transmissible and can be transmitted mechanically. A previous study showed that BCMV NL1 strain could not infect peas (Drijfhout \& Bos, 1977). 
SMV is also distributed worldwide and is a main threat for soybean production in many countries e.g., Japan and USA (Hill \& Whitham, 2014). Its symptoms including mosaic, leaf distortion, leaf deformation and seed damage. SMV reduces germination, seed size, oil content and nodulation, and total yield in soybean (Hill \& Whitham, 2014; Ross, 1977). SMV can be seed transmitted or by aphid vectors (Li et al., 2018). Besides soybean, it can infect other legumes including pea and was found in German faba beans before (Nandakishor et al., 2017). WCCV2, a symptomless cryptic virus, has been previously detected in German in white clover (Trifolium repens) (Lesker et al., 2013) and in many other countries like Japan, Korea, New Zealand and USA (Zhao et al., 2016; Ido et al., 2012; Park et al., 2017; Nakabayashi et al., 2002; Bos et al., 1959). The virus can infect several legumes including pea, white clover, red clover (Trifolium pratense) and nonlegumes e.g., garlic mustard (Alliaria petiolata) causing mosaic symptoms. Interestingly, this virus was also detected by HTS in Australian honey bee populations (Roberts et al., 2018). WCIMV is usually mechanically transmitted and may be seed transmitted with few records of insect transmission (Garrett, 1991).

In our survey, we identified new viruses in the surrounding legumes; i.e., RCCV1, RCNVA, WCIMoV and WHIV21 in addition to RCEV1 and RCUV (discussed above). RCCV1 is avirus which was present in the $S L$ pool and which may also infect peas. In a previous 2016 survey using antibodies developed for detection of red clover vein mosaic virus (RCVMV)-like carlaviruses, the presence of a carlavirus was suggested in several pea samples but the exact virus species was not determined (Ziebell, 2017). Koloniuk and colleagues recently identified the genome of RCCV1 and found that it shares the capsid protein sequence with RCVMV (Koloniuk et al., personal comm.). RCNVA is a new virus that was identified in red clover (Trifolium pratense L.) plant in the Czech Republic (Koloniuk et al., 2018). It was detected only once in 2018 in the sL pool of Landkreis Rostock. The host range of RCNVA is currently unknown.

Based on the sequences available on NCBI, WCIMoV was only detected in Korea in white clover, however no more information is available. The finding of WHIV21 in a plant is interesting as another strain (WHCCI13077) was only reported in China from insect tissues (Shi et al., 2016a). The virus is taxonomically unassigned to any virus family and its role is currently unknown.

A new ilarvirus, which we named sLaIV was detected in the sL. Although the exact host of sLaIV is currently unknown due to our pooling strategy, this virus will be included in future surveys to investigate incidence and potential host plants. To our best knowledge, sLaIV is only the second ilarvirus to naturally infect legumes after tobacco streak virus (Kaiser, 1982).

\subsubsection{In the surrounding non-legumes:}

In the pools of surrounding non-legume plants, we were able to detect various viruses that were described previously for Germany i.e., CaTV1, CLRV and TuYV. CaTV1 was detected recently in celery plants exhibiting chlorotic ringspots, mosaic and strong 
yellowing symptoms (Gaafar \& Ziebell, 2019a) Similarly, the divergent strain of CLRV detected in this study is not new to Germany, as it has been previously detected in carrots (data not published). The presence of other strains of CLRV have been previously reported from German forest trees (beech and birch) (Jones et al., 1990; Rebenstorf, 2005). This virus is widely distributed and has a wide natural host range including woody and herbaceous plants (Büttner et al., 2011). CLRV may be transmitted by seed, pollen, as well mechanically including grafting. CLRV can cause a wide range of symptoms depending on the host and strain, for example in cherry trees it can cause a delay in leaf formation, upward leaf rolling, it can also delay flowering, reduce fruit production and can eventually kill the tree (Cropley, 1961) Finding CLRV in the surrounding non-legumes is not surprising as it was also detected previously in rhubarb (Rheum rhaponticum; family: Polygonaceae) in Britain (Tomlinson \& Walkey, 1967). CLRV was also transmitted mechanically to peas and French beans in addition to many other economically important plants (Tomlinson \& Walkey, 1967).

BVG, BGCV2, HLV and TVCV are new reports for Germany. BVG was detected recently in the Netherlands, a neighbouring country of Germany, and infects switchgrass (Panicum virgatum L.) (Kumar et al., 2018a). The presence of this virus in Germany may be important for the production of barley and other cereal crops (Jo et al., 2018a). Another cryptic virus i.e., BGCV2 was detected in the snL. BGCV2 is a recently identified virus sequence from black grass (Alopecurus myosuroides Huds.) based on NCBI data (no publication available).

Viruses belonging to the Betaflexiviridae family were also detected in the "non-legume weed pools". We were able to determine the complete coding sequence of HLV for the first time. This virus was only detected once before in Scotland and appears to be widely spread in wild hogweed (Heracleum sphondylium L.; Apiaceae), with no obvious leaf symptoms (Bem \& Murant, 1979). However, it was possible to infect many other plants experimentally including carrot (Daucus carota L.), maize (Zea mays L.), and cowpea (Vigna unguiculata (L.) Walp.), another legume. HLV can be transmitted mechanically or by aphids (Bem \& Murant, 1979) On experimental plants symptoms such as chlorosis, mottling, necrotic rings or spots or vein clearing could be observed (Bem \& Murant, 1979). Although the virus did not show symptoms on most of the infected plants, the effect of the virus on plant production is currently unknown.

TVCV was detected one time in the snL of Salzlandkreis-2. Previously, TVCV was detected in several countries e.g., France, New Zealand and USA (Cardin et al., 2009; Cohen et al., 2012; Lockhart et al., 2008). It infects different species including turnip (Brassica rapa L.), penstemon (Penstemon digitalis Nutt. ex Sims.) Plantago major L., and kidney weed (Dichondra repens L.) and the crop plant Actinidia chinensis Planch. (kiwifruit) causing different symptoms including vein clearing, red foliar ringspots, leaf deformation and plant stunting (Dorokhov et al., 1994; Cohen et al., 2012; Lartey et al., 1994; Cardin et al., 2009; Lockhart et al., 2008; Blouin et al., 2013). 
We also detected several new viruses in the snL i.e., snLaCV, snLaIV, snLaSV and snLaWV. As the hosts of these viruses are not yet defined, the importance of these newly identified virus sequences i.e., sLaIV, snLaCV, snLaIV, snLaSV and snLaWV is unknown.

Our study identified several new viruses, virus strains and isolates that had not been reported before. Our findings help to improve pea virus surveys as the range of target viruses needs to be extended and the sequence data that we generated helps to improve our knowledge about virus variation thus improving taxonomy and finetuning of species demarcation criteria. The detection of another divergent CaTV1 (a torradovirus) as well as the divergent nepoviruses CLRV and RCNVA, indicate that the ICTV species demarcation criteria set for the family Secoviridae may require alteration. The criteria might also consider other genomic regions e.g., the full aa sequence of the poly proteins and the nt sequences of the untranslated regions as suggested by (Verbeek et al., 2010; Gaafar \& Ziebell, 2019a).

Our sequence data may also help to improve public databases as we were able to supply several full-length genomes. Since our BLAST results and phylogenetic trees indicate closest sequence matches from isolates that were described only from other continents more HTS studies are needed in Europe to improve local sequence databases and subsequent sequence analyses.

Whilst we acknowledge the strength of HTS in identifying known and unknown viruses of crops, our pooling strategy also has disadvantages. Firstly, we cannot obtain detailed information on the viruses infecting a single plant without back testing each specimen in the pool. Secondly, in some case it was not possible to recover the full-length viral sequence using this method. Thirdly, this approach does not allow us to link symptom data on individual plants with the viruses found in the pooled samples without back testing each specimen in the pool although subsequent testing of retained plant tissue will be insightful.

We believe that our survey data is of great interest for plant breeders (breeding for virus resistance), diagnosticians and pest risk analysist but it also demonstrates clearly the challenges of metagenomic HTS studies in the framework of virus diagnostics (laboratory and bioinformatics challenges, result interpretation, biological significance, pest risk analyses and data sharing) (Olmos et al., 2018).

\subsection{Conclusions}

In conclusion, our method of using rRNA-depleted total RNA extracts from pooled plant tissue in combination with HTS, bioinformatic analysis and molecular confirmation has increased the speed and breadth of virus detection in one crop species in Germany over three seasons. This method enabled the detection of a range of viruses regardless of their genome type. After sequencing pea samples, we identified sequences representing thirty-five viruses, many of which were represented by nearly full genomes. As expected, well recognised pea viruses were detected in this study, including members of the 
Luteoviridae, Nanoviridae, Potyviridae and Tombusviridae families. In addition, 25 new viruses associated with pea, non-crop legumes and non-legume plants were revealed, some unexpected and as yet unexplained. Much work is still needed to reveal the importance and context of these new host /virus associations.

We found PEMV1 and PEMV2 were the dominant virus species in pea which is consistent with what has been observed in the past. We also found some viruses had little similarity with known viruses and suggest they could be categorized as new viruses. For example, a new emaravirus was consistently detected in peas over two of the survey seasons. Other viruses were also detected in pea or in Germany first time and their importance to pea has yet to be determined. We believe the data from this study provides a comprehensive and improved overview of viruses present in German pea fields. For the newly detected viruses, further work is needed to determine the complete host range of these viruses, their effect on hosts and their likely vectors. It is also necessary to further investigate different locations and environments to increase our understanding of the virodiversity of these new viruses not only of pea but other legumes globally. The information produced on the long list of important viral pathogens and new virus species and strains present in the German pea fields should contribute to alert local governments and to establish sanitation measures to prevent viral transmission. Moreover, this study provides more evidence on the benefits of HTS and metagenomics in an important crop such as pea and the potential to develop similar virus databases in different crop fields. 


\subsection{Supplementary}

Table S1: The metadata records (the plant, symptoms, and the average temperature) of the six German pea growing regions sampled over three seasons 2016, 2017 and 2018.

\begin{tabular}{|c|c|c|c|c|c|}
\hline Sample & Season & Category & Plant & Symptoms & Temperature \\
\hline R1-16-01 & 2016 & SP & Pisum sativum & severe dwarfing, yellowing & \multirow{30}{*}{$19^{\circ} \mathrm{C}$} \\
\hline $\mathrm{R} 1-16-02$ & 2016 & SP & Pisum sativum & yellowing, top stunting, crippled pods & \\
\hline R1-16-03 & 2016 & SP & Pisum sativum & yellowing, top stunting, crippled pods & \\
\hline R1-16-04 & 2016 & SP & Pisum sativum & severe dwarfing, chlorotic spots & \\
\hline R1-16-05 & 2016 & SP & Pisum sativum & top yellowing, chlorotic spots & \\
\hline R1-16-06 & 2016 & SP & Pisum sativum & $\begin{array}{l}\text { top yellowing, chlorotic spots, top } \\
\text { stunting }\end{array}$ & \\
\hline R1-16-07 & 2016 & SP & Pisum sativum & $\begin{array}{c}\text { top yellowing, vein clearing, top } \\
\text { dwarfing }\end{array}$ & \\
\hline R1-16-08 & 2016 & SP & Pisum sativum & yellowing, top stunting & \\
\hline R1-16-09 & 2016 & SP & Pisum sativum & $\begin{array}{l}\text { enation symptoms on top, top } \\
\text { yellowing, crippled pods }\end{array}$ & \\
\hline R1-16-10 & 2016 & SP & Pisum sativum & $\begin{array}{c}\text { severe dwarfing, yellowing, vein } \\
\text { clearing }\end{array}$ & \\
\hline R1-16-11 & 2016 & aSP & Pisum sativum & no obvious symptoms & \\
\hline R1-16-12 & 2016 & aSP & Pisum sativum & no obvious symptoms & \\
\hline R1-16-13 & 2016 & aSP & Pisum sativum & no obvious symptoms & \\
\hline R1-16-14 & 2016 & aSP & Pisum sativum & no obvious symptoms & \\
\hline R1-16-15 & 2016 & aSP & Pisum sativum & no obvious symptoms & \\
\hline R1-16-16 & 2016 & aSP & Pisum sativum & no obvious symptoms & \\
\hline R1-16-17 & 2016 & aSP & Pisum sativum & no obvious symptoms & \\
\hline R1-16-18 & 2016 & aSP & Pisum sativum & no obvious symptoms & \\
\hline R1-16-19 & 2016 & aSP & Pisum sativum & no obvious symptoms & \\
\hline R1-16-20 & 2016 & aSP & Pisum sativum & no obvious symptoms & \\
\hline R1-16-21 & 2016 & sL & $N A^{*}$ & NA & \\
\hline R1-16-22 & 2016 & sL & NA & NA & \\
\hline R1-16-23 & 2016 & $s L$ & NA & NA & \\
\hline R1-16-24 & 2016 & SL & NA & NA & \\
\hline R1-16-25 & 2016 & sL & NA & NA & \\
\hline R1-16-26 & 2016 & snL & Euphorbia sp. & no obvious symptoms & \\
\hline R1-16-27 & 2016 & snL & Poacae sp. & no obvious symptoms & \\
\hline R1-16-28 & 2016 & snL & Viola sp. & no obvious symptoms & \\
\hline R1-16-29 & 2016 & $\mathrm{snL}$ & Unknown & mottling, chlorotic spots & \\
\hline R1-16-30 & 2016 & snL & Chenopodium sp. & chlorotic spots, chlorotic rings & \\
\hline R1-17-01 & 2017 & SP & Pisum sativum & dwarfing, pod deformation & \multirow{5}{*}{$18^{\circ} \mathrm{C}$} \\
\hline R1-17-02 & 2017 & SP & Pisum sativum & $\begin{array}{l}\text { top dwarfing, mottling, pod } \\
\text { deformation }\end{array}$ & \\
\hline R1-17-03 & 2017 & SP & Pisum sativum & mottling & \\
\hline R1-17-04 & 2017 & SP & Pisum sativum & top yellowing and dwarfing & \\
\hline R1-17-05 & 2017 & SP & Pisum sativum & top yellowing and dwarfing, mottling & \\
\hline
\end{tabular}




\begin{tabular}{|c|c|c|c|c|c|}
\hline R1-17-06 & 2017 & SP & Pisum sativum & top yellowing and dwarfing, mottling & \\
\hline R1-17-07 & 2017 & SP & Pisum sativum & top dwarfing, mottling & \\
\hline R1-17-08 & 2017 & SP & Pisum sativum & $\begin{array}{c}\text { top dwarfing, mottling, pod } \\
\text { deformation }\end{array}$ & \\
\hline R1-17-09 & 2017 & SP & Pisum sativum & top yellowing and dwarfing & \\
\hline R1-17-10 & 2017 & SP & Pisum sativum & top dwarfing, yellowing & \\
\hline R1-17-11 & 2017 & aSP & Pisum sativum & no obvious symptoms & \\
\hline R1-17-12 & 2017 & aSP & Pisum sativum & no obvious symptoms & \\
\hline R1-17-13 & 2017 & aSP & Pisum sativum & no obvious symptoms & \\
\hline R1-17-14 & 2017 & aSP & Pisum sativum & no obvious symptoms & \\
\hline R1-17-15 & 2017 & aSP & Pisum sativum & no obvious symptoms & \\
\hline R1-17-16 & 2017 & aSP & Pisum sativum & no obvious symptoms & \\
\hline R1-17-17 & 2017 & aSP & Pisum sativum & no obvious symptoms & \\
\hline R1-17-18 & 2017 & aSP & Pisum sativum & no obvious symptoms & \\
\hline R1-17-19 & 2017 & aSP & Pisum sativum & no obvious symptoms & \\
\hline R1-17-20 & 2017 & aSP & Pisum sativum & no obvious symptoms & \\
\hline R1-17-21 & 2017 & $s L$ & Trifolium sp. & no obvious symptoms & \\
\hline R1-17-22 & 2017 & $s L$ & Trifolium sp. & no obvious symptoms & \\
\hline R1-17-23 & 2017 & $s L$ & Trifolium sp. & no obvious symptoms & \\
\hline R1-17-24 & 2017 & $s L$ & Trifolium sp. & no obvious symptoms & \\
\hline R1-17-25 & 2017 & $s L$ & Melilotus sp. & no obvious symptoms & \\
\hline R1-17-26 & 2017 & $\operatorname{snL}$ & Euphorbia sp. & no obvious symptoms & \\
\hline R1-17-27 & 2017 & $\operatorname{snL}$ & Chenopodium sp. & no obvious symptoms & \\
\hline R1-17-28 & 2017 & snL & Brassica napus & chlorotic spots, reddening & \\
\hline R1-17-29 & 2017 & snL & Poacae sp. & no obvious symptoms & \\
\hline R1-17-30 & 2017 & snL & Brassica napus & chlorotic spots & \\
\hline R1-18-01 & 2018 & SP & Pisum sativum & top dwarfing & \multirow{19}{*}{$22^{\circ} \mathrm{C}$} \\
\hline R1-18-02 & 2018 & SP & Pisum sativum & dwarfing & \\
\hline R1-18-03 & 2018 & SP & Pisum sativum & top dwarfing & \\
\hline R1-18-04 & 2018 & SP & Pisum sativum & top yellowing, pod deformation & \\
\hline R1-18-05 & 2018 & SP & Pisum sativum & short internodes & \\
\hline R1-18-06 & 2018 & SP & Pisum sativum & dwarfing & \\
\hline R1-18-07 & 2018 & SP & Pisum sativum & top dwarfing & \\
\hline R1-18-08 & 2018 & SP & Pisum sativum & enation, top yellowing & \\
\hline R1-18-09 & 2018 & SP & Pisum sativum & leaf rolling, stunting & \\
\hline R1-18-10 & 2018 & SP & Pisum sativum & short internodes & \\
\hline R1-18-11 & 2018 & aSP & Pisum sativum & no obvious symptoms & \\
\hline R1-18-12 & 2018 & aSP & Pisum sativum & no obvious symptoms & \\
\hline R1-18-13 & 2018 & aSP & Pisum sativum & no obvious symptoms & \\
\hline R1-18-14 & 2018 & aSP & Pisum sativum & no obvious symptoms & \\
\hline R1-18-15 & 2018 & aSP & Pisum sativum & no obvious symptoms & \\
\hline R1-18-16 & 2018 & aSP & Pisum sativum & no obvious symptoms & \\
\hline R1-18-17 & 2018 & aSP & Pisum sativum & no obvious symptoms & \\
\hline R1-18-18 & 2018 & aSP & Pisum sativum & no obvious symptoms & \\
\hline R1-18-19 & 2018 & aSP & Pisum sativum & no obvious symptoms & \\
\hline
\end{tabular}




\begin{tabular}{|c|c|c|c|c|c|}
\hline R1-18-20 & 2018 & aSP & Pisum sativum & no obvious symptoms & \\
\hline R1-18-21 & 2018 & $s L$ & Chenopodium sp. & no obvious symptoms & \\
\hline R1-18-22 & 2018 & sL & Robinia sp. & no obvious symptoms & \\
\hline R1-18-23 & 2018 & sL & Robinia sp. & no obvious symptoms & \\
\hline R1-18-24 & 2018 & $s L$ & Lathyrus sp. & no obvious symptoms & \\
\hline R1-18-25 & 2018 & SL & Robinia sp. & no obvious symptoms & \\
\hline R1-18-26 & 2018 & $\operatorname{snL}$ & Brassica napus & no obvious symptoms & \\
\hline R1-18-27 & 2018 & snL & Sambucus nigra & mosaic & \\
\hline R1-18-28 & 2018 & snL & Convolvulaceae sp. & red ring spots & \\
\hline R1-18-29 & 2018 & snL & Chenopodium sp. & leaf reddening, mottling & \\
\hline R1-18-30 & 2018 & snL & Unknown & no obvious symptoms & \\
\hline \multicolumn{6}{|c|}{ Salzlandkreis-2 (pea heritage collection site) } \\
\hline Sample & Season & Category & Plant & Symptoms & Temperature \\
\hline R2-16-01 & 2016 & SP & Pisum sativum & yellowing, enation, dwarfing & \multirow{29}{*}{$19^{\circ} \mathrm{C}$} \\
\hline R2-16-02 & 2016 & SP & Pisum sativum & top yellowing, severe leaf rolling & \\
\hline R2-16-03 & 2016 & SP & Pisum sativum & yellowing, severe dwarfing & \\
\hline R2-16-04 & 2016 & SP & Pisum sativum & vein clearing, enation, leaf deformation & \\
\hline R2-16-05 & 2016 & SP & Pisum sativum & enation & \\
\hline R2-16-06 & 2016 & SP & Pisum sativum & top yellowing, leaf rolling, vein clearing & \\
\hline R2-16-07 & 2016 & SP & Pisum sativum & yellowing, severe dwarfing, necrosis & \\
\hline R2-16-08 & 2016 & SP & Pisum sativum & top yellowing, top dwarfing & \\
\hline R2-16-09 & 2016 & SP & Pisum sativum & $\begin{array}{l}\text { leaf deformation, red edges, chlorotic } \\
\text { spots }\end{array}$ & \\
\hline R2-16-10 & 2016 & SP & Pisum sativum & enation & \\
\hline R2-16-11 & 2016 & aSP & NA & NA & \\
\hline R2-16-12 & 2016 & aSP & NA & NA & \\
\hline R2-16-13 & 2016 & aSP & NA & NA & \\
\hline$R 2-16-14$ & 2016 & aSP & NA & NA & \\
\hline R2-16-15 & 2016 & aSP & NA & NA & \\
\hline R2-16-16 & 2016 & aSP & NA & NA & \\
\hline R2-16-17 & 2016 & aSP & NA & NA & \\
\hline R2-16-18 & 2016 & aSP & NA & NA & \\
\hline R2-16-19 & 2016 & aSP & NA & NA & \\
\hline R2-16-20 & 2016 & aSP & NA & NA & \\
\hline R2-16-21 & 2016 & $s L$ & Medicago doliata & no obvious symptoms & \\
\hline$R 2-16-22$ & 2016 & $s L$ & $\begin{array}{c}\text { Trifolium } \\
\text { tomentosum }\end{array}$ & no obvious symptoms & \\
\hline$R 2-16-23$ & 2016 & $s L$ & $\begin{array}{c}\text { Robinia } \\
\text { pseudoacacia }\end{array}$ & mottling, mosaic & \\
\hline R2-16-24 & 2016 & $s L$ & Trifolium sp. & no obvious symptoms & \\
\hline$R 2-16-25$ & 2016 & $s L$ & $\begin{array}{c}\text { Trifolium } \\
\text { squarrosum }\end{array}$ & leaf reddening & \\
\hline R2-16-26 & 2016 & snL & Amaranthus sp. & no obvious symptoms & \\
\hline R2-16-27 & 2016 & snL & Galium aparine & red midrib & \\
\hline R2-16-28 & 2016 & snL & Fumaria vaillantii & yellowing, leaf reddening & \\
\hline R2-16-29 & 2016 & snL & Chenopodium sp. & chlorotic spots & \\
\hline
\end{tabular}




\begin{tabular}{|c|c|c|c|c|c|}
\hline R2-16-30 & 2016 & $\mathrm{snL}$ & $\begin{array}{l}\text { Taraxacum } \\
\text { officinale }\end{array}$ & no obvious symptoms & \\
\hline R2-17-01 & 2017 & SP & Pisum sativum & $\begin{array}{l}\text { top dwarfing, chlorotic spots, pod } \\
\text { deformation }\end{array}$ & \multirow{30}{*}{$19^{\circ} \mathrm{C}$} \\
\hline $\mathrm{R} 2-17-02$ & 2017 & SP & Pisum sativum & leaf deformation & \\
\hline $\mathrm{R} 2-17-03$ & 2017 & SP & Pisum sativum & yellowing, leaf and pod deformantion & \\
\hline R2-17-04 & 2017 & SP & Pisum sativum & dwarfing & \\
\hline R2-17-05 & 2017 & SP & Pisum sativum & mosaic, top dwarfing & \\
\hline R2-17-06 & 2017 & SP & Pisum sativum & chlorotic spots & \\
\hline R2-17-07 & 2017 & SP & Pisum sativum & yellowing & \\
\hline R2-17-08 & 2017 & SP & Pisum sativum & yellowing, pod deformation & \\
\hline R2-17-09 & 2017 & SP & Pisum sativum & mosaic, pod deformation & \\
\hline $\mathrm{R} 2-17-10$ & 2017 & SP & Pisum sativum & severe yellowing, pod deformation & \\
\hline R2-17-11 & 2017 & aSP & Pisum sativum & no obvious symptoms & \\
\hline $\mathrm{R} 2-17-12$ & 2017 & aSP & Pisum sativum & no obvious symptoms & \\
\hline R2-17-13 & 2017 & aSP & Pisum sativum & no obvious symptoms & \\
\hline R2-17-14 & 2017 & aSP & Pisum sativum & no obvious symptoms & \\
\hline R2-17-15 & 2017 & aSP & Pisum sativum & no obvious symptoms & \\
\hline $\mathrm{R} 2-17-16$ & 2017 & aSP & Pisum sativum & no obvious symptoms & \\
\hline R2-17-17 & 2017 & aSP & Pisum sativum & no obvious symptoms & \\
\hline R2-17-18 & 2017 & aSP & Pisum sativum & no obvious symptoms & \\
\hline R2-17-19 & 2017 & aSP & Pisum sativum & no obvious symptoms & \\
\hline$R 2-17-20$ & 2017 & aSP & Pisum sativum & no obvious symptoms & \\
\hline R2-17-21 & 2017 & $s L$ & Vicia sp. & yellowing, mottling & \\
\hline$R 2-17-22$ & 2017 & $s L$ & Vicia sp. & yellowing, mottling & \\
\hline R2-17-23 & 2017 & $s L$ & Trifolium sp. & no obvious symptoms & \\
\hline R2-17-24 & 2017 & $s L$ & Trifolium sp. & no obvious symptoms & \\
\hline$R 2-17-25$ & 2017 & $s L$ & Vicia $s p$ & no obvious symptoms & \\
\hline$R 2-17-26$ & 2017 & snL & Chenopodium sp. & yellowing, mottling & \\
\hline R2-17-27 & 2017 & snL & Polygonaceae sp. & chlorotic spots & \\
\hline R2-17-28 & 2017 & snL & Apiaceae sp. & no obvious symptoms & \\
\hline R2-17-29 & 2017 & $\operatorname{snL}$ & Apiaceae sp. & no obvious symptoms & \\
\hline R2-17-30 & 2017 & snL & Anethum sp. & no obvious symptoms & \\
\hline R3-18-01 & 2018 & SP & Pisum sativum & enation & \multirow{12}{*}{$22^{\circ} \mathrm{C}$} \\
\hline R3-18-02 & 2018 & SP & Pisum sativum & enation & \\
\hline R3-18-03 & 2018 & SP & Pisum sativum & enation & \\
\hline R3-18-04 & 2018 & SP & Pisum sativum & enation & \\
\hline R3-18-05 & 2018 & SP & Pisum sativum & chlorosis, yellowing & \\
\hline R3-18-06 & 2018 & SP & Pisum sativum & enation & \\
\hline R3-18-07 & 2018 & $\mathrm{SP}$ & Pisum sativum & enation & \\
\hline R3-18-08 & 2018 & SP & Pisum sativum & enation & \\
\hline R3-18-09 & 2018 & SP & Pisum sativum & enation & \\
\hline R3-18-10 & 2018 & SP & Pisum sativum & enation & \\
\hline R3-18-11 & 2018 & aSP & Pisum sativum & no obvious symptoms & \\
\hline R3-18-12 & 2018 & aSP & Pisum sativum & no obvious symptoms & \\
\hline
\end{tabular}




\begin{tabular}{|c|c|c|c|c|c|}
\hline R3-18-13 & 2018 & aSP & Pisum sativum & no obvious symptoms & \\
\hline R3-18-14 & 2018 & aSP & Pisum sativum & no obvious symptoms & \\
\hline R3-18-15 & 2018 & aSP & Pisum sativum & no obvious symptoms & \\
\hline R3-18-16 & 2018 & aSP & Pisum sativum & no obvious symptoms & \\
\hline R3-18-17 & 2018 & aSP & Pisum sativum & no obvious symptoms & \\
\hline R3-18-18 & 2018 & aSP & Pisum sativum & no obvious symptoms & \\
\hline R3-18-19 & 2018 & aSP & Pisum sativum & no obvious symptoms & \\
\hline R3-18-20 & 2018 & aSP & Pisum sativum & no obvious symptoms & \\
\hline R3-18-21 & 2018 & sL & Medicago doliata & no obvious symptoms & \\
\hline R3-18-22 & 2018 & $s L$ & $\begin{array}{l}\text { Trifolium } \\
\text { incarnatum }\end{array}$ & no obvious symptoms & \\
\hline R3-18-23 & 2018 & sL & Trifolium pratense & no obvious symptoms & \\
\hline R3-18-24 & 2018 & $s L$ & Trigonella caerulea & no obvious symptoms & \\
\hline R3-18-25 & 2018 & sL & $\begin{array}{l}\text { Medicago } \\
\text { muricoleptis }\end{array}$ & no obvious symptoms & \\
\hline R3-18-26 & 2018 & $\mathrm{snL}$ & Phacelia sp. & no obvious symptoms & \\
\hline R3-18-27 & 2018 & snL & $\begin{array}{l}\text { Taraxacum } \\
\text { officinalis }\end{array}$ & leaf reddening & \\
\hline R3-18-28 & 2018 & snL & Plantago major & no obvious symptoms & \\
\hline R3-18-29 & 2018 & snL & Brassica oleracea & yellowing & \\
\hline R3-18-30 & 2018 & snL & Brassica oleracea & mottling, leaf reddening & \\
\hline \multicolumn{6}{|c|}{ Münster (pea breeding site) } \\
\hline Sample & Season & Category & Plant & Symptoms & Temperature \\
\hline R3-16-01 & 2016 & SP & Pisum sativum & top yellowing & \multirow{21}{*}{$17^{\circ} \mathrm{C}$} \\
\hline R3-16-02 & 2016 & SP & Pisum sativum & $\begin{array}{c}\text { severe chlorosis, top stunting, leaf } \\
\text { deformation }\end{array}$ & \\
\hline R3-16-03 & 2016 & SP & Pisum sativum & chlorotic spots, top dwarfing, enation & \\
\hline R3-16-04 & 2016 & SP & Pisum sativum & curly pods, necrosis, top yellowing & \\
\hline R3-16-05 & 2016 & SP & Pisum sativum & enation, yellowing & \\
\hline R3-16-06 & 2016 & SP & Pisum sativum & $\begin{array}{c}\text { vein clearing, deformation, necrosis, } \\
\text { enation }\end{array}$ & \\
\hline R3-16-07 & 2016 & SP & Pisum sativum & $\begin{array}{c}\text { vein clearing, chlorotic spots, necrosis, } \\
\text { enation }\end{array}$ & \\
\hline R3-16-08 & 2016 & SP & Pisum sativum & enation, mosaic & \\
\hline R3-16-09 & 2016 & SP & Pisum sativum & $\begin{array}{l}\text { severe dwarfing, yellowing, pod } \\
\text { deformation, enation }\end{array}$ & \\
\hline R3-16-10 & 2016 & SP & Pisum sativum & severe stunting, yellowing & \\
\hline R3-16-11 & 2016 & aSP & Pisum sativum & no obvious symptoms & \\
\hline R3-16-12 & 2016 & aSP & Pisum sativum & no obvious symptoms & \\
\hline R3-16-13 & 2016 & aSP & Pisum sativum & no obvious symptoms & \\
\hline R3-16-14 & 2016 & aSP & Pisum sativum & no obvious symptoms & \\
\hline R3-16-15 & 2016 & aSP & Pisum sativum & no obvious symptoms & \\
\hline R3-16-16 & 2016 & aSP & Pisum sativum & no obvious symptoms & \\
\hline R3-16-17 & 2016 & aSP & Pisum sativum & no obvious symptoms & \\
\hline R3-16-18 & 2016 & aSP & Pisum sativum & no obvious symptoms & \\
\hline R3-16-19 & 2016 & aSP & Pisum sativum & no obvious symptoms & \\
\hline R3-16-20 & 2016 & aSP & Pisum sativum & no obvious symptoms & \\
\hline R3-16-21 & 2016 & $s L$ & Vicia sp. & no obvious symptoms & \\
\hline
\end{tabular}




\begin{tabular}{|c|c|c|c|c|c|}
\hline R3-16-22 & 2016 & $s L$ & Vicia sp. & no obvious symptoms & \\
\hline R3-16-23 & 2016 & $s L$ & Trifolium pratense & no obvious symptoms & \\
\hline R3-16-24 & 2016 & $s L$ & Trifolium repens & no obvious symptoms & \\
\hline R3-16-25 & 2016 & sL & Vicia sp. & reddish pods & \\
\hline R3-16-26 & 2016 & snL & Chenopodium sp. & chlorotic spots & \\
\hline R3-16-27 & 2016 & $\operatorname{snL}$ & Galinsoga parviflora & no obvious symptoms & \\
\hline R3-16-28 & 2016 & $\operatorname{snL}$ & Rumex sp. & no obvious symptoms & \\
\hline R3-16-29 & 2016 & $\operatorname{snL}$ & $\begin{array}{c}\text { Capsella bursa- } \\
\text { pastoris }\end{array}$ & no obvious symptoms & \\
\hline R3-16-30 & 2016 & snL & $\begin{array}{l}\text { Matricaria } \\
\text { chamomilla }\end{array}$ & no obvious symptoms & \\
\hline R3-17-01 & 2017 & SP & Pisum sativum & yellowing, mottling & \multirow{30}{*}{$19^{\circ} \mathrm{C}$} \\
\hline R3-17-02 & 2017 & SP & Pisum sativum & enation, mottling, pod deformation & \\
\hline R3-17-03 & 2017 & SP & Pisum sativum & enation, yellowing & \\
\hline R3-17-04 & 2017 & SP & Pisum sativum & top dwarfing, pod deformation & \\
\hline R3-17-05 & 2017 & SP & Pisum sativum & enation & \\
\hline R3-17-06 & 2017 & SP & Pisum sativum & severe mottling & \\
\hline R3-17-07 & 2017 & SP & Pisum sativum & enation & \\
\hline R3-17-08 & 2017 & SP & Pisum sativum & top dwarfing and mottling & \\
\hline R3-17-09 & 2017 & SP & Pisum sativum & top dwarfing, pod deformation & \\
\hline R3-17-10 & 2017 & SP & Pisum sativum & yellowing, enation & \\
\hline R3-17-11 & 2017 & aSP & Pisum sativum & no obvious symptoms & \\
\hline R3-17-12 & 2017 & aSP & Pisum sativum & no obvious symptoms & \\
\hline R3-17-13 & 2017 & aSP & Pisum sativum & no obvious symptoms & \\
\hline R3-17-14 & 2017 & aSP & Pisum sativum & no obvious symptoms & \\
\hline R3-17-15 & 2017 & aSP & Pisum sativum & no obvious symptoms & \\
\hline R3-17-16 & 2017 & aSP & Pisum sativum & no obvious symptoms & \\
\hline R3-17-17 & 2017 & aSP & Pisum sativum & no obvious symptoms & \\
\hline R3-17-18 & 2017 & aSP & Pisum sativum & no obvious symptoms & \\
\hline R3-17-19 & 2017 & aSP & Pisum sativum & no obvious symptoms & \\
\hline R3-17-20 & 2017 & aSP & Pisum sativum & no obvious symptoms & \\
\hline R3-17-21 & 2017 & $s L$ & Trifolium sp. & no obvious symptoms & \\
\hline R3-17-22 & 2017 & $s L$ & Melilotus sp. & no obvious symptoms & \\
\hline R3-17-23 & 2017 & sL & Trifolium sp. & no obvious symptoms & \\
\hline R3-17-24 & 2017 & $s L$ & Trifolium sp. & no obvious symptoms & \\
\hline R3-17-25 & 2017 & $s L$ & Vicia $s p$ & no obvious symptoms & \\
\hline R3-17-26 & 2017 & snL & Unknown & yellowing, mottling & \\
\hline R3-17-27 & 2017 & snL & Galium sp. & chlorotic spots & \\
\hline R3-17-28 & 2017 & snL & Geranium sp. & yellowing & \\
\hline R3-17-29 & 2017 & snL & Aegopodium sp. & no obvious symptoms & \\
\hline R3-17-30 & 2017 & $\mathrm{snL}$ & Chenopodium sp. & chlorotic spots & \\
\hline R3-18-01 & 2018 & SP & Pisum sativum & enation, mosaic, leaf deformation & \multirow{4}{*}{$22^{\circ} \mathrm{C}$} \\
\hline R3-18-02 & 2018 & SP & Pisum sativum & enation, mosaic & \\
\hline R3-18-03 & 2018 & SP & Pisum sativum & enation, mosaic & \\
\hline R3-18-04 & 2018 & SP & Pisum sativum & yellowing, mottling, pod deformation & \\
\hline
\end{tabular}




\begin{tabular}{|c|c|c|c|c|c|}
\hline R3-18-05 & 2018 & SP & Pisum sativum & enation, mosaic, leaf deformation & \\
\hline R3-18-06 & 2018 & SP & Pisum sativum & enation, mosaic, leaf rolling & \\
\hline R3-18-07 & 2018 & SP & Pisum sativum & enation, mosaic & \\
\hline R3-18-08 & 2018 & SP & Pisum sativum & yellowing, mottling & \\
\hline R3-18-09 & 2018 & SP & Pisum sativum & chlorotic spots, mosaic & \\
\hline R3-18-10 & 2018 & SP & Pisum sativum & chlorotic spots, mosaic & \\
\hline R3-18-11 & 2018 & aSP & Pisum sativum & no obvious symptoms & \\
\hline R3-18-12 & 2018 & aSP & Pisum sativum & no obvious symptoms & \\
\hline R3-18-13 & 2018 & aSP & Pisum sativum & no obvious symptoms & \\
\hline R3-18-14 & 2018 & aSP & Pisum sativum & no obvious symptoms & \\
\hline R3-18-15 & 2018 & aSP & Pisum sativum & no obvious symptoms & \\
\hline R3-18-16 & 2018 & aSP & Pisum sativum & no obvious symptoms & \\
\hline R3-18-17 & 2018 & aSP & Pisum sativum & no obvious symptoms & \\
\hline R3-18-18 & 2018 & aSP & Pisum sativum & no obvious symptoms & \\
\hline R3-18-19 & 2018 & aSP & Pisum sativum & no obvious symptoms & \\
\hline R3-18-20 & 2018 & aSP & Pisum sativum & no obvious symptoms & \\
\hline R3-18-21 & 2018 & sL & Oxalis stricta & no obvious symptoms & \\
\hline R3-18-22 & 2018 & sL & Trifolium repens & no obvious symptoms & \\
\hline R3-18-23 & 2018 & $s L$ & Trifolium repens & mottling & \\
\hline R3-18-24 & 2018 & sL & Vicia $s p$ & no obvious symptoms & \\
\hline R3-18-25 & 2018 & sL & Vicia sp. & chlorotic lesions & \\
\hline R3-18-26 & 2018 & snL & Solanum nigrum & no obvious symptoms & \\
\hline R3-18-27 & 2018 & $\mathrm{snL}$ & $\begin{array}{l}\text { Hypericum } \\
\text { officinalis }\end{array}$ & no obvious symptoms & \\
\hline R3-18-28 & 2018 & $\mathrm{snL}$ & Unknown & no obvious symptoms & \\
\hline R3-18-29 & 2018 & snL & Chenopodium sp. & chlorotic spots & \\
\hline R3-18-30 & 2018 & $\mathrm{snL}$ & Euphorbia sp. & no obvious symptoms & \\
\hline \multicolumn{6}{|c|}{ Kreis Stormarn (organic farming site) } \\
\hline Sample & Season & Category & Plant & Symptoms & Temperature \\
\hline R4-16-01 & 2016 & SP & Pisum sativum & top yellowing, leaf deformation & \multirow{15}{*}{$17^{\circ} \mathrm{C}$} \\
\hline R4-16-02 & 2016 & SP & Pisum sativum & top mottling, leaf deformation & \\
\hline R4-16-03 & 2016 & SP & Pisum sativum & vein clearing & \\
\hline R4-16-04 & 2016 & SP & Pisum sativum & top yellowing, dwarfing, mottling & \\
\hline R4-16-05 & 2016 & SP & Pisum sativum & $\begin{array}{l}\text { stunting, top yellowing, leaf } \\
\text { deformation }\end{array}$ & \\
\hline R4-16-06 & 2016 & SP & Pisum sativum & yellowing, leaf rolling & \\
\hline R4-16-07 & 2016 & SP & Pisum sativum & stunting, top yellowing, leaf rolling & \\
\hline R4-16-08 & 2016 & SP & Pisum sativum & top yellowing, dwarfing & \\
\hline R4-16-09 & 2016 & SP & Pisum sativum & leaf mottling, yellowing, brittle leaves & \\
\hline R4-16-10 & 2016 & SP & Pisum sativum & enation & \\
\hline R4-16-11 & 2016 & aSP & Pisum sativum & no obvious symptoms & \\
\hline R4-16-12 & 2016 & aSP & Pisum sativum & no obvious symptoms & \\
\hline R4-16-13 & 2016 & aSP & Pisum sativum & no obvious symptoms & \\
\hline R4-16-14 & 2016 & aSP & Pisum sativum & no obvious symptoms & \\
\hline R4-16-15 & 2016 & aSP & Pisum sativum & no obvious symptoms & \\
\hline
\end{tabular}




\begin{tabular}{|c|c|c|c|c|c|}
\hline R4-16-16 & 2016 & aSP & Pisum sativum & no obvious symptoms & \\
\hline R4-16-17 & 2016 & aSP & Pisum sativum & no obvious symptoms & \\
\hline R4-16-18 & 2016 & aSP & Pisum sativum & no obvious symptoms & \\
\hline R4-16-19 & 2016 & aSP & Pisum sativum & no obvious symptoms & \\
\hline R4-16-20 & 2016 & aSP & Pisum sativum & no obvious symptoms & \\
\hline R4-16-21 & 2016 & $s L$ & Trifolium repens & no obvious symptoms & \\
\hline R4-16-22 & 2016 & $s L$ & Trifolium repens & no obvious symptoms & \\
\hline$R 4-16-23$ & 2016 & $s L$ & Trifolium repens & no obvious symptoms & \\
\hline R4-16-24 & 2016 & $s L$ & Trifolium repens & no obvious symptoms & \\
\hline R4-16-25 & 2016 & $s L$ & Trifolium repens & no obvious symptoms & \\
\hline R4-16-26 & 2016 & snL & Chenopodium sp. & chlorotic spots & \\
\hline R4-16-27 & 2016 & snL & Apiaceae sp. & no obvious symptoms & \\
\hline R4-16-28 & 2016 & snL & Apiaceae sp. & no obvious symptoms & \\
\hline R4-16-29 & 2016 & snL & Lamiaceae sp. & no obvious symptoms & \\
\hline R4-16-30 & 2016 & snL & Urtica sp. & no obvious symptoms & \\
\hline R4-17-01 & 2017 & SP & Pisum sativum & top dwarfing, pod deformation & \multirow{29}{*}{$17^{\circ} \mathrm{C}$} \\
\hline R4-17-02 & 2017 & SP & Pisum sativum & mottling, pod deformation & \\
\hline R4-17-03 & 2017 & SP & Pisum sativum & yellowing & \\
\hline R4-17-04 & 2017 & SP & Pisum sativum & top dwarfing, pod deformation & \\
\hline R4-17-05 & 2017 & SP & Pisum sativum & yellowing & \\
\hline R4-17-06 & 2017 & SP & Pisum sativum & yellowing, pod deformation & \\
\hline R4-17-07 & 2017 & SP & Pisum sativum & mottling, pod deformation & \\
\hline R4-17-08 & 2017 & SP & Pisum sativum & $\begin{array}{l}\text { yellowing, leaf hardening and rolling, } \\
\text { pod deformation }\end{array}$ & \\
\hline R4-17-09 & 2017 & SP & Pisum sativum & yellowing, chlorotic spots & \\
\hline $\mathrm{R} 4-17-10$ & 2017 & SP & Pisum sativum & top dwarfing, pod deformation & \\
\hline R4-17-11 & 2017 & aSP & Pisum sativum & no obvious symptoms & \\
\hline R4-17-12 & 2017 & aSP & Pisum sativum & no obvious symptoms & \\
\hline R4-17-13 & 2017 & aSP & Pisum sativum & no obvious symptoms & \\
\hline R4-17-14 & 2017 & aSP & Pisum sativum & no obvious symptoms & \\
\hline R4-17-15 & 2017 & aSP & Pisum sativum & no obvious symptoms & \\
\hline R4-17-16 & 2017 & aSP & Pisum sativum & no obvious symptoms & \\
\hline R4-17-17 & 2017 & aSP & Pisum sativum & no obvious symptoms & \\
\hline R4-17-18 & 2017 & aSP & Pisum sativum & no obvious symptoms & \\
\hline R4-17-19 & 2017 & aSP & Pisum sativum & no obvious symptoms & \\
\hline R4-17-20 & 2017 & aSP & Pisum sativum & no obvious symptoms & \\
\hline R4-17-21 & 2017 & $s L$ & Trifolium sp. & no obvious symptoms & \\
\hline R4-17-22 & 2017 & $s L$ & Trifolium sp. & leaf reddening & \\
\hline R4-17-23 & 2017 & $s L$ & Trifolium sp. & yellowing, leaf reddening & \\
\hline R4-17-24 & 2017 & $s L$ & Trifolium sp. & no obvious symptoms & \\
\hline R4-17-25 & 2017 & $s L$ & Trifolium sp. & yellowing, leaf reddening & \\
\hline R4-17-26 & 2017 & $\mathrm{snL}$ & Rumex sp. & red ring spots & \\
\hline R4-17-27 & 2017 & $\mathrm{snL}$ & Unknown & yellowing & \\
\hline R4-17-28 & 2017 & $\operatorname{snL}$ & Unknown & no obvious symptoms & \\
\hline R4-17-29 & 2017 & snL & Aegopodium sp. & red ring spots & \\
\hline
\end{tabular}




\begin{tabular}{|c|c|c|c|c|c|}
\hline R4-17-30 & 2017 & snL & Aegopodium sp. & severe yellowing & \\
\hline R4-18-01 & 2018 & SP & Pisum sativum & yellowing & \multirow{30}{*}{$20^{\circ} \mathrm{C}$} \\
\hline R4-18-02 & 2018 & SP & Pisum sativum & yellowing, leaf and pod deformation & \\
\hline R4-18-03 & 2018 & SP & Pisum sativum & yellowing, leaf and pod deformation & \\
\hline R4-18-04 & 2018 & SP & Pisum sativum & yellowing, mottling & \\
\hline R4-18-05 & 2018 & SP & Pisum sativum & yellowing, mottling, leaf rolling & \\
\hline R4-18-06 & 2018 & SP & Pisum sativum & severe yellowing, pod deformation & \\
\hline R4-18-07 & 2018 & SP & Pisum sativum & yellowing, mottling, top dwarfing & \\
\hline R4-18-08 & 2018 & SP & Pisum sativum & yellowing, mottling & \\
\hline R4-18-09 & 2018 & SP & Pisum sativum & chlorotic spots & \\
\hline $\mathrm{R} 4-18-10$ & 2018 & SP & Pisum sativum & yellowing, mottling, leaf rolling & \\
\hline R4-18-11 & 2018 & aSP & Pisum sativum & no obvious symptoms & \\
\hline R4-18-12 & 2018 & aSP & Pisum sativum & no obvious symptoms & \\
\hline R4-18-13 & 2018 & aSP & Pisum sativum & no obvious symptoms & \\
\hline R4-18-14 & 2018 & aSP & Pisum sativum & no obvious symptoms & \\
\hline R4-18-15 & 2018 & aSP & Pisum sativum & no obvious symptoms & \\
\hline R4-18-16 & 2018 & aSP & Pisum sativum & no obvious symptoms & \\
\hline R4-18-17 & 2018 & aSP & Pisum sativum & no obvious symptoms & \\
\hline R4-18-18 & 2018 & aSP & Pisum sativum & no obvious symptoms & \\
\hline R4-18-19 & 2018 & aSP & Pisum sativum & no obvious symptoms & \\
\hline R4-18-20 & 2018 & aSP & Pisum sativum & no obvious symptoms & \\
\hline R4-18-21 & 2018 & sL & Trifolium pratense & no obvious symptoms & \\
\hline R4-18-22 & 2018 & $s L$ & Trifolium pratense & no obvious symptoms & \\
\hline$R 4-18-23$ & 2018 & $s L$ & Trifolium pratense & no obvious symptoms & \\
\hline R4-18-24 & 2018 & $s L$ & Trifolium repens & chlorotic spots & \\
\hline R4-18-25 & 2018 & $s L$ & Trifolium pratense & no obvious symptoms & \\
\hline R4-18-26 & 2018 & snL & Poacae sp. & no obvious symptoms & \\
\hline R4-18-27 & 2018 & snL & Chenopodium sp. & no obvious symptoms & \\
\hline R4-18-28 & 2018 & snL & Brassica napus & leaf reddening & \\
\hline R4-18-29 & 2018 & snL & $\begin{array}{l}\text { Matricaria } \\
\text { chamomilla }\end{array}$ & no obvious symptoms & \\
\hline R4-18-30 & 2018 & snL & Polygonaceae sp. & mottling & \\
\hline \multicolumn{6}{|c|}{ Landkreis Rostock (experimental field station, peas as green manure) } \\
\hline Sample & Season & Category & Plant & Symptoms & Temperature \\
\hline R5-16-01 & 2016 & SP & Pisum sativum & $\begin{array}{c}\text { top yellowing, pod deformation, } \\
\text { dwarfing }\end{array}$ & \multirow{10}{*}{$18^{\circ} \mathrm{C}$} \\
\hline R5-16-02 & 2016 & SP & Pisum sativum & top yellowing & \\
\hline R5-16-03 & 2016 & SP & Pisum sativum & top yellowing & \\
\hline R5-16-04 & 2016 & SP & Pisum sativum & top yellowing & \\
\hline R5-16-05 & 2016 & SP & Pisum sativum & top yellowing & \\
\hline R5-16-06 & 2016 & SP & Pisum sativum & severe yellowing & \\
\hline R5-16-07 & 2016 & SP & Pisum sativum & enation, vein clearing & \\
\hline R5-16-08 & 2016 & SP & Pisum sativum & top deformation & \\
\hline R5-16-09 & 2016 & SP & Pisum sativum & severe dwarfing & \\
\hline R5-16-10 & 2016 & SP & Pisum sativum & top yellowing & \\
\hline
\end{tabular}




\begin{tabular}{|c|c|c|c|c|c|}
\hline R5-16-11 & 2016 & aSP & Pisum sativum & no obvious symptoms & \\
\hline R5-16-12 & 2016 & aSP & Pisum sativum & no obvious symptoms & \\
\hline R5-16-13 & 2016 & aSP & Pisum sativum & no obvious symptoms & \\
\hline R5-16-14 & 2016 & aSP & Pisum sativum & no obvious symptoms & \\
\hline R5-16-15 & 2016 & aSP & Pisum sativum & no obvious symptoms & \\
\hline R5-16-16 & 2016 & aSP & Pisum sativum & no obvious symptoms & \\
\hline R5-16-17 & 2016 & aSP & Pisum sativum & no obvious symptoms & \\
\hline R5-16-18 & 2016 & aSP & Pisum sativum & no obvious symptoms & \\
\hline R5-16-19 & 2016 & aSP & Pisum sativum & no obvious symptoms & \\
\hline R5-16-20 & 2016 & aSP & Pisum sativum & no obvious symptoms & \\
\hline R5-16-21 & 2016 & $s L$ & Lupinus albus & no obvious symptoms & \\
\hline R5-16-22 & 2016 & $s L$ & Lupinus albus & no obvious symptoms & \\
\hline R5-16-23 & 2016 & $s L$ & $\begin{array}{c}\text { Lupinus } \\
\text { angustifolius }\end{array}$ & no obvious symptoms & \\
\hline R5-16-24 & 2016 & sL & $\begin{array}{c}\text { Lupinus } \\
\text { angustifolius }\end{array}$ & no obvious symptoms & \\
\hline R5-16-25 & 2016 & $s L$ & Lupinus albus & no obvious symptoms & \\
\hline R5-16-26 & 2016 & $\mathrm{snL}$ & Unknown & no obvious symptoms & \\
\hline R5-16-27 & 2016 & $\mathrm{snL}$ & Phacelia sp. & no obvious symptoms & \\
\hline R5-16-28 & 2016 & snL & Convolvulaceae sp. & no obvious symptoms & \\
\hline R5-16-29 & 2016 & $\mathrm{snL}$ & $\begin{array}{c}\text { Matricaria } \\
\text { chamomilla }\end{array}$ & no obvious symptoms & \\
\hline R5-16-30 & 2016 & snL & Chenopodium sp. & no obvious symptoms & \\
\hline R5-17-01 & 2017 & SP & Pisum sativum & dwarfing & \multirow{23}{*}{$16^{\circ} \mathrm{C}$} \\
\hline R5-17-02 & 2017 & SP & Pisum sativum & dwarfing & \\
\hline R5-17-03 & 2017 & SP & Pisum sativum & dwarfing & \\
\hline R5-17-04 & 2017 & SP & Pisum sativum & dwarfing & \\
\hline R5-17-05 & 2017 & SP & Pisum sativum & dwarfing & \\
\hline R5-17-06 & 2017 & SP & Pisum sativum & dwarfing & \\
\hline R5-17-07 & 2017 & SP & Pisum sativum & dwarfing & \\
\hline R5-17-08 & 2017 & SP & Pisum sativum & dwarfing & \\
\hline R5-17-09 & 2017 & SP & Pisum sativum & dwarfing & \\
\hline R5-17-10 & 2017 & SP & Pisum sativum & dwarfing & \\
\hline R5-17-11 & 2017 & aSP & Pisum sativum & no obvious symptoms & \\
\hline R5-17-12 & 2017 & aSP & Pisum sativum & no obvious symptoms & \\
\hline R5-17-13 & 2017 & aSP & Pisum sativum & no obvious symptoms & \\
\hline R5-17-14 & 2017 & aSP & Pisum sativum & no obvious symptoms & \\
\hline R5-17-15 & 2017 & aSP & Pisum sativum & no obvious symptoms & \\
\hline R5-17-16 & 2017 & aSP & Pisum sativum & no obvious symptoms & \\
\hline R5-17-17 & 2017 & aSP & Pisum sativum & no obvious symptoms & \\
\hline R5-17-18 & 2017 & aSP & Pisum sativum & no obvious symptoms & \\
\hline R5-17-19 & 2017 & aSP & Pisum sativum & no obvious symptoms & \\
\hline R5-17-20 & 2017 & aSP & Pisum sativum & no obvious symptoms & \\
\hline R5-17-21 & 2017 & $s L$ & Vicia faba & leaf deformation and rolling & \\
\hline R5-17-22 & 2017 & $s L$ & Trifolium sp. & no obvious symptoms & \\
\hline R5-17-23 & 2017 & $s L$ & Lupinus sp. & no obvious symptoms & \\
\hline
\end{tabular}




\begin{tabular}{|c|c|c|c|c|c|}
\hline R5-17-24 & 2017 & $s L$ & Vicia $s p$ & no obvious symptoms & \\
\hline R5-17-25 & 2017 & $s L$ & Trifolium sp. & no obvious symptoms & \\
\hline R5-17-26 & 2017 & snL & Borago officinalis & no obvious symptoms & \\
\hline R5-17-27 & 2017 & snL & Malva sp. & no obvious symptoms & \\
\hline R5-17-28 & 2017 & snL & Phacelia sp. & no obvious symptoms & \\
\hline R5-17-29 & 2017 & snL & Unknown & no obvious symptoms & \\
\hline R5-17-30 & 2017 & $\operatorname{snL}$ & $\begin{array}{c}\text { Matricaria } \\
\text { chamomilla }\end{array}$ & no obvious symptoms & \\
\hline R5-18-01 & 2018 & SP & Pisum sativum & mottling & \multirow{30}{*}{$20^{\circ} \mathrm{C}$} \\
\hline R5-18-02 & 2018 & SP & Pisum sativum & top yellowing & \\
\hline R5-18-03 & 2018 & SP & Pisum sativum & top yellowing & \\
\hline R5-18-04 & 2018 & SP & Pisum sativum & yellowing, mottling & \\
\hline R5-18-05 & 2018 & SP & Pisum sativum & yellowing, mottling & \\
\hline R5-18-06 & 2018 & SP & Pisum sativum & yellowing, mottling & \\
\hline R5-18-07 & 2018 & $\mathrm{SP}$ & Pisum sativum & yellowing, mottling & \\
\hline R5-18-08 & 2018 & SP & Pisum sativum & yellowing, mottling & \\
\hline R5-18-09 & 2018 & SP & Pisum sativum & yellowing, mottling & \\
\hline R5-18-10 & 2018 & SP & Pisum sativum & yellowing, mottling & \\
\hline R5-18-11 & 2018 & aSP & Pisum sativum & no obvious symptoms & \\
\hline R5-18-12 & 2018 & aSP & Pisum sativum & no obvious symptoms & \\
\hline R5-18-13 & 2018 & aSP & Pisum sativum & no obvious symptoms & \\
\hline R5-18-14 & 2018 & aSP & Pisum sativum & no obvious symptoms & \\
\hline R5-18-15 & 2018 & aSP & Pisum sativum & no obvious symptoms & \\
\hline R5-18-16 & 2018 & aSP & Pisum sativum & no obvious symptoms & \\
\hline R5-18-17 & 2018 & aSP & Pisum sativum & no obvious symptoms & \\
\hline R5-18-18 & 2018 & aSP & Pisum sativum & no obvious symptoms & \\
\hline R5-18-19 & 2018 & aSP & Pisum sativum & no obvious symptoms & \\
\hline R5-18-20 & 2018 & aSP & Pisum sativum & no obvious symptoms & \\
\hline R5-18-21 & 2018 & $s L$ & $\begin{array}{c}\text { Trifolium } \\
\text { incarnatum }\end{array}$ & chlorotic spots & \\
\hline R5-18-22 & 2018 & $s L$ & Trifolium repens & yellowing, mottling & \\
\hline R5-18-23 & 2018 & $s L$ & Vicia $s p$ & chlorotic spots & \\
\hline R5-18-24 & 2018 & $s L$ & Trifolium sp. & yellowing, mottling & \\
\hline R5-18-25 & 2018 & $s L$ & Trifolium repens & yellowing, mottling, leaf reddening & \\
\hline R5-18-26 & 2018 & snL & Chenopodium sp. & chlorotic spots & \\
\hline R5-18-27 & 2018 & snL & Solanum sp. & yellowing, mottling & \\
\hline R5-18-28 & 2018 & snL & Chenopodium sp. & leaf reddening, red ring spots & \\
\hline R5-18-29 & 2018 & snL & Brassicaceae sp. & yellowing, mottling & \\
\hline R5-18-30 & 2018 & snL & Unknown & no obvious symptoms & \\
\hline \multicolumn{6}{|c|}{ Landkreis Meißen (green pea production site) } \\
\hline Sample & Season & Category & Plant & Symptoms & Temperature \\
\hline R6-16-01 & 2016 & SP & Pisum sativum & yellowing & \multirow{4}{*}{$18^{\circ} \mathrm{C}$} \\
\hline R6-16-02 & 2016 & SP & Pisum sativum & enation & \\
\hline R6-16-03 & 2016 & SP & Pisum sativum & $\begin{array}{c}\text { mottling, mosaic, yellowing, vein } \\
\text { clearing }\end{array}$ & \\
\hline R6-16-04 & 2016 & SP & Pisum sativum & mottling, yellowing & \\
\hline
\end{tabular}




\begin{tabular}{|c|c|c|c|c|c|}
\hline R6-16-05 & 2016 & SP & Pisum sativum & yellowing, pod deformation & \\
\hline R6-16-06 & 2016 & SP & Pisum sativum & enation, pod deformation, mottling & \\
\hline R6-16-07 & 2016 & SP & Pisum sativum & enation, rusting & \\
\hline R6-16-08 & 2016 & SP & Pisum sativum & severe yellowing & \\
\hline R6-16-09 & 2016 & SP & Pisum sativum & $\begin{array}{l}\text { top dwarfing, leaf deformation, } \\
\text { yellowing, vein clearing, mottling }\end{array}$ & \\
\hline R6-16-10 & 2016 & SP & Pisum sativum & $\begin{array}{l}\text { severe yellowing, top deformation, } \\
\text { mottling }\end{array}$ & \\
\hline R6-16-11 & 2016 & aSP & Pisum sativum & no obvious symptoms & \\
\hline R6-16-12 & 2016 & aSP & Pisum sativum & no obvious symptoms & \\
\hline R6-16-13 & 2016 & aSP & Pisum sativum & no obvious symptoms & \\
\hline R6-16-14 & 2016 & aSP & Pisum sativum & no obvious symptoms & \\
\hline R6-16-15 & 2016 & aSP & Pisum sativum & no obvious symptoms & \\
\hline R6-16-16 & 2016 & aSP & Pisum sativum & no obvious symptoms & \\
\hline R6-16-17 & 2016 & aSP & Pisum sativum & no obvious symptoms & \\
\hline R6-16-18 & 2016 & aSP & Pisum sativum & no obvious symptoms & \\
\hline R6-16-19 & 2016 & aSP & Pisum sativum & no obvious symptoms & \\
\hline R6-16-20 & 2016 & aSP & Pisum sativum & no obvious symptoms & \\
\hline R6-16-21 & 2016 & $s L$ & Trifolium sp. & mottling & \\
\hline R6-16-22 & 2016 & $s L$ & Vicia $s p$ & no obvious symptoms & \\
\hline R6-16-23 & 2016 & $s L$ & Trifolium sp. & no obvious symptoms & \\
\hline R6-16-24 & 2016 & $s L$ & Vicia $s p$ & no obvious symptoms & \\
\hline R6-16-25 & 2016 & $s L$ & Vicia sp. & no obvious symptoms & \\
\hline R6-16-26 & 2016 & snL & Solanum sp. & no obvious symptoms & \\
\hline R6-16-27 & 2016 & snL & Poacae sp. & no obvious symptoms & \\
\hline R6-16-28 & 2016 & snL & Triticum sp. & no obvious symptoms & \\
\hline R6-16-29 & 2016 & snL & Brassicaceae sp. & no obvious symptoms & \\
\hline R6-16-30 & 2016 & snL & Unknown & no obvious symptoms & \\
\hline R6-17-01 & 2017 & SP & Pisum sativum & mosaic, mottling & \multirow{17}{*}{$18^{\circ} \mathrm{C}$} \\
\hline R6-17-02 & 2017 & SP & Pisum sativum & pod deformation & \\
\hline R6-17-03 & 2017 & SP & Pisum sativum & dwarfing & \\
\hline R6-17-04 & 2017 & SP & Pisum sativum & mottling, pod deformation, dwarfing & \\
\hline R6-17-05 & 2017 & SP & Pisum sativum & dwarfing & \\
\hline R6-17-06 & 2017 & SP & Pisum sativum & severe yellowing, mosaic & \\
\hline R6-17-07 & 2017 & SP & Pisum sativum & yellowing & \\
\hline R6-17-08 & 2017 & SP & Pisum sativum & yellowing, mottling & \\
\hline R6-17-09 & 2017 & SP & Pisum sativum & yellowing, mosaic, leaf rolling & \\
\hline R6-17-10 & 2017 & SP & Pisum sativum & pod deformation & \\
\hline R6-17-11 & 2017 & aSP & Pisum sativum & no obvious symptoms & \\
\hline R6-17-12 & 2017 & aSP & Pisum sativum & no obvious symptoms & \\
\hline R6-17-13 & 2017 & aSP & Pisum sativum & no obvious symptoms & \\
\hline R6-17-14 & 2017 & aSP & Pisum sativum & no obvious symptoms & \\
\hline R6-17-15 & 2017 & aSP & Pisum sativum & no obvious symptoms & \\
\hline R6-17-16 & 2017 & aSP & Pisum sativum & no obvious symptoms & \\
\hline R6-17-17 & 2017 & aSP & Pisum sativum & no obvious symptoms & \\
\hline
\end{tabular}




\begin{tabular}{|c|c|c|c|c|c|}
\hline R6-17-18 & 2017 & aSP & Pisum sativum & no obvious symptoms & \\
\hline R6-17-19 & 2017 & aSP & Pisum sativum & no obvious symptoms & \\
\hline R6-17-20 & 2017 & aSP & Pisum sativum & no obvious symptoms & \\
\hline R6-17-21 & 2017 & $s L$ & Trifolium repens & no obvious symptoms & \\
\hline R6-17-22 & 2017 & $s L$ & Trifolium sp. & no obvious symptoms & \\
\hline R6-17-23 & 2017 & $s L$ & Trifolium sp. & no obvious symptoms & \\
\hline R6-17-24 & 2017 & sL & Melilotus sp. & no obvious symptoms & \\
\hline R6-17-25 & 2017 & sL & Trifolium repens & no obvious symptoms & \\
\hline R6-17-26 & 2017 & snL & Chenopodium sp. & no obvious symptoms & \\
\hline R6-17-27 & 2017 & snL & Brassica napus & chlorotic spots, leaf deformation & \\
\hline R6-17-28 & 2017 & snL & Chenopodium sp. & no obvious symptoms & \\
\hline R6-17-29 & 2017 & snL & Poacae sp. & no obvious symptoms & \\
\hline R6-17-30 & 2017 & snL & Apiaceae sp. & no obvious symptoms & \\
\hline R6-18-01 & 2018 & SP & Pisum sativum & chlorosis & \multirow{30}{*}{$21^{\circ} \mathrm{C}$} \\
\hline R6-18-02 & 2018 & SP & Pisum sativum & dwarfing & \\
\hline R6-18-03 & 2018 & SP & Pisum sativum & chlorosis & \\
\hline R6-18-04 & 2018 & SP & Pisum sativum & chlorotic spots & \\
\hline R6-18-05 & 2018 & SP & Pisum sativum & chlorosis & \\
\hline R6-18-06 & 2018 & SP & Pisum sativum & chlorosis, mosaic & \\
\hline R6-18-07 & 2018 & SP & Pisum sativum & chlorosis, mosaic & \\
\hline R6-18-08 & 2018 & SP & Pisum sativum & chlorosis, leaf rolling & \\
\hline R6-18-09 & 2018 & SP & Pisum sativum & yellowing, dwarfing of side shoots & \\
\hline R6-18-10 & 2018 & SP & Pisum sativum & severe yellowing & \\
\hline R6-18-11 & 2018 & aSP & Pisum sativum & no obvious symptoms & \\
\hline R6-18-12 & 2018 & aSP & Pisum sativum & no obvious symptoms & \\
\hline R6-18-13 & 2018 & aSP & Pisum sativum & no obvious symptoms & \\
\hline R6-18-14 & 2018 & aSP & Pisum sativum & no obvious symptoms & \\
\hline R6-18-15 & 2018 & aSP & Pisum sativum & no obvious symptoms & \\
\hline R6-18-16 & 2018 & aSP & Pisum sativum & no obvious symptoms & \\
\hline R6-18-17 & 2018 & aSP & Pisum sativum & no obvious symptoms & \\
\hline R6-18-18 & 2018 & aSP & Pisum sativum & no obvious symptoms & \\
\hline R6-18-19 & 2018 & aSP & Pisum sativum & no obvious symptoms & \\
\hline R6-18-20 & 2018 & aSP & Pisum sativum & no obvious symptoms & \\
\hline R6-18-21 & 2018 & $s L$ & Polygonaceae sp. & no obvious symptoms & \\
\hline R6-18-22 & 2018 & sL & Polygonaceae sp. & no obvious symptoms & \\
\hline R6-18-23 & 2018 & $s L$ & Polygonaceae sp. & no obvious symptoms & \\
\hline$R 6-18-24$ & 2018 & $s L$ & Polygonaceae sp. & no obvious symptoms & \\
\hline R6-18-25 & 2018 & $s L$ & Polygonaceae sp. & no obvious symptoms & \\
\hline R6-18-26 & 2018 & $\mathrm{snL}$ & Poacae sp. & chlorotic spots, leaf reddening & \\
\hline R6-18-27 & 2018 & $\mathrm{snL}$ & Brassica napus & chlorotic spots & \\
\hline $\mathrm{R} 6-18-28$ & 2018 & snL & Helianthus annuus & chlorotic spots & \\
\hline R6-18-29 & 2018 & $\mathrm{snL}$ & Chenopodium sp. & chlorotic spots, mottling & \\
\hline R6-18-30 & 2018 & snL & Phacelia sp. & no obvious symptoms & \\
\hline
\end{tabular}

${ }^{*} \mathrm{NA}=$ no plants from the category were found at sampling. 
Table S2: List of primers used to confirm each virus detected by HTS.

\begin{tabular}{|c|c|c|c|c|}
\hline & \multirow{2}{*}{ Virus } & \multicolumn{3}{|c|}{ Primer } \\
\hline & & Name & Sequence & Reference \\
\hline \multirow{2}{*}{1} & \multirow{2}{*}{ Barley virus G } & HZ-714 & TGAGTCTCGCCAAACTCCAC & \multirow{2}{*}{ This article } \\
\hline & & $\mathrm{HZ}-715$ & GATTGGGATCCTCGTAGCGG & \\
\hline \multirow{2}{*}{2} & \multirow{2}{*}{$\begin{array}{l}\text { Bean common } \\
\text { mosaic virus }\end{array}$} & $\mathrm{HZ}-483$ & TGCAACATGGCACTTGAAGC & \multirow{2}{*}{ This article } \\
\hline & & $\mathrm{HZ}-484$ & ACGCATTCTGAGTGTGACGT & \\
\hline \multirow{2}{*}{3} & \multirow{2}{*}{$\begin{array}{c}\text { Bean leafroll } \\
\text { virus }\end{array}$} & S2 & ATCACITTCGGGCCGWSTCTATCAGA & \multirow{2}{*}{$\begin{array}{c}\text { (Abraham et al., } \\
\text { 2007) }\end{array}$} \\
\hline & & AS3 & CACGCGTCIACCTATTTIGGRTTITG & \\
\hline \multirow{2}{*}{4} & \multirow{2}{*}{$\begin{array}{l}\text { Bean yellow } \\
\text { mosaic virus }\end{array}$} & $\mathrm{HZ}-704$ & TGATGGATGTTGCGACAGCT & \multirow{2}{*}{ This article } \\
\hline & & $\mathrm{HZ}-705$ & GCCATTGCCGATCCAAATCC & \\
\hline \multirow{2}{*}{5} & \multirow{2}{*}{$\begin{array}{l}\text { Black grass } \\
\text { cryptic virus } 2\end{array}$} & $\mathrm{HZ}-791$ & CGCTGATTGGTCCGAATTCG & \multirow{2}{*}{ This article } \\
\hline & & $\mathrm{HZ}-792$ & AACGTCCCATTAGTGAGGCG & \\
\hline \multirow{2}{*}{6} & \multirow{2}{*}{$\begin{array}{c}\text { Carrot } \\
\text { torradovirus } 1\end{array}$} & $\mathrm{HZ}-682$ & TGCTAGCACACAAGGACAGG & \multirow{2}{*}{ This article } \\
\hline & & $\mathrm{HZ}-683$ & AGAGGCTGGGGAAAAAGTGG & \\
\hline \multirow{2}{*}{7} & \multirow{2}{*}{$\begin{array}{l}\text { Cherry leaf roll } \\
\text { virus }\end{array}$} & $\mathrm{HZ}-710$ & GCTGAATTGATGCGAGCCTG & \multirow{2}{*}{ This article } \\
\hline & & HZ-711 & TCGGGAGTGTCAATCCAAGC & \\
\hline \multirow{2}{*}{8} & \multirow{2}{*}{$\begin{array}{l}\text { Clover yellow } \\
\text { vein virus }\end{array}$} & $\mathrm{HZ}-702$ & CCGGGTTTAGTGACTCCAGC & \multirow{2}{*}{ This article } \\
\hline & & $\mathrm{HZ}-703$ & ACTAGCCCAGAGAGACGAGC & \\
\hline \multirow{2}{*}{9} & \multirow{2}{*}{$\begin{array}{l}\text { Cucumber } \\
\text { mosaic virus }\end{array}$} & CMV 5'CP & CTCGAATTCGGATCCGCTTCTCCGCGAG & 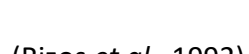 \\
\hline & & CMV 3'CP & GGCGAATTCGAGCTCGCCGTAAGCTGGATGGAC & (Rizos et al., 1992) \\
\hline 10 & Heracleum & HZ-696 & AGTTACTACCCCCAGAGCGT & \\
\hline 10 & latent virus & $\mathrm{HZ}-697$ & TGGGTACTTCGAATAGGCGC & IIIIS dictice \\
\hline 11 & Pea associatted & HZ-692 & TGGTCTCTTGCATGTTGCCT & Thin otiol o \\
\hline 11 & emaravirus & HZ-693 & TAAAGCAACCTCAGCTGGCA & Inis article \\
\hline & Pea associated & $\mathrm{HZ}-674$ & AGGTTTACCGCGATGGCTAG & \\
\hline 12 & mitovirus 1 & HZ-675 & CAGGGGCGTGAACAAGAGAT & Inis article \\
\hline & Pea associated & $\mathrm{HZ}-803$ & ACCGTCTTGTCTCAATCCCG & \\
\hline 13 & mitovirus 2 & $\mathrm{HZ}-804$ & TGACCTTTTTCGGGGCCAAT & Inis article \\
\hline & Pea associated & $\mathrm{HZ}-805$ & AAGCCGAATGAGGTGGGAAG & \\
\hline 14 & mitovirus 3 & $\mathrm{HZ}-806$ & GCATACGATCTGTTGCTGCG & This article \\
\hline & Pea enation & $\mathrm{HZ}-355$ & TCAGAAATGACGCCGGAACA & \\
\hline 15 & mosaic virus 1 & HZ-356 & GCGGAACAACCTGTCTCTGA & Chapter 4 \\
\hline & Pea enation & HZ-363 & GTTGTGCGTCCTCTTGGAGA & \\
\hline 16 & mosaic virus 2 & HZ-366 & CCCAAGGAGGTGTCCATGTC & Chapter 4 \\
\hline & Pea enation & HZ-698 & CCACGTTGAGATACCTCGCA & \\
\hline $1 /$ & $\begin{array}{l}\text { mosaic virus } \\
\text { satellite RNA }\end{array}$ & $\mathrm{HZ}-699$ & CCCCTGACACAATGCCATCT & Inis article \\
\hline & Pea necrotic & HZ-799 & TCTCTGGCGATACCCCTCTT & \\
\hline 18 & alphasatellite 1 & $\mathrm{HZ}-800$ & CCTCCACGCGTGTAGAAGAA & Inis article \\
\hline
\end{tabular}




\begin{tabular}{|c|c|c|c|c|}
\hline \multirow{2}{*}{19} & \multirow{2}{*}{$\begin{array}{c}\text { Pea necrotic } \\
\text { yellow dwarf } \\
\text { alphasatellite } 3\end{array}$} & HZ-801 & TTGTCCTTGGATACGCGTGT & \multirow{2}{*}{ This article } \\
\hline & & $\mathrm{HZ}-802$ & CTTCACCACCATTAGGGCCA & \\
\hline \multirow{2}{*}{20} & \multirow{2}{*}{$\begin{array}{c}\text { Pea necrotic } \\
\text { yellow dwarf } \\
\text { alphasatellite } 4\end{array}$} & $\mathrm{HZ}-740$ & TGAAAGAGCTCCTTCCAGGC & \multirow{2}{*}{ This article } \\
\hline & & HZ-741 & TGTCGATTTCCCTCCAGCTG & \\
\hline \multirow{2}{*}{21} & \multirow{2}{*}{$\begin{array}{c}\text { Pea necrotic } \\
\text { yellow dwarf } \\
\text { alphasatellite } 5\end{array}$} & $\mathrm{HZ}-742$ & TGGCGAGACGGTATTGTTTCA & \multirow{2}{*}{ This article } \\
\hline & & $\mathrm{HZ}-743$ & ATCTGGGTTTTGTCGCGGAT & \\
\hline \multirow{2}{*}{22} & \multirow{2}{*}{$\begin{array}{c}\text { Pea necrotic } \\
\text { yellow dwarf } \\
\text { alphasatellite } 6\end{array}$} & $\mathrm{HZ}-744$ & GGGCCAGAAGTCCAATATGCT & \multirow{2}{*}{ This article } \\
\hline & & $\mathrm{HZ}-745$ & TGCATCCGCCATTAGAGCTT & \\
\hline \multirow{2}{*}{23} & \multirow{2}{*}{$\begin{array}{c}\text { Pea necrotic } \\
\text { yellow dwarf } \\
\text { alphasatellite } 7\end{array}$} & $\mathrm{HZ}-824$ & ATGAAGATGGAGGACCCCGA & \multirow{2}{*}{ This article } \\
\hline & & HZ-825 & GCGCTACAGTTTGTCCGTTG & \\
\hline \multirow[b]{2}{*}{24} & \multirow{2}{*}{$\begin{array}{c}\text { Pea necrotic } \\
\text { yellow dwarf } \\
\text { virus }\end{array}$} & priPeaRdir & GGAATACCAAGGTGAGTTACGG & \multirow{2}{*}{ This article } \\
\hline & & priPeaRrev & TATTCCCTGAGAGTCCCGGAC & \\
\hline \multirow{2}{*}{25} & \multirow{2}{*}{$\begin{array}{l}\text { Pea seed-borne } \\
\text { mosaic virus }\end{array}$} & $\begin{array}{l}\text { PSbMV P1 } \\
\text { forward }\end{array}$ & GCTTCATGGTTGGAACTATTAAATG & \multirow{2}{*}{$\begin{array}{c}\text { (Giakountis et al. } \\
2015 \text { ) }\end{array}$} \\
\hline & & $\begin{array}{l}\text { PSbMV P1 } \\
\text { reverse }\end{array}$ & AAAGTTACTTGTTTTTGCATGCTTTC & \\
\hline \multirow{2}{*}{26} & \multirow{2}{*}{$\begin{array}{l}\text { Red clover } \\
\text { carlavirus } 1\end{array}$} & HZ-708 & ACAGCATGGGTGGGAATGAG & \multirow{2}{*}{ This article } \\
\hline & & HZ-709 & ACACTCCGTCGCGCTTATAG & \\
\hline \multirow{2}{*}{27} & \multirow{2}{*}{$\begin{array}{c}\text { Red clover } \\
\text { enamovirus } 1\end{array}$} & $\mathrm{HZ}-686$ & CGTTTTTCGGCTCTATGCAGC & \multirow{2}{*}{ This article } \\
\hline & & $\mathrm{HZ}-687$ & GGAGACTTTCTTGCCTCGCT & \\
\hline & Red clover & HZ-676 & GTCGCTGTCAGGAGTGGAAA & \\
\hline 28 & nepovirus $A$ & HZ-677 & CCGTCAAATTGTGCAGCACA & This article \\
\hline & Red clover & $\mathrm{HZ}-688$ & CTTTTGGTGTGCCAGGAACG & \\
\hline 29 & umbravirus & HZ-689 & TGATAGCAGAGGCAGGGACT & This article \\
\hline & Sclerotinia & $\mathrm{HZ}-690$ & AGCCGCCTTTACCATATCGC & \\
\hline 30 & $\begin{array}{c}\text { sclerotiorum } \\
\text { mitovirus } 4\end{array}$ & $\mathrm{HZ}-691$ & TGCTTCAGACACCATTCCTCC & This article \\
\hline 31 & sL associated & $\mathrm{HZ}-724$ & AGACGAGCTTCCCTGGTTTG & This article \\
\hline 31 & Ilarvirus & $\mathrm{HZ}-725$ & TTCCTCACACCACGCCTTAC & Inis article \\
\hline & snL associated & HZ-718 & TGGAGAGCATGACAGGCTTG & \\
\hline 32 & Chordovirus & HZ-719 & TTGACGGCCATCCAGAAAGT & This article \\
\hline & snL associated & $\mathrm{HZ}-730$ & CACCCAATAATGCCCCGACT & Thic articl \\
\hline 33 & Ilarvirus & $\mathrm{HZ}-731$ & GCGCAGTACTTCCCCTTCTT & Inis article \\
\hline & snL associated & $\mathrm{HZ}-694$ & AGCTGCACATCCGAAACTGA & This article \\
\hline 34 & Secoviridae & HZ-695 & CGCCATTTCAGCAAAACCCA & mins dicticte \\
\hline & snL associated & $\mathrm{HZ}-738$ & АTCTTGGAAGGCTGTGTCCC & Thic articlo \\
\hline 35 & Waikavirus & HZ-739 & AACAATGCCTGGCTCTAGCA & Inis article \\
\hline 36 & Soybean dwarf & SbDVf & GTCTACCTAAAAATTTTCAAAGAATCTG & (Abraham et al., \\
\hline 30 & virus & SbDVas & CGGACCCGGTTCTCCGTCTA & 2007) \\
\hline 37 & Soybean mosaic & $\mathrm{HZ}-493$ & GACAAGTGGGTTTGCGTTCC & This article \\
\hline 31 & virus & $\mathrm{HZ}-494$ & TAAGCCTGGATTTGCGCTCA & inis articie \\
\hline 38 & & $\mathrm{HZ}-716$ & ATTGCTCGCCATGAAGGACA & This article \\
\hline
\end{tabular}




\begin{tabular}{|c|c|c|c|c|}
\hline & $\begin{array}{l}\text { Turnip vein- } \\
\text { clearing virus }\end{array}$ & $\mathrm{HZ}-717$ & TGGGTGTAATTGAGCGTGCT & \\
\hline \multirow{2}{*}{39} & \multirow{2}{*}{$\begin{array}{l}\text { Turnip yellows } \\
\text { virus }\end{array}$} & $\mathrm{HZ}-809$ & ACCGTGGGTGGGTAGAAGAT & \multirow{2}{*}{ This article } \\
\hline & & $\mathrm{HZ}-810$ & ACTTTTCTGAACGCCCGGAT & \\
\hline \multirow{2}{*}{40} & \multirow{2}{*}{$\begin{array}{c}\text { Turnip yellows } \\
\text { virus associated } \\
\text { RNA }\end{array}$} & $\mathrm{HZ}-654$ & TGGACCGATACTTGCCCCTA & \multirow{2}{*}{ This article } \\
\hline & & $\mathrm{HZ}-655$ & AAGTGGGTATGCTGGAGTGC & \\
\hline \multirow{2}{*}{41} & \multirow{2}{*}{$\begin{array}{c}\text { Turnip yellows } \\
\text { virus associated } \\
\text { RNA } 2\end{array}$} & $\mathrm{HZ}-807$ & CCCGTCTGCTTCAAAGGACT & \multirow{2}{*}{ This article } \\
\hline & & $\mathrm{HZ}-808$ & CTCGTGGACCGGTTCTTCAA & \\
\hline \multirow{2}{*}{42} & \multirow{2}{*}{$\begin{array}{l}\text { White clover } \\
\text { cryptic virus } 2\end{array}$} & $\mathrm{HZ}-797$ & СCATCCCTGAAGATGCTGCT & \multirow{2}{*}{ This article } \\
\hline & & $\mathrm{HZ}-798$ & AGCGGAAGATAAGGCTGAGC & \\
\hline \multirow{2}{*}{43} & \multirow{2}{*}{$\begin{array}{l}\text { White clover } \\
\text { mosaic virus }\end{array}$} & $\mathrm{HZ}-793$ & TGATTGGTTACCAGTGGCCC & \multirow{2}{*}{ This article } \\
\hline & & $\mathrm{HZ}-794$ & GGTGTATTTCAGGGCACGGA & \\
\hline \multirow{2}{*}{44} & \multirow{2}{*}{$\begin{array}{l}\text { Wuhan insect } \\
\text { virus } 21\end{array}$} & $\mathrm{HZ}-732$ & AGATCGACGCGTCAGACATC & \multirow{2}{*}{ This article } \\
\hline & & $\mathrm{HZ}-733$ & TGGTTCCCTGTCGTACGTTG & \\
\hline
\end{tabular}


Table S3: Statistics of the raw data of the generated reads from each pool of the six German pea growing regions sampled over three seasons 2016, 2017 and 2018.

\begin{tabular}{|c|c|c|c|c|c|}
\hline Pool & Season & Raw reads & Quality >Q30 & $\begin{array}{l}\text { Quality } \\
\text { controlled reads }\end{array}$ & $\begin{array}{l}\text { Generated } \\
\text { contigs }\end{array}$ \\
\hline Region 1 & 2016 & 831076 & $90.0 \%$ & 689540 & 10457 \\
\hline Region 2 & 2016 & 814314 & $87.4 \%$ & 650346 & 18207 \\
\hline Region 3 & 2016 & 863642 & $89.6 \%$ & 700264 & 21660 \\
\hline Region 4 & 2016 & 900214 & $89.7 \%$ & 716702 & 21342 \\
\hline Region 5 & 2016 & 687850 & $89.0 \%$ & 502260 & 14335 \\
\hline Region 6 & 2016 & 885666 & $88.0 \%$ & 697544 & 16865 \\
\hline Symptomatic pea & 2016 & 931992 & $88.5 \%$ & 741390 & 16095 \\
\hline Asymptomatic pea & 2016 & 886412 & $88.0 \%$ & 699910 & 4258 \\
\hline surrounding legumes & 2016 & 1346156 & $87.4 \%$ & 1029344 & 23577 \\
\hline surrounding non-legumes & 2016 & 1102776 & $87.4 \%$ & 819312 & 33895 \\
\hline Region 1 & 2017 & 2032192 & $76.1 \%$ & 1103590 & 39489 \\
\hline Region 2 & 2017 & 2106164 & $76.5 \%$ & 1178880 & 30649 \\
\hline Region 3 & 2017 & 1616044 & $84.7 \%$ & 894074 & 38079 \\
\hline Region 4 & 2017 & 200210 & $59.3 \%$ & 91166 & 2297 \\
\hline Region 5 & 2017 & 2177380 & $78.2 \%$ & 1083988 & 47335 \\
\hline Region 6 & 2017 & 1123864 & $77.8 \%$ & 576750 & 24920 \\
\hline Symptomatic pea & 2017 & 1979682 & $79.0 \%$ & 1157966 & 36645 \\
\hline Asymptomatic pea & 2017 & 1931294 & $77.8 \%$ & 1045932 & 30680 \\
\hline surrounding legumes & 2017 & 1215960 & $82.2 \%$ & 684066 & 29305 \\
\hline surrounding non-legumes & 2017 & 1455624 & $77.7 \%$ & 754550 & 28433 \\
\hline Region 1 & 2018 & 1877930 & $84.2 \%$ & 815626 & 47637 \\
\hline Region 2 & 2018 & 2414122 & $86.8 \%$ & 1326580 & 67186 \\
\hline Region 3 & 2018 & 2511364 & $85.4 \%$ & 1354858 & 31197 \\
\hline Region 4 & 2018 & 3056130 & $83.9 \%$ & 1565932 & 113465 \\
\hline Region 5 & 2018 & 3404406 & $86.0 \%$ & 1794174 & 42290 \\
\hline Region 6 & 2018 & 2739384 & $84.7 \%$ & 1410600 & 31100 \\
\hline Symptomatic pea & 2018 & 2203570 & $88.4 \%$ & 1220234 & 80298 \\
\hline Asymptomatic pea & 2018 & 2125576 & $84.4 \%$ & 1264694 & 87403 \\
\hline surrounding legumes & 2018 & 2393184 & $88.9 \%$ & 1192422 & 11814 \\
\hline surrounding non-legumes & 2018 & 3299964 & $84.5 \%$ & 1832160 & 112211 \\
\hline
\end{tabular}


Table S4: List of the viruses and their associated nucleic acid satellites detected by HTS and confirmed by RT-PCR in each pool of the six German pea growing regions sampled over three seasons 2016, 2017 and 2018. 


\begin{tabular}{|c|c|c|c|c|c|c|c|c|c|c|c|c|c|c|c|c|c|c|c|c|c|c|}
\hline \multirow{2}{*}{ Virus } & \multirow{2}{*}{ Acronym } & \multirow{2}{*}{ Genus } & \multirow{2}{*}{ Family } & \multirow{2}{*}{\multicolumn{3}{|c|}{\begin{tabular}{|l|l|l|}
\multicolumn{3}{|c|}{ Salzlandkreis-1 } \\
2016 & 2017 & 2018 \\
\end{tabular}}} & \multicolumn{3}{|c|}{ Salzlandkreis-2 } & \multicolumn{3}{|c|}{$\begin{array}{l}\text { Münster } \\
\end{array}$} & \multicolumn{3}{|c|}{ Kreis Stormarn } & \multicolumn{3}{|c|}{ Landkreis Rostock } & \multicolumn{3}{|c|}{$\begin{array}{l}\text { Landkreis Meiißen } \\
\end{array}$} & \multirow{2}{*}{$\begin{array}{c}\text { Virus relative } \\
\text { abundance }\end{array}$} \\
\hline & & & & & & & 2016 & 2017 & 2018 & 2016 & 2017 & 2018 & 2016 & 2017 & 2018 & 2016 & 2017 & 2018 & 2016 & 2017 & 2018 & \\
\hline Barley virus $\mathrm{G}$ & BVG & $\begin{array}{l}\text { Polerovirus } \\
\text { Potwivucr }\end{array}$ & $\begin{array}{l}\text { Luteoviridae } \\
\text { Potwirider }\end{array}$ & & & & & & & & & & & & & & & & & & \begin{tabular}{|c|cl}
$\mathrm{snL}$ \\
\end{tabular} & \\
\hline $\begin{array}{l}\text { Bean common mosaic virus } \\
\text { Bean leafroll virus }\end{array}$ & BCMV & $\begin{array}{l}\text { Potyvirus } \\
\text { Luteopius } \\
\end{array}$ & $\begin{array}{l}\text { Potyviridae } \\
\text { Luteovirddee }\end{array}$ & & & & SP & $\frac{s L}{s p}$ & & & & & & & & & & & & & & 0.014 \\
\hline $\begin{array}{l}\text { Bean leadroll virus } \\
\text { Bean yellow mosaic virus }\end{array}$ & BYMV & Potyvirus & Potyviridae & & & SP & & & SP, aSP & & & & & & & & & & & & & 0.028 \\
\hline Black grass cryptic virus 2 & BGCV2 & & ssified virus & snt & & & & & & & & & & & & snt & snt & & & & & 0.014 \\
\hline Carrot torradovirus 1 & CaTV1 & Torradovirus & $\begin{array}{l}\text { Secoviridae } \\
\text { Sockidata }\end{array}$ & & & & & & & & snL & & & & & & & & & & & 0.014 \\
\hline 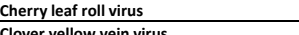 & $\frac{C L R V}{G(I Y Y V}$ & $\begin{array}{l}\text { Nepovirus } \\
\text { Potwirus }\end{array}$ & $\begin{array}{l}\text { Secoviridae } \\
\text { Pothwiride }\end{array}$ & & & snt & & & & & & & & & & & & & & & & 0.014 \\
\hline $\begin{array}{l}\text { Clover vellow veininivus } \\
\text { Cucumber mosaic virus }\end{array}$ & $\frac{\mathrm{CINV}}{\mathrm{CMV}}$ & $\begin{array}{l}\text { Potyvirus } \\
\text { Cucumovirus } \\
\end{array}$ & $\begin{array}{l}\text { Pottvirifae } \\
\text { Bromviridae }\end{array}$ & & & & & & $\frac{S P}{s L}$ & & $S P P$ & & & & & & & & & & & 0.028 \\
\hline Heracleum latent virus & HLV & Vitivirus & Betaflexiviridae & & & & & & & & $\mathrm{snL}$ & & & & & & & & & & & 0.014 \\
\hline Pea associated mitovirus 1 & PaMV1 & Mitovirus & Narnaviridae & & & & & & & & & & & & & & SP, asp & & & & & 0.028 \\
\hline $\begin{array}{l}\text { Pea associated mitovirus } 2 \\
\text { Pea associated mitovivus } 3\end{array}$ & $\begin{array}{l}\text { PaMV2 } \\
\text { PanY3 }\end{array}$ & $\begin{array}{l}\text { Mitovirus } \\
\text { Mitotirus }\end{array}$ & $\begin{array}{l}\text { Narnaviridae } \\
\text { Narraviride }\end{array}$ & & & & & & & & & & & & & & SP, aSP & & & & & $\frac{0.028}{0.014}$ \\
\hline $\begin{array}{l}\text { Peae associated mitovirus } 3 \\
\text { Pea associatted emaravirus }\end{array}$ & $\frac{\text { Palvs }}{\text { PaEV }}$ & $\begin{array}{l}\text { Emaravirus } \\
\end{array}$ & $\begin{array}{l}\text { Fimmoviridae } \\
\text { Fim }\end{array}$ & & & & & & & & & & & & & & & & & $S P$ & SP & 0.028 \\
\hline Pea enation mosaic virus 1 & PEMV1 & Enamovirus & Luteoviridae & $S P$, asp & SP, aSP & $S P$, asp & SP & SP, aSP & SP, asp & SP, asp & SP, asp & SP, aSP & SP, asp & SP, asp & & SP, aSP & & SP, aSP & SP, aSP & $S P, a S P$ & & 0.417 \\
\hline Pea enation mosaic virus 2 & PEMV2 & Umbravirus & Tombusviridae & SP, aSP & SP, aSP & Sp, asp & SP & $S P, a S^{P}$ & SP, asp & Sp, asp & SP, asp & SP, aSP & SP, asp & Sp, asP & & SP, aSP & & SP, aSP & Sp, asp & SP, aSP & $S P$, aSP & 0.444 \\
\hline Pea enation mosaic virus satellite RNA & PEMVsatRNA & Small linear sing & stranded RNA satellites & SP, aSP & SP, asP & & $\mathrm{sp}$ & $S p, a S P$ & SP, aSP & SP, aSP & SP, aSP & SP, aSP & SP, asp & & & SP, aSP & & & $S P$, asp & $S p, a s p$ & $S p$, asp & 0.361 \\
\hline 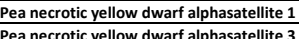 & $\begin{array}{ll}\text { PNYDVaSat1 } \\
\text { PNYYVagatz }\end{array}$ & & assatellite & & & 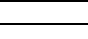 & $\mathrm{SP}$ & & & & & & $S P$ & & & 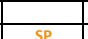 & & & & ( & SP & 0.028 \\
\hline 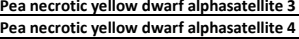 & $\begin{array}{l}\text { PNVYVasat3 } \\
\text { PNYVaSat4 }\end{array}$ & & $\begin{array}{l}\text { asastetille } \\
\text { asatelite }\end{array}$ & & $S P$ & & & & & & & & 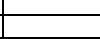 & & & $S P$ & & & & $S P$ & $S P$ & 0.022 \\
\hline Pea necrotic vellow dwarf alphasatellite 5 & PNYDVaSat5 & & asatellite & & SP & & & & SP & & & & & & & & & & & $S P$ & $S P$ & 0.056 \\
\hline Pea necrotic yellow dwarf alphasatellite 6 & PNYDVaSat6 & & asatellite & & $\mathrm{SP}$ & $\frac{S p}{50}$ & & & & & & & & & & & & & & & SP & 0.042 \\
\hline Pea necrotic yellow dwarf alphasatellite 7 & PNYDVaSat7 & & asatellite & & & $\mathrm{SP}$ & & & & & & & & & & & & & & & & 0.014 \\
\hline $\begin{array}{l}\text { Pea necrotic yellow dwarf virus } \\
\text { Pea seed-borne mosacicvirus }\end{array}$ & $\begin{array}{l}\text { PNYDV } \\
\text { PSbMV }\end{array}$ & $\frac{\text { Nanovirus }}{\text { Potvirus }}$ & $\begin{array}{l}\text { Nanoviridae } \\
\text { Potyviridae }\end{array}$ & $\mathrm{sP}$ & $\mathrm{SP}$ & $\mathrm{SP}$ & $\frac{S P}{S P}$ & SP, asp & $\frac{S P}{S P, a S P}$ & $S P P$ & & & $\frac{S p}{S P}$ & 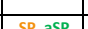 & $S P .>S P$ & $S P P$ & & & $S P P$ & $S P$ & SP & 0.153 \\
\hline $\begin{array}{l}\text { Pae seed-borne mosacic virus } \\
\text { Red clover carlavirus } 1\end{array}$ & $\frac{\text { PSONV }}{\text { RCCV1 }}$ & $\begin{array}{l}\text { Potylurus } \\
\text { Carlavius } \\
\end{array}$ & $\frac{\text { Postyviricae }}{\text { Betaflexiridae }}$ & & & & & & 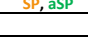 & & & & & 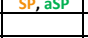 & 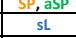 & & & & & & & $\frac{0.139}{0.014}$ \\
\hline $\begin{array}{l}\text { Ked cover caravirust } 1 \\
\text { Red clover enamovirus } 1 \\
\end{array}$ & & Enamovirus & Luteoviridae & & & & & & & sL. & & & & & 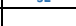 & & & & & SP & & 0.028 \\
\hline Red clover nepovirus $\mathrm{A}$ & $\begin{array}{lll}\text { RCNVA } & \end{array}$ & Nepovirus & Secoviridae & & & & & & & & & & & & & & sL & & & & & 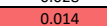 \\
\hline $\begin{array}{l}\frac{1}{\text { Red clover umbravirus }} \\
\end{array}$ & RCUV & Umbravirus & Tombusviridae & & & & & & & sL & & & & & & & 工 & & & SP & & 0.014 \\
\hline Sclerotinia sclerotiorum mitovirus 4 & SsMV4 & Mitovirus & Narnaviridae & & & & & & & & & & & & & & $S P$ & & & & & 0.014 \\
\hline sL associated llarvirus & sLalv & Mlarvirus & Bromoviridae & & & & & & & & & & & & & & & sL & & & & 0.014 \\
\hline snl associated Chordovirus & snlacy & Chorddovirus & Betaffexiviridae & & & & & & & & snt & & & & & & & & & & & 0.014 \\
\hline SnL associated Ilarvirus & snativ & Marvirus & Bromoviridae & & & & & & & & & & 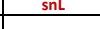 & & & & & & & & & 0.014 \\
\hline $\begin{array}{l}\text { SnL associated Secoviridae } \\
\text { sLL associated Waikairus }\end{array}$ & $\frac{\text { snlasv }}{\text { snlawV }}$ & Waikavirus & $\begin{array}{l}\text { Seccoviradae } \\
\text { Secoviridae }\end{array}$ & & & & & & & & sni & & & & snt & & & & & & & 0.014 \\
\hline Soybean dwarf virus & $\mathrm{SbDV}$ & Luteovirus & Luteoviridae & & & & & & & SP & & & sL & & sL & & & & & & & 0.044 \\
\hline Soybean mosaic virus & SMV & Pottvirus & Potyviridae & & & & & sL & & & & & & & & & & & & & & 0.014 \\
\hline Turnip vein-clearing virus & TVCV & Tobamovirus & Virgaviridae & & & & & & SnL & & & & & & & & & & & & & 0.014 \\
\hline Turnip yellows virus & TuYV & $\begin{array}{l}\text { Polerovirus } \\
\text { Unchassifics s.RR }\end{array}$ & $\begin{array}{l}\text { Lutueoviridace } \\
\text { nositive-strandyiruses }\end{array}$ & SP, aSP, SnL & SP, aSP, snL & $\frac{S P, a S p, s n L}{s, n}$ & $\frac{S P, \text { snL }}{S \mathrm{Dg}, \mathrm{gn}}$ & & $\frac{s p, a s p, \text { snl }}{s ! n}$ & $S P$, aSP, snL & SP, aSP, snL & SP, aSP, snL & SP, asp, snL & & & & & & $\begin{array}{ll}S P, a S P, \\
\text { SnLL }\end{array}$ & SP, asP, snL & $\mathrm{snL}$ & 0.472 \\
\hline $\begin{array}{l}\text { Turnip yellows sirus associated RNA } \\
\text { Trurip yelloww virus associated RNA }\end{array}$ & $\frac{\text { TuYVaRNA }}{\text { TuYVanNA2 }}$ & $\begin{array}{l}\text { Unclassified sRRn } \\
\text { Unclassified sRRn }\end{array}$ & $\begin{array}{l}\text { Positiviv-strand divuses } \\
\text { positiviv-strand diruses }\end{array}$ & & snnt & snt & $\frac{S P, \text { snL }}{S P}$ & & $\frac{\mathrm{snL}}{\mathrm{SP}, \mathrm{snL}}$ & & & & & & & & & & snt & & $\frac{s \mathrm{sn}}{\mathrm{sP \textrm {snL }}}$ & $\frac{0.083}{0.083}$ \\
\hline $\begin{array}{l}\text { White clover cryptic virus } 2 \\
\text { Wh }\end{array}$ & WCCV 2 & Betapartitivirus & 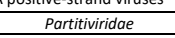 & & & & & & & & & sL & & sL & & & & & & & & 0.028 \\
\hline White clover mosaic virus & WCIMV & Potexvirus & Alphaflexiviridae & & & & sL & & & & & sL & & sL & sL & & & SL & & & & 0.069 \\
\hline Wuhan insect virus 21 & WHIV21 & & sified virus & & & & & & sL & & & & & & & & & & & & & 0.014 \\
\hline
\end{tabular}


Chapter 4: Comparative study on three viral enrichment procedures based on RNA extraction for plant viruses/viroids detection using high throughput sequencing

Yahya Zakaria Abdou Gaafar and H. Ziebell 


\subsection{Abstract}

High throughput sequencing (HTS) has become increasingly popular as virus diagnostic tool. It was used to detect and identify plant viruses and viroids in different types of samples. A virus sequence enrichment method prior to HTS is required to increase the viral reads in the generated data. In this study, we compared the sensitivity of three viral enrichment approaches i.e., double stranded RNA (dsRNA), ribosomal RNA depleted total RNA (ribo-depleted totRNA) and small RNA (sRNA) for plant virus/viroid detection. The three viral enrichment approaches used here enabled the detection of all known viruses/viroid, in the study, with different amounts of viral/viroid nucleotides and depths. Interestingly, both dsRNA and ribo-depleted totRNA approaches detected a divergent strain of Wuhan aphid virus 2 as well. Moreover, Vicia cryptic virus was detected in the data of dsRNA and sRNA approaches only. These results support the ability of HTS for the detection of plant viruses/viroids using RNA extracts from different plant samples. The dsRNA approach used here detected all viruses/viroid, consumed less time, is lower in cost, and required less starting material. This study can serve as guidelines for starting virus diagnostics laboratories.

\subsection{Introduction}

Viruses and viroids are one of the major emerging threats to agricultural and horticultural production (Anderson et al., 2004). Climate change and increasing global trade are only two factors accelerating the dispersal of plant viruses by vectors into new geographic areas where they can potentially cause greater damage thus threating food supplying for humans and animals (Canto et al., 2009; Hulme, 2017; Jones, 2016; Myers et al., 2017). Correct identification of the underlaying causes of the diseases is important for correct management procedures, such as switching to virus-resistant cultivars (where available), quarantine or eradication measures or vector control. In the past, traditional detection methods such as serological (i.e., ELISA, Tissue blot-ELISA) or nucleic acid-based (PCR, probe-based methods) detection methods required priori knowledge of the pathogen that needed to be detected, such as previously purified virus particles used for raising antibodies or nucleic acid sequences for the design of specific primers or target probes (Ward et al., 2004). However, divergent sequences or virus variants with different antigenic epitopes on the virion surface would not be detected using these methods. Additionally, using electron microscopy often failed to detect low titre viruses or viruses that are phloem-based; disease-causing entities without protein shells such as viroids or satellite RNAs would not be detected at all.

The development and evolution of novel high throughput sequencing (HTS) technologies has revolutionised virus discovery, plant virus diagnostics as well as metagenomic, evolutionary and community studies in recent years (Roossinck, 2017; Villamor et al., 2019). Unlike the methods described above, no prior knowledge about the pathogen is needed for HTS since all the nucleic acid (viral or non-viral) in the sample can 
be sequenced. Many new viruses and viroids have been discovered using HTS and the number is increasing (Elbeaino et al., 2015; Chen et al., 2016; Villamor et al., 2017). However, HTS-based approaches for virus/viroid detection is facing several challenges in order to be validated for routine diagnostic laboratories (Maree et al., 2018). As HTS can sequence all nucleic acids within the given sample, no matter if of plant or pathogen origin, suitable enrichment strategies should be used to minimise the generated host reads that reduces the pathogen reads and may interfere with subsequent bioinformatic analyses. Additionally, there is no universal sequence that can be used for the analysis of virus/viroid communities in contrast to fungi or bacteria where the internal transcribed spacer (ITS) or 16S ribosomal RNA can be used in amplicon sequencing manner for the general detection of these pathogens (Leff et al., 2017). Thus, in case of unknown virus/viroid infections, studies relay on untargeted identification approaches utilizing random primers for detection (Gaafar et al., 2018b).

As viruses/viroids have different genome types i.e., single- or double-stranded DNA, single- (positive [+ve] or negative [-ve]) or double-stranded RNA, circular or noncircular nucleic acids, there are various extraction methods and enrichment strategies available for these molecules. For example, the extraction of dsRNA (the replicative form of most plant viruses) has been used for a long time to generate sequence information from plant viruses and can be used for the enrichment of viral sequences for HTS (Morris, 1979; Bar-Joseph et al., 1983; Roossinck et al., 2010; Gaafar et al., 2019c).

More recently, virus-derived small RNAs (vsRNAs) or ribo-depleted total RNA extracts have been used to prepare samples for HTS (Kreuze et al., 2009; Gaafar et al., 2019d). Alternatively, rolling circle amplification and subsequent sequencing worked well for viruses with circular DNA genomes (Wyant et al., 2012; Gaafar et al., 2018a). Few studies have directly compared different enrichment strategies and their ability to detect plant virus sequences through HTS (Visser et al., 2016; Pecman et al., 2017). In this study, we compare the virus/viroid detection, their genome coverage recovery and depth from the reads produced using three RNA based-enrichment strategies, i.e., dsRNA extraction, ribo-depleted totRNA and SRNA extraction. We included viruses with different genomes ((+ve) ssRNA, (-ve) ssRNA and ssDNA) as well as a viroid. 


\subsection{Material and methods}

\subsubsection{Plant cultivation}

Four plant species (Nicotiana benthamiana [cultivar: JKI-Wild], Pisum sativum [cultivar: Rainier], Solanum lycopersicum [cultivar: Linda] and Vicia faba [cultivar: Tattoo]) were used (Table 1). In addition, Phaseolus vulgaris (cultivar: Black Turtle) seed infected with the cryptic virus phaseolus vulgaris alphaendornavirus 1 and 2 (PVEV1 and PvEV2 JKI ID 31403) were also sown to spike the samples during extraction (kindly provided by Dr. Mike Rott). The plants were kept under greenhouse conditions (at $22^{\circ} \mathrm{C}$; photoperiod of $16 \mathrm{~h}$ light [natural daylight with additional growth light Phillips IP65, 400 Watt] and $8 \mathrm{~h}$ dark). 


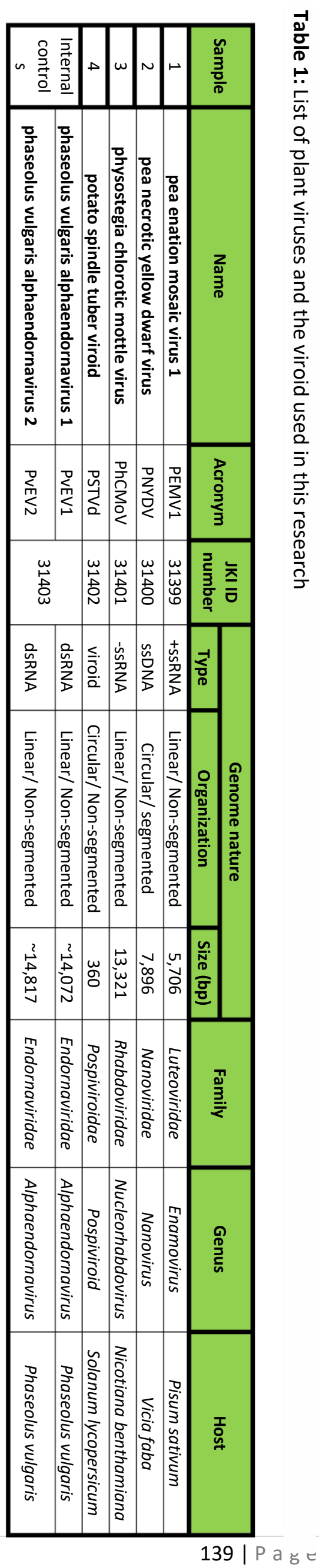

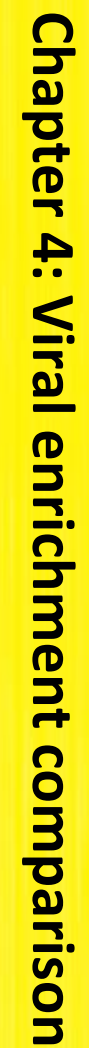




\subsubsection{Viruses and viroid isolates}

Four viruses with different genomes and one viroid were used in this research (Table 1). Pea enation mosaic virus 1 (PEMV1) originally from a $P$. sativum plant showing enation symptoms, collected in 2011 from Hondeghem, northern France. Pea necrotic yellow dwarf virus (PNYDV), Elbtal isolate, originally from infected $P$. sativum sample showing top leaves dwarfing and yellowing and leaf rolling symptoms, from Saxony, Germany in 2011. The original physostegia chlorotic mottle virus (PhCMoV), HZ16-558 isolate, was from infected S. lycopersicum plant, collected from Hesse state in Germany with fruits marbling and discoloration symptoms in 2016 (Gaafar et al., 2018a). The potato spindle tuber viroid (PSTVd), isolate PV-0950, was kindly provided by DSMZ (German collection of microorganisms and cell cultures) in the form of lyophilized infected $S$. lycopersicum plant leaves in 2014.

\subsubsection{Virus maintenance}

PEMV1 and PNYDV were maintained by aphid transmission using Acyrthosiphon pisum. The aphids were reared for five days on infected $P$. sativum and $V$. faba, respectively, and ten viruliferous aphids were transferred onto healthy plants. The inoculation access period was five days. The aphids were killed using a non-systemic insecticide (Spruzit Schädlingsfrei, Neudorff GmbH KG, Germany).

PhCMoV and PSTVd were maintained by mechanical transmission to $N$. benthamiana and S. lycopersicum, respectively. For mechanical transmission, $100 \mathrm{mg}$ of infected plant material was ground in Norit buffer (0.05M phosphate buffer [pH 7.0]; $0.001 \mathrm{M}$ ethylenediaminetetraacetic acid; $0.02 \mathrm{M}$ sodium diethyldithiocarbamic acid; $0.005 \mathrm{M}$ thioglycolic acid; $0.75 \%$ activated charcoal [Norit]) and $30 \mathrm{mg}$ of diatomaceous earth (Celite) was added. The homogenate was rubbed gently on healthy plants' leaves using glass rods. The inoculated leaves were rinsed with water within 5 min. After inoculation, all plants were kept under greenhouse conditions for four weeks until virus symptoms were observed (except for PSTVd; no symptoms).

\subsubsection{Confirmation of infection by DAS-ELISA and/or PCR/RT-PCR}

To confirm infection, all plants were tested by ELISA and/or RT-PCR or PCR (Table 2), ELISA tests were performed, except for PSTVd infected plants, using antibodies mentioned in Table 2 as described in (Clark \& Adams, 1977; Fletcher et al., 2016). Additionally, to confirm infections with PEMV1, PEMV2, PhCMoV and PSTVd, RT-PCR was performed using total RNA extracted with innuPREP Plant RNA Kit (Analytik Jena, Germany), following the manufacturer's instructions. cDNA was synthesised using ProtoScript II Reverse Transcriptase (New England Biolabs) using the reverse primer of the primer pairs mentioned for each virus (Table 2). PNYDV infection was confirmed by DNA extraction according to Edward's method for plant DNA extraction with $0.1 \%$ 
Mercaptoethanol added to the extraction buffer followed by PCR using PNYDV specific primers (Edwards et al., 1991). 


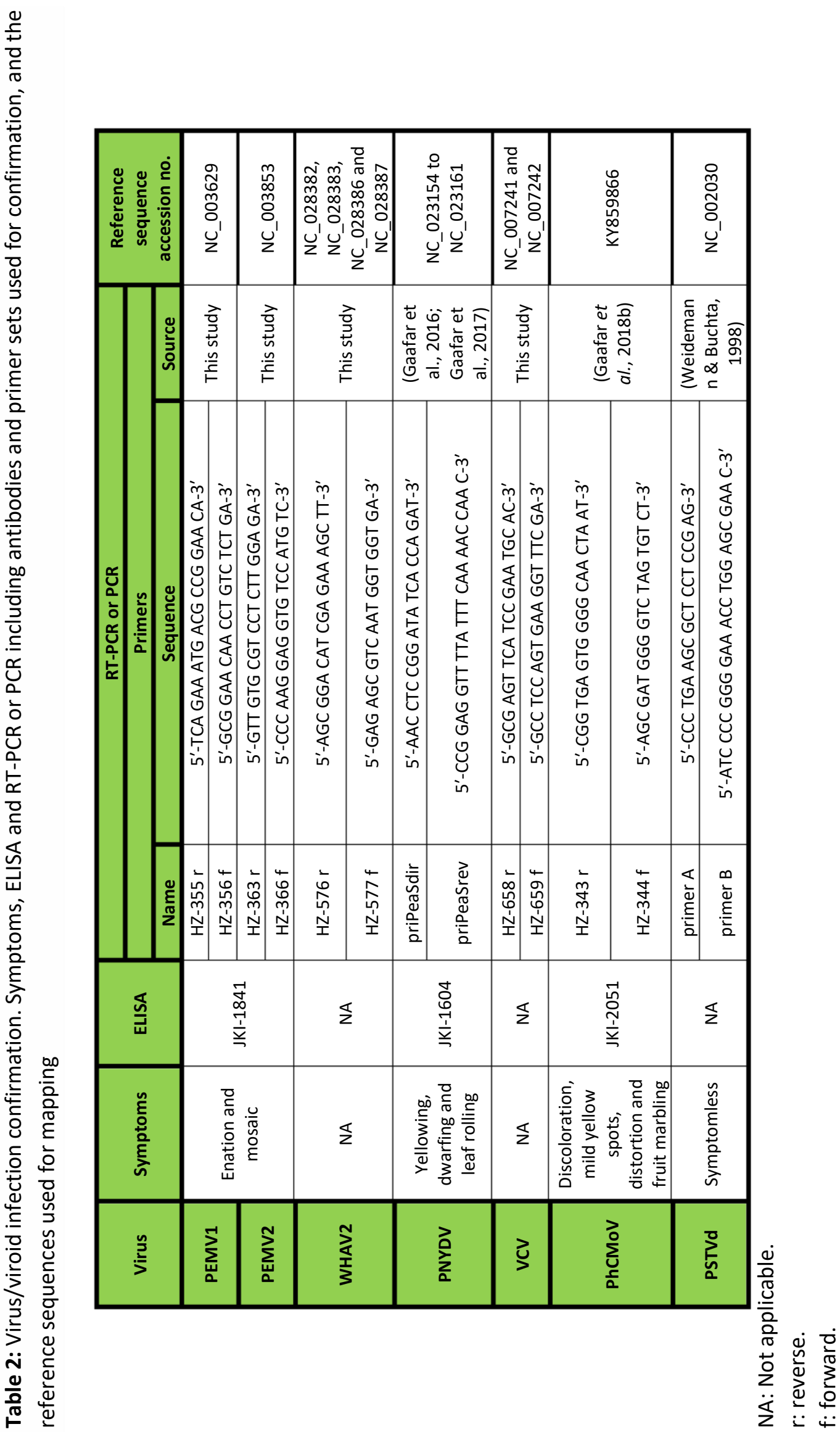




\subsubsection{Nucleic acid extraction and virus/viroid enrichment}

Five grams of leaf tissue from each infected plant were ground in liquid nitrogen and stored at $-80^{\circ} \mathrm{C}$ until further extraction. For extraction, $100 \mathrm{mg}$ leaf materials were mixed 20 mg leaf disc from $P$. vulgaris infected with PvEV1 and PVEV2. The mix was used for three different RNA extraction methods (Fig 1):

a) Double-stranded RNA extraction (dsRNA):

dsRNA was extracted using Double-RNA Viral dsRNA Extraction Mini Kit (iNtRON Biotechnology, USA) according the manufacturer's protocol.

b) Total RNA extraction followed by ribo-depletion (ribo-depleted totRNA):

Total RNA extraction was performed using innuPREP Plant RNA Kit as described by the manufacturers' instructions. The ribosomal RNA (rRNA) was depleted using the RiboMinus ${ }^{\mathrm{TM}}$ Plant Kit for RNA-Seq (Invitrogen) according the manufacturers' protocol.

c) Total RNA extraction followed by small RNA extraction (sRNA):

Total RNA was extracted as described above, then DNase treated using innuPREP DNase I Digest Kit (Analytik Jena AG) according the manufacturers' protocol. sRNA was extracted using polyacrylamide gel selection at Fasteris Life Sciences SA (Plan-les-Ouates, Switzerland).

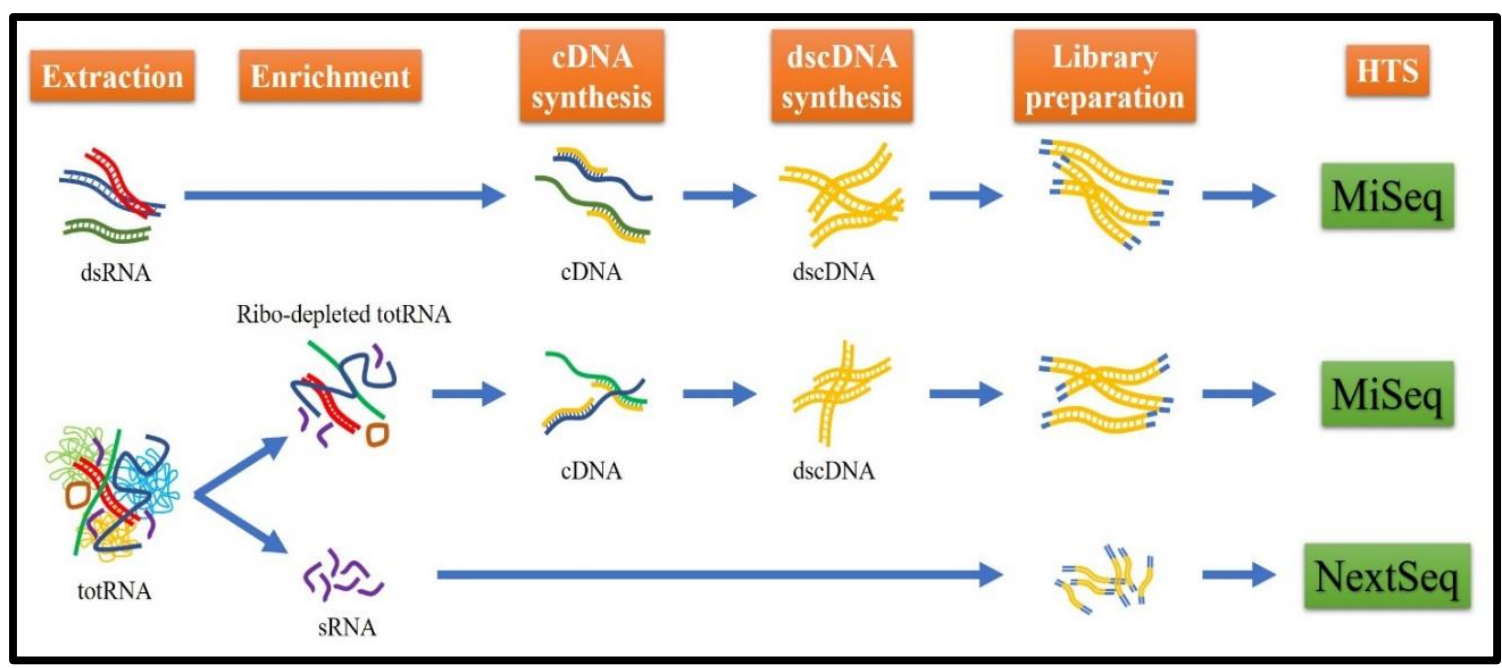

Figure 1: Graphical representation of the three RNA approaches used in this research i.e., dsRNA, ribo-depleted totRNA and SRNA. The steps are mentioned in orange boxes. The sequencing Illumina platforms are in green.

Additionally, DNA extraction followed by rolling circle amplification (RCA) was carried out for the nanovirus infected plants. Genomic DNA was extracted as described 
before. The extracted DNA was treated by RNase A followed by RCA using TempliPhiTM 100 Amplification Kit (GE Healthcare Limited, UK).

\subsubsection{Nucleic acid preparations for HTS}

For dsRNA and ribo-depleted totRNA, random cDNA was synthesized using ProtoScript II Reverse Transcriptase and random octamer primers (8N). A denaturation step of $99^{\circ} \mathrm{C}$ for $2 \mathrm{~min}$ for the dsRNA and $65^{\circ} \mathrm{C}$ for $5 \mathrm{~min}$ for the ribo-depleted totRNA. dscDNA was synthesized using NEBNext Ultra II Non-Directional RNA Second Strand Synthesis Module (New England Biolabs). Libraries were prepared using Nextera DNA Library Prep Kit (Illumina) following the manufacturer protocol. The quantification was done using Qubit dsDNA HS Assay Kit (Life Technologies) and quality analysis was done using High Sensitivity DNA Chips on Agilent 2100 Bioanalyzer (Agilent Technologies) following the manufacturers' protocols. Subsequently, the libraries were sequenced on a MiSeq Illumine platform v.3 pair-end reads $(2 \times 301)$ at DSMZ, Germany. For the sRNA, libraries were prepared from sRNA extracted using TruSeq small RNA kit (Illumina) at Fasteris Life Sciences SA (Plan-les-Ouates, Switzerland) and sequenced on a NextSeq Illumine platform single-end reads (1x50). For the RCA products, the library was also prepared using Nextera DNA Library Prep Kit and run on a NextSeq Illumine platform $(2 \times 151)$ at DSMZ.

\subsubsection{Bioinformatic data analysis}

The data analysis was performed using Geneious (version 11.1.5) (Biomatters Limited, Auckland, New Zealand). The adaptors and low-quality nucleotides were trimmed from the raw reads (quality score set to 0.05 ), then the trimmed reads were filtered by length (100 to $301 \mathrm{nt}$ for dsRNA and ribo-depleted totRNA; 20 to $24 \mathrm{nt}$ for sRNA). The filtered trimmed reads were de novo assembled using Geneious (parameters; Medium Sensitivity/Fast). Moreover, sRNA reads were also assembled using Velvet ( $k m e r=13$ / minimum contig length $=30$ ) (Zerbino \& Birney, 2008).

The filtered-quality trimmed reads were also kmer normalised using BBNorm tool 37.64 (Brian Bushnell within Geneious) (parameters: Minimum depth $=5 /$ Target coverage level $=40$ for MiSeq reads, and Minimum depth $=5 /$ Target coverage level $=100$ for NextSeq reads). After that, the reads were de novo assembled as described above.

Assembled contigs were compared against a local database for viruses and viroids reference sequences using BLASTn (maximum E-value: 1e-5) downloaded 18 August 2018. To confirm the virus/viroid presence in each sample, the contigs were mapped to references (accession no. in Table 2). Additionally, filtered and trimmed reads were mapped to the reference sequences (Geneious; Medium Sensitivity/Fast and 5 iterations for dsRNA and ribo-depleted totRNA, and Medium-low Sensitivity/Fast and 5 iterations for SRNA). A cut-off for virus/viroid detection was set at $\geq 40 \%$ recovery of the reference sequence (for viruses) and $\geq 80 \%$ (for viroids). The consensus sequences were generated 
from the quality trimmed reads by mapping to reference sequences. The results were manually inspected to refine the ends of the genomes and consensus sequences were generated based on the quality of the nucleotides.

Pairwise nucleotide alignments were performed with ClustalW 2.1 (Cost matrix: CLUSTALW/ Gap open cost: 15/ Gap extend cost: 6.66) on Geneious. While for protein alignments Clustal W 2.1 with parameters (Cost matrix: BLOSUM/ Gap open cost: 10/ Gap extend cost: 0.1 ).

\subsubsection{Comparing the three RNA-based approaches}

The quality-controlled reads of each dataset were randomly subsampled (10 replicates) into the same number of reads (equal to approximately the same number of nucleotides 1, 10, 20, 30, 40 and 50 million nt). Resulting in a total of 720 subsets, each was used for de novo assembly and mapping to its reciprocal consensus sequence generated from the total reads. The number of nucleotides matched the references, percentage of the reference sequence recovered, and mean depth were calculated for each. Furthermore, de novo assembly (Geneious parameters; Medium Sensitivity/Fast) was performed for each subset and the resulting contigs were mapped to the corresponding consensus virus/viroid sequence and the percentages of whole genome was generated.

\subsubsection{Statistical analysis}

The generated data from the bioinformatic analysis was statistically analysed using $R$ version 3.5.1 ( $R$ Core Team, 2014). The number of nucleotides matched the references, percentage of the reference sequence recovered by both reads and de novo assembled contigs, and mean depth were statistically compared. The data were visualized by ggplot2 and VennDiagram packages (Chen \& Boutros, 2011; Ginestet, 2011).

\subsection{Results}

\subsubsection{Raw data}

The statistics of the raw data generated from the HTS platforms of the three RNAbased approaches are mentioned in S1 Table. As we used restricted quality and length of the reads, part of the generated datasets was less quality than expected, these can be shown by the number of reads after trimming and filtering and by the mean read length. Nevertheless, the datasets were still used for bioinformatic analysis. Furthermore, the spiked internal control viruses PVEV1 and PVEV2 were detected in all the samples with all the different approaches in different amounts of reads and depths (S2 Table).

The costs of each approach on were calculated on average based on our experience and the prices until early 2019 (S3 Table). The average cost of the dsRNA 
approach was about 307 Euros, the ribo-depleted totRNA cost 380 Euro, while sRNA approach cost about 348 Euro on average.

\subsubsection{Virus/viroid detection}

Using BLASTn search, all three known viruses and the viroid in this study were detected with all three RNA approaches i.e., dsRNA, ribo-depleted totRNA and sRNA. Surprisingly, other viruses were detected in sample 1 and sample 2 . In sample 1 , in addition to PEMV1, a divergent isolate of pea enation mosaic virus 2 (PEMV2) (Genus: Umbravirus/ Family: Tombusviridae) was detected using all three approaches, and a divergent strain of Wuhan aphid virus 2 (WHAV2) was detected by dsRNA and ribodepleted totRNA approaches (Fig 2). In sample 2, in addition to PNYDV, Vicia cryptic virus (VCV) (Genus: Alphacryptovirus/ Family: Partitiviridae) was also detected by dsRNA and sRNA approaches (Fig 2). These results showed that all viruses (Known and unknown) were detected by the dsRNA approach while ribo-depleted RNA and sRNA approaches detected either WHAV2 or VCV, respectively. No associated RNAs or alphasatellites DNAs were identified in the different samples. The presence of PEMV2, WHAV2 and VCV were confirmed using RT-PCR and virus specific primers as listed in Table 2.

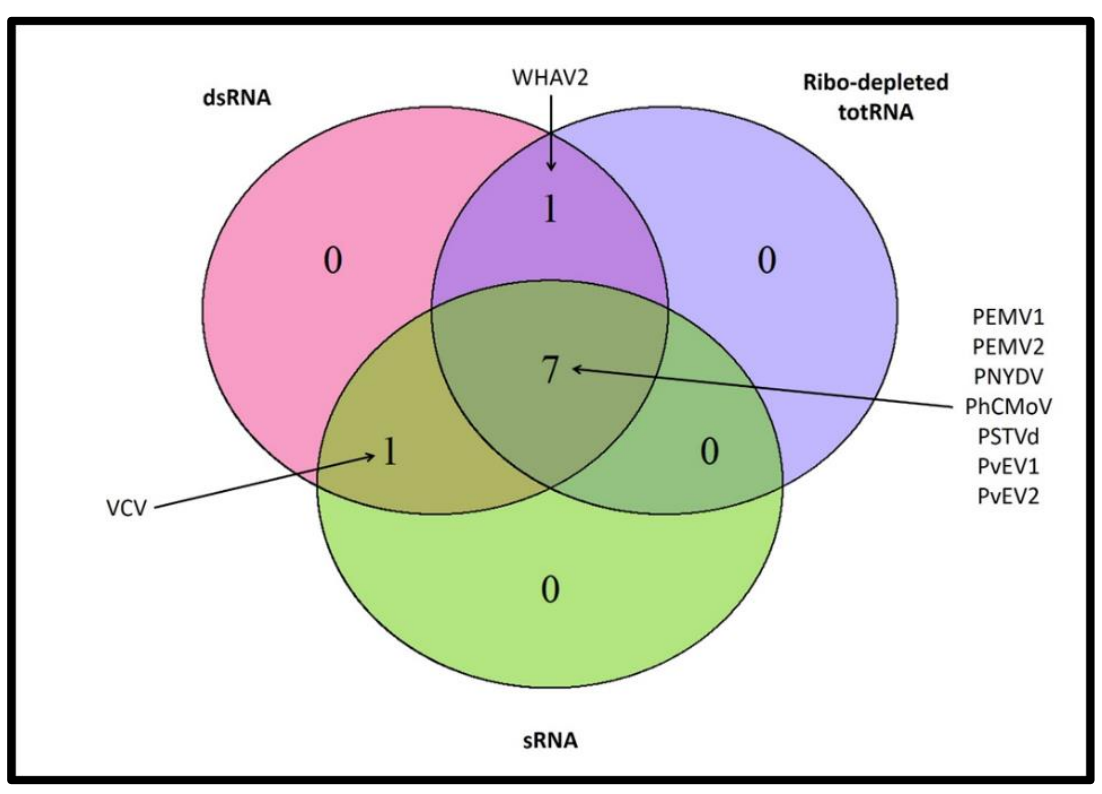

Figure 2: Venn diagram showing the viruses/viroid detected in all samples using different viral enrichment approaches (dsRNA, ribo-depleted totRNA and SRNA). The overlapping regions correspond to the number of viruses/viroid detected by more than one approach. The detected viruses were PEMV1: pea enation mosaic virus 1, PEMV2: pea enation mosaic virus 2, WHAV2: Wuhan aphid virus 2, PNYDV: pea necrotic yellow dwarf virus, VCV: Vicia cryptic virus and PhCMoV: physostegia chlorotic mottle virus, PSTVd: potato spindle tuber viroid, PVEV1: phaseolus vulgaris alphaendornavirus 1, and PvEV2: phaseolus vulgaris alphaendornavirus 2. 


\subsubsection{Virus/viroid recovery}

The total filter and trimmed reads of each dataset were mapped to the different reference genome of the nine viruses in the samples (six viruses, a viroid and the two spiked internal control viruses). The total mapped reads and the percentage of the reference coverage can be found in Table 3. The numbers of mapped reads are different from one approach to another and from one virus to another. The full genomes of PEMV2, $\mathrm{PhCMOV}$ and PSTVd was recovered in the datasets by all three viral enrichment approaches. While the almost complete genomes of PEMV1 (missing the ends [not confirmed]) was detected by the three approaches. The almost complete genome of WHAV2 (missing the ends of some segments [not confirmed]) was identified by both dsRNA and ribo-depleted totRNA approaches. For VCV, less than $90 \%$ of the genome was assembled by ribo-depleted totRNA and sRNA approaches, but for PNYDV, less than $80 \%$ of the genome was assembled by the three approaches (Table 3). Therefore, to obtain the full genome of PNYDV, RCA enrichment was used. All virus sequences were submitted to GenBank (accession no. MK948524 to MK948543). 


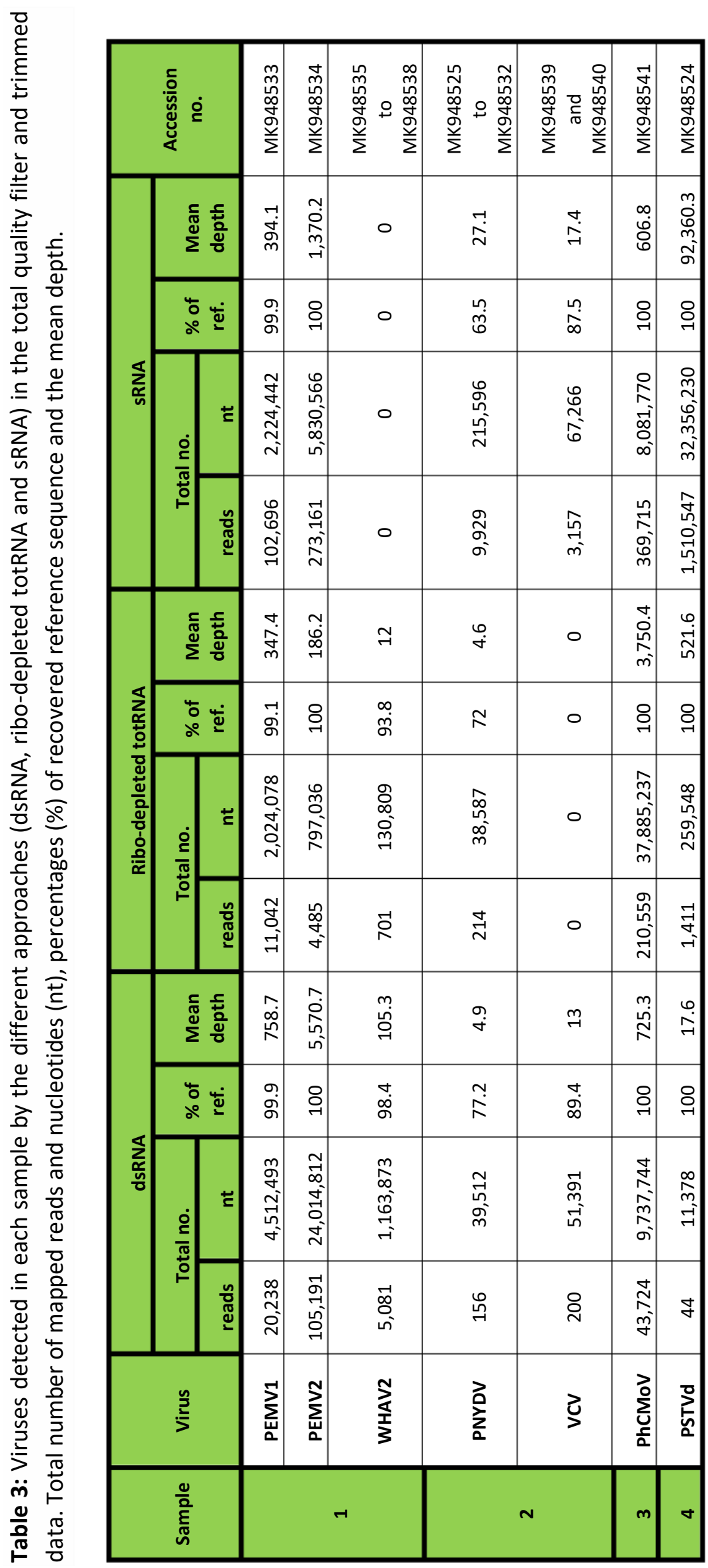




\subsubsection{Virus/viroid sequences characterisation}

Pairwise nucleotide comparison between the sequences of PEMV1, PNYDV, PhCMoV and PSTVd showed nt identities range between $95.7 \%$ and $100 \%$ to their closest sequences. The French isolates of PEMV1 resulted in $95.7 \%$ identity with the ID isolate from Idaho USA (accession no. HM439775). The 8 segments of PNYDV Elbtal isolate shared between $97.9 \%$ to $99.9 \% \mathrm{nt}$ identities to their closest sequences on NCBI. Segments DNA$\mathrm{N},-\mathrm{R},-\mathrm{S},-\mathrm{U} 4$ shared $99.6 \%$ to $99.9 \%$ nt identities to the German isolate 110726 (accession no. KY810776 to KY810778 and KY810781). While segments DNA-C, -U1 and -U2 were close to the Danish isolate DK HZ16-582 by $97.9 \%$ to $99.4 \%$ (accession no. MH000257, MH000258 and MH000260), and segment DNA-M is closer to the Danish isolate DK HZ16573 by $98.6 \%$ (accession no. MH000250). PhCMoV HZ16-558 isolate shared $99.6 \%$ nt identity to HZ15-192 isolate (accession no. KY859866). The PSTVd isolate shared $100 \%$ nt identity with isolates 6718566 from Netherlands and 07087900 from Belgium (accession no. KX370618 and FM998548, respectively). The sequences of PEMV2 and WHAV2 isolates were divergent from the reference sequences (S4 and S5 Tables). PEMV2 predicted proteins shared between $92.5 \%$ to $97.6 \%$ aa identities to their analogues of the closest isolate from the UK (S4 Table) whereas WHAV2 proteins shared $90.2 \%$ to $96.8 \%$ aa identities to the Chinese strain WHYC-2 predicted proteins (S5 Table). PVEV1 shared $99.7 \%$ nt identity to the Mexican isolate INIFAP CG1 (accession no. MG640415) and PvEV2 shared closest nt identity with a Brazilian isolate with 99.4\% (accession no. AB719398).

\subsubsection{The sensitivity of the three approaches (virus nucleotides and average depth)}

The sensitivity of each approach for the detection of the viruses/viroid in this study was analysed using normalised subsamples (sizes: 1M, 10M, 20M, 30M, 40M and 50M nt). Each of the normalised subsamples from the different sizes was mapped to the consensus sequences of the detected viruses/viroid. The percentages of recovered references (here the consensus sequences) by the viral/viroid nt of each subsample are shown in Fig 3. With the dsRNA approach, the recovered percentages of the genomes reached $100 \%$ at $10 \mathrm{M}$ nt for PEMV2 and PhCMoV, whereas PEMV1 recovered at 20M nt. In cases of PSTVd, the full genome was recovered in some replicates from $20 \mathrm{M}$ to $50 \mathrm{M} \mathrm{nt}$, but the means were lower than $80 \%$. However, for the segmented viruses WHAV2, PNYDV and VCV, the whole genomes could not be recovered even when using $50 \mathrm{M}$ nt. Using ribo-depleted totRNA, the full genomes of PEMV1, PEMV2 and PhCMoV recovered at $10 \mathrm{M}$ nt (Fig 3). The full genome of PSTVd was recovered at $1 \mathrm{M}$ nt. Similar to dsRNA approach, the genomes of WHAV2 and PNYDV were not recovered in all the subsamples. sRNA behaved similar to dsRNA approach with the viruses they both detected. PSTVd recovered at $1 \mathrm{M} \mathrm{nt}$, PEMV2 and PhCMoV at $10 \mathrm{M}$, PEMV1 at 20M, and PNYDV reached about $60 \%$ of the genomes at $50 \mathrm{M}$ nt. Additionally, VCV also reached about $60 \%$ of the genomes at $50 \mathrm{M}$ nt. 


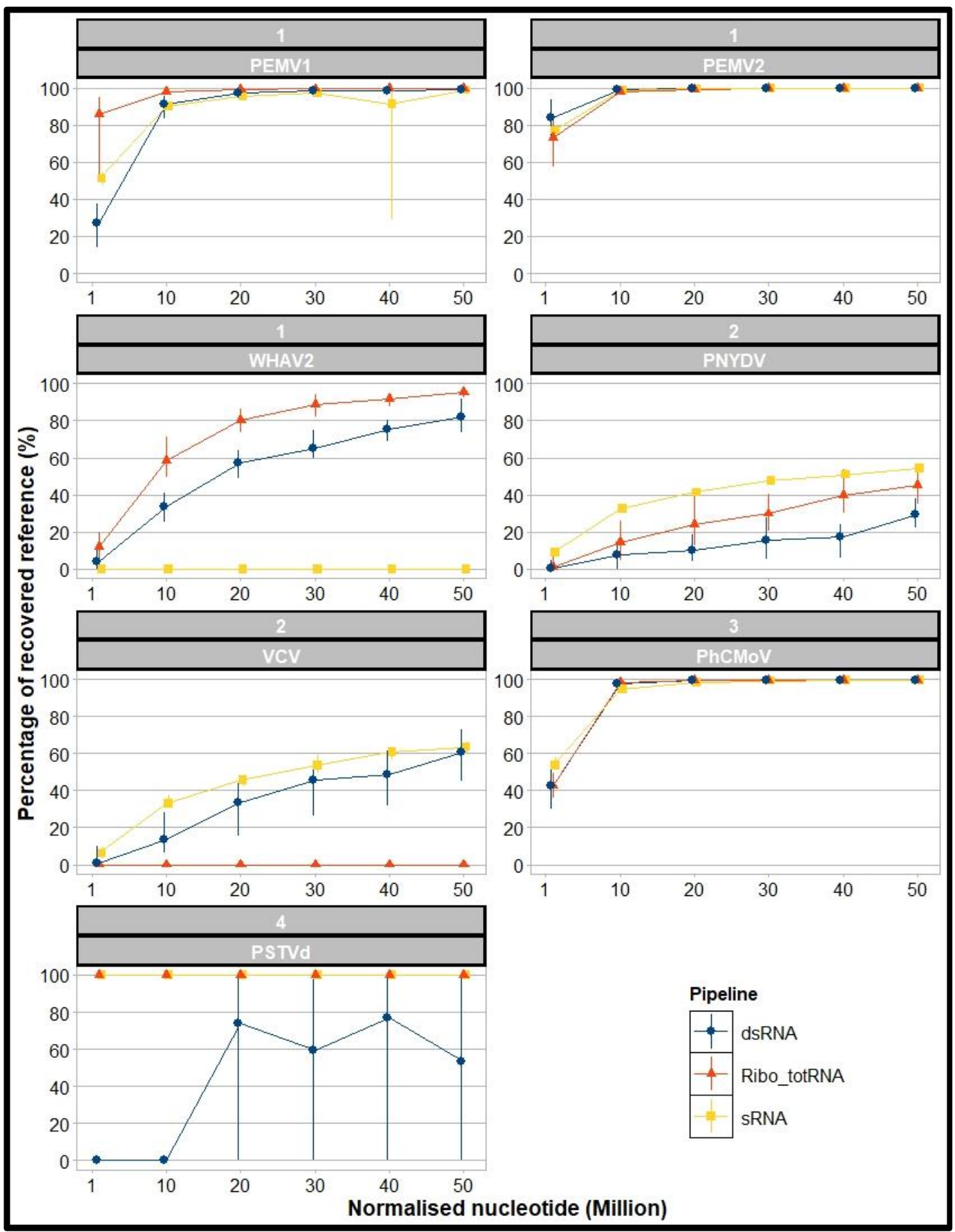

Figure 3: Percentage of reference sequences recovered by the reads of the RNA-based approaches on each of the normalised nucleotide subsamples (sizes: 1M, 10M, 20M, 30M, 40M and 50M nt). The means of each approach are shown as (blue circle: dsRNA, red triangle: ribodepleted totRNA and yellow square: sRNA). The means are joined by lines with same colours. The vertical lines represent the standard deviation of the ten replicates. The strips over each graph are divided into two parts (upper: sample number, lower: virus/viroid acronym).

The dsRNA normalised subsamples had low variation for PEMV1, PEMV2, WHAV2 and PhCMoV, slight variation for PNYDV and VCV, and high variation in case of PSTVd (Fig $3)$. For ribo-depleted totRNA, all the replicates in all the viruses had low variation except 
for PNYDV showed slight variation. sRNA had low variation except in one subsample of PEMV1 i.e., size $40 \mathrm{M}$ nt. The generated contigs by de novo assembly of each normalised subsamples showed the same results (S1 Fig). In Fig 4, the mean depth increased with the size of subsamples in all the three RNA approaches. Regarding the variation, it was the same as the percentages of recovered references of Fig 3.

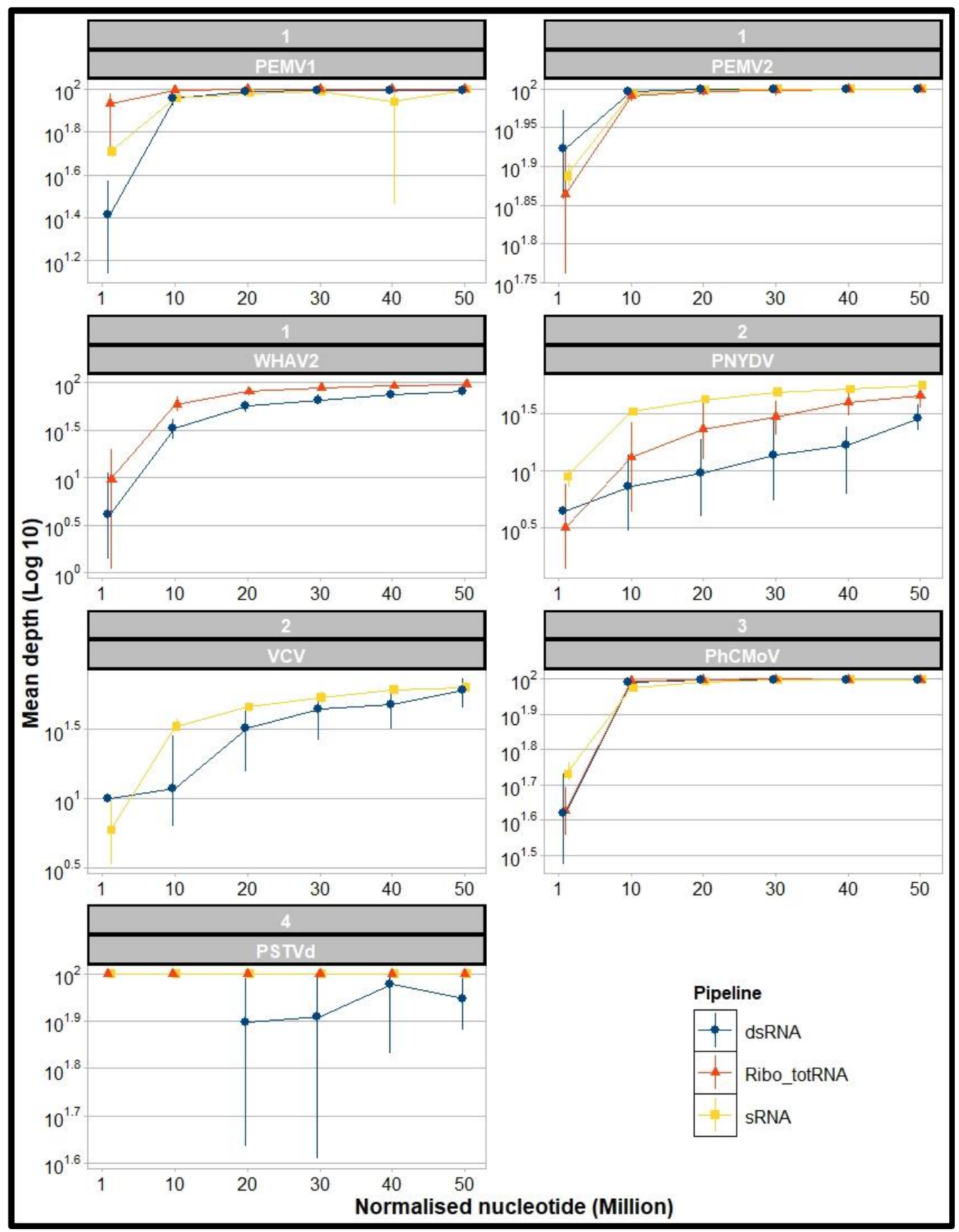

Figure 4: Mean depth of the RNA-based approaches for the detection of the viruses/viroid in this study on each of the normalised nucleotide depth (1M, 10M, 20M, 30M, 40M and 50M nt) for each subsample. The means of each approach are shown as (blue circle: dsRNA, red triangle: ribo-depleted totRNA and yellow square: sRNA). The means are joined by lines with same colours. 
The vertical lines represent the standard deviation of the ten replicates. The strips over each graph are divided into two parts (upper: sample number, lower: virus/viroid acronym).

\subsection{Discussion}

The three viral enrichment approaches (dsRNA, ribo-depleted totRNA and sRNA) used here enabled the detection of the known and unknown plant viruses/viroid in the study. The efficiency of the approaches from extraction to analysis were confirmed by the detection of the spiked viruses (PVEV1 and PVEV2) in all samples by all approaches. dsRNA approach was more efficient than ribo-depleted totRNA and sRNA approaches in terms of virus detection. This is because all the eight viruses and the viroid in this study were detected by the dsRNA approach. While ribo-depleted totRNA and sRNA approaches, each detected seven viruses and the viroid. Three unknown viruses were detected i.e., PEMV2, VCV and WHAV2.

The detection of PEMV2 in mixed infection with PEMV1, is well documented (Doumayrou et al., 2017). Both viruses' genome recoveries were high with the three approaches. VCV is a cryptic (symptomless) virus which occur at very low concentrations in infected tissues of several German varieties of $V$. faba (Blawid et al., 2007). VCV was detected by the dsRNA and sRNA approaches. Furthermore, the ability of HTS to detect cryptic viruses was reported before in (Roossinck, 2011). Interestingly, the four segments of a divergent strain of WHAV2 was identified in sample 1 by dsRNA and ribo-depleted totRNA. WHAV2 was discovered in Hyalopterus pruni and Aulacorthum magnoliae aphids from Wuhan, Hubei province, China in 2013 (Li et al., 2015; Shi et al., 2016b). It has a segmented linear (+ve) ssRNA genome, its virion structure is unknown, and it is not assigned to a virus family yet. However, WHAV2 was phylogenetically grouped in the Jingmenviruses clade (Shi et al., 2016b). Moreover, WHAV2 segments were also detected in three other pea samples collected from Germany and Austria in 2012 and 2013 (data not published). This considered the first detection of WHAV2 sequences in plant tissues and in Europe.

Concerning the recovered number of viral/viroid nt and the mean depth, in general, their amounts were different depends on the species, the sample and the approach. All viral/viroid nt were low in the data of the three approaches, this can be explained by a low virus titre in plant tissues at the time of sampling. As expected, the number of viral/viroid $\mathrm{nt}$ and the mean depth increased with the increase in the size of subsample. Same for the generated contigs, their genome coverage increased with the size of the subsamples.

Linear monopartite ssRNA genomes i.e., PEMV1, PEMV2 and PhCMoV, were detected by all approaches. When the viral nt were mapped to the single stranded linear non-segmented genomes (positive or negative), $10 \mathrm{M}$ to $20 \mathrm{M}$ nt were enough to recover the complete viral genomes. The reasons for the complete recovery at small amount of $\mathrm{nt}$ 
that, dsRNA is generated by RNA viruses as an intermediate in their life cycle, additionally, the removal of ribosomal RNA increases the viral/viroid reads. Regarding viral small RNAs, they are known to be produced during RNA silencing defence of infected plants.

Segmented viruses, regardless of the genomic nature (nucleic acid type, and size and number of the segments), did not recover totally by the three approaches up to $50 \mathrm{M}$ nt. The DNA virus in this study, PNYDV, was not recovered totally by the three RNA approaches, with a slightly higher nt in case of sRNA. As PNYDV has a circular ssDNA multipartite genome, mRNA is synthesised for protein translation. Furthermore, a long dsRNA covering the full-genome is not produced as an intermediate replication by DNA viruses (Wu et al., 2015). As the virus is a phloem restricted virus, a low titre is expected (Vetten et al., 2011). These might be the reasons for the lower recovery of the virus nt. Same conclusion was suggested by (Pecman et al., 2017). VCV was detected by the dsRNA and sRNA approaches. The number of viral nt in the two methods was not significantly different. The low concentration of cryptic viruses such as VCV and its dsRNA nature could be the main reasons that it was not recovered by ribo-depleted totRNA approach. Specially that the denaturation step of dsRNA was $65{ }^{\circ} \mathrm{C}$ for ribo-depleted totRNA approach. WHAV2 might be an aphid virus that is circulating in plant tissues as a vector, this may explain its low titre (Shi et al., 2016b).

For the viroid PSTVd, as it has a short nt sequence (360nt), 1M nt was enough in cases of ribo-depleted totRNA and SRNA, but not for the dsRNA, it required more than $20 \mathrm{M}$ nt. Higher viroid titres in infected tissues may reflect a higher viroid ssRNA and dsRNA (produced during replication) concentrations. Both RNA forms can serve as templates for the Dicer of the RNA silencing (Markarian et al., 2004). These may explain the very high PSTVd nt recovered by the sRNA approach than by dsRNA and ribo-depleted totRNA.

dsRNA extraction consumed less time ( $<1$ hour) in compare to total RNA followed by ribodepletion (about 4 hours) and total RNA followed by sRNA extraction (about 6 hours). Furthermore, the costs of dsRNA extraction per sample was less than the other two approaches (S2 Table). The costs were calculated based on our experience and the prices until early 2019. The main difference between the costs was the cost of the enrichment step.

Additionally, the libraries were sequenced on Illumina platforms MiSeq for both dsRNA and ribo-depleted totRNA, while sRNA on a NextSeq platform. In general, the three approaches generate enough reads to detect the plant viruses and viroid in a given sample. Increasing the number of samples per lane will reduce the costs of platform runs as the generated read, this was also concluded by (Pecman et al., 2017). Moreover, multiplexing by additional barcodes before library preparation can sequence more samples per run and consequently reduce the costs to a more comparable price (Roossinck et al., 2010). 
From these, we recommend sequencing dsRNA for plant virus/viroid detection by HTS, and at least 130 thousand of high-quality reads. The ability of dsRNA enrichment to detect ssRNA (positive and negative), dsRNA and DNA viruses, and viroids was reported before (Rott et al., 2017; Rott et al., 2018). Since most plant viruses produce an intermediate dsRNA or their genome consists of dsRNA and that dsRNA is very stable and can be easily purified, sequencing of dsRNA is therefore a very powerful method for detecting all virus types. There are different dsRNA extraction methods used for HTS. The reasons for using the dsRNA extraction kit mentioned here that it consumes less time $(<1$ hour), uses less milligrams (50 to 200) of plant tissue starting material, does not require PCR amplification in compare to other dsRNA methods extraction methods, in addition to its comparable cost per sample (Kesanakurti et al., 2016; Yanagisawa et al., 2016; Blouin et al., 2016).

Regarding bioinformatic analysis, we recommend after quality control, reads normalisation, followed by de novo assembly of the reads, then BLAST search and mapping to reference based on the BLAST results. The results should be additionally confirmed by RT-PCR. These recommendations can be used as guidelines for viruses/viroids diagnostic.

The study further concludes the ability of HTS to detect known and unknown plant viruses and viroids. This study showed that the performance of the three RNA-based approaches is virus/viroid and sample dependent. We conclude that HTS generated data from the dsRNA approach outcompeted the ones generated from ribo-depleted totRNA and SRNA, and potentially can be used for the detection of all plant viruses and viroids. We also suggest comparing between the different available dsRNA extraction methods to reach the best method.

\subsection{Supplementary}




\begin{tabular}{|c|c|c|c|c|c|}
\hline 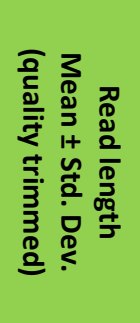 & 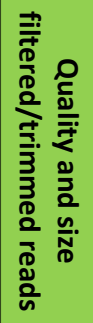 & Iv & ָू & 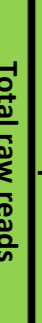 & 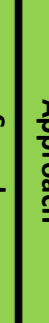 \\
\hline 感 $\underset{\substack{N \\
0}}{\mathbb{N}}$ & 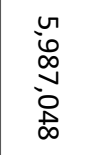 & $\mid \begin{array}{l}\infty \\
0 \\
\dot{\omega} \\
\text { o. } \\
0\end{array}$ & so & $\begin{array}{l}0 \\
0 \\
0 \\
0 \\
0 \\
\delta \\
\sigma\end{array}$ & \multirow{4}{*}{$\begin{array}{l}\text { ñ } \\
\frac{1}{x} \\
\frac{1}{2}\end{array}$} \\
\hline$\underset{v}{S} \underbrace{N}_{1+}$ & $\begin{array}{l}\overrightarrow{5} \\
\stackrel{0}{0} \\
\stackrel{\omega}{\omega} \\
\stackrel{\infty}{\infty}\end{array}$ & 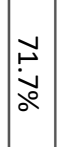 & $\begin{array}{l}\infty \\
\text { in } \\
\text { in }\end{array}$ & 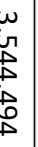 & \\
\hline$\stackrel{\mathscr{g}}{\tilde{w}} \underset{i}{\stackrel{\sim}{w}} \underbrace{\sim}_{1+}$ & 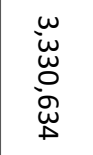 & 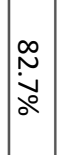 & 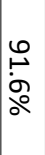 & $\begin{array}{l}\tilde{N} \\
\tilde{N} \\
\tilde{N} \\
\tilde{N} \\
\infty\end{array}$ & \\
\hline 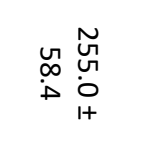 & $\begin{array}{l}\stackrel{N}{\tilde{N}} \\
\tilde{N} \\
\stackrel{\infty}{\infty} \\
\text {. }\end{array}$ & $\begin{array}{l}\infty \\
\stackrel{0}{0} \\
\dot{0} \\
0 \\
0\end{array}$ & $\begin{array}{l}\qquad \\
\stackrel{\leftrightarrow}{*}\end{array}$ & . & \\
\hline$\stackrel{\vec{\oplus}}{\stackrel{\vec{\sigma}}{\vec{\sigma}}}$ & 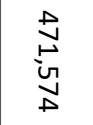 & $\begin{array}{l}\infty \\
0 \\
0 \\
0 \\
0 \\
0 \\
0\end{array}$ & 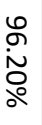 & 吕 & \\
\hline 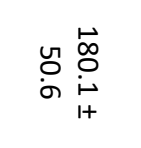 & 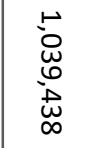 & $\begin{array}{l}\infty \\
0 \\
0 \\
0 \\
0 \\
0\end{array}$ & & 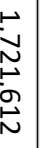 & \\
\hline 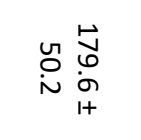 & 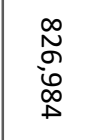 & $\begin{array}{l}\infty \\
0 \\
\tilde{0} \\
0 \\
0\end{array}$ & & 点 & \\
\hline$\stackrel{\leftrightarrow}{\sigma} \underbrace{\stackrel{\leftrightarrow}{\infty}}_{1+}$ & 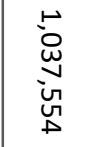 & 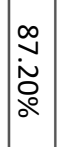 & $\begin{array}{l}\mathscr{c} \\
\dot{w} \\
\dot{w}\end{array}$ & $\begin{array}{c}\stackrel{.}{N} \\
\tilde{w} \\
\tilde{N} \\
\tilde{N}\end{array}$ & \\
\hline $\begin{array}{l}\stackrel{\sim}{+} \\
\infty \\
+ \\
+ \\
\dot{\omega}\end{array}$ & 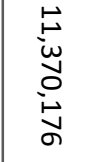 & 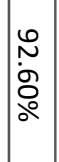 & & $\begin{array}{l}\sigma \\
\sigma \\
o \\
\omega \\
\omega \\
0 \\
\infty \\
\infty\end{array}$ & \multirow[t]{3}{*}{ د } \\
\hline $\begin{array}{l}\tilde{N} \\
\dot{0} \\
1+ \\
+ \\
\dot{D}\end{array}$ & 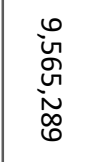 & 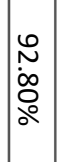 & & 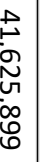 & \\
\hline $\begin{array}{l}\tilde{N} \\
\stackrel{N}{O} \\
1+ \\
\stackrel{+}{D}\end{array}$ & 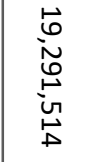 & $\begin{array}{l}0 \\
\stackrel{2}{0} \\
\dot{o} \\
\stackrel{0}{\circ} \\
\end{array}$ & 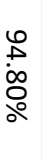 & 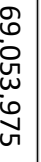 & \\
\hline $\begin{array}{l}\stackrel{\tilde{v}}{\stackrel{H}{*}} \\
\stackrel{+}{+} \\
\dot{\omega}\end{array}$ & $\begin{array}{l}\stackrel{\sim}{\sim} \\
\infty \\
N \\
\infty \\
\stackrel{N}{N}\end{array}$ & 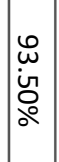 & & 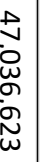 & \\
\hline
\end{tabular}

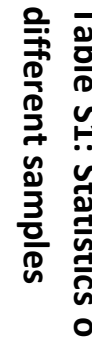

妾

है

वे

오

จ

కั5

疋

음

กั้

๑

立

文

궁

วั้

品

홍

容 


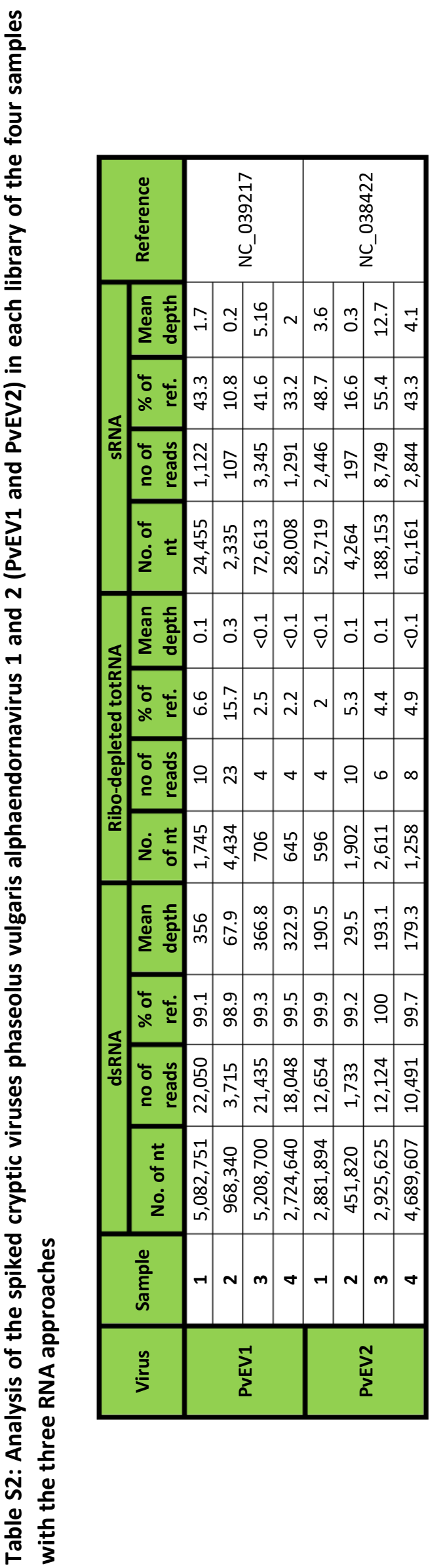


Table S3: Pairwise comparisons of the nucleotide (nt) sequences and the amino acid (aa) sequence identities of Fr HZ11-065 isolates of PEMV1 and PEMV2 proteins with their closest known isolates

\begin{tabular}{|c|c|c|c|c|c|c|}
\hline \multirow{2}{*}{$\begin{array}{c}\text { PEMV } \\
\text { Fr HZ11-065 }\end{array}$} & \multicolumn{2}{|c|}{ Nucleotides } & \multicolumn{4}{|c|}{ Proteins } \\
\hline & Identity & Reference & & ORF & Identity & Reference \\
\hline \multirow{5}{*}{ PEMV1 } & \multirow{5}{*}{$95.7 \%$} & \multirow{5}{*}{ HM439775 } & ORF1 & hypothetical $34 \mathrm{kDa}$ protein & $96.0 \%$ & AD086938 \\
\hline & & & ORF2 & hypothetical protein & $96.1 \%$ & AD086939 \\
\hline & & & ORF3 & RNA-dependent RNA polymerase & $96.4 \%$ & ADO86940 \\
\hline & & & ORF4 & coat protein & $99.5 \%$ & AD086941 \\
\hline & & & ORF5 & aphid transmission protein & $97.6 \%$ & AD086942 \\
\hline \multirow{4}{*}{ PEMV2 } & \multirow{4}{*}{$93.5 \%$} & \multirow{4}{*}{ AY714213 } & ORF1 & hypothetical protein & $92.5 \%$ & ALP43778 \\
\hline & & & ORF2 & RNA-dependent RNA polymerase & $95.6 \%$ & AAU20330 \\
\hline & & & ORF3 & phloem RNA movement protein & $96.5 \%$ & AAU20331 \\
\hline & & & ORF4 & cell-to-cell RNA movement protein & $97.6 \%$ & AAU20332 \\
\hline
\end{tabular}

Table S4: Pairwise comparisons of the four segments' nucleotide sequences and the amino acid (aa) sequences of the predicted proteins of the French isolate of WHAV2 (Fr HZ11-065)

\begin{tabular}{|c|c|c|c|c|c|c|}
\hline $\begin{array}{c}\text { WHAV2 } \\
\text { Fr HZ11-065 }\end{array}$ & \multicolumn{2}{|c|}{ Nucleotides } & \multicolumn{4}{c|}{ Proteins } \\
\hline Segment 1 & $86.4 \%$ & NC_028382 & ORF1 & NS5-like & $96.8 \%$ & YP_009179378 \\
\hline \multirow{2}{*}{ Segment 2 } & \multirow{2}{*}{$90.7 \%$} & \multirow{2}{*}{ NC_028386 } & ORF1 & VP4 & $96.9 \%$ & YP_009179384 \\
\cline { 4 - 7 } & & & ORF2 & VP1 & $90.2 \%$ & YP_009179385 \\
\hline \multirow{2}{*}{ Segment 3 } & $85.2 \%$ & \multirow{2}{*}{ NC_028383 } & ORF1 & NS3-like & $95.5 \%$ & YP_009179379 \\
\hline \multirow{2}{*}{ Segment 4 } & \multirow{2}{*}{$85.6 \%$} & \multirow{2}{*}{ NC_028387 } & ORF1 & VP2 & $92.0 \%$ & YP_009179386 \\
\cline { 4 - 7 } & & & ORF2 & VP3 & $96.6 \%$ & YP_009179387 \\
\hline
\end{tabular}




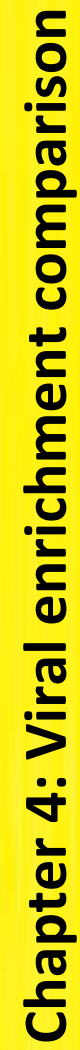

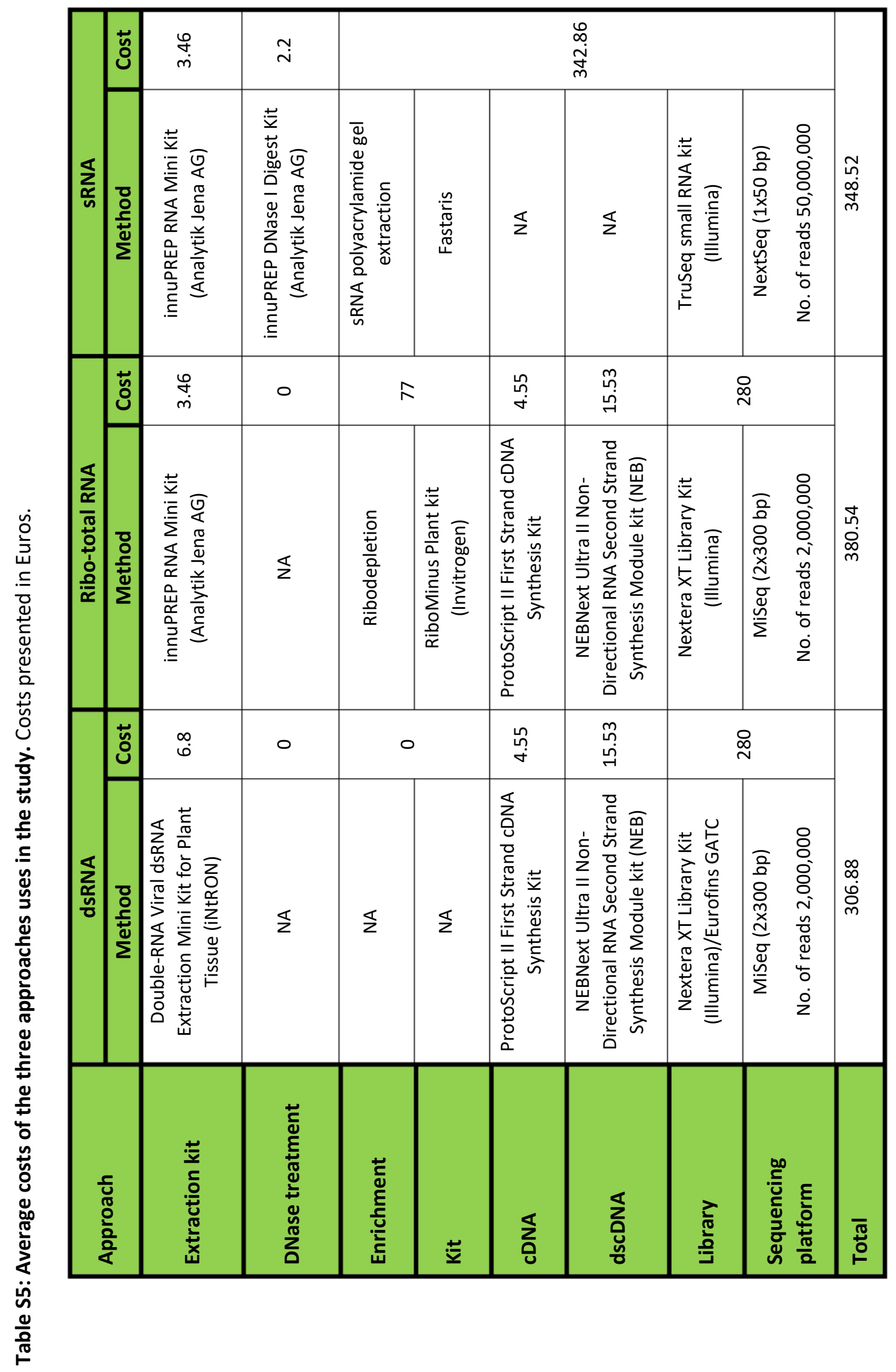




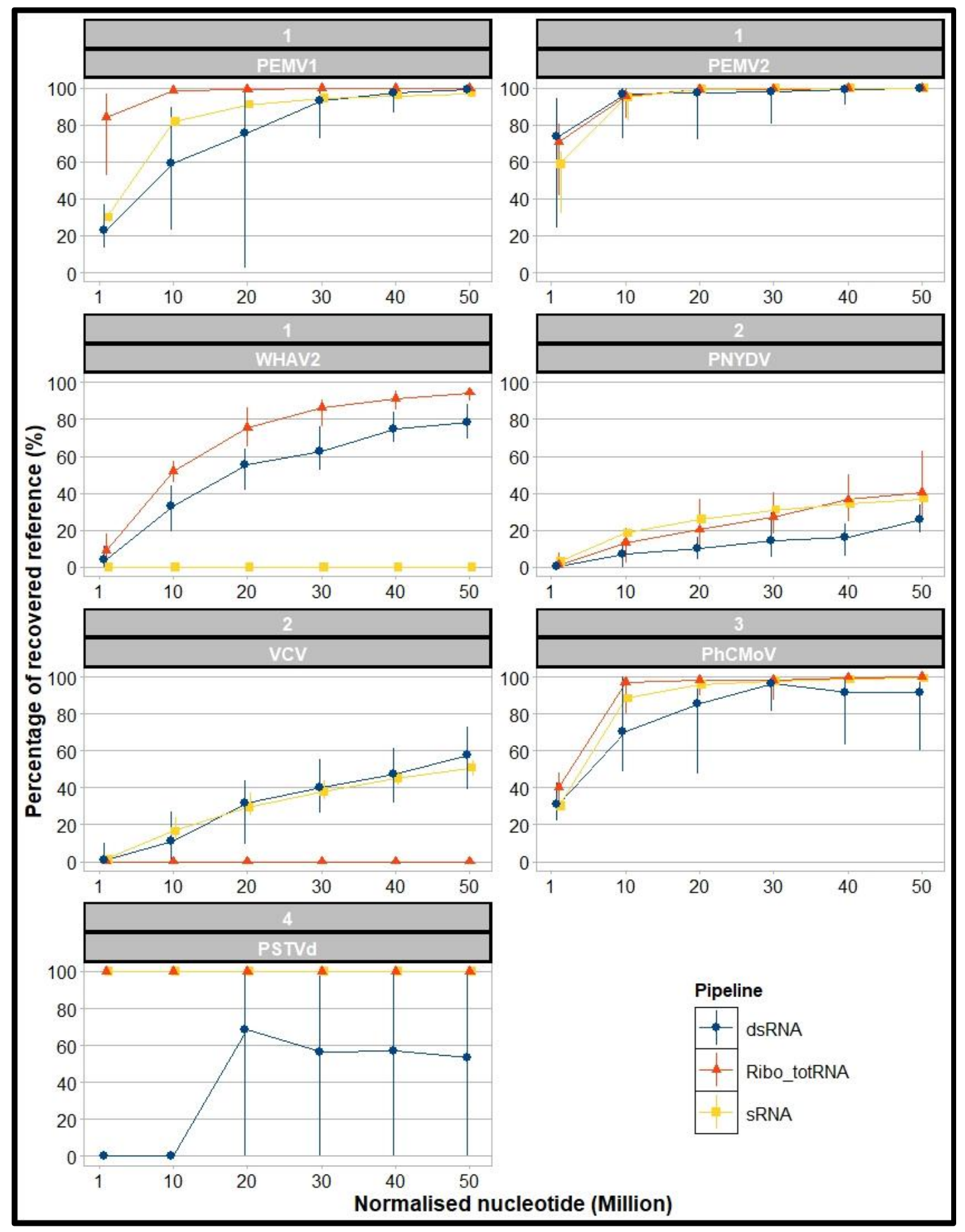

Figure S1: Percentage of reference sequences recovered by the produced contigs of the RNAbased approaches on each of the normalised nucleotide subsamples (sizes: 1M, 10M, 20M, 30M, 40M and 50M nt). The means of each approach are shown as (blue circle: dsRNA, red triangle: ribo-depleted totRNA and yellow square: sRNA). The means are joined by lines with same colours. The vertical lines represent the standard deviation of the ten replicates. The strips over each graph are divided into two parts (upper: sample number, lower: virus/viroid acronym). 
$160 \mid \mathrm{P}$ a g e 


\section{Part two: Virus-vector-host interactions}


$162 \mid \mathrm{P}$ a g e 


\section{Chapter 5: Aphid transmission of nanoviruses: a review}

Yahya Zakaria Abdou Gaafar and H. Ziebell 


\subsection{Abstract}

The genus Nanovirus is composed of plant viruses that predominantly infect legumes and can cause devastating crop losses. Nanoviruses are vectored by various aphid species. The transmission occurs in a circulative, non-propagative manner. It was long suspected that a virus-encoded helper factor would be needed for successful transmission by aphids. Recently, this helper factor was identified as the nanovirus-encoded nuclear shuttle protein (NSP). The mode of action of NSP is currently unknown - in contrast to other helper factors that e.g., facilitate binding of virus particles to receptors within the aphids' stylets. In this review, we are summarizing the current knowledge about nanovirus-aphid vector interactions.

\subsection{Introduction}

Viruses are one of the main constraints for successful and sustainable crop production. Viral infections can reduce both yield and quality of the produce but may also cause total crop losses. Plant viruses can be transmitted from plant to plant via different routes of transmission; they can be transmitted mechanically (i.e., handling of plants or harvesting of fruits, root contacts, nutrient solutions within closed cropping systems etc.), by seeds, pollen, or vectors such as fungi, mites, nematodes, aphids and other insects. Phloem-feeding insects such as aphids (Hemiptera, Aphididae) are the most common vectors of plant viruses (Hogenhout et al., 2008). Aphids can be found worldwide, and many aphid species are able to act as virus vectors for one or more virus species (Stevens \& Lacomme, 2017). Half of the insect-vectored known plant viruses depend on aphids for their transmission (Nault, 1997).

Traditionally, four different mechanisms are used to describe virus transmission by aphids (Hogenhout et al., 2008): non-persistent transmission (NP), semi-persistent (SP) transmission, persistent circulative (non-propagative) transmission (PC) and persistent propagative (PP) transmission. Viruses that are transmitted NP are retained in the stylets and can only be transmitted for a few minutes after acquisition; the ability to transmit the viruses are lost within a few minutes and upon insect molting. Most SP viruses are retained in the foregut while some are retained in the stylets. and transmission can occur minutes to hours to a few days after acquisition; again, viruses are lost upon molting. However, some SP transmitted viruses are retained in stylets (Uzest et al., 2007). No latent period (the time between acquisition access period [AAP] and inoculation access period [IAP]) is required for the transmission of NP or SP viruses contrary to persistentlytransmitted viruses (Nault, 1997; Hogenhout et al., 2008). Persistently transmitted viruses are classified as either circulative (and mainly non-propagative) viruses and propagative viruses. Upon acquisition, these viruses circulate through the insect vectors, moving from the gut lumen into the hemolymph and from there into the salivary glands from which the virus particles can be inoculated into new plants upon feeding. In addition, while non- 
propagative viruses are assumed not to replicate within their insect vectors, viruses transmitted in a persistently propagative manner do.

Most knowledge about virus translocation pathways of PC transmitted viruses within aphids comes from research on members of the Luteoviridae family (Garret et al., 1993; Gray \& Gildow, 2003). It is suspected that luteovirids enter the gut epithelium by endocytosis before being released into the hemocoel by (Figure 1) (Garret et al., 1993; Gray \& Gildow, 2003). For some luteovirids, such as beet western yellows virus, a minor read-through protein (RTP) has been shown to be involved in aphid transmission together with the coat protein (CP); determining whether the virions can accumulate in the midguts or in both midguts and hindguts of aphids (Brault et al., 1995; Brault et al., 2000; Brault et al., 2005). 


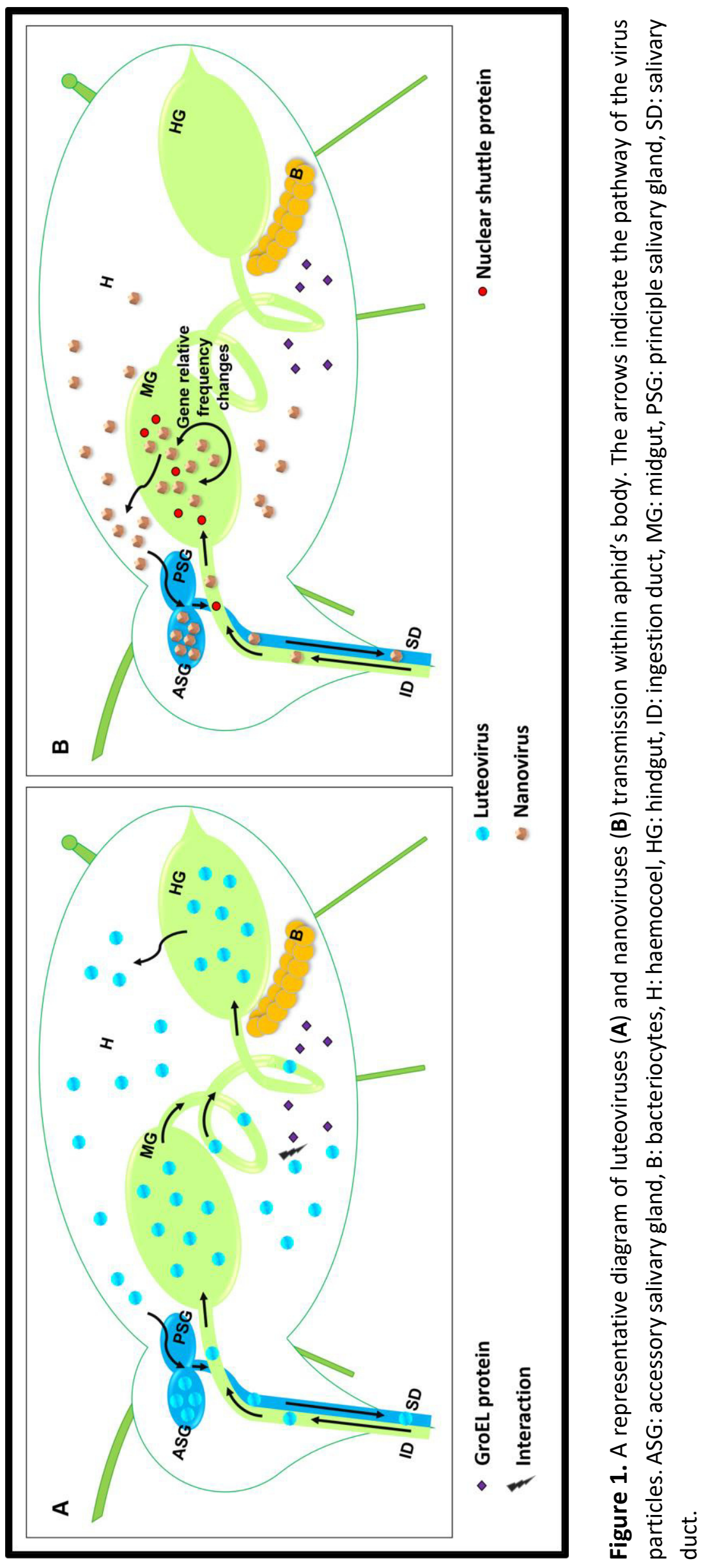


The virions are then acquired through into the hemocoel, regardless whether the aphid was a vector of particular species (Gildow, 1993; Gildow et al., 2000). The virus particles are transported in vesicles through the cytoplasm of epithelial cells. Then the vesicles fuse with the basal plasmalemma and release particles into the interspace between the membrane and the basal lamina (Gray et al., 2014). The virions move across the basal lamina into the hemocoel. Within four hours of the viroins' arrival in the alimentary canal lumen, they could be observed in the gut epithelial cells and within 8 hours they were detected in the hemolymph (Garret et al., 1996). Luteovirids then move across the accessory salivary gland (ASG) cells in a pathway similar to that used to cross the gut (Gray \& Gildow, 2003). In non-vector aphids, luteovirids are unable to penetrate the ASG suggesting that the basal lamina and the basal plasmalemma act as barriers to transmission (Gray et al., 2014). With longer feeding periods, the number of epithelial cells containing the virions increased (Garret et al., 1996). Mutational analysis of the CP and the RTP of turnip yellows virus (TUYV) and potato leafroll virus (PLRV), both poleroviruses, identified that they affected both aphid transmission and/or plant-virusinteractions (Bruyère et al., 1997; Brault et al., 2000; Lee et al., 2005; Kaplan et al., 2007; Peter et al., 2008). CP was sufficient to transcytose virions of the gut to the hemocoel while it was suggested that the RTP facilitate the docking of the virions to the epithelial cells (Brault et al., 2005; Chavez et al., 2012). Moreover, the CP-RTP appeared to be required for interacting and passing through the ASG membranes (Bruyère et al., 1997; Brault et al., 2000; Peter et al., 2008). Furthermore, Cilia and colleagues suggested critical virion-host protein interactions required for aphid transmission of cereal yellow dwarf virus (CYDV), or that the virus infection modulates phloem protein expression to favor aphid virus uptake (Cilia et al., 2012). Investigating the particles of luteoviruses, which are transmitted in a circulative non-propagative manner, showed that they are transported across cells through membrane vesicles, preventing any contact between the virus and the cytoplasm of aphid's cells (Brault et al., 2007). Yet there is no evidence that nanoviruses have the same manner.

\subsection{Nanoviruses}

The genus Nanovirus (family: Nanoviridae) currently comprises eight species accepted by the International Committee on the Taxonomy of Viruses (ICTV). Nanoviruses predominantly infect legumes which are important crops for human and animal consumption and are also used to improve soil health or as green manures (Johnstone \& Mclean, 1987; Vetten et al., 2011; Foyer et al., 2016; Gaafar et al., 2016). Two potentially new nanovirus species have been recently discovered from Sophora alopecuroides L. (also a legume) and Petroselinum crispum (Mill.) Fuss (belonging to the family of Apicacea) (Heydarnejad et al., 2017; Vetten et al., 2019). Nanovirus infection can cause a variety of different symptoms e.g., severe yellowing and dwarfing of plants or necrosis of plant tissues (Vetten et al., 2011; Saucke et al., 2019). In some cases, the infection can lead to early death of the host plants thus leading to crop failures (Grylls \& Butler, 1956; Makkouk 
et al., 1994; Makkouk et al., 1998; Kumari \& Makkouk, 2007; Saucke et al., 2019). Nanoviruses have been found in Australia, Asia, Northern Africa and the Middle East (Grylls \& Butler, 1956; Chu \& Helms, 1988; Makkouk et al., 1994; Sano et al., 1998; Abraham et al., 2012); a sporadic outbreak of faba bean necrotic yellows virus (FBNYV) has been reported in Spain (Ortiz et al., 2006). In 2009, the nanovirus pea necrotic yellow dwarf virus was discovered for the first time in Central Europe (Germany) (Grigoras et al., 2010a); in subsequent surveys this and other nanovirus species were discovered in Europe (Grigoras et al., 2014; Gaafar et al., 2016; Gaafar et al., 2017; Gaafar et al., 2018a; Vetten et al., 2019).

Nanoviruses possess a multipartite single-stranded circular DNA genome of at least eight components (Vetten et al., 2011). Each component is about $1 \mathrm{~kb}$ nucleotides in size and encodes a single protein. Each DNA component is encapsidated separately in a single non-enveloped icosahedral virion ( $T=1$ symmetry) (Vetten et al., 2011). Several nanovirus-associated alphasatellite DNAs occur frequently with natural infection of nanoviruses; however, their biological function is unclear (do they impact infectivity and symptomology? Do they influence the host range or aphid transmissibility?) (Gaafar et al., 2018a; Gallet et al., 2018; Heydarnejad et al., 2017). Nanoviruses can evolve rapidly by mutations, recombination and reassortment (Grigoras et al., 2010b; Grigoras et al., 2014) and are closely related to babuviruses, the second accepted genus in the Nanoviridae family (Vetten et al., 2011). Only six genomic components are known for babuviruses, which infect banana and cardamom.

\subsection{Aphid transmission of nanoviruses}

Nanoviruses are restricted to the phloem of infected host plants and are therefore not transmissible by seeds or mechanical means (Vetten et al., 2011). They are dependent on vectors for the transmission from plant to plant. In nature, they are transmitted by a range of aphid species, e.g., the pea aphid Acyrthosiphon pisum Harris, the cowpea aphid Aphis craccivora C.L. Koch or the bean aphid A. fabae Scopoli. It is unclear whether nanoviruses can be transmitted by other aphid species such as Aphis gossypii Glover or Macrosiphum euphorbiae Thomas (Vetten et al., 2016). Nanoviruses are transmitted in a PC manner (Franz et al., 1998; Vetten et al., 2011). This means that aphids can only ingest the virus particles from infected plants when they are actively feeding on phloem sap. The viral particles need to cross the cellular barriers from the aphids' gut epithelium into the hemolymph; from the hemolymph, translocation to the salivary glands needs to occur (Figure 1) (Blanc et al., 2014). The viruliferous aphid can then inject the particles with saliva during feeding on new plants.

For successful transmission of wild-type virus to new host plants, all eight genomic components need to be acquired, translocated within the vector and transmitted to new hosts. Interesting, Sicard and colleagues discovered that the relative frequencies of certain components of faba bean necrotic stunt virus (FBNSV) changed reproducibly, not 
only depending on the host plant but also within different aphid vectors (Sicard et al., 2013; Sicard et al., 2015). It appears that these changes occur in early stages of the virus cycle in aphids but there were no further changes in the segment relative frequencies thereafter when the virions are translocated into the salivary glands (Sicard et al., 2015). These frequencies were affected partially by the initial genomic formula from the source plant. These effects were observed in three FBNSV vector species A. pisum, A. craccivora and Myzus persicae Sulzer. Interestingly, similar amounts of FBNSV gene copy numbers (GCN) accumulated and changes in the FBNSV genomic formula were also observed in the non-vector species A. gossypii (Sicard et al., 2015).

The most detailed transmission studies have been carried out with FBNYV. FBNYV infects several food and fodder legumes in west Asia and North Africa and Europe, and has caused huge epidemics in the past (Makkouk et al., 1994; Kumari et al., 2009; Ortiz et al., 2006). Franz and colleagues investigated the minimum AAP of FBNYV for two vector species, A. craccivora and A. pisum. They found that the AAPs were similar for both species, ranging from 15 to 30 minutes. Additionally, the minimum IAP was determined for both aphid species and ranged between 5 and 15 minutes. However, longer AAPs and IAPs resulted in higher virus transmission rates. After 72 hours of feeding on infected plants, many aphids retained and transmitted FBNYV throughout their life in an erratic manner (Franz et al., 1998). FBNYV particles were not lost during molts, i.e., the aphids remained viruliferous for up to one month, but FBNYV was not passed on to parthenogenetic offspring and no indication for viral replication within the aphids was found. Interestingly, nymphs were more efficient in transmitting FBNYV than adult aphids, a phenomenon also observed in other persistently transmitted viruses (Simons, 1953; Zhou \& Rochow, 1984; Damsteegt \& Hewings, 1986; van den J. M. Heuvel, 1991).

In further studies, Franz and colleagues have shown that no transmission occurred when using purified virions from artificial diets or directly microinjected into the vector's hemocoel would lead to successful transmission of FBNYV (Franz et al., 1999) It was, however, possible to infect plants directly with purified virions using gold particle bombardment (Franz et al., 1999). These bombarded plants acted as reservoir of FBNYV for further successful virus acquisition by aphid and FBNYV transmission to uninfected target plants (Franz et al., 1999). Franz and colleagues (1999) suggested that a helper factor (HF) was required for successful aphid transmission and that the HF was either absent or non-functional in purified virus solution. Their hypothesis was supported by complementation experiments using two different FBNYV strains: when feeding on plants infected with one strain, acquisition and transmission of the second strain from artificial diets was possible (Franz et al., 1999). Similarly, microinjection of a second, purified, strain into aphids that had fed on plants infected with the first strain led to successful transmission. The authors speculated that the HF would be an intermediate viral protein, attaching the virus capsid protein $(\mathrm{CP})$ to receptors in the vector to facilitate virus 
transport across the hemolymph - salivary gland interface (Franz et al., 1999). However, at the time they were unable to identify the nature of the HF.

The availability of infectious clones for nanoviruses including FBNYV and FBNSV allowed to study the functions of the genomic components of nanoviruses in more details (Timchenko et al., 2006; Grigoras et al., 2009; Grigoras et al., 2018). Using agroinoculation of all eight components of FBNSV, plants showed severe symptoms 10 to 14 days post inoculation (Grigoras et al., 2018). Omitting one component at a time, no change in infectivity or symptomology was found for DNA-C (encoding a cell-cycle-link protein [Clink]) or DNA-U4 (encoding for a protein of unknown function). Omission of DNA-R (encoding the master replicase protein [M-Rep]), DNA-S (coat protein) or DNA-M (movement protein [MP]) resulted in the absence of infection on inoculated plants, therefore no symptoms could be observed. Omission of DNA-U2 or DNA-U1 (both encoding hypothetical proteins of unknown function) lead to reduced symptom severity, but virus transmission from inoculated plants by cowpea aphids was still possible. More strikingly, by omitting DNA-N (encoding for the nuclear shuttle protein (NSP)), plants became infected showing similar symptoms as when all eight viral components were inoculated, whereas the aphid transmission was completely abolished (Grigoras et al., 2018). The virions that were produced within the plants inoculated with the seven components excluding DNA-N, accumulated to similar titers and were virtually morphologically and structurally indistinguishable from wild-type FBNSV particles. Introduction of a 13 amino acid tag at the carboxy-terminus of NSP also abolished aphid transmission. Interestingly, when the seven FBNSV components minus NSP were combined with DNA-N from a different nanovirus, pea necrotic yellow dwarf virus (PNYDV), the aphid transmission was restored indicating that this protein is the helper factor that has been previously proposed by Franz and colleagues (1999).

\subsection{What do we know about NSP?}

DNA-N of both nano- and babuviruses encodes the NSP. NSP of the babuvirus banana bunchy top virus (BBTV) was preferentially targeted to the nucleus of infected cells when expressed alone, but in the presence of the viral movement protein, NSP was relocalized to the cell periphery (Wanitchakorn et al., 2000). Its function has been mainly inferred from comparisons with the homologue proteins of the closely related geminiviruses (Wanitchakorn et al., 2000; Krapp et al., 2017). Supposedly, NSP shuttles replicated viral DNA out of the nucleus of infected cell. NSPs of geminiviruses i.e., cabbage leaf curl virus (CaLCuV), tomato golden mosaic virus (TGMV) and tomato crinkle leaf yellows virus (TCrLYV) were found to interact with NSP-interacting kinases (NIKs) in vitro (Fontes et al., 2004; Mariano et al., 2004). NIKs are leucine-rich-repeat (LRR) receptorlike-kinases (RLKs) and are membrane localized protein. LRR-RLKs are involved in plant developmental processes and/or resistance response (Gómez-Gómez \& Boller, 2000; Jinn et al., 2000; Fontes et al., 2004). Fontes and colleagues found a positive correlation between infection rate and loss of NIK function (Fontes et al., 2004). In vitro binding 
between the NSPs and NIKs inhibit NIK kinase activity and prevent the signal transduction pathway activation that would trigger an antiviral defense response (Fontes et al., 2004; Carvalho et al., 2008; Santos et al., 2009; Santos et al., 2010).

In infected faba beans, FBNSV-NSP could only be localized in the phloem-tissue where also FBNSV-CP was detected (Grigoras et al., 2018). Using green fluorescence protein tagging and bimolecular fluorescence complementation (BiFC), Krenz and colleagues were able to localize NSP of PNYDV in the nucleus and in the cytoplasm of infected plant cells (Krenz et al., 2017). Using BiFC, PNYDV-NSP was found to interact with the stress granule component G3BP which led to a speculation of the involvement of NSP in modulation of the plant stress response pathway (Krapp et al., 2017). Additionally, NSP was found to interact with the M-Rep, encoded by DNA-R, which may affect the virus infection cycle (Krapp et al., 2017). Krenz and colleagues hypothesized that NSP may regulate the virus replication by interacting with $M-$ Rep as in the case of the geminivirus REn (Hanley-Bowdoin et al., 2013; Krapp et al., 2017). Furthermore, the NSP was found to self-interact in BiFC experiment in a yeast two-hybrid assay (Krenz et al., 2017).

A recent study on the closely related BBTV found that NSP was located in both the nucleus and the cytoplasm of infected Nicotiana benthamiana Domin (Ji et al., 2019). The presence of BBTV-NSP affected the cellular distribution of BBTV-CP in colocalization experiments in planta. Co-immunoprecipitation verified the interaction between the BBTV-NSP and BBTV-CP suggesting that BBTV-NSP relocates BBTV-CP in infected cells (Ji et al., 2019). The direct role of NSP in nanovirus acquisition, translocation within aphids and/or inoculation of uninfected plants is currently unknown. It is also unclear how NSP does interact with nanovirus virions or other viral proteins in planta, as these interactions were only shown in model plants.

\subsection{Virus translocation within aphids}

Using immunofluorescence, Watanabe and colleagues showed that BBTV coat protein (CP), encoded by DNA-S, localizes in the anterior midgut cells of Pentalonia nigronervosa Coquerel aphids (Bressan \& Watanabe, 2011). The labelling intensity suggested high concentration accumulation in epithelial cells, but no accumulation could be observed in the posterior midgut or hindgut of aphids. In addition, accumulation was observed in principal salivary glands but not in the accessory salivary glands (Bressan \& Watanabe, 2011) In further studies, Bressan and Watanabe used PCR and immunofluorescent assays to examine possible translocation pathways over time (Watanabe \& Bressan, 2013). They observed a progressive internalization of BBTV from the gut lumen to the anterior midgut, where accumulation occurred, followed by translocation into the principal salivary glands via the hemolymph (Watanabe \& Bressan, 2013). However, they suggested also an alternative route whereby direct movement of BBTV from the anterior midgut to the principal salivary gland would be possible. 
To address these possibilities, they used co-labelling assays of BBTV and cellular compartments of the aphid vectors (Watanabe et al., 2016). These experiments suggest that an endosome-independent process is used by BBTV for internalization through the gut tissue. In contrast to the cellular translocation mechanisms used by luteovirids, BBTV appears to use endocytosis-independent processes for internalization that does not include endosomes, clathrin- and caveolae-mediated endocytosis, phagocytic uptake or raft-mediated cytosis (Watanabe et al., 2016). In transmission electron microscopy studies, large numbers of vesicles were observed in the anterior midgut of BBTV-carrying aphids but not in aphids that were reared on healthy banana plants (Vetten et al., 2016)However, it needed to be confirmed that these vesicles contain BBTV particles.

Circulative plant viruses such as luteovirids and geminiviruses were found to bind to GroEL proteins, produced by endosymbiotic bacteria (Buchnera aphidicola) inhabiting their vectors (Munson et al., 1991; Kliot \& Ghanim, 2013). Such interaction seems to protect the virus particles from degradation in the aphids' haemocoel. To date, there is no evidence that nanovirids interact with the GroEL proteins. Although GroEL proteins from Buchnera were detected in the hemolymph of $P$. nigronervosa, no interactions with BBTV virions could be observed using immunocapture PCR, dot blot and far-western blot analyses (Watanabe et al., 2013). Nanovirids translocate in large clusters of virions which may protect individual virions from degradation and could prevent them from interacting with proteins such as GroEL (Vetten et al., 2011; Watanabe et al., 2013; Vetten et al., 2016). It is also possible that NSP assists in preventing degradation in the hemocoel. To date, the interactions of BBTV-NSP and the translocation of virions through the aphid vector have not been investigated.

\subsection{Concluding remarks}

Even though we have seen a huge advancement in nanovirus research in the recent years, many questions regarding the interactions of nanoviruses with their hosts and vectors remain. As more and more nanoviruses and nanovirus-associated satellites are being discovered, it is necessary to understand these interactions in order to prevent nanovirus epidemics as we have seen in the past. As with all plant virus diseases, no curative methods are available once a plant is infected; preventive measures such as planting virus-resistant varieties (if available) or preventing spread by vectors by decreasing vector population for example. It appears that the mode of nanovirus transmission by aphids is more complicated than previously thought. It is noteworthy that NSP has been described as helper factor necessary for successful transmission by aphids. However, its role and mode of action during the transmission process currently remains a mystery. Additionally, it needs to be confirmed whether on the self-interaction between NSPs found in in vitro studies play a role within the aphids or within the transmission process. We also do not know if other viral proteins such as the products of DNA-U1, -U2 and -U4 or the associated alphasatellites influence aphid transmission or virus-vector interactions. It was shown that M-Rep interacts with both the CP and NSP and may 
therefore also influence virus-aphid interactions. It is even more important to identify the motifs which interact with the aphid and host cells for trafficking.

It appears that the mode of nanoviruses aphid transmission not as trivial than it was suggested before. Although both CP and NSP are required for successful transmission, the observation that the GCN of the different segments are host-dependent and can change in aphid vectors raises the question whether these "virus formulas" are a type of host/vector adaptation and whether these changes are necessary for virus transmission or not. Additional studies on the relation of these changes and the erratic retention and transmission of nanoviruses by aphids are clearly required.

Furthermore, studying aphid probing and feeding activities on nanovirus-infected plants (before, during, or after virus acquisition) is crucial. In addition, we need data on aphid fitness on nanovirus-infected plants as it is currently not known if and how nanovirus infection influences its vectors, something which has been shown for other viruses (Alvarez et al., 2007; Ziebell et al., 2011). There are many more research questions that need to be addressed in this exciting topic of nanovirus-vector-host plant interactions. 
Chapter 6: Probing and feeding behaviours of Acyrthosiphon pisum change on nanoviruses-infected faba beans

Yahya Zakaria Abdou Gaafar, Stefan Vidal and H. Ziebell 


\subsection{Abstract}

The probing and feeding behaviours of the pea aphid, Acyrthosiphon pisum (Hemiptera: Aphididae) on nanoviruses- infected faba bean (Vicia faba) were investigated using electrical penetration graph (EPG). We assessed the behaviours of $A$. pisum on $V$. faba each infected with a different nanovirus i.e., FBNYV and PNYDV. Moreover, we studied the effect of DNA-N, the aphid transmission helper component, on the aphid probing and feeding behaviours. The results showed that nanovirus infection changes the behaviours of $A$. pisum, by making the plants less attractive to the aphids. Moreover, the absence of DNA-N did not affect the behaviour of the aphids.

\subsection{Introduction}

Viruses of the genus Nanovirus (family: Nanoviridae) are multipartite plant DNA viruses that infect predominantly legumes and can cause crop losses (Vetten et al., 2011; Makkouk et al., 2014). Nanoviruses have been reported from Asia, Australia, Europe, and Africa (Gutierrez et al., 1971; Makkouk et al., 1994; Ortiz et al., 2006; Kumari et al., 2009). The genus Nanovirus has eight assigned species recognized by the International Committee on Taxonomy of Viruses i.e., Black medic leaf roll virus (BMLRV), Faba bean necrotic stunt virus (FBNSV), Faba bean necrotic yellows virus (FBNYV), Faba bean yellow leaf virus (FBYLV), Milk vetch dwarf virus (MVDV), Pea necrotic yellow dwarf virus (PNYDV), Pea yellow stunt virus (PYSV) and Subterranean clover stunt virus (SCSV). Their genomes consist of eight circular ssDNA components (DNA-C, $-M,-N,-R,-S,-U 1,-U 2$ and -U4) of about $1 \mathrm{~kb}$ each (Vetten et al., 2011). Each component is encapsidated separately in nonenveloped virions (Vetten et al., 2011). Additionally, several associated alphasatellite DNAs can also be detected (Gaafar et al., 2018a; Gallet et al., 2018).

Nanoviruses are phloem-restricted and are transmitted by aphids e.g., Aphis craccivora and Acyrthosiphon pisum (Franz et al., 1998; Vetten et al., 2016). Nanovirus aphid transmission occurs in a circulative non-propagative manner, though a recent study showed a more complex manner (Sicard et al., 2015). When the aphids feed on infected plants, the viruses are acquired with the phloem sap, then translocate from the gut to the haemolymph to the salivary glands in unknown mechanism without replicating or expressing their genes. Sicard and colleagues found reproducible changes in the relative frequencies of some of the FBNSV components in A. pisum, A. craccivora, and Myzus persicae aphids compared to the host plants they fed on (Sicard et al., 2015). These changes occurred inside the gut but did not change later when the virus particles are in the salivary glands (Sicard et al., 2015). Thus, they suggested that the canonical circulative non-propagative transmission might not fit with nanoviruses.

Franz and colleagues suggested that a virus helper factor (HF) is required for FBNYV aphid transmission (Franz et al., 1999). They also suggested that the HF might attach the nanovirus coat protein to receptors in the aphids (Franz et al., 1999). Grigoras 
and colleagues found that the nuclear shuttle protein (NSP) encoded by DNA-N is the required HF for PNYDV aphid transmission (Grigoras et al., 2018). However, it is currently unclear at which step of the transmission pathway NSP is involved.

Several factors can influence the virus-aphid-plant interactions, including the chemical composition and physical structure of the plant (Guo et al., 2014). It has also been shown that virus infection can affect the feeding behaviour of their vectors and therefore the transmission (Ziebell et al., 2011). Electrical penetration graph (EPG) is a powerful tool for investigating feeding behaviour of aphids (Tjallingii, 1978, 1985). It has been used to demonstrate that the feeding behaviour of non-viruliferous Myzus persicae was enhanced on potato leafroll virus (PLRV) infected potato plants (Alvarez et al., 2007).

With the recent advancement of nanovirus research, we were interested whether nanovirus infection would alter the feeding behaviour of non-viruliferious aphids. We therefore aimed to study the effects of nanoviruses infection on aphid probing and feeding behaviours using the EPG technique, comparing the effects of two nanoviruses (FBNYV and PNYDV) on the feeding and probing behaviour of $A$. pisum on faba beans (Vicia $f a b a)$. Moreover, to assess the effect of DNA-N absence on these behaviours.

\subsection{Materials and methods}

\subsubsection{Plants and aphids:}

V. faba plants (variety: Tattoo) were grown in a greenhouse at $16 / 8 \mathrm{~h}$ light/dark (natural daylight with additional growth light Phillips IP65, $400 \mathrm{~W}$ ) and $22^{\circ} \mathrm{C}$. In all experiments, 11 days old seedlings were used.

The pea aphids, A. pisum (Harris) (JKI clone), used in this study were reared continuously in an insect-proof cage on faba bean plants in a climate chamber at $22^{\circ} \mathrm{C}$ and $16 / 8$ light/dark (sodium high pressure lamps). Fresh plants were added frequently to the cage to prevent overcrowding.

To synchronize the aphids for the experiments, 10 viviparous adults were placed on V. faba plants in a cage in greenhouse chamber at $22^{\circ} \mathrm{C} 16 / 8 \mathrm{~h}$ light/dark. After 24 hours, the adults were removed, and the newly born nymphs were left for about 13 days. This was repeated daily to have enough aphids of almost the same age (about $24 \mathrm{~h}$ difference) for each day of recording.

\subsubsection{Endosymbiont detection and confirmation:}

DNA was extracted from three sets of 10 aphids collected in a $2 \mathrm{ml}$ tube according to (Shahjahan et al., 1995). PCR was performed using primers for $A$. pisum endosymbionts (Tsuchida et al., 2002). 


\subsubsection{Virus isolates maintenance and confirmation:}

Three nanoviruses were used in this study i.e., faba bean necrotic stunt virus (FBNSV) isolate JKI-2000 from Ethiopia, faba bean necrotic yellows virus (FBNYV) isolate AZ originally from Azerbaijan (Grigoras et al., 2014), and pea necrotic yellow dwarf virus (PNYDV) isolate Drohndorf-15 originally from Germany (Grigoras et al., 2010a). FBNYV and PNYDV were propagated by aphid transmission using $A$. pisum from infected plants. For FBNSV, agrobacteria containing infectious clones constructed as described in (Grigoras et al., 2009) (kindly provided by Prof. Stephane Blanc) were agro-inoculated to $V$. faba plants as described in (Timchenko et al., 2006).

For all three viruses, infection was confirmed by ELISA (Gaafar et al., 2016; Gaafar et al., 2017). However, for confirmation of FBNSV infection an additional PCR was carried out using FBNSV specific primers for DNA-R and DNA-N as described in (Grigoras et al., 2018).

\subsubsection{Electrical penetration graph (EPG) monitoring:}

Adult apterae $A$. pisum were starved for about 1 hour before the recording. Each aphid was immobilized using a vacuum-operated plate under a binocular microscope. A gold wire (insect electrode), $18 \mu \mathrm{m}$ in diameter and about $3 \mathrm{~cm}$ long, was glued to the aphid dorsum using a small drop of water-based silver glue and connected to the EPG probe (EPG Systems, Wageningen, The Netherlands). A copper wire, $0.2 \mathrm{~cm}$ in diameter and $10 \mathrm{~cm}$ long, was inserted into soil near to the plant root (plant electrode). The two electrodes were connected to an eight-channel GIGA-8 direct current amplifier (EPG Systems). The wired insect was placed on the stabilised abaxial surface of a faba bean leaf. The signal was digitized $(100 \mathrm{~Hz})$ using a DI-710 board (Dataq Instruments, Akron, $\mathrm{OH}$, USA) and analysed with Stylet+ $d$ software (EPG Systems). The experiment was carried out in an electrically grounded Faraday cage at a room temperature maintained around $22^{\circ} \mathrm{C}$. After starting the recording, the output voltage was optimized during the first probes for each channel by adjusting the plant voltage and gain (Giga manual; EPG Systems). The probing and feeding behaviours of individual aphids on faba bean plants was monitored for 9 hours.

\subsubsection{EPG parameters:}

The recorded waveforms were analysed with Assisted Analysis of Electrical Penetration Graph (A2EPG) software (Adasme-Carreño et al., 2015). For the calculation of the EPG parameters, the Excel-VBA macro prepared by Prof. Edgar Schliephake was used (Schliephake et al., 2013). Eighty-nine parameters were estimated and used for statistical analysis.

\subsubsection{Statistical analysis:}

The statistical analysis was carried out using scripts written on R software (version 3.5.1) (R Core Team, 2019). The EPG data were not normally distributed, thus non- 
parametric Kruskal-Wallis test was used for analysis. For significant different parameters, Pairwise Wilcox test was used to know which pairs of groups are different ( $P$ value adjustment method: $\mathrm{BH}$ ). Plotting was done using ggplot package.

\subsubsection{Experimental design:}

\subsubsection{Effect of nanovirus infection on the behavioural responses of $A$. pisum during} probing and feeding on faba beans:

Ten viruliferous aphids per plant were placed on 11-day old V. faba seedlings and let to feed on the plants for five days. The aphids were gently removed using water without any insecticides, and the plants were checked to confirm that no aphids (adult or nymph) were still present. Ten days after removing the aphid the plants were used for EPG experiments. Healthy control plants were sown the same time with the other plants and used for EPG experiments 26 days from sowing. At this stage, synchronised nonviruliferous adult aphids were used (with a maximum 24 hours age difference). After EPG recording, the exposed leaves were cut and used for ELISA confirmation tests. The recording was done for 9 hours, with all the four experimental parameters were present at the same time.

The four treatments in this experiment:

1- A. pisum feeding on FBNYV infected $V$. faba (I-FBNYV)

2- A. pisum feeding on PNYDV infected V. faba (I-PNYDV)

3- A. pisum feeding on V. faba pre-treated with A. pisum (H-Aphid)

4- A. pisum feeding on healthy $V$. faba without treatments (Healthy) 


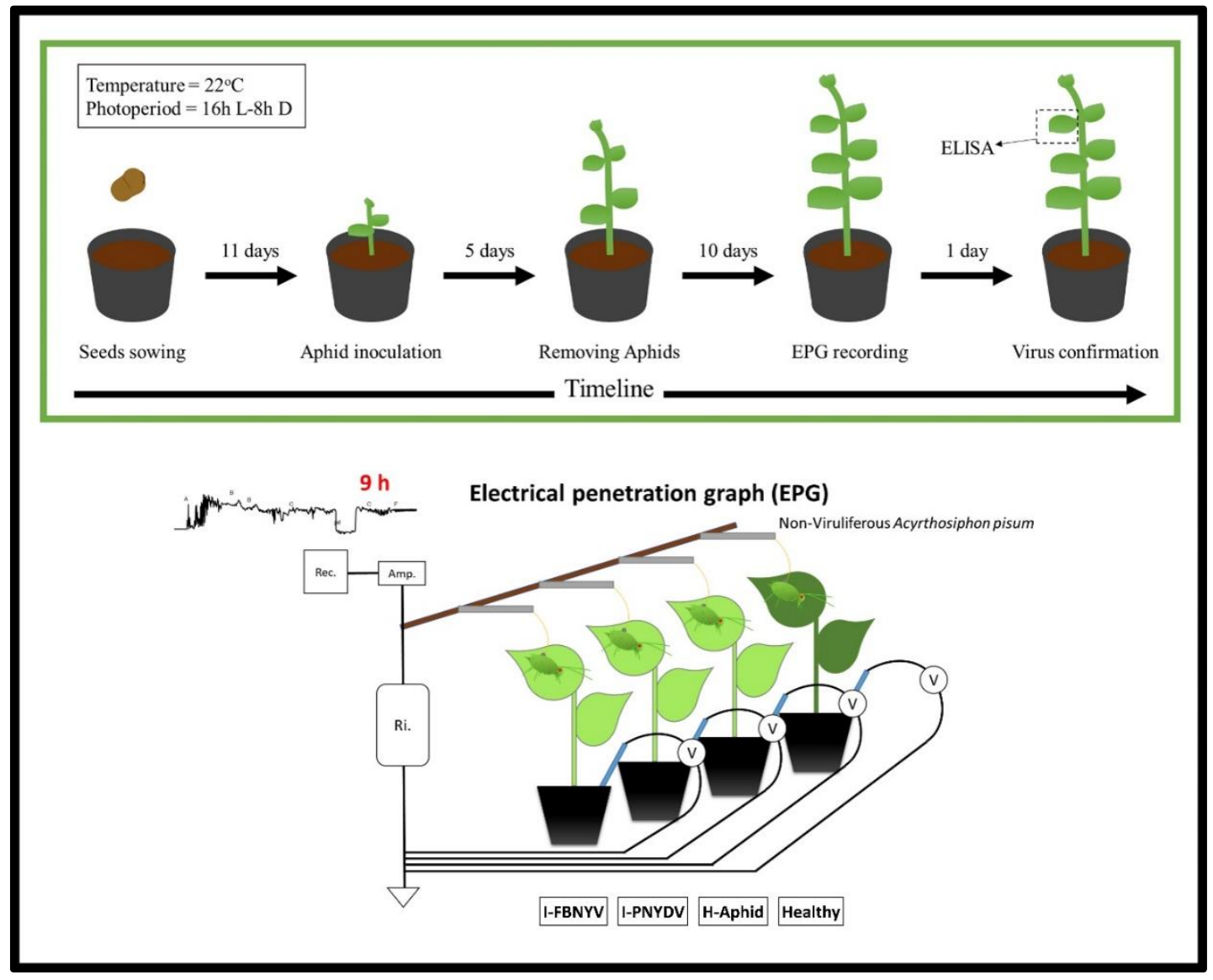

Figure 1: Experimental design to investigate the effect of nanovirus infection on probing and feeding behaviour of $A$. pisum on faba beans. I-FBNYV: FBNYV infected plants, I-PNYDV: plants infected with PNYDV, H-Aphid: plants pre-treated with aphids, and Healthy: plants without treatments.

\subsubsection{Effect of the nuclear shuttle protein (NSP) of FBNSV on the behavioural} responses of $A$. pisum during probing and feeding on faba bean:

After 11 days from seeds' sowing, the plants were agroinoculated as described before using these parameters: a) a mix of agrobacteria carrying all eight FBNSV components, b) a mix of agrobacteria carrying seven components of FBNSV (missing DNA$\mathrm{N}$ ), and c) agrobacteria not containing any FBNSV infectious clones. Fifteen days from agroinoculation, the plants were used for EPG. Healthy control plants were sown the same time with the other plants and used for EPG 26 days from sowing. Non-viruliferous adult aphids were used. After recording, the leaves where the aphid probed were cut and used for ELISA and PCR confirmation tests. The recording was done for 9 hours, with all the four experimental parameters present at the same time. 
The four treatments in this experiment:

1- A. pisum feeding on $V$. faba agroinoculated with agrobacteria containing the infectious clones of the eight components of FBNSV (I-FBNSV)

2- A. pisum feeding on $V$. faba agroinoculated with agrobacteria containing the infectious clones of the seven components of FBNSV without component DNA$\mathrm{N}(\mathrm{I}-\mathrm{FBNSV} /-\mathrm{N})$

3- A. pisum feeding on $V$. faba agroinoculated with agrobacteria without any infectious clones (H-Agro)

4- A. pisum feeding on healthy $V$. faba without treatments (Healthy)

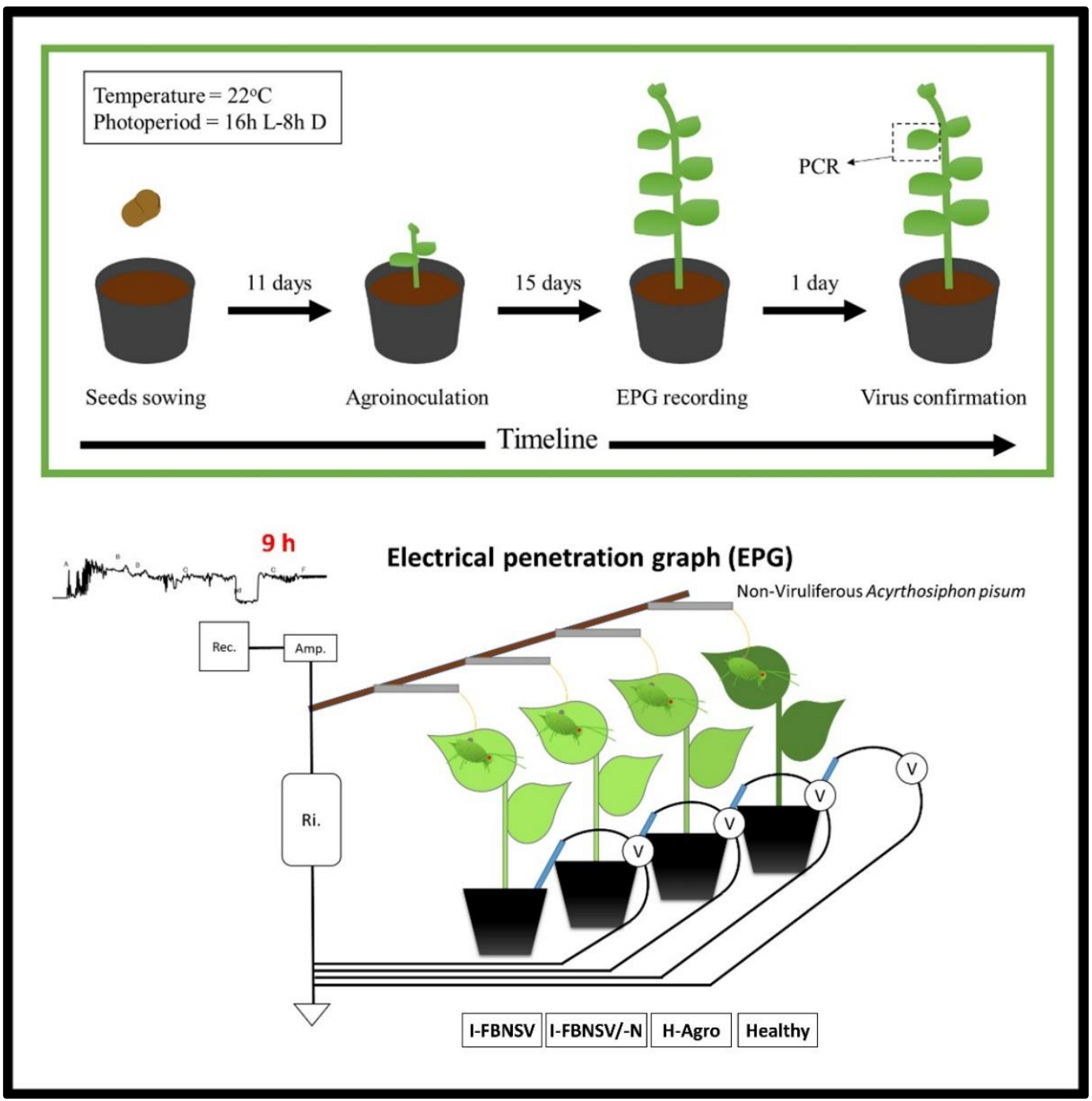

Figure 2: Graphical illustration of the experimental design for the effect of NSP absence experiment. I-FBNSV: plants agroinoculated with the eight components of FBNSV, I-FBNSV/-N: plants agroinoculated with seven components of FBNSV without DNA-N, H-Agro: plants agroinoculated with agrobacteria without infectious clones, and Healthy: plants without treatments. 


\subsection{Results}

\subsubsection{Endosymbiotic bacteria:}

PCR analyses confirmed the presence of endosymbiotic bacteria in the JKI clone of the A. pisum colony. The bacteria belonged to the genera: Buchnera, Hamiltonella and Spiroplasma.

\subsubsection{Virus-induced symptoms:}

The faba bean plants infected with FBNYV isolate AZ showed strong symptoms of top leaf carling and hardening, and the plants were severely dwarfed compared to the controls at the time of the EPG recording. The plants infected with PNYDV Drohndorf-15 showed only slight yellowing at the time of recording. In addition, the agroinoculated plants with FBNSV and FBNSV/-N showed top leaf carling and the plants were more stunted than control plants. Plants that were agroinoculated without the virus infectious clones showed no obvious symptoms.

\subsubsection{EPG probing and feeding behaviour:}

\subsubsection{Effect of nanovirus infection on the behavioural responses of $A$. pisum during} probing and feeding on faba bean:

On the non-treated healthy controls (Healthy), A. pisum spent $93.1 \%$ of the recorded time probing while the phloem activity was $67.5 \%$ (Figure 3 ). The time to first probing was $4623.8 \pm 776.7 \mathrm{sec}$ (Table 1 ). The average number of pathways (n_C) was 25.4 \pm 3.4 and the average number of intracellular punctures ( $n \_p d$ ) was $149.1 \pm 21.8$ (Table 1). The average total derailed stylet mechanics (s_F) was $1244.0 \pm 535.2 \mathrm{sec}$ (Table 1). The salivation period (S_E1) was $468.8 \pm 160.7 \mathrm{sec}$ and the time to start the first feeding (t_1E2) was $4011.9 \pm 698.5 \mathrm{sec}$ (Table 1 ). The average total duration of sustained (>10 min) phloem sap ingestion (s_sE2) was $21428.4 \pm 1611.3 \mathrm{sec}$. 


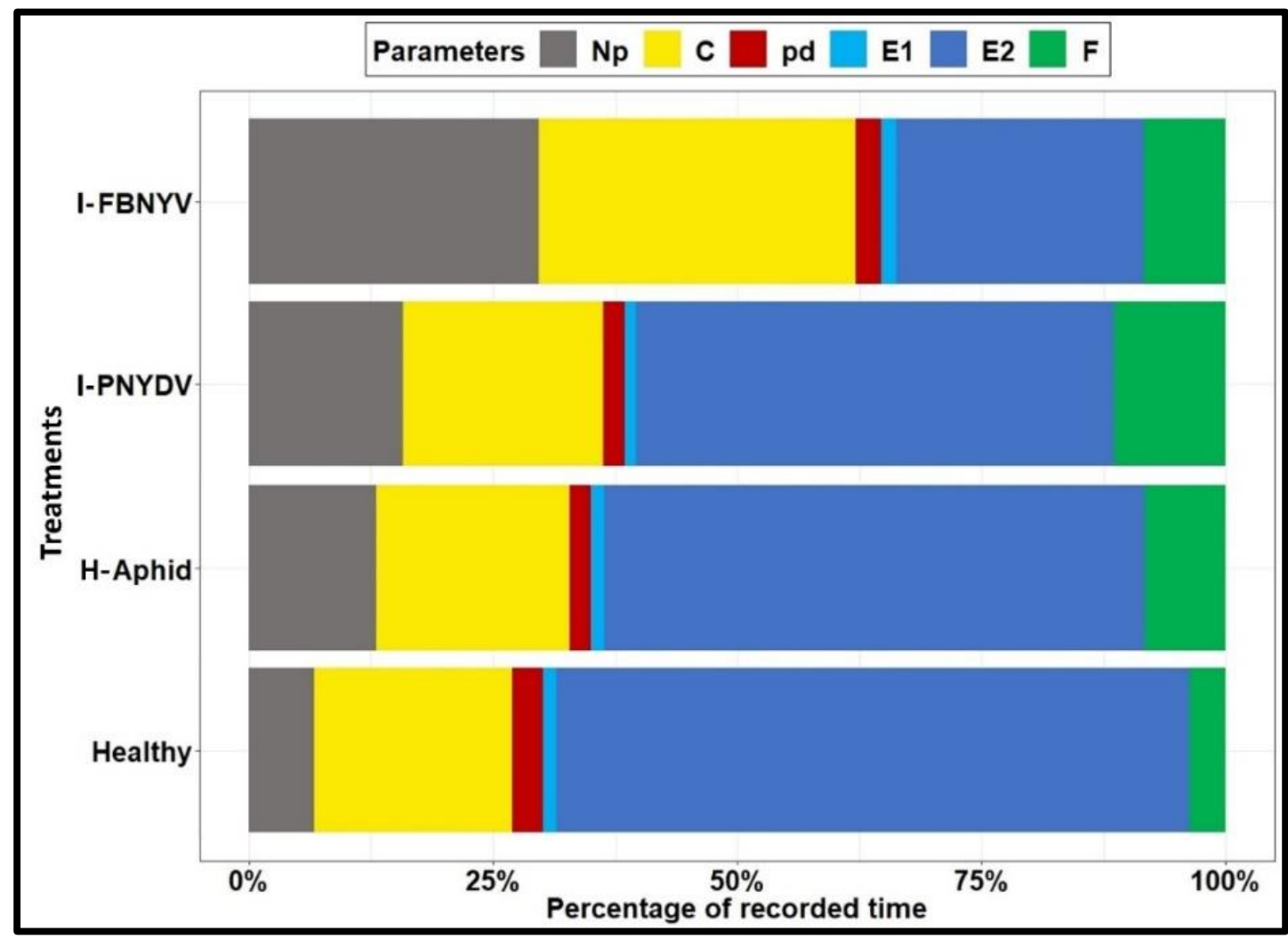

Figure 3: Percentages of the main parameters recorded for $A$. pisum feeding on FBNYV and PNYDV infected $V$. faba (I-FBNYV and I-PNYDV, respectively), aphid pre-treated healthy plants (H-Aphid) and healthy plants using EPG. The parameters are Np: non probing, C: pathway, pd: intracellular puncture, E1: salivation, E2: ingestion, F: derailed stylet mechanics. 


\begin{tabular}{|c|c|c|c|c|c|c|c|c|c|c|c|c|c|c|c|c|c|c|}
\hline $\begin{array}{l}n \\
\stackrel{n}{n} \\
\mathbb{N}\end{array}$ & $\mid \begin{array}{l}\stackrel{+}{\mathrm{N}} \\
\mathbf{m}\end{array}$ & 's & $\mid \begin{array}{l}+ \\
\end{array}$ & $i^{n}$ & I & $\begin{array}{l}n \\
m \\
N\end{array}$ & ב & 'm & $\begin{array}{l}\boldsymbol{I} \\
\\
\end{array}$ & "ח & דיב' & n's & 'n & $\mid \begin{array}{l}n \\
\text { in }\end{array}$ & $\begin{array}{l}\bar{x} \\
1 \\
z \\
0\end{array}$ & $\begin{array}{l}\frac{T}{0} \\
\frac{2}{2} \\
3 \\
\frac{10}{3} \\
\frac{10}{3}\end{array}$ & 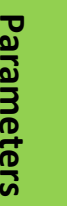 & \\
\hline 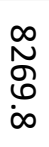 & 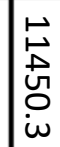 & $\begin{array}{l}\qquad \dot{\circ} \\
6 \\
6\end{array}$ & $\begin{array}{l}\vec{\circ} \\
\text { D } \\
\text { Oे } \\
0\end{array}$ & $\begin{array}{l}\infty \\
\sigma \\
\infty \\
\infty\end{array}$ & $\vec{g}$ & $\begin{array}{l}\infty \\
\stackrel{\infty}{\omega} \\
\stackrel{\sim}{\sim}\end{array}$ & ம் & $\begin{array}{l}\text { U } \\
0 \\
\dot{1}\end{array}$ & $\underset{i}{\omega}$ & $\begin{array}{l}\tilde{U} \\
\dot{O} \\
\dot{i}\end{array}$ & ம & 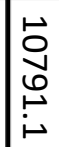 & $\begin{array}{l}n \\
\infty \\
\sigma\end{array}$ & $\begin{array}{l}\mathscr{1} \\
\infty \\
\infty \\
\infty \\
\not \triangleright\end{array}$ & 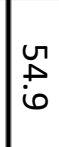 & 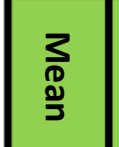 & \multirow{2}{*}{$\stackrel{3}{\Delta}$} & \\
\hline 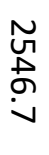 & $\begin{array}{l}\tilde{N} \\
\tilde{N} \\
\stackrel{N}{N}\end{array}$ & $\stackrel{\stackrel{\bullet}{\circ}}{\circ}$ & $\begin{array}{l}\tilde{\sigma} \\
\tilde{\sigma} \\
\tilde{\sigma} \\
\dot{v}\end{array}$ & $\underset{\tilde{\omega}}{\tilde{N}}$ & $\begin{array}{l}\tilde{O} \\
0 \\
0\end{array}$ & $\begin{array}{l}\tilde{N} \\
\mathbb{N} \\
\tilde{\omega}\end{array}$ & $\stackrel{\circ}{\circ}$ & 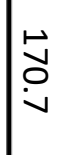 & ir & $\underset{w}{w}$ & $\stackrel{\circ}{\circ}$ & $\begin{array}{l}\vec{w} \\
\tilde{\sigma} \\
\infty \\
\infty\end{array}$ & $\begin{array}{l}a \\
\text { in }\end{array}$ & 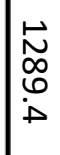 & in & 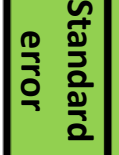 & & $\sum$ \\
\hline $\begin{array}{l}\vec{\sigma} \\
\stackrel{\vec{\theta}}{\mathbf{i}}\end{array}$ & 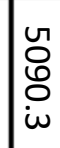 & ज़ & 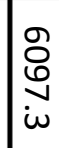 & 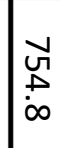 & $\begin{array}{l}\overrightarrow{\mid} \\
\text { ŭ } \\
\sigma\end{array}$ & $\begin{array}{l}\hat{\sigma} \\
\tilde{N} \\
\tilde{\sigma} \\
\dot{\omega}\end{array}$ & w & $\begin{array}{l}w \\
\stackrel{p}{ } \\
\sigma\end{array}$ & $\stackrel{\vec{r}}{\dot{r}}$ & $\begin{array}{l}w \\
y \\
\infty \\
\sigma\end{array}$ & ம் & $\begin{array}{l}\mathscr{\sigma} \\
\text { Dे } \\
\text { in }\end{array}$ & $\begin{array}{l}\omega \\
\infty \\
\varnothing \\
\end{array}$ & $\begin{array}{l}\mathcal{u} \\
\mathcal{N} \\
\stackrel{O}{V}\end{array}$ & $\underset{+}{\infty}$ & $\begin{array}{l}3 \\
\mathbb{1} \\
\frac{N}{3}\end{array}$ & & $\bar{T}$ \\
\hline 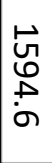 & 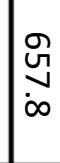 & $\stackrel{\infty}{\bullet}$ & $\begin{array}{l}\infty \\
\omega \\
\sigma \\
0 \\
0\end{array}$ & $\begin{array}{l}\infty \\
\text { in } \\
\text { in }\end{array}$ & $\stackrel{\bullet}{\stackrel{\bullet}{\circ}}$ & 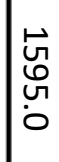 & i & $\begin{array}{l}\checkmark \\
6\end{array}$ & $\stackrel{\circ}{\circ}$ & 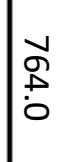 & $\stackrel{\circ}{\circ}$ & $\underset{\sim}{\tilde{\omega}}$ & $\stackrel{\vec{c}}{\vec{b}}$ & 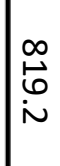 & $\stackrel{i}{\leftarrow}$ & 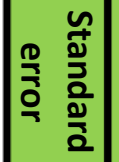 & & खू \\
\hline$\stackrel{\stackrel{\infty}{\infty}}{\underset{\infty}{\infty}}$ & 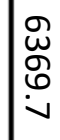 & 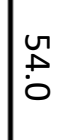 & 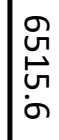 & $\begin{array}{l}6 \\
\dot{b} \\
\dot{\sim}\end{array}$ & $\stackrel{\stackrel{E}{\sim}}{\stackrel{\sim}{\sim}}$ & $\begin{array}{l}\vec{\infty} \\
\tilde{\omega} \\
\omega \\
\infty \\
\infty\end{array}$ & 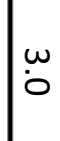 & 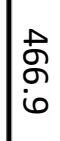 & $\stackrel{\vec{p}}{\stackrel{5}{ }}$ & 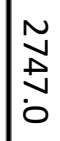 & $\stackrel{\bullet}{\triangleright}$ & 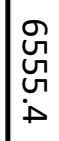 & $\begin{array}{l}w \\
\sigma\end{array}$ & 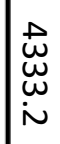 & $\underset{\infty}{\omega}$ & $\begin{array}{l}3 \\
\mathbb{1} \\
\frac{N}{5}\end{array}$ & & $T$ \\
\hline $\begin{array}{l}\tilde{v} \\
\text { V }\end{array}$ & $\begin{array}{l}\infty \\
\infty \\
\text { aे }\end{array}$ & ir & $\begin{array}{l}\stackrel{0}{\circ} \\
\stackrel{0}{0} \\
\infty\end{array}$ & $\begin{array}{l}\vec{s} \\
0 \\
\sigma\end{array}$ & $\stackrel{\tilde{f}}{\hat{i r}}$ & 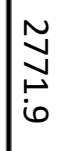 & ó & $\begin{array}{l}\overrightarrow{0} \\
\infty \\
0\end{array}$ & $\stackrel{\oplus}{\sim}$ & $\begin{array}{l}0 \\
\sigma \\
\dot{\sigma} \\
\dot{v}\end{array}$ & : & 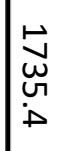 & $\underset{\sim}{\stackrel{\sim}{N}}$ & 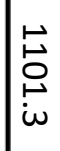 & $\underset{\triangleright}{\stackrel{\circ}{\circ}}$ & 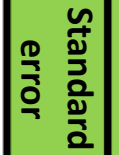 & & 음 \\
\hline 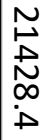 & $\mid \begin{array}{c}\stackrel{\bullet}{\circ} \\
\stackrel{\bullet}{\bullet} \\
\stackrel{0}{0}\end{array}$ & $\begin{array}{l}\infty \\
\infty \\
0\end{array}$ & $\begin{array}{l}\stackrel{p}{\sim} \\
\omega \\
\infty \\
\infty\end{array}$ & $\begin{array}{l}10 \\
0 \\
0 \\
6\end{array}$ & $\mid \begin{array}{l}\vec{E} \\
\dot{\varphi} \\
i\end{array}$ & $\begin{array}{l}\tilde{N} \\
\hat{\sigma} \\
\tilde{\sigma} \\
\sigma\end{array}$ & $\underset{\sigma}{\omega}$ & $\begin{array}{l}\infty \\
\infty \\
\infty \\
\infty\end{array}$ & $\dot{\forall}$ & $\begin{array}{l}\stackrel{\tilde{N}}{\stackrel{+}{+}} \\
\dot{0}\end{array}$ & 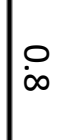 & 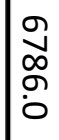 & $\underset{\sim}{\sim}$ & $\begin{array}{l}\tilde{N} \\
\stackrel{N}{*} \\
\dot{v}\end{array}$ & $\stackrel{\tilde{\varphi}}{\sigma}$ & $\begin{array}{l}3 \\
\frac{1}{5} \\
\frac{N}{5}\end{array}$ & \multirow[b]{2}{*}{$\vartheta$} & \multirow{2}{*}{$\mid \begin{array}{l}z \\
\frac{2}{2} \\
\frac{2}{z} \\
z\end{array}$} \\
\hline 宫 & $\begin{array}{l}\mathscr{0} \\
\dot{\infty} \\
\text { ir }\end{array}$ & $\begin{array}{l}\vec{\sigma} \\
\infty\end{array}$ & $\begin{array}{l}y \\
y \\
y\end{array}$ & $\begin{array}{l}\hat{\sigma} \\
6 \\
\sigma\end{array}$ & $\stackrel{\sim}{\stackrel{\sim}{\infty}}$ & $\begin{array}{l}\hat{v} \\
N \\
N \\
\tilde{\omega}\end{array}$ & ó & 홍 & 우 & $\begin{array}{l}\tilde{w} \\
\stackrel{\sim}{\sim} \\
\sim\end{array}$ & ir & $\begin{array}{l}\tilde{L} \\
\tilde{N} \\
\tilde{\omega}\end{array}$ & $\underset{\Delta}{\omega}$ & $\begin{array}{l}\overrightarrow{\vec{t}} \\
\dot{0}\end{array}$ & $\underset{\infty}{\infty}$ & 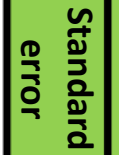 & & \\
\hline 영 & 웅 & $\begin{array}{l}\stackrel{P}{\circ} \\
\stackrel{1}{\ominus}\end{array}$ & ○ & $\underset{\phi}{\underset{\phi}{\omega}}$ & 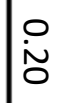 & ○ & 웅 & 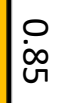 & $\begin{array}{l}\stackrel{O}{\text { N }} \\
\text { in }\end{array}$ & 임 & 음 & 임 & ○ & ○ & ○ & 离 & & \\
\hline
\end{tabular}

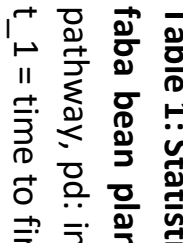

홓

三

号 亏ั

命

은 웡 훙

辛 血

ล

品思 㐘

य <

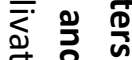

옥 꾼 온

정

鬲

员 훈 웅

○

ํํำ ํํำ

끄음

울 ₹

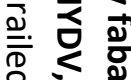

눙

垈蛋

事

了

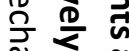

กิำ ํㅡㄹ

于

II 产 产

夌兽

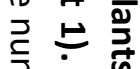

궁 구 웅

응

요월

in 突 \%

11 )

粂票

空 ?

윽 농.

三

ป 음

은 응 홍

은 을 훈.

들 으

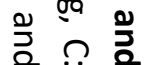


On plants pre-treated with aphids (H-Aphid), the EPG parameters were not significantly different from those on the Healthy plants. The probing time of $A$. pisum was $86.6 \%$ and phloem activity was recorded (57.5\%) (Figure 3 ). The $n \_C$ was $37.6 \pm 10.2, n \_p d$ was $112.2 \pm 24.5$, and the S_F was $2747.0 \pm 916.5 \mathrm{sec}$ (Table 1). The S_E1 was $466.9 \pm$ $108.6 \mathrm{sec}$ and the t_1E2 took $6369.7 \pm 987.6 \mathrm{sec}$ on average (Table 1). The s_sE2 was $18128 \pm 2750.7 \mathrm{sec}$.

When $A$. pisum feed on $V$. faba infected with FBNYV (I-FBNYV), the EPG parameters were significantly different in many cases than those recorded when feeding on healthy plants H-Aphid and the Healthy. The aphids probed and fed on I-FBNYV for $69.5 \%$ of the recording time ( 9 hours) on average, shorter than the other treatments and spent shorter time on the phloem, only $26.4 \%$ of the recording time (Figure 3 ). Once the stylet reached the phloem, the salivation was not significantly different from the other three treatments. Interestingly, the t_1E2 was significantly longer than the other treatments $11450.3 \pm 2250.2$ seconds (Table 1 ). Outside the phloem, the $n \_C$ was significantly different with the Healthy but not with $\mathrm{H}$-Aphid (Table 1). The derailed stylet mechanics was also not significantly different between the different treatments. The s_sE2 (8269.8 $\pm 2546.7 \mathrm{sec})$ also significantly decreased.

On PNYDV-infected plants (I-PNYDV), the EPG parameters were significantly different in several phloem related activities compared to I-FBNYV and Healthy plants but did not differ from the H-Aphid. A. pisum's probing time was $83.8 \%$ significantly higher than I-FBNYV but lower than the $\mathrm{H}$ plants, but not significantly different from H-Aphid plants (Figure 3). The phloem activity on the I-PNYDV was $50.7 \%$ of the recorded time (Figure 3) also significantly higher than the I-FBNYV, lower than the Healthy, and not different from $\mathrm{H}$-Aphid. The salivation activity was not significantly different between IPNYDV and the other treatments. The t_1E2 (5090.3 \pm 657.8 seconds) was significantly shorter than I-FBNYV but not significantly different form the controls (Table 1). The n_C was significantly lower than I-FBNYV but not different from both controls. The S_SE2 was $16100.2 \pm 1594.6 \mathrm{sec}$, significantly higher than I-FBNYV but not different from Healthy and H-Aphid.

\subsubsection{Effect of the nuclear shuttle protein (NSP) of FBNSV on the behavioural} responses of $A$. pisum during probing and feeding on faba bean:

In general, the EPG parameters for $A$. pisum feeding on the four different $V$. faba plants were not significantly different (Table 2). A. pisum probed and fed on average on Healthy plants for $81.1 \%$ of the recording time (Figure 4 ). The aphids spent $45.4 \%$ phloem feeding and $1.9 \%$ salivating on $\mathrm{H}$ plants (Figure 4). The $\mathrm{n} \_\mathrm{C}, \mathrm{n} \_\mathrm{pd}$ and $\mathrm{t} \_1 \mathrm{E} 2$ were $36.1 \pm$ $5.6,193.8 \pm 26.2$ and $9286.4 \pm 1771.9 \mathrm{sec}$, respectively (Table 4). The $n \_E 1$ and $n \_E 2$ of $A$. pisum were $6.0 \pm 0.9$ and $3.4 \pm 0.6$, respectively. The $n_{-} F$ and S_F were $0.5 \pm 0.3$ and 981.4 $\pm 482.1 \mathrm{sec}$, respectively. The s_sE2 was $14395.3 \pm 2037.3 \mathrm{sec}$. 


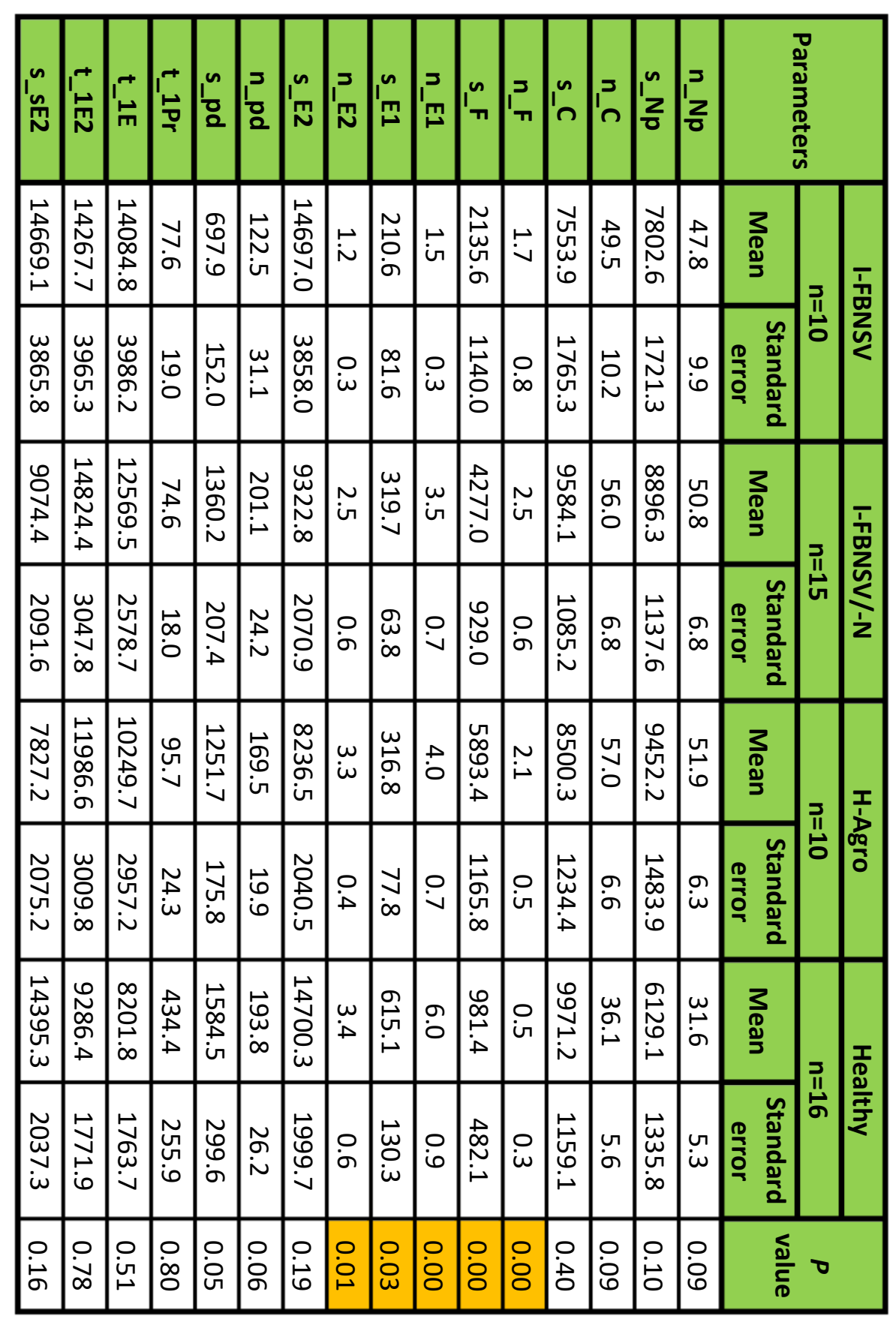

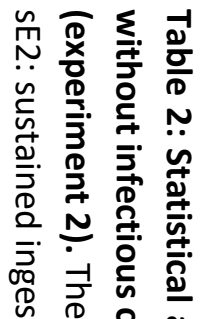

웅 월 응 产

ว

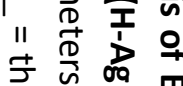

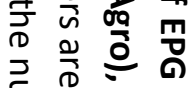

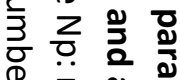

웅 웅 ㅁํㅇ

is 흑 응 준

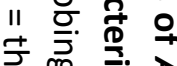

宽骂 啇

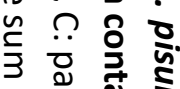

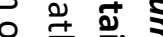

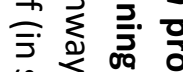

ज $\leqslant \infty$

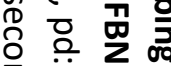

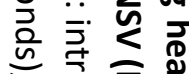

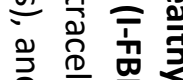

믈

$\mapsto$ ำ

I․ 흥 을 융

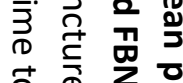

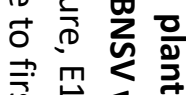

ज约

ज刨 容

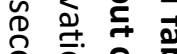

을 을 웧

은 塄

$\stackrel{ }{\circ}$ 을

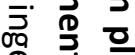

号 号

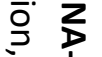

पर 2

응

离. 盛

位

范立这

强

ํํㄱ 융. ำ

ขै 음

등 응

을 总 


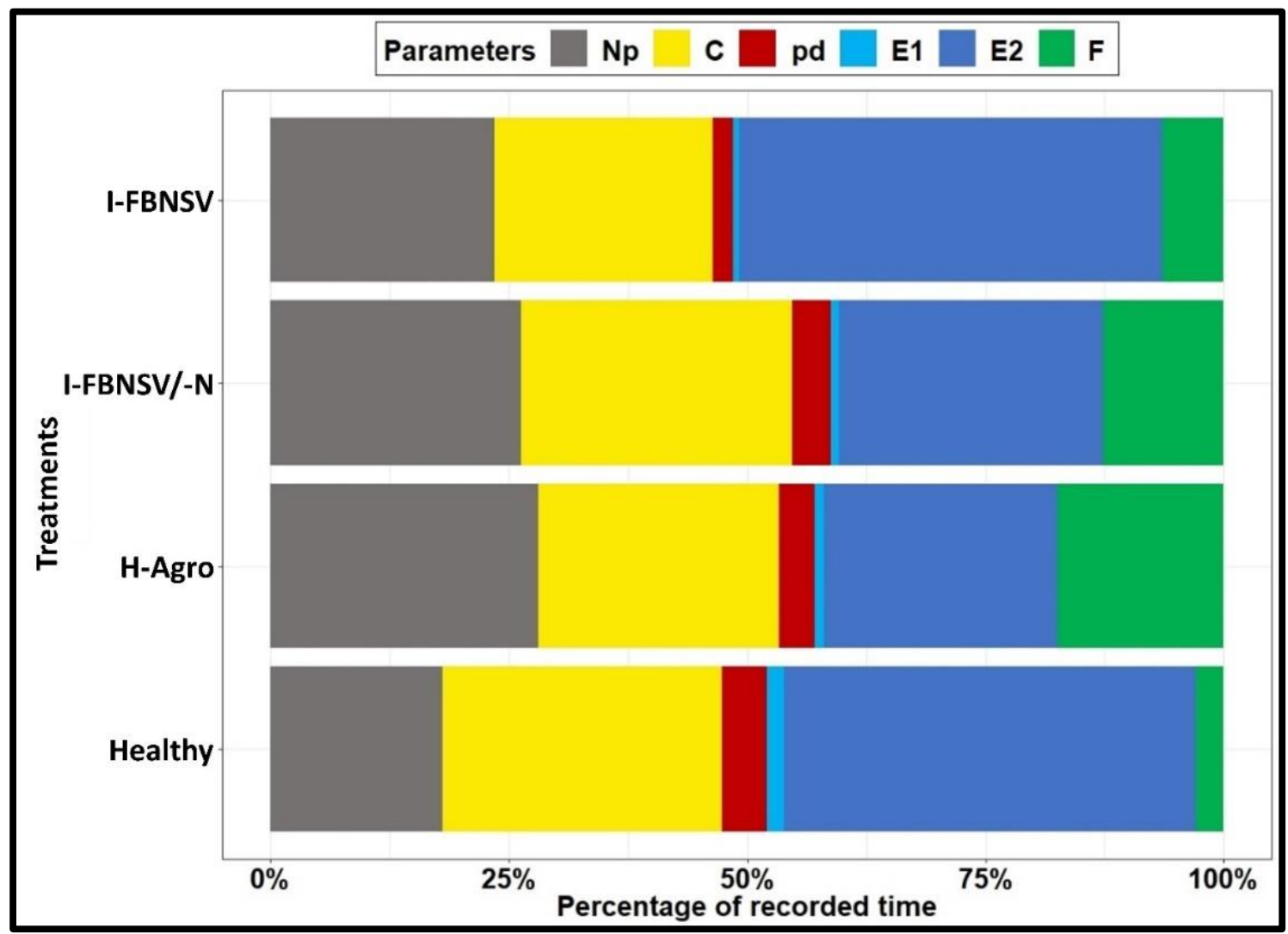

Figure 4: Percentages of the main parameters recorded for A. pisum feeding on I-FBNSV and IFBNSV/-N infected $V$. faba, agrobacteria pre-treated healthy plants (H-Agro), and healthy plants using EPG. The parameters are Np: non probing, C: pathway, pd: intracellular puncture, E1: salivation, E2: ingestion, and F: derailed stylet mechanics.

The aphids spent $70.8 \%$ of the recorded time probing on agrobacteria treated plants (H-Agro), which was not significantly different from on Healthy control (Figure 4). A. pisum spent $25.4 \%$ phloem feeding on $\mathrm{H}$-Agro plants and $0.9 \%$ salivation. The $\mathrm{n}$ - $\mathrm{C}$ was $57.0 \pm 6.6$, the $n \_p d$ was $169.5 \pm 19.9$ and t_1E2 was $11986.6 \pm 3009.8 \mathrm{sec}$ (Table 4). The n_E1 and n_E2 were $4.0 \pm 0.7$ and $3.3 \pm 0.4$, respectively. The $\mathrm{n} \_\mathrm{F}$ and S_F of $A$. pisum on $\mathrm{H}$-Agro were significantly higher than from on Healthy plants with $2.1 \pm 0.5$ and $5893.4 \pm$ $1165.8 \mathrm{sec}$, respectively (Table 4). The s_sE2 was $7827.2 \pm 2075.2 \mathrm{sec}$, not significantly different from on the Healthy.

A. pisum probed and fed on FBNSV-infected plants (I-FBNSV) for $75.9 \%$ of the recording time on average. On average, the aphids spent less than $50 \%$ of the recording time ( 9 hours) on the phloem of I-FBNSV with $45.4 \%$ ingestion and $0.6 \%$ salivation (Figure 4). The $n \_C$ on I-FBNSV was $49.5 \pm 10.2$ and the $n \_p d$ was $122.5 \pm 31.1$ (Table 2). The $n \_F$ and S_F ( $2.5 \pm 0.6$ and $4277 \pm 929$, respectively) were significantly different from the $\mathrm{H}$ Agro, but not from the Healthy. The t_1E2 was $14267.7 \pm 3965.3 \mathrm{sec}$ (Table 2). Although the s_E2 $(14697 \pm 3858 \mathrm{sec})$ was not significantly different from the other treatments, the n_E2 was $1.2 \pm 0.3$ significantly lower than on H-Agro and on Healthy plants. The S_sE2 was $14669.1 \pm 3865.8 \mathrm{sec}$. 
On I-FBNSV/-N plants, the aphids spent $72.5 \%$ of the recorded time probing, not significantly different from the other treatments (Figure 4). Aphids spent only $28.8 \%$ of the recording time phloem feeding and $0.9 \%$ salivating (Figure 4). The $n \_C$ was $56.0 \pm 6.8$, the $n \_p d$ was $201.1 \pm 24.2$ and the t_1E2 was $14824.4 \pm 3047.8 \mathrm{sec}$ (Table 4). None of the phloem activities were significantly different from the other treatments. The $n_{-} F$ and s_F (2.5 \pm 0.6 and $4277 \pm 929 \mathrm{sec}$, respectively) were significantly higher than on Healthy plants.

\subsection{Discussion}

\subsubsection{Endosymbionts of the JKI A. pisum clone}

There is evidence that microbial symbioses influence aphid-plant interactions (Frago et al., 2012). It was therefore necessary to identify the symbionts in the A. pisum clone JKI used in this study. In the JKI A. pisum clone, the essential intracellular symbiotic bacterium Buchnera was detected. The primary symbiont (P-symbiont) Buchnera is present in almost all aphids in the cytoplasm of their mycetocytes (or bacteriocytes) in their abdomen (Buchner, 1965; Baumann et al., 1995). Buchnera is tightly restricted to the aphid's body cavity. Buchnera symbionts and their aphids are intimately mutualistic (Houk \& Griffiths, 1980; Ishikawa \& Yamaji, 1985; Ohtaka, 1991). It was found that the Buchnera cannot survive when removed from their host cells, and that aphids suffer sterility or even death when deprived of Buchnera (Houk \& Griffiths, 1980; Ishikawa \& Yamaji, 1985; Ohtaka, 1991). Thus, it is not surprising to detect such intimately mutualistic symbionts in the JKI aphid clone.

In addition to Buchnera, two facultative secondary symbionts (S-symbiont) were detected in the aphids. These are a pea aphid Bemisia-type symbiont (PABS) "Hamiltonella defensa", and the Spiroplasma symbiont (Fukatsu et al., 2001; Moran et al., 2005). PABS was detected before in both the gut and the ovaries of aphids; in contrast with the primary symbiont Buchnera which is restricted to cytoplasm of the bacteriocytes (Munson et al., 1991; Wilkinson \& Douglas, 1998). The removal of $H$. defensa from $A$. pisum led to decrease in the fecundity rate by about 20\% (McLean et al., 2011). Moreover, H. defensainfected $A$. craccivora exhibiting depressed probing behaviour when tested with EPG (Angelella et al., 2018). Spiroplasma symbionts can negatively affect the growth, longevity and reproduction of $A$. pisum (Fukatsu et al., 2001). No information about the interaction between Spiroplasma sp. and plant viruses is available. Therefore, the endosymbiotic organization of the JKI clone of $A$. pisum is Buchnera (P-symbiont) and both Hamiltonella and Spiroplasma (S-symbiont). The presence of other genera in the clone is still possible, thus for additional confirmation, we suggest using high throughput sequencing (HTS) to detect all the endosymbionts in the clone.

The importance of endosymbiotic activity for virus transmission has been described for few systems. For example, virus particles of potato leafroll virus (PLRV; genus: Polerovirus, family: Luteoviridae), also a circulative, persistently-transmitted virus, 
bind to the chaperonin protein "GroEL" produced by Buchnera aphidicola. This binding seems to protect the virus particles from degradation in the aphid's haemocoel (van den Heuvel et al., 1994). Feeding of antibiotics to Myzus persicae nymphs prior PLRV acquisition reduced the virus transmission by more than $70 \%$ (van den Heuvel et al., 1994). A study on tomato yellow leaf curl virus (TYLCV) (genus: Begomovirus, family: Geminiviridae) using yeast two-hybrid and protein pulldown assays showed that GroEL protein produced by Hamiltonella interacts with the coat protein of TYLCV and facilitates the virus transmission (Gottlieb et al., 2010). The virus particles binding to the GroEL produced by symbionts seems to protect the viruses' particles from degradation in the aphid's haemocoel (van den Heuvel et al., 1994; Gottlieb et al., 2010). In case of banana bunchy top virus (genus: Babuvirus, family Nanoviridae), there was no interaction of virus particles with Buchnera GroEL (Watanabe et al., 2013) and there is currently no evidence that nanoviruses interact with their vectors' endosymbionts. It is suggested that nanovirids translocate in large clusters of virions in aphid (Watanabe \& Bressan, 2013; Vetten et al., 2016). This might protect the individual particle from degradation and might be the reason that prevents the virions interaction with the GroEL (Watanabe et al., 2013). The biological effect of the S-symbionts and their interactions with nanoviruses are unknown and it is of interest to investigate these interactions.

\subsubsection{Experiment 1: Effect of nanovirus infection on the behavioural responses of $A$.} pisum during probing and feeding on faba bean:

In this study, we used EPG to evaluate whether the infection of nanoviruses to faba beans can change the feeding and probing behaviours of $A$. pisum.

\subsubsection{The behaviour of $A$. pisum changed on FBNYV and PNYDV infected faba beans:}

At the time of recording, FBNYV infected plants were dwarfed with top leaf carling and hardening. As the aphid feed on the lower surface of the top leaves with these severe symptoms, these cytological changes of the host plant might explain the change in the feeding and probing behaviour of $A$. pisum. The probing period on I-FBNYV was significantly shorter than on other treatments and aphids spent less time on phloem feeding (26.4\% of the recording time). Persistently transmitted plant viruses such as nanoviruses are restricted to the phloem tissue. Thus, early phloem sap ingestion (E2) on virus-infected plants will enhance transmission of phloem-restricted viruses, as aphids will acquire the virus faster and potentially with longer acquisition times, therefore allowing the acquisition of all viral components and helper factors need for successful virus transmission. However, the time to start the first feeding was significantly longer than the other treatments and the total duration of sustained phloem sap ingestion also decreased. The period before the first phloem activity is determined by epidermal, mesophyll, general vascular, and early phloem factors (Schwarzkopf et al., 2013). The 
significantly longer t_1E2 on FBNYV-infected plants suggests increased resistance to aphids in these tissues. Additionally, an increased number of probing was detected. From these, the manipulation of FBNYV to the infected plants appeared to be less attractive to A. pisum.

In contrast to FBNYV infected plants, the symptoms induced by PNYDV on infected plants were mild (slight yellowing compared to the healthy plants). Yet, the probing time of $A$. pisum was significantly higher than I-FBNYV and lower than the Healthy plants and it was not different from $\mathrm{H}$-Aphid plants. The same results were obtained for the phloem activity. We also observed at a later stage of the disease, that infected plants with PNYDV isolate Drohndorf-15 showed top leaf dwarfing, leaf curling and whole plant yellowing. We assume that at a later recording time with the disease development, the effect of PNYDV on the feeding and probing behaviours of $A$. pisum will increase.

These results contradict previous studies on other circulative viruses i.e., PLRV and tomato yellow leaf curl virus (TYLCV), where virus infections reduced the resistance and increased the feeding and probing behaviours (Alvarez et al., 2007; Liu et al., 2013).

\subsubsection{Aphid behaviours on aphid pre-treated plants were not different from those on healthy and PNYDV infected plants:}

Aphid infestation is known to induce local and systemic changes to plant consequently the plants develop defences to limit the damage caused by aphid (Moran \& Thompson, 2001; Cooper \& Goggin, 2005; Thompson \& Goggin, 2006). Moreover, previous infestation could affect subsequent aphid performance positively or negatively. For example, Aphis gossypii pre-infestation of cotton plants decreased recolonization by aphids (Wool \& Hales, 1996). On the other hand, M. persicae pre-infestation led to an improvement of subsequent same aphid species performance (Sauge et al., 2002). To compare whether the aphid inoculation had effects on any change in behaviour of $A$. pisum on nanoviruses infected plants, additional control of plants pre-treated with aphid was also tested. There were no major differences between healthy untreated plants and aphid pre-treated plants. It was only different from I-FBNYV as other treatments with higher phloem activity and faster feeding starting time.

These behavioural changes can be correlated to the visual symptoms of the virus disease. This is in consistence with previous finding where $M$. persicae only exhibit differences in feeding behaviour on PLRV-infected potato plants after the disease symptoms were observed (Alvarez et al., 2007). To confirm this hypothesis, additional studies are required to determine the structural and metabolic changes of nanoviruses infected plants during the development of the disease symptoms. Whether this is due to a change in the phloem sap quality or the structure of the plant tissues remains unclear as there is no study on the effect of nanovirus infection on the structure and metabolic components of the host plant. 


\subsubsection{Experiment 2: Effect of NSP on the behavioural responses of $A$. pisum during probing and feeding on faba bean:}

In general, the EPG parameters for $A$. pisum feeding on the four different $V$. faba plants were not significantly different (Table 2). Our results showed no differences in the behaviours of $A$. pisum's on FBNSV infected plants or FBNSV missing component DNA-N infected plants. Although the NSP produced by DNA-N is required for nanoviruses transmission, our results suggest that it does not affect the aphid feeding behaviour. $A$ previous study showed that DNA-N is not essential for symptoms development, and the combination of the other seven DNAs was still able to cause the disease symptoms (Timchenko et al., 2006). Surprisingly, the aphid behaviours on I-FBNSV were not different from those on $\mathrm{H}$ plants. The only difference was the number of salivations and ingestions where they were significantly lower on I-FBNSV.

The derailed stylet mechanics (F) on Healthy and I-FBNSV plants were significantly different from I-FBNSV/-N and H-Agro. The derailed stylet occurs when a stylet protruded much further than the other three stylets thus loses the bundle formation which occurs at low frequency (Tjallingii, 1988). It is suggested that the intercellular structural composition is responsible for such derailments (Tjallingii, 1988). Here, an increase in derailing of the stylet with the presence of agrobacteria, although in case of I-FBNSV the virus infection seems to reduce such effect. This could be due to the presence of the agrobacteria in the plants. As there is no study on the effect of agrobacteria on the aphid behaviours, suggesting adding an extra control with infiltration buffer and no agrobacteria, or using plants from successive aphid transmissions to reduce the effect of the agroinoculation.

It was important to identify and characterise the genomes of the viruses in the study, as the presence of different components and any of the satellite DNAs can affect the virus disease severity and the virus transmission. Previous study on FBNYV suggested that the presence of faba bean necrotic yellows C11 alphasatellite cause reduction in infected plants due to competition between the different components on the resources, encapsidation and movement (Timchenko et al., 2006). The isolate of FBNYV in this study contains in addition to the eight components, an alphasatellite i.e., faba bean necrotic yellows C1 alphasatellite, while both PNYDV and FBNSV each contains only their eight components. Whether there is an effect "positive or negative" for the alphasatellite on the severity of the nanoviruses diseases or on their aphid vectors, and consequently the aphid behaviour, is currently unknown.

The effect of the plant variety and whether the aphid behaviour will be different from a variety to another. Comparing the Healthy plants in both experiments showed the probing time was significantly different between both experiments. The reason for that is unknown as we used the same growing conditions for both experiments. Other aphid biotypes should be considered in future testing. Additional studies will be required to 
determine the structural and metabolic changes of nanoviruses infected plants during the development of the disease symptoms to confirm the relation between the change in the behaviour and symptoms. Suggesting studying the dynamic of aphid behaviour on plants with series of infection periods. Therefore, this study needs follow up with further investigations to confirm the findings. 


\section{Chapter 7: General discussion}

\subsection{Part 1: Plant virus identification}

A prerequisite to the control of a plant viral disease is the proper detection and identification of its causal agent. Thus, a sensitive and reliable detection is crucial for plant protection. To identify the aetiology of a viral disease, there are different diagnostics tools used e.g., electron microscopy (EM), serology-based methods such as enzyme linked immunosorbent assays (ELISA), molecular biology-based methods such as polymerase chain reaction (PCR) and high throughput sequencing (HTS).

\subsubsection{Disease aetiology of several plants using conventional and HTS methods}

In a legume survey, several viruses were detected in many plant samples from Germany and Austria in 2016 using conventional methods (PCR and ELISA) ((Gaafar et al., 2016), Chapter 2). We could identify pea enation mosaic virus (PEMV) as the predominant virus followed by pea necrotic yellow dwarf virus (PNYDV). Viruses from other genera e.g., potyviruses, poleroviruses and luteoviruses could be also detected by ELISA; however, the exact virus species could not be confirmed. The antibodies used for ELISA were preselected for the commonly known legume viruses. Thus, we could not confirm whether other viruses were present in the samples or not, especially when several symptomatic samples tested negative with ELISA.

Additionally, in two samples from the Netherlands, we could detect by ELISA and PCR the presence of PNYDV for the first time ((Gaafar et al., 2017), Chapter 2). We could confirm the presence of the eight genomic components of PNYDV by PCR amplification of each component, cloning in Escherichia coli, extracting the plasmids followed by Sanger sequencing. This approach was laborious and time consuming. Moreover, we could not confirm this way whether there were PNYDV-associated alphasatellites present or not.

High throughput sequencing (HTS) gave us the ability to sequence all the nucleic acids present in a given sample (Adams \& Fox, 2016). Using HTS, we were able to detect the full genomes of different PNYDV isolates from five samples from Denmark, and also we detected their associated alphasatellites ((Gaafar et al., 2018a), Chapter 2).

Other legume and vegetable samples were also analysed by conventional methods and HTS. Using EM and ELISA, we were able to identify viruses from different families and genera. However, without the presence of species-specific antibodies, we could not confirm the virus species and in some cases the virus genus. For example, using immunosorbent electron microscopy (ISEM) and ELISA, we detected the presence of turnip crinkle virus (TCV) in wild garlic mustard ((Gaafar \& Ziebell, 2019a), Chapter 2). Using HTS, a new strain of TCV was identified as being a highly divergent sequence (Rochon et al., 2012). Such divergence could not be detected by ISEM or ELISA. In a similar 
case we investigated a new nepovirus in a caraway sample where nepoviruses-specific antibodies developed against a divergent strain of cherry leafroll virus (CLRV) infecting carrot (unpublished) was used in ELISA and ISEM, and confirmed the presence of a nepovirus but could not identify the virus species ((Gaafar et al., 2019f), Chapter 2). Using HTS, we identified the presence of a novel nepovirus, tentatively called caraway yellows virus (CawYV).

Also, we could detect the presence of rhabdovirus-like particles in tomato samples from Germany, alfalfa and black medic samples from Austria. Physostigia chlorotic mottle virus (PhCMoV; a nucleorhabdovirus) in the tomato sample and a novel nucleorhabdovirus, tentatively called alfalfa associated nucleorhabdovirus (AaNV) in alfalfa sample were identified using HTS, confirming the findings of EM ((Gaafar et al., 2018b; Gaafar et al., 2019d), Chapter 2). However, in the black medic sample, the presence of a rhabdovirus could not be confirmed; surprisingly a tenuivirus was detected (melon chlorotic spot virus; MeCSV) ((Gaafar et al., 2019e), Chapter 2). The observed ribonucleoproteins (RNP) by EM in the original black medic sample were confused with the disassembled particles of rhabdoviruses. Nevertheless, these RNPs were the virions of the tenuivirus which are later confirmed in partially- purified virion preparations from infected plants.

The limitation of the conventional methods was further demonstrated with a beetroot sample with leaf necrosis, reduced size and root bearding: Using ISEM with various antibodies for the detection of various beetroot and sugar beet viruses, no virus could be detected ((Gaafar et al., 2019b), Chapter 2). Using HTS however, the full-length sequences of beet soil-borne virus and beet cryptic virus 2 could be recovered although BSBV specific-antibodies were also used in the ISEM. A similar example is represented by a celery sample where no virus particles could be observed with EM, and ELISA tests with antibodies against carrot red leaf virus and CLRV were negative ((Gaafar \& Ziebell, 2019a), Chapter 2). Nevertheless, we could detect a divergent strain of carrot torradovirus 1 (CaTV1) using HTS.

Furthermore, using MinION sequencer allowed us to rapidly identify the virus infecting several tomato samples i.e., Southern tomato virus ((Gaafar et al., 2019a), Chapter 2). Using dsRNA enrichment approach followed by MinION sequencing saved time allowing us to identify the virus in less than 24 hours. The speed at which this method was able to produce reads suggesting that it could be used in the future to as a laboratory diagnostic tool with a room for improvement in the quality of the produced raw reads and the costs of the platform and the sequencing kits.

\subsubsection{Pea survey in Germany using HTS}

In our survey on pea fields in Germany, HTS was used to detect viruses in peas and surrounding plants. In this survey, the plants were sampled from six different regions for three successive growing seasons. The samples were pooled together in ten pools i.e., 
region 1 to 6, and all symptomatic peas (SP), asymptomatic peas (aSP), surrounding legumes $(s L)$ and surrounding non-legumes $(s n L)$ for each season. Thirty-five viruses could be detected in addition to associated satellite nucleic acids and each finding was confirmed by RT-PCR. In addition to eleven known pea and legume viruses, nine new viruses and thirteen viruses new to Germany were reported (Chapter 3). Interestingly, new emaravirus provisionally called pea associated emaravirus, with close relationship to rose rosette virus, a quarantine pathogen, was detected in symptomatic peas in two seasons. The pooling strategy minimised the number of samples for HTS, nevertheless could not assist in identifying the exact host sample for each virus. Due to low virus titre or biased amplification of other nucleic acids, not all complete genomes of the viruses could be recovered demonstrating some limitations of the HTS-based study. Nevertheless, the discovery of nine novel viruses on one crop and its surrounding weeds imposes new challenges for diagnosticians, risk assessors and policy makers. Specific detection tests would need to be developed and implemented in future pea surveys to assess the presence of these viruses. As required by law, for each newly described virus a pest risk analysis would have to be carried out and policy makers would need to decide how to deal with these new viruses with respect to quarantine regulations and import/export restrictions.

\subsubsection{Virus characterisation}

Each newly identified virus was characterised molecularly (Chapter 2, 3 and 4). This was necessary for taxonomic assignment of these viruses and to characterise their potential open reading frames (ORF), motifs and domains in the viral sequences. For example, the isolates of pea enation mosaic virus 2 (PEMV2) from France and Germany have slightly shorter ORF3 (encoding a putative long-distance movement protein) than the PEMV2 reference sequence from the USA. This is due to a nucleotide substitution " $A$ to $T^{\prime \prime}$ at nt position 2783 in the French isolate and at nt position 2755 in German isolates. The first ORF (encoding a putative RNA-dependent RNA polymerase) of TuYVaRNA has an amber codon similar to other poleroviruses-associated RNAs which was not detected before in the available TuYVaRNA sequences on NCBI. Another example, is the potential new protease cleavage site "HS" that was identified in both predicted polyproteins of CawYV ((Gaafar et al., 2019f), Chapter 2).

These variations can contribute to the fitness and virulence of each virus. Such characteristics can be beneficial, neutral, deleterious or even lethal to the virus (Sanjuán, 2010). However, very little is known about the fitness and virulence costs of these characteristics. Therefore, it is important to confirm these molecular characteristics experimentally and to study their effects on virus infectivity, symptomology and fitness.

Moreover, EM helped us to study the virus particles morphology and with thin sections we could study the virion locations in infected cells. We could also confirm the relatedness of the novel viruses to their respective genera or family e.g., the presence of 
the replication factories in the nucleus of cells infected with AaNV (a feature of nucleorhabdoviruses) and the presence of tubular structure containing virus particles in CawYV infected plants (a feature of nepoviruses).

\subsubsection{Species demarcations set by ICTV}

Many new viruses were discovered in recent years using HTS technology. An advantage of these discoveries is the improvement of virus taxonomy. For example, in the Secoviridae family, all the members of the different genera are following the same "suggested" demarcations i.e., the conserved protease-polymerase (Pro-Pol) and the capsid protein (CP) regions share aa sequence identity $<80 \%$ and $<75 \%$ respectively, differences in antigenic reactions, distinct host range and vector specificity, absence of cross-protection and absence of re-assortment between RNA-1 and RNA-2 (for bipartite genome). Sanfaçon and colleagues suggested that these criteria need to be improved with more viral sequences of this family (Sanfaçon et al., 2009).

These criteria are challenging as not all of them are met at the same time. The novel CawYV ((Gaafar et al., 2019f); Chapter 2) had a Pro-Pol region which shared $80.1 \%$ amino acid (aa) identity to the closest virus, i.e., the value is $0.1 \%$ above the threshold set for Secoviridae species criteria therefore suggesting the presence of a novel strain of an existing species. In contrast, the CP region shared only $39.6 \%$ aa identity to its closest virus therefore this isolate should have been considered as new species. Other researchers face similar challenges, e.g., the discovery of red clover nepovirus A (RCNVA) (Koloniuk et al., 2018). The Pro-Pol region of RCNVA was $86 \%$ aa identical to artichoke Italian latent virus (AILV) and tomato black ring virus whereas the CP region was $64.4 \%$ aa to AILV. Moreover, the conserved regions i.e., the Pro-Pol and the CP regions of CLRV from carrot and CaTV1 (a torradovirus) from celery, both were detected again in the pea virome survey, also did not meet the Secoviridae species demarcations. However, they are divergent from their closest sequences in the other regions of the genomes e.g., genome length, whole ORFs and untranslated regions sequence identities (Chapter 2). This was also the same for other viruses e.g., tomato chocolate virus (torradovirus) (Verbeek et al., 2010).

We suggest that the ICTV Secoviridae study group revises the species demarcation criteria of Torradovirus and Nepovirus genera. We suggested not only reconsider the conserved regions but adding other criteria e.g., the identity of the nucleotide sequences of the untranslated regions and the amino acid identity of the whole predicted products of the ORFs. Verbeek and colleagues also suggested newly proposed additional demarcation criteria for the genus Torradovirus (Verbeek et al., 2010).

\subsubsection{Which viral enrichment approach is the best?}

Building on the success of HTS for plant virus and viroid detection, it is time to apply HTS for routine detection in laboratories. As with other new methods, HTS as a virus/viroid detection tool has some challenges. Firstly, due to their different genomic 
characteristics, there is no universal extraction method to fit all viruses/viroids. Secondly, there are no universal sequence for all viruses/viroids that can be amplified and used for sequencing such as the internal transcribed spacer (ITS) or 16S ribosomal RNA can be used for general detection of pathogens i.e., fungi and bacteria (Leff et al., 2017).Thirdly, viral enrichment is required during the sample preparation for HTS as the viral reads can be relatively very low compared to the host sequences (Adams \& Fox, 2016).

There are different enrichment methods are being used for virus/viroid detection by HTS. Each can differ in its efficiency and can have specific strengths and weaknesses (Adams \& Fox, 2016). In the performed studies (Chapter 2 and 3), different viral enrichment approaches have been used i.e., dsRNA extraction, ribosomal RNA depleted total RNA (ribo-depleted totRNA) extraction, small RNA extraction and rolling circle amplification (RCA). DsRNA extraction, ribo-depleted totRNA and small RNA (sRNA) viral enrichment approaches seem to be the most generic approaches recovering a range of plant viruses/viroids with different genome types and could be integrated in workflows in diagnostic laboratories.

A comparison between the three approaches was performed (Chapter 4). All known viruses were detected using the three viral enrichment methods. Surprisingly, two unknown viruses were detected by the dsRNA approach i.e., Vicia cryptic virus (VCV) and Wuhan aphid virus 2 (WHAV2), whereas only one of the two viruses was detected by the ribo-depleted totRNA and SRNA enrichment approaches, WHAV2 and VCV, respectively. The number of recovered viral reads were different from one method to another and were also virus dependent. The dsRNA approach used in this study detected all viruses (knowns and unknowns). It was cheaper, faster and easier compared to the other two approaches. Moreover, in comparison with other available dsRNA extraction methods, the dsRNA extraction kit used in this study is also faster, does not require a large amount of plant starting material and does not require an extra amplification step as in (Kesanakurti et al., 2016; Yanagisawa et al., 2016; Blouin et al., 2016).

The main limitation of this approach is the low concentration of extracted dsRNA which can be challenging for some library preparations. It is unclear whether PNYDV sequences (a DNA virus) were recovered as RNA/DNA hybrid molecule and amplified by HTS or if contaminating DNA was carried over in the dsRNA preparations (Knierim et al., 2019).

\subsubsection{Data analysis}

There are different software and tools used for bioinformatic analyses e.g., HTS data analysis and molecular characterisation of the findings (Bao et al., 2014). The algorithms used for analysis are different from one bioinformatic tool to another (Miller et al., 2010; Li \& Homer, 2010; Narzisi \& Mishra, 2011). Changing the parameters used in the analyses can be beneficial where unknown findings can be detected or can be incorrect thus some data might be concealed (Del Fabbro et al., 2013; Massart et al., 
2019). For virus/viroid detection, there are different thresholds set by each laboratory and there are no generic criteria set for all. Some laboratories a very strict with the number of reads and coverage to consider a finding and thus confirm it. Such restrictions can cause the ignorance of some findings e.g., viruses with low titre. We confirmed all the findings even with low number of reads and coverage. This led to the identification and confirmation of many viruses (Chapter 2, 3 and 4).

The computer facility was a limitation with large data and with some tools i.e., the analysis of sRNA data required a strong hardware computer with larger memory and stronger processor. Thus, we tend to normalise the reads and remove duplicated reads. This facilitated the subsequent analyses e.g., de novo assembly on such low hardware specifications computer.

Additionally, in some of our analysed samples no viruses or viroids were detected. As our focus is plant viruses and viroids, our Basic Local Alignment Search Tool (BLAST) databases do not contain sequences of other pathogens e.g., phytoplasma or fungi.

\subsection{Part 2: Virus-vector-host interactions}

\subsubsection{Nanoviruses infection change the probing and feeding behaviours of aphids}

To investigate the effect of nanoviruses infection on the probing and feeding behaviours of pea aphids (Acyrthosiphon pisum) on faba beans (Vicia faba), electrical penetration graph (EPG) was used (Chapter 6). Our results showed that infections of $V$. faba plants with nanoviruses i.e., faba bean necrotic yellows virus (FBNYV) and PNYDV changed the feeding and probing behaviours of $A$. pisum where the aphids spent less probing time on infected plants compared to those on healthy plants. Furthermore, aphids spent less time phloem feeding compared to those on healthy plants. The FBNYVinfected plants displayed severe disease symptoms compared to PNYDV-infected plants and the changes in the behaviours of the aphids were higher on FBNYV-infected than those on PNYDV-infected. It is possible that these behavioural changes are correlated to the disease development (changes in the chemical composition and physical structure of infected plant tissue).

\subsubsection{Absence of the nuclear shuttle protein does not affect the aphid probing and feeding behaviours}

The nuclear shuttle protein (NSP) is encoded by DNA-N of nanoviruses and is required for the aphid transmission (Grigoras et al., 2018). To investigate the effect of NSP on the probing and feeding behaviours of the aphids, plants were agroinoculated with infectious clones of all eight components of faba bean necrotic stunt virus (FBNSV) i.e., DNA-C, - M, -N, -R, -S, -U1, -U2 and -U4. In addition, other plants were agroinoculated with seven components omitting DNA-N (FBNSV/-N). Two additional controls were used i.e., plants inoculated with agrobacteria without the infectious clones and healthy plants without agroinoculation. No significant changes in feeding behaviour could be observed 
when A. pisum fed on FBNSV/-N-infected plants compared to those fed on FBNSV-infected plants (Chapter 6). This can be due to that nanoviruses symptoms can be developed without DNA-N (Timchenko et al., 2006). The only significant difference from the healthy control was derailed stylet mechanics (F) which was higher on FBNSV/-N infected plants. We also found significant effects for the agrobacteria on the behaviours where $F$ was higher than on healthy plants "without agroinoculation". The intercellular structural composition is suggested to be responsible for these derailments (Tjallingii, 1988). The increase in derailing of the stylet with the presence of agrobacteria was reduced in case of FBNSV-infected plants. This suggests that the agrobacteria may have altered the effect of the virus.

\subsection{General conclusion and future perspectives}

I have demonstrated the strength of HTS as a rapid and accurate diagnostics tool for plant virus discovery, characterisation and for metagenomics studies. During the optimisation process I found out that viral enrichment based on dsRNA approach were better than the ribo-depleted totRNA and SRNA approaches for HTS virus/viroid detection. It is important to follow-up with a comparison of different dsRNA enrichment approaches to reach the most reliable viral enrichment approach. This will help fastening the application of HTS as a diagnostic tool in laboratories. Further research is needed to identify the natural vectors and mode of transmission of the new viruses as well as their host range. Moreover, it is important to test experimentally the molecular characteristics of the new viruses and their effect on virulence and fitness.

Aphid-borne nanoviruses are threatening legume production in different countries. Infection of nanoviruses of $V$. faba caused reduction in $A$. pisum's probing and feeding behaviours which may be related to changes in the chemical composition and physical structure of plant tissues during disease development. Consequently, the aphids may not develop colonies on the infected plants and will spread wider within the field. It is recommended testing the dynamic of changes in these behaviours with the disease development and to relate them to the virus titres and to compare the behaviours on symptomatic and asymptomatic leaves. Furthermore, the absence of the NSP did not affect the feeding behaviour of $A$. pisum, however there was an effect of agroinoculation observed. Thus, we recommend experimenting with additional controls i.e., FBNSVinfected plants after successive aphid transmission to eliminate or reduce the effect of agrobacteria, plants inoculated with inoculation buffer without bacteria and plants with

needle injection only. 


\section{Summary}

The overall aim of this dissertation was the implementation of high-throughput sequencing (HTS) technologies for plant viruses' identification and characterisation. Different viral enrichment approaches were tested to optimise a tool that would detect a broad range of different viruses and that can be used as a generic, reliable and costeffective strategy for virus diagnostics. Moreover, the interactions of aphid vectors of nanoviruses with infected plants were investigated and the effect of NSP on aphid feeding behaviour were closely observed.

Chapter 1 gives a general introduction to the concept of this research, and it also outlines the aims of this work. Currently used plant virus diagnostics methods are described with a focus on HTS for virus detection using different enrichment and platforms. It also covers the virus-vector-host interactions with focus on the model system nanoviruses-aphid-host used in this research.

In addition, this thesis is divided into two parts (part one and part two). Part one focuses on the plant virus identification and describes the discovery and characterisation of new viruses, divergent strains and isolates from new virus hosts or geographical locations. The virome of peas was investigated over a period of three growing seasons in six different German regions. Furthermore, three viral enrichment approaches for plant viruses and viroids detection by HTS were compared. Part one contains three chapters (2, 3 and 4). In Chapter 2, the discovery and characterisation of two new viruses i.e., alfalfa associated nucleorhabdovirus from Medicago sativa (Austria) and caraway yellows virus from Carum carvi (Germany)are described.

Moreover, the reports on the identification and characterisation of the causal agent of several diseases in legumes and vegetables. HTS in combination with conventional diagnostic methods (ELISA, EM, PCR-based methods and Sanger sequencing) were applied to various plant virus samples in the JKI selection. The following viruses were described:

- Divergent strains of:

1. Melon chlorotic spot virus from M. Iupulina from Austria

2. Carrot torradovirus 1 from Apium graveolens from Germany

3. Turnip crinkle virus from Alliaria petiolata from Germany

- Divergent isolates of turnip yellows virus (TuYV) from pea and rapeseed oil, in addition to a first report of turnip yellows associated RNA from Germany

- First reports of:

1. Beet soil borne virus and beet cryptic virus 2 from Beta vulgaris subsp. vulgaris

2. Physostegia chlorotic mottle virus from Solanum lycopersicum from Germany

3. Southern tomato virus from S. lycopersicum from Germany (new MinION approach was described) 
4. Pea necrotic yellow dwarf virus (PNYDV) was detected for the first time infecting Vicia faba, V. sativa and Lens culinaris in Austria and Germany, and for the first time in Denmark and the Netherlands.

The focus in chapter $\mathbf{3}$ is set on the identification and characterisation of viruses in German pea fields. For three successive seasons, pea fields in six different regions were surveyed. Samples were taken from symptomatic pea and asymptomatic pea plants, in addition, samples from surrounding non-crop legume and non-legumes weeds were taken. The samples were analysed using HTS and the presence of all detected viruses was confirmed by RT-PCR. Thirty-five viruses in total were detected during this survey, including nine new viruses, thirteen viruses new for Germany. In addition to plant viruses, virus associated nucleic acids were also detected. Pea enation mosaic virus 1 and 2, TuYV, and PNYDV were the most common viruses in the German pea fields. Interestingly a new emaravirus was detected for two successive seasons in the same region, tentatively called pea associated emaravirus. Additionally, several new virus sequences and divergent sequences were detected in the surrounding legumes and non-legumes. By analysing the data spatially and temporally, no differences were observed.

Chapter 4 describes the comparison of three different viral enrichment approaches for virus discovery by HTS. Double stranded (dsRNA), ribosomal RNA depleted total RNA (ribo-depleted totRNA) and small RNA (sRNA) enrichment approaches were compared for the detection of viruses and a viroid representing different genomes i.e., ssRNA [(+ve) and (-ve) senses], DNA and a viroid. The dsRNA approach in this study was better compared to the other two approaches as it is faster, cheaper and all the known and unknown viruses/viroid in the study were detected. However, the number of reads from dsRNA approach were lower when compared to the other two methods. Interestingly, two additional unknown viruses were discovered with the dsRNA enrichment approach i.e., Vicia cryptic virus and Wuhan aphid virus 2 (WHAV2) but only one of them was discovered with SRNA or ribo-depleted totRNA, respectively. To our knowledge this is the first detection of WHAV2, a recently discovered virus from aphids in China, from plant tissues.

In the second part of this thesis (part two), the interactions of nanoviruses with their insect vectors and their hosts were studied. Chapter 5 reviews the current knowledge about nanovirus-vector interactions. In chapter 6, the probing and feeding behaviours of Acyrthosiphon pisum on nanoviruses-infected faba beans were investigated using electrical penetration graph. The probing and feeding behaviours of $A$. pisum changed on faba bean necrotic yellows virus and PNYDV-infected plants. Additionally, the effect of the absence of the nuclear shuttle protein of FBNSV on the feeding and probing behaviours of $A$. pisum was tested. Additionally, few significant differences were detected, however no clear differences could be concluded. 
A general discussion of the findings of this thesis is laid out in the final chapter (7), and a prospect for future research is given. 


\section{References}

Abbas Q, Amin I, Mansoor S, 2019. The Rep proteins encoded by alphasatellites restore expression of a transcriptionally silenced green fluorescent protein transgene in Nicotiana benthamiana. Virus Disease 30, 101-5. doi: 10.1007/s13337-017-0413-5.

Abraham AD, Menzel W, Lesemann D-E, 2006. Chickpea chlorotic stunt virus: a new polerovirus infecting cool-season food legumes in Ethiopia. Phytopathology 96, 437-46. doi: 10.1094/PHYTO-96-0437.

Abraham AD, Menzel W, Vetten HJ, 2007. First report of Soybean dwarf virus (genus Luteovirus) infecting faba bean and clover in Germany. Plant Disease 91, 1059. doi: 10.1094/PDIS-91-81059B.

Abraham AD, Varrelmann M, Josef Vetten $H, 2012$. Three distinct nanoviruses, one of which represents a new species, infect faba bean in Ethiopia. Plant Disease 96, 1045-53. doi: 10.1094/PDIS-09-11-0734-RE.

Adams I, Fox A, 2016. Diagnosis of plant viruses using next-generation sequencing and metagenomic analysis. In: Wang Z, ed. Current Research Topics in Plant. Vol 10, 323-35.

Adams IP, Glover RH, Monger WA et al., 2009. Next-generation sequencing and metagenomic analysis: a universal diagnostic tool in plant virology. Molecular Plant Pathology 10, 537-45. doi: 10.1111/j.1364-3703.2009.00545.x.

Adams IP, Skelton A, Macarthur R et al., 2014. Carrot yellow leaf virus is associated with carrot internal necrosis. PLOS ONE 9, e109125. doi: 10.1371/journal.pone.0109125.

Adams MJ, Adkins S, Bragard C et al., 2017. ICTV virus taxonomy profile: Virgaviridae. The Journal of General Virology 98, 1999-2000. doi: 10.1099/jgv.0.000884.

Adasme-Carreño F, Muñoz-Gutiérrez C, Salinas-Cornejo J, 2015. A2EPG. A new software for the analysis of electrical penetration graphs to study plant probing behaviour of hemipteran insects. Computers and Electronics in Agriculture 113, 128-35. doi:

10.1016/j.compag.2015.02.005.

Al Rwahnih M, Daubert S, Golino D, 2009. Deep sequencing analysis of RNAs from a grapevine showing Syrah decline symptoms reveals a multiple virus infection that includes a novel virus. Virology 387, 395-401. doi: 10.1016/j.virol.2009.02.028.

Alliot B, Signoret PA, 1972. La «maladie àénations de la Luzerne», une maladie nouvelle pour la France. Journal of Phytopathology 74, 69-73. doi: 10.1111/j.1439-0434.1972.tb04647.x.

Alvarez AE, Garzo E, Verbeek M, 2007. Infection of potato plants with potato leafroll virus changes attraction and feeding behaviour of Myzus persicae. Entomologia Experimentalis et Applicata 125, 135-44. doi: 10.1111/j.1570-7458.2007.00607.x.

Amarasinghe GK, Aréchiga Ceballos NG, Banyard AC et al., 2018. Taxonomy of the order Mononegavirales: update 2018. Archives of Virology 163, 2283-94. doi: 10.1007/s00705-0183814-x. 
Anderson PK, Cunningham AA, Patel NG, 2004. Emerging infectious diseases of plants: pathogen pollution, climate change and agrotechnology drivers. Trends in Ecology \& Evolution 19, 53544. doi: 10.1016/j.tree.2004.07.021.

Angelella G, Nalam V, Nachappa P, 2018. Endosymbionts differentially alter exploratory probing behavior of a nonpersistent plant virus vector. Microbial Ecology 76, 453-8. doi: 10.1007/s00248-017-1133-5.

Antoniw JF, White RF, Xie W, 1990. Cryptic viruses of beet and other plants. In: Fraser RSS, ed. Recognition and response in plant-virus interactions, Springer: Berlin, Heidelberg, 273-86.

Argos P, Kamer G, Nicklin MJ, 1984. Similarity in gene organization and homology between proteins of animal picornaviruses and a plant comovirus suggest common ancestry of these virus families. Nucleic Acids Research 12, 7251-67.

Augusto Lopez R, Percy Miranda P, Edgar Tejada V, 1992. Outbreak of human rabies in the Peruvian jungle. The Lancet 339, 408-11. doi: 10.1016/0140-6736(92)90088-K.

Axén C, Hakhverdyan M, Boutrup TS et al., 2017. Emergence of a new rhabdovirus associated with mass mortalities in eelpout (Zoarces viviparous) in the Baltic Sea. Journal of Fish Diseases 40, 219-29. doi: 10.1111/jfd.12506.

Bailer J, Aichinger T, Hackl G, 2001. Essential oil content and composition in commercially available dill cultivars in comparison to caraway. Industrial Crops and Products 14, 229-39. doi: 10.1016/S0926-6690(01)00088-7.

Bankevich A, Nurk S, Antipov D et al., 2012. SPAdes: a new genome assembly algorithm and its applications to single-cell sequencing. Journal of Computational Biology 19, 455-77. doi: $10.1089 / \mathrm{cmb} .2012 .0021$.

Bao R, Huang L, Andrade J et al., 2014. Review of current methods, applications, and data management for the bioinformatics analysis of whole exome sequencing. Cancer Informatics 13, 67-82. doi: 10.4137/CIN.S13779.

Bardin SD, Huang HC, 2001. Research on biology and control of Sclerotinia diseases in Canada 1. Canadian Journal of Plant Pathology 23, 88-98. doi: 10.1080/07060660109506914.

Bar-Joseph M, Rosner A, Moscovitz M, 1983. A simple procedure for the extraction of doublestranded RNA from virus-infected plants. Journal of Virological Methods 6, 1-8. doi: 10.1016/0166-0934(83)90062-9.

Barnett OW, 1987. Relationships among Australian and North American isolates of the bean yellow mosaic potyvirus subgroup. Phytopathology 77, 791. doi: 10.1094/Phyto-77-791.

Baumann P, Baumann L, Lai CY, 1995. Genetics, physiology, and evolutionary relationships of the genus Buchnera: intracellular symbionts of aphids. Annual Review of Microbiology 49, 55-94. doi: 10.1146/annurev.mi.49.100195.000415.

Bawden FC, Nixon HL, 1951. The application of electron microscopy to the study of plant viruses in unpurified plant extracts. Journal of General Microbiology 5, 104-9. doi: 10.1099/00221287-5-1-104.

Behr H-C, 2015. AMI Markt Bilanz Gemüse 2015. Bonn, Germany: Agrarmarkt InformationsGesellschaft mbH. 
Behr H-C, 2019. AMI Markt Bilanz Gemüse 2019. Bonn, Germany: Agrarmarkt InformationsGesellschaft mbH.

Bejerman N, Giolitti F, Breuil S de et al., 2015. Complete genome sequence and integrated protein localization and interaction map for alfalfa dwarf virus, which combines properties of both cytoplasmic and nuclear plant rhabdoviruses. Virology 483, 275-83. doi: 10.1016/j.virol.2015.05.001.

Bejerman N, Nome C, Giolitti F et al., 2011. First report of a rhabdovirus infecting alfalfa in Argentina. Plant Disease 95, 771. doi: 10.1094/PDIS-10-10-0764.

Bem F, Murant AF, 1979. Host range, purification and serological properties of heracleum latent virus. Annals of Applied Biology 92, 243-56. doi: 10.1111/j.1744-7348.1979.tb03870.x.

Bergeson GB, Athow KL, Laviolette FA, 1964. Transmission, movement, and vector relationships of tobacco ringspot virus in soybean. Phytopathology 54, 723-8.

Bernad L, Duran-Vila N, 2006. A novel RT-PCR approach for detection and characterization of citrus viroids. Molecular and Cellular Probes 20, 105-13. doi: 10.1016/j.mcp.2005.11.001.

Bioreba, 2014. ELISA data analysis. [http://www.bioreba.ch/saas/CustomUpload/37403570340037003560369035003210360 O36603690356035303520350032003260/ELISA_Data_Analysis.pdf]. Accessed 25 October 2018.

Blanc S, Drucker M, Uzest M, 2014. Localizing viruses in their insect vectors. Annual Review of Phytopathology 52, 403-25. doi: 10.1146/annurev-phyto-102313-045920.

Blawid R, Stephan D, Maiss E, 2007. Molecular characterization and detection of Vicia cryptic virus in different Vicia faba cultivars. Archives of Virology 152, 1477-88. doi: 10.1007/s00705007-0966-5.

Blouin AG, Pearson MN, Chavan RR et al., 2013. Viruses of kiwifruit (Actinidia species). Journal of Plant Pathology, 221-35.

Blouin AG, Ross HA, Hobson-Peters J, 2016. A new virus discovered by immunocapture of double-stranded RNA, a rapid method for virus enrichment in metagenomic studies.

Molecular Ecology Resources 16, 1255-63. doi: 10.1111/1755-0998.12525.

BMEL, 2019. Eiweißpflanzenstrategie.

[https://www.bmel.de/DE/Landwirtschaft/Pflanzenbau/Ackerbau/_Texte/Eiweisspflanzenstra tegie.html]. Accessed 4 July 2019.

Bolton MD, Thomma BPHJ, Nelson BD, 2006. Sclerotinia sclerotiorum (Lib.) de Bary: biology and molecular traits of a cosmopolitan pathogen. Molecular Plant Pathology 7, 1-16. doi: 10.1111/j.1364-3703.2005.00316.x.

Boonham N, Kreuze J, Winter S et al., 2014. Methods in virus diagnostics: from ELISA to next generation sequencing. Virus Research 186, 20-31. doi: 10.1016/j.virusres.2013.12.007.

Boquel S, Giordanengo P, Ameline A, 2011. Divergent effects of PVY-infected potato plant on aphids. European Journal of Plant Pathology 129, 507-10. doi: 10.1007/s10658-010-9732-8. 
Bos L, 1970. The identification of three new viruses isolated from Wisteria and Pisum in The Netherlands, and the problem of variation within the potato virus $Y$ group. Netherlands Journal of Plant Pathology 76, 8-46. doi: 10.1007/BF01976763.

Bos L, 1982. Crop losses caused by viruses. Crop Protection 1, 263-82. doi: 10.1016/02612194(82)90002-3.

Bos L, Delević B, Want JPH, 1959. Investigations on white clover mosaic virus. Netherlands Journal of Plant Pathology 65, 89-106. doi: 10.1007/BF02112372.

Bos L, Hampton RO, Makkouk KM, 1988. Viruses and virus diseases of pea, lentil, faba bean and chickpea. In: Summerfield RJ, ed. World crops: Cool season food legumes. A global perspective of the problems and prospects for crop improvement in pea, lentil, faba bean and chickpea, Springer Netherlands: Dordrecht. Vol 5, 591-615.

Brault $\mathrm{V}$, Herrbach E, Reinbold C, 2007. Electron microscopy studies on luteovirid transmission by aphids. Micron 38, 302-12. doi: 10.1016/j.micron.2006.04.005.

Brault V, Mutterer J, Scheidecker D et al., 2000. Effects of point mutations in the readthrough domain of the beet western yellows virus minor capsid protein on virus accumulation in planta and on transmission by aphids. Journal of Virology 74, 1140-8. doi: 10.1128/jvi.74.3.1140-1148.2000.

Brault V, Périgon S, Reinbold C et al., 2005. The polerovirus minor capsid protein determines vector specificity and intestinal tropism in the aphid. Journal of Virology 79, 9685-93. doi: 10.1128/JVI.79.15.9685-9693.2005.

Brault V, Tanguy S, Reinbold C et al., 2010. Transcriptomic analysis of intestinal genes following acquisition of pea enation mosaic virus by the pea aphid Acyrthosiphon pisum. The Journal of General Virology 91, 802-8. doi: 10.1099/vir.0.012856-0.

Brault V, van den Heuvel JF, Verbeek $\mathrm{M}$ et al., 1995. Aphid transmission of beet western yellows luteovirus requires the minor capsid read-through protein P74. The EMBO Journ/ 14, 650-9.

Bressan A, Watanabe S, 2011. Immunofluorescence localisation of Banana bunchy top virus (family Nanoviridae) within the aphid vector, Pentalonia nigronervosa, suggests a virus tropism distinct from aphid-transmitted luteoviruses. Virus Research 155, 520-5. doi: 10.1016/j.virusres.2010.12.005.

Briddon RW, Ghabrial S, Lin NS, 2012. Satellites and other virus-dependent nucleic acids. In: King AMQ, Lefkowitz E, Adams MJ, Carstens EB, eds. Virus taxonomy: ninth report of the International Committee on Taxonomy of Viruses, Elsevier academic press: Oxford, 1211-9.

Briddon RW, Martin DP, Roumagnac P et al., 2018. Alphasatellitidae: a new family with two subfamilies for the classification of geminivirus- and nanovirus-associated alphasatellites. Archives of Virology 163, 2587-600. doi: 10.1007/s00705-018-3854-2.

Broadbent L, Heathcote DG, 1958. Properties and host range of turnip crinkle, rosette and yellow mosaic viruses. Annals of Applied Biology 46, 585-92. doi: 10.1111/j.17447348.1958.tb02242.x.

Bruenn JA, Warner BE, Yerramsetty P, 2015. Widespread mitovirus sequences in plant genomes. PeerJ 3, e876. doi: 10.7717/peerj.876. 
Brunissen L, Cherqui A, Pelletier Y, 2009. Host-plant mediated interactions between two aphid species. Entomologia Experimentalis et Applicata 132, 30-8. doi: 10.1111/j.1570-

7458.2009.00862.x.

Bruyère $A$, Brault $V$, Ziegler-Graff $V$ et al., 1997. Effects of mutations in the beet western yellows virus readthrough protein on its expression and packaging and on virus accumulation, symptoms, and aphid transmission. Virology 230, 323-34. doi: 10.1006/viro.1997.8476.

Buchner P, 1965. Endosymbiosis of animals with plant microorganisms. New York: Interscience Publishers.

Büttner C, Bargen S von, Bandte M, 2011. Cherry leaf roll virus. In: Hadidi A, Barba M, Candresse $\mathrm{T}$, Jelkmann W, eds. Virus and virus-like diseases of pome and stone fruits, APS Press/American Phytopathological Society: St. Paul, Minnesota, 119-25.

Camacho C, Coulouris G, Avagyan V et al., 2009. BLAST+: architecture and applications. BMC Bioinformatics 10, 421. doi: 10.1186/1471-2105-10-421.

Candresse T, Marais A, Faure C, 2013. First report of Southern tomato virus on tomatoes in Southwest France. Plant Disease 97, 1124. doi: 10.1094/PDIS-01-13-0017-PDN.

Canto T, Aranda MA, Fereres A, 2009. Climate change effects on physiology and population processes of hosts and vectors that influence the spread of hemipteran-borne plant viruses. Global Change Biology 15, 1884-94.

Cardin L, Delecolle B, Moury B, 2009. First report of cucumber mosaic virus and turnip veinclearing virus in Dichondra repens in France, Italy, and China. Plant Disease 93, 201. doi: 10.1094/PDIS-93-2-0201B.

Carvajal-Yepes M, Olaya C, Lozano I, 2014. Unraveling complex viral infections in cassava (Manihot esculenta Crantz) from Colombia. Virus Research 186, 76-86. doi: 10.1016/j.virusres.2013.12.011.

Carvalho CM, Santos AA, Pires SR et al., 2008. Regulated nuclear trafficking of rpL10A mediated by NIK1 represents a defense strategy of plant cells against virus. PLOS Pathogens 4 , e1000247. doi: 10.1371/journal.ppat.1000247.

Casper R, Meyer S, 1981. Anwendung des ELISA-Verfahrens zum Nachweis pflanzenpathogener Viren. Nachrichtenblatt des Deutschen Pflanzenschutzdienstes 33, 49-54.

Castle SJ, Berger PH, 1993. Rates of growth and increase of Myzus persicae on virus-infected potatoes according to type of virus-vector relationship. Entomologia Experimentalis et Applicata 69, 51-60. doi: 10.1111/j.1570-7458.1993.tb01727.x.

Castle SJ, Mowry TM, Berger PH, 1998. Differential settling by Myzus persicae (Homoptera: Aphididae) on various virus infected host plants. Annals of the Entomological Society of America 91, 661-7. doi: 10.1093/aesa/91.5.661.

Chatzivassiliou EK, Giakountis A, Kumari SG, 2016. Viruses affecting lentil (Lens culinaris Medik.) in Greece; incidence and genetic variability of Bean leafroll virus and Pea enation mosaic virus. Phytopathologia Mediterranea 55, 239-52. 
Chavez JD, Cilia M, Weisbrod CR et al., 2012. Cross-linking measurements of the Potato leafroll virus reveal protein interaction topologies required for virion stability, aphid transmission, and virus-plant interactions. Journal of Proteome Research 11, 2968-81. doi: 10.1021/pr300041t.

Chen H, Boutros PC, 2011. VennDiagram: a package for the generation of highly-customizable Venn and Euler diagrams in R. BMC Bioinformatics 12, 35. doi: 10.1186/1471-2105-12-35.

Chen S, Jiang G, Wu J, 2016. Characterization of a novel polerovirus infecting maize in China. Viruses 8. doi: 10.3390/v8050120.

Chin LS, Foster JL, Falk BW, 1993. The beet western yellows virus ST9-associated RNA shares structural and nucleotide sequence homology with carmo-like viruses. Virology 192, 473-82. doi: 10.1006/viro.1993.1063.

Chu PW, Helms K, 1988. Novel virus-like particles containing circular single-stranded DNAs associated with subterranean clover stunt disease. Virology 167, 38-49.

Cilia M, Peter KA, Bereman MS et al., 2012. Discovery and targeted LC-MS/MS of purified polerovirus reveals differences in the virus-host interactome associated with altered aphid transmission. PLOS ONE 7, e48177. doi: 10.1371/journal.pone.0048177.

Clark MF, Adams AN, 1977. Characteristics of the microplate method of enzyme-linked immunosorbent assay for the detection of plant viruses. The Journal of General Virology 34, 475-83. doi: 10.1099/0022-1317-34-3-475.

Clement SL, 2006. Pea aphid outbreaks and virus epidemics on peas in the US Pacific Northwest: histories, mysteries, and challenges. Plant Health Progress 7, 34. doi: 10.1094/PHP-20061018-01-RV.

Coetzee B, Freeborough M-J, Maree HJ, 2010. Deep sequencing analysis of viruses infecting grapevines: Virome of a vineyard. Virology 400, 157-63. doi: 10.1016/j.virol.2010.01.023.

Cohen D, Chavan RR, Blouin AG, 2012. First report of Turnip vein clearing virus and Ribgrass mosaic virus from New Zealand. Australasian Plant Disease Notes 7, 67-9. doi: 10.1007/s13314-012-0050-1.

Collar JL, Avilla C, Fereres A, 1997. New correlations between aphid stylet paths and nonpersistent virus transmission. Environmental Entomology 26, 537-44. doi: 10.1093/ee/26.3.537.

Cooper WR, Goggin FL, 2005. Effects of jasmonate-induced defenses in tomato on the potato aphid, Macrosiphum euphorbiae. Entomologia Experimentalis et Applicata 115, 107-15. doi: 10.1111/j.1570-7458.2005.00289.x.

Coutts RHA, Livieratos IC, 2003. A rapid method for sequencing the 5'- and 3'-termini of doublestranded RNA viral templates using RLM-RACE. Journal of Phytopathology 151, 525-7. doi: 10.1046/j.1439-0434.2003.00755.x.

Cropley R, 1961. Cherry leaf-roll virus. Annals of Applied Biology 49, 524-9. doi: 10.1111/j.17447348.1961.tb03645.x.

Czotter N, Molnar J, Szabó E et al., 2018. NGS of virus-derived small RNAs as a diagnostic method used to determine viromes of Hungarian vineyards. Frontiers in Microbiology 9, 758. doi: 10.3389/fmicb.2018.00122. 
Damsteegt VD, Hewings AD, 1986. Comparative transmission of soybean dwarf virus by three geographically diverse populations of Aulacorthum (= Acyrthosiphon) solani. Annals of Applied Biology 109, 453-63. doi: 10.1111/j.1744-7348.1986.tb03202.x.

Dean FB, Nelson JR, Giesler TL, 2001. Rapid amplification of plasmid and phage DNA using Phi 29 DNA polymerase and multiply-primed rolling circle amplification. Genome Research 11, 10959. doi: 10.1101/gr.180501.

Debrick KS, 1973. Quantitative assay for plant viruses using serologically specific electron microscopy. Virology 56, 652-3. doi: 10.1016/0042-6822(73)90068-8.

Del Fabbro C, Scalabrin S, Morgante M, 2013. An extensive evaluation of read trimming effects on Illumina NGS data analysis. PLOS ONE 8, e85024. doi: 10.1371/journal.pone.0085024.

Demler SA, Rucker DG, Nooruddin L, 1994. Replication of the satellite RNA of pea enation mosaic virus is controlled by RNA 2-encoded functions. Journal of General Virology 75 (Pt 6), 1399406. doi: 10.1099/0022-1317-75-6-1399.

Demler SA, Zoeten GA de, 1989. Characterization of a satellite RNA associated with pea enation mosaic virus. Journal of General Virology 70, 1075-84. doi: 10.1099/0022-1317-70-5-1075.

Dietzgen RG, Innes DJ, Bejerman N, 2015. Complete genome sequence and intracellular protein localization of datura yellow vein nucleorhabdovirus. Virus Research 205, 7-11. doi: 10.1016/j.virusres.2015.05.001.

Dilcher M, Faye O, Faye O et al., 2015. Zahedan rhabdovirus, a novel virus detected in ticks from Iran. Virology Journal 12, 183. doi: 10.1186/s12985-015-0410-5.

Ding S-W, 2010. RNA-based antiviral immunity. Nature Reviews Immunology 10, 632-44. doi: $10.1038 /$ nri2824.

Ding S-W, Lu R, 2011. Virus-derived siRNAs and piRNAs in immunity and pathogenesis. Current Opinion in Virology 1, 533-44. doi: 10.1016/j.coviro.2011.10.028.

Dodds JA, Morris TJ, Jordan RL, 1984. Plant viral double-stranded RNA. Annual Review of Phytopathology 22, 151-68. doi: 10.1146/annurev.py.22.090184.001055.

Dorokhov YL, Ivanov PA, Novikov VK et al., 1994. Complete nucleotide sequence and genome organization of a tobamovirus infecting cruciferae plants. FEBS Letters 350, 5-8. doi: 10.1016/0014-5793(94)00721-7.

Douglas GB, Wang Y, Waghorn GC et al., 1995. Liveweight gain and wool production of sheep grazing Lotus corniculatus and lucerne (Medicago sativa). New Zealand Journal of Agricultural Research 38, 95-104. doi: 10.1080/00288233.1995.9513108.

Doumayrou J, Sheber M, Bonning BC, 2017. Quantification of Pea enation mosaic virus 1 and 2 during infection of Pisum sativum by one step real-time RT-PCR. Journal of Virological Methods 240, 63-8. doi: 10.1016/j.jviromet.2016.11.013.

Drijfhout E, Bos L, 1977. The identification of two new strains of bean common mosaic virus. Netherlands Journal of Plant Pathology 83, 13-25. doi: 10.1007/BF01976508. 
Durand P, Canard L, Mornon JP, 1997. Visual BLAST and Visual FASTA: graphic workbenches for interactive analysis of full BLAST and FASTA outputs under Microsoft Windows 95/NT.

Bioinformatics 13, 407-13. doi: 10.1093/bioinformatics/13.4.407.

Eddouks M, Lemhadri A, Michel J-B, 2004. Caraway and caper: potential anti-hyperglycaemic plants in diabetic rats. Journal of Ethnopharmacology 94, 143-8. doi:

10.1016/j.jep.2004.05.006.

Edgar RC, 2004. MUSCLE: multiple sequence alignment with high accuracy and high throughput. Nucleic Acids Research 32, 1792-7. doi: 10.1093/nar/gkh340.

Edwards K, Johnstone C, Thompson C, 1991. A simple and rapid method for the preparation of plant genomic DNA for PCR analysis. Nucleic Acids Research 19, 1349.

Eid J, Fehr A, Gray J et al., 2009. Real-time DNA sequencing from single polymerase molecules. Science (New York, N.Y.) 323, 133-8. doi: 10.1126/science.1162986.

Eigenbrode SD, Ding H, Shiel P, 2002. Volatiles from potato plants infected with potato leafroll virus attract and arrest the virus vector, Myzus persicae (Homoptera: Aphididae). Proceedings: Biological Sciences 269, 455-60. doi: 10.1098/rspb.2001.1909.

Elbeaino T, Digiaro M, Mielke-Ehret N, 2018. ICTV Virus Taxonomy Profile: Fimoviridae. The Journal of General Virology 99, 1478-9. doi: 10.1099/jgv.0.001143.

Elbeaino T, Digiaro M, Uppala M, 2014. Deep sequencing of pigeonpea sterility mosaic virus discloses five RNA segments related to emaraviruses. Virus Research 188, 27-31. doi: 10.1016/j.virusres.2014.03.022.

Elbeaino T, Digiaro M, Uppala M, 2015. Deep sequencing of dsRNAs recovered from mosaicdiseased pigeonpea reveals the presence of a novel emaravirus: pigeonpea sterility mosaic virus 2. Archives of Virology 160, 2019-29. doi: 10.1007/s00705-015-2479-y.

EPPO, 2019. EPPO A1 List. [https://www.eppo.int/ACTIVITIES/plant_quarantine/A1_list]. Accessed 30 August 2019.

Falk BW, Tsai JH, 1998. Biology and molecular biology of viruses in the genus Tenuivirus. Annual Review of Phytopathology 36, 139-63. doi: 10.1146/annurev.phyto.36.1.139.

Fletcher J, Tang J, Blouin A, 2016. Red clover vein mosaic virus - a novel virus to New Zealand that is widespread in legumes. Plant Disease 100, 890-5. doi: 10.1094/PDIS-04-15-0465-RE.

Fletcher JD, 1993. Surveys of virus diseases in pea, lentil, dwarf and broad bean crops in South Island, New Zealand. New Zealand Journal of Crop and Horticultural Science 21, 45-52. doi: 10.1080/01140671.1993.9513745.

Fletcher JD, Goulden DS, Russell AC, 1989. Breeding for resistance to pea seed-borne mosaic virus. Grain Legumes: National Symposium and Workshop. Agronomy Society of New Zealand Special Publication 7, 124.

Flusberg BA, Webster DR, Lee JH et al., 2010. Direct detection of DNA methylation during singlemolecule, real-time sequencing. Nature Methods 7, 461-5. doi: 10.1038/nmeth.1459. 
Fontes EPB, Santos AA, Luz DF, 2004. The geminivirus nuclear shuttle protein is a virulence factor that suppresses transmembrane receptor kinase activity. Genes \& Development 18, 2545-56. doi: 10.1101/gad.1245904.

Foyer $\mathrm{CH}$, Lam H-M, Nguyen HT et al., 2016. Neglecting legumes has compromised human health and sustainable food production. Nature plants 2, 16112. doi: 10.1038/nplants.2016.112.

Frago E, Dicke M, Godfray HCJ, 2012. Insect symbionts as hidden players in insect-plant interactions. Trends in Ecology \& Evolution 27, 705-11. doi: 10.1016/j.tree.2012.08.013.

Franz A, Makkouk KM, Vetten HJ, 1998. Acquisition, retention and transmission of faba bean necrotic yellows virus by two of its aphid vectors, Aphis craccivora (Koch) and Acyrthosiphon pisum (Harris). Journal of Phytopathology 146, 347-55. doi: 10.1111/j.14390434.1998.tb04703.x.

Franz AWE, van der Wilk F, Verbeek M, 1999. Faba bean necrotic yellows virus (genus Nanovirus) requires a helper factor for its aphid transmission. Virology 262, 210-9. doi: 10.1006/viro.1999.9904.

Froussard $\mathrm{P}, 1992$. A random-PCR method ( $r P C R$ ) to construct whole cDNA library from low amounts of RNA. Nucleic Acids Research 20, 2900. doi: 10.1093/nar/20.11.2900.

Fu ZF, 2005. Genetic comparison of the rhabdoviruses from animals and plants. Current Topics in Microbiology and Immunology 292, 1-24.

Fuchs $M$, Schmitt-Keichinger C, Sanfaçon H, 2017. A renaissance in nepovirus research provides new insights into their molecular interface with hosts and vectors. In: Kielian $M$, Thomas $C M$, Marilyn JR, eds. Advances in Virus Research, Elsevier Science: Saint Louis. Vol 97, 61-105.

Fukatsu T, Tsuchida T, Nikoh N, 2001. Spiroplasma symbiont of the pea aphid, Acyrthosiphon pisum (Insecta: Homoptera). Applied and Environmental Microbiology 67, 1284-91. doi: 10.1128/AEM.67.3.1284-1291.2001.

Gaafar Y, Cordsen Nielsen G, Ziebell H, 2018a. Molecular characterisation of the first occurrence of Pea necrotic yellow dwarf virus in Denmark. New Disease Reports 37, 16. doi: 10.5197/j.2044-0588.2018.037.016.

Gaafar Y, Grausgruber-Gröger S, Ziebell H, 2016. Vicia faba V. sativa and Lens culinaris as new hosts for Pea necrotic yellow dwarf virus in Germany and Austria. New Disease Reports 34, 28. doi: 10.5197/j.2044-0588.2016.034.028.

Gaafar Y, Lüddecke P, Heidler C et al., 2019a. First report of Southern tomato virus in German tomatoes. New Disease Reports 40, 1. doi: 10.5197/j.2044-0588.2019.040.001.

Gaafar Y, Sieg-Müller A, Lüddecke P et al., 2019b. First report of natural infection of beetroot with Beet soil-borne virus. New Disease Reports 40, 5. doi: 10.5197/j.2044-0588.2019.040.005.

Gaafar Y, Sieg-Müller A, Lüddecke P et al., 2019c. First report of Turnip crinkle virus infecting garlic mustard (Alliaria petiolata) in Germany. New Disease Reports 39, 9. doi: 10.5197/j.20440588.2019.039.009.

Gaafar Y, Timchenko T, Ziebell H, 2017. First report of Pea necrotic yellow dwarf virus in The Netherlands. New Disease Reports 35, 23. doi: 10.5197/j.2044-0588.2017.035.023. 
Gaafar YZA, Abdelgalil MAM, Knierim D et al., 2018b. First report of physostegia chlorotic mottle virus on tomato (Solanum lycopersicum) in Germany. Plant Disease 102, 255. doi:

10.1094/PDIS-05-17-0737-PDN.

Gaafar YZA, Richert-Pöggeler KR, Maaß C, 2019d. Characterisation of a novel nucleorhabdovirus infecting alfalfa (Medicago sativa). Virology Journal 16, 113. doi: 10.1186/s12985-019-1147-3.

Gaafar YZA, Richert-Pöggeler KR, Sieg-Müller A et al., 2019e. A divergent strain of melon chlorotic spot virus isolated from black medic (Medicago lupulina) in Austria. Virology Journal 16, 297. doi: 10.1186/s12985-019-1195-8.

Gaafar YZA, Richert-Pöggeler KR, Sieg-Müller A et al., 2019f. Caraway yellows virus, a novel nepovirus from Carum carvi. Virology Journal 16, 529. doi: 10.1186/s12985-019-1181-1.

Gaafar YZA, Ziebell H, 2019a. Complete genome sequence of a highly divergent carrot torradovirus 1 strain from Apium graveolens. Archives of Virology. doi: 10.1007/s00705-01904272-3.

Gaafar YZA, Ziebell H, 2019b. Two divergent isolates of turnip yellows virus from pea and rapeseed and first report of turnip yellows virus-associated RNA in Germany. Microbiology Resource Announcements 8. doi: 10.1128/MRA.00214-19.

Gabrys B, Tjallingii WF, van Beek TA, 1997. Analysis of EPG recorded probing by cabbage aphid on host plant parts with different glucosinolate contents. Journal of Chemical Ecology 23, 1661-73. doi: 10.1023/B:JOEC.0000006442.56544.1a.

Galinier R, van Beurden S, Amilhat E et al., 2012. Complete genomic sequence and taxonomic position of eel virus European X (EVEX), a rhabdovirus of European eel. Virus Research 166, 112. doi: 10.1016/j.virusres.2012.02.020.

Gallet R, Kraberger S, Filloux D et al., 2018. Nanovirus-alphasatellite complex identified in Vicia cracca in the Rhône delta region of France. Archives of Virology 163, 695-700. doi: 10.1007/s00705-017-3634-4.

Garcla-Arenal F, Palukaitis P, 2009. Cucumber mosaic virus. In: van Regenmortel MHV, ed. Desk Encyclopedia of Plant and Fungal Virology, Elsevier professional: s.l., 171-6.

Garret A, Kerlan C, Thomas D, 1993. The intestine is a site of passage for potato leafroll virus from the gut lumen into the haemocoel in the aphid vector, Myzus persicae Sulz. Archives of Virology 131, 377-92. doi: 10.1007/bf01378639.

Garret A, Kerlan C, Thomas D, 1996. Ultrastructural study of acquisition and retention of potato leafroll luteovirus in the alimentary canal of its aphid vector, Myzus persicae Sulz. Archives of Virology 141, 1279-92. doi: 10.1007/BF01718830.

Garrett RG, 1991. Impact of viruses on pasture legume productivity. In: Proceedings of the Department of Agriculture, Victoria, white clover conference, 50-7.

Gentile M, Gelderblom HR, 2014. Electron microscopy in rapid viral diagnosis: an update. The New Microbiologica 37, 403-22.

Giakountis A, Skoufa A, Paplomatas El, 2015. Molecular characterization and phylogenetic analysis of a Greek lentil isolate of Pea seed-borne mosaic virus. Phytoparasitica 43, 615-28. doi: 10.1007/s12600-015-0495-9. 
Gildow FE, 1993. Evidence for receptor-mediated endocytosis regulating luteovirus acquisition by aphids. Phytopathology 83, 270. doi: 10.1094/Phyto-83-270.

Gildow FE, Reavy B, Mayo MA et al., 2000. Aphid acquisition and cellular transport of potato leafroll virus-like particles lacking P5 readthrough protein. Phytopathology 90, 1153-61. doi: 10.1094/PHYTO.2000.90.10.1153.

Ginestet C, 2011. ggplot2: elegant graphics for data analysis. Journal of the Royal Statistical Society: Series A (Statistics in Society) 174, 245-6. doi: 10.1111/j.1467-985X.2010.00676_9.x.

Goldsmith CS, Miller SE, 2009. Modern uses of electron microscopy for detection of viruses. Clinical Microbiology Reviews 22, 552-63. doi: 10.1128/CMR.00027-09.

Gómez-Gómez L, Boller T, 2000. FLS2: An LRR receptor-like kinase involved in the perception of the bacterial elicitor flagellin in Arabidopsis. Molecular Cell 5, 1003-11. doi: 10.1016/S10972765(00)80265-8.

Goodin MM, Jackson AO, 2002. Nucleorhabdovirus. In: Tidona CA, Darai G, Osmond CB, eds. The Springer index of viruses, Springer: Berlin, London, 1074-7.

Goodin MM, Min B-E, 2012. Virus-host protein interactions of plant-adapted rhabdoviruses. In: Dietzgen RG, Kuzmin IV, eds. Rhabdoviruses. Molecular taxonomy, evolution, genomics, ecology, host-vector interactions, cytopathology and control, Caister Academic Press: Norfolk, UK, 133-46.

Gorbalenya AE, Koonin EV, Donchenko AP, 1989. Two related superfamilies of putative helicases involved in replication, recombination, repair and expression of DNA and RNA genomes. Nucleic Acids Research 17, 4713-30.

Gorbalenya AE, Snijder EJ, 1996. Viral cysteine proteinases. Perspectives in Drug Discovery and Design 6, 64-86. doi: 10.1007/BF02174046.

Gottlieb Y, Zchori-Fein E, Mozes-Daube N et al., 2010. The transmission efficiency of tomato yellow leaf curl virus by the whitefly Bemisia tabaci is correlated with the presence of a specific symbiotic bacterium species. Journal of Virology 84, 9310-7. doi: 10.1128/JVI.0042310.

Graichen K, Rabenstein F, 1996. European isolates of beet western yellows virus (BWYV) from oilseed rape (Brassica napus L. ssp. napus) are non-pathogenic on sugar beet (Beta vulgaris $\mathrm{L}$ var. altissima) but represent isolates of turnip yellows virus (TuYV). Zeitschrift für Pflanzenkrankheiten und Pflanzenschutz / Journal of Plant Diseases and Protection 103, $233-$ 45.

Graichen K, Schliephake E, 1999. Infestation of winter oilseed rape by turnip yellows luteovirus and its effect on yield in Germany. In: 10 th International Rapeseed Congress - New Horizons from an Old Crop, 131-6.

Gray AM, Flatt PR, 1997. Pancreatic and extra-pancreatic effects of the traditional anti-diabetic plant, Medicago sativa (lucerne). British Journal of Nutrition 78, 325. doi: 10.1079/BJN19970150.

Gray S, Cilia M, Ghanim M, 2014. Circulative, "nonpropagative" virus transmission: an orchestra of virus-, insect-, and plant-derived instruments. Advances in Virus Research 89, 141-99. doi: 10.1016/B978-0-12-800172-1.00004-5. 
Gray S, Gildow FE, 2003. Luteovirus-aphid interactions. Annual Review of Phytopathology 41, 539-66. doi: 10.1146/annurev.phyto.41.012203.105815.

Grigoras I, Ginzo AldC, Martin DP et al., 2014. Genome diversity and evidence of recombination and reassortment in nanoviruses from Europe. The Journal of General Virology 95, 1178-91. doi: 10.1099/vir.0.063115-0.

Grigoras I, Gronenborn B, Vetten HJ, 2010a. First report of a nanovirus disease of pea in Germany. Plant Disease 94, 642. doi: 10.1094/PDIS-94-5-0642C.

Grigoras I, Timchenko T, Grande-Pérez A, 2010b. High variability and rapid evolution of a nanovirus. Journal of Virology 84, 9105-17. doi: 10.1128/JVI.00607-10.

Grigoras I, Timchenko T, Katul L, 2009. Reconstitution of authentic nanovirus from multiple cloned DNAs. Journal of Virology 83, 10778-87. doi: 10.1128/JVI.01212-09.

Grigoras I, Vetten H-J, Commandeur U, 2018. Nanovirus DNA-N encodes a protein mandatory for aphid transmission. Virology 522, 281-91. doi: 10.1016/j.virol.2018.07.001.

Grylls NE, Butler FC, 1956. An aphid transmitted virus affecting subterranean clover. Journal of the Australian Institute of Agricultural Science 22, 73-4.

Guo H, Sun Y, Li Y et al., 2014. Elevated CO2 alters the feeding behaviour of the pea aphid by modifying the physical and chemical resistance of Medicago truncatula. Plant, cell \& environment 37, 2158-68. doi: 10.1111/pce.12306.

Gutierrez AP, Morgan DJ, Havenstein DE, 1971. The ecology of Aphis craccivora Koch and subterranean clover stunt virus. I. The phenology of aphid populations and the epidemiology of virus in pastures in South-East Australia. The Journal of Applied Ecology 8, 699. doi: $10.2307 / 2402678$.

Guyatt KJ, Proll DF, Menssen A, 1996. The complete nucleotide sequence of bean yellow mosaic potyvirus RNA. Archives of Virology 141, 1231-46. doi: 10.1007/BF01718827.

Haan P de, Wagemakers L, Peters D, 1989. Molecular cloning and terminal sequence determination of the $S$ and M RNAs of tomato spotted wilt virus. The Journal of General Virology 70 (Pt 12), 3469-73. doi: 10.1099/0022-1317-70-12-3469.

Hagedorn DJ, Khan TN, 1984. Compendium of pea diseases. American Phytopathological Society St Paul, MN.

Hamilton RI, Edwardson JR, Francki RIB et al., 1981. Guidelines for the Identification and Characterization of Plant Viruses. The Journal of General Virology 54, 223-41. doi: 10.1099/0022-1317-54-2-223.

Hanley-Bowdoin L, Bejarano ER, Robertson D, 2013. Geminiviruses: masters at redirecting and reprogramming plant processes. Nature Reviews Microbiology 11, 777-88. doi: 10.1038/nrmicro3117.

Harris KF, 1977. An ingestion-egestion hypothesis of noncirculative virus transmission. In: Harris KF, Maramorosch K, eds. Aphids as virus vectors, Elsevier, 165-220.

Heydarnejad J, Kamali M, Massumi H et al., 2017. Identification of a nanovirus-alphasatellite complex in Sophora alopecuroides. Virus Research 235, 24-32. 
Hill JH, Whitham SA, 2014. Control of virus diseases in soybeans. Advances in Virus Research 90, 355-90. doi: 10.1016/B978-0-12-801246-8.00007-X.

Ho T, Tzanetakis IE, 2014. Development of a virus detection and discovery pipeline using next generation sequencing. Virology 471-473, 54-60. doi: 10.1016/j.virol.2014.09.019.

Hoang AT, Zhang H-M, Yang J et al., 2011. Identification, characterization, and distribution of Southern rice black-streaked dwarf virus in Vietnam. Plant Disease 95, 1063-9. doi: 10.1094/PDIS-07-10-0535.

Hogenhout SA, Ammar E-D, Whitfield AE, 2008. Insect vector interactions with persistently transmitted viruses. Annual Review of Phytopathology 46, 327-59. doi:

10.1146/annurev.phyto.022508.092135.

Houk EJ, Griffiths GW, 1980. Intracellular Symbiotes of the Homoptera. Annual Review of Entomology 25, 161-87. doi: 10.1146/annurev.en.25.010180.001113.

Hull R, 1969. Alfalfa mosaic virus. In: Smith KM, Lauffer MA, Bang FB, eds. Advances in Virus Research, Academic Press: New York. Vol 15, 365-433.

Hull R (ed), 2009. Comparative plant virology, 2nd edn. Amsterdam, Boston: Elsevier/Academic Press.

Hull R, 2014. Plant virology. Amsterdam: Academic Press.

Hulme PE, 2017. Climate change and biological invasions: evidence, expectations, and response options. Biological Reviews 92, 1297-313. doi: 10.1111/brv.12282.

lacobellis NS, Lo Cantore P, Capasso F, 2005. Antibacterial activity of Cuminum cyminum L. and Carum carvi L. essential oils. Journal of Agricultural and Food Chemistry 53, 57-61. doi: 10.1021/jf0487351.

Ido Y, Nakahara KS, Uyeda I, 2012. White clover mosaic virus-induced gene silencing in pea. Journal of General Plant Pathology 78, 127-32. doi: 10.1007/s10327-012-0360-3.

Idris A, Al-Saleh M, Piatek MJ, 2014. Viral metagenomics: analysis of begomoviruses by illumina high-throughput sequencing. Viruses 6, 1219-36. doi: 10.3390/v6031219.

Idris AM, Shahid MS, Briddon RW, 2011. An unusual alphasatellite associated with monopartite begomoviruses attenuates symptoms and reduces betasatellite accumulation. The Journal of General Virology 92, 706-17. doi: 10.1099/vir.0.025288-0.

Illumina. Sequencing Platforms | Compare NGS platform applications \& specifications. [https://www.illumina.com/systems/sequencing-platforms.html]. Accessed 10 August 2019.

Ip CLC, Loose M, Tyson JR et al., 2015. MinION Analysis and Reference Consortium: Phase 1 data release and analysis. F1000Research 4, 1075. doi: 10.12688/f1000research.7201.1.

Iseni F, Barge A, Baudin F, 1998. Characterization of rabies virus nucleocapsids and recombinant nucleocapsid-like structures. The Journal of General Virology 79 (Pt 12), 2909-19. doi: 10.1099/0022-1317-79-12-2909.

Ishikawa H, Yamaji M, 1985. Symbionin, an aphid endosymbiont-specific protein-I: Production of insects deficient in symbiont. Insect Biochemistry 15, 155-63. 
Isogai M, Tatuto N, Ujiie C, 2012. Identification and characterization of blueberry latent spherical virus, a new member of subgroup C in the genus Nepovirus. Archives of Virology 157, 297303. doi: 10.1007/s00705-011-1177-7.

Jackson AO, Dietzgen RG, Goodin MM, 2005. Biology of plant rhabdoviruses. Annual Review of Phytopathology 43, 623-60. doi: 10.1146/annurev.phyto.43.011205.141136.

Ji X-L, Yu N-T, Qu L, 2019. Banana bunchy top virus (BBTV) nuclear shuttle protein interacts and re-distributes BBTV coat protein in Nicotiana benthamiana. 3 Biotech 9, 121. doi: 10.1007/s13205-019-1656-1.

Jinn T-L, Stone JM, Walker JC, 2000. HAESA, an Arabidopsis leucine-rich repeat receptor kinase, controls floral organ abscission. Genes \& Development 14, 108-17.

Jo Y, Bae J-Y, Kim S-M, 2018a. Barley RNA viromes in six different geographical regions in Korea. Scientific Reports 8, 13237. doi: 10.1038/s41598-018-31671-4.

Jo Y, Lian S, Chu H et al., 2018b. Peach RNA viromes in six different peach cultivars. Scientific Reports 8, 1844. doi: 10.1038/s41598-018-20256-w.

Johne R, Müller H, Rector A, 2009. Rolling-circle amplification of viral DNA genomes using phi29 polymerase. Trends in Microbiology 17, 205-11. doi: 10.1016/j.tim.2009.02.004.

Johnstone GR, Mclean GD, 1987. Virus diseases of subterranean clover. Annals of Applied Biology 110, 421-40. doi: 10.1111/j.1744-7348.1987.tb03274.x.

Jones AT, Koenig R, Lesemann D-E, 1990. Serological comparison of isolates of cherry leaf roll virus from diseased beech and birch trees in a forest decline area in Germany with other isolates of the virus. Journal of Phytopathology 129, 339-44. doi: 10.1111/j.14390434.1990.tb04311.x.

Jones RAC, 2016. Future scenarios for plant virus pathogens as climate change progresses. Advances in Virus Research 95, 87-147. doi: 10.1016/bs.aivir.2016.02.004.

Kaiser WJ, 1982. Natural hosts and vectors of tobacco streak virus in Eastern Washington. Phytopathology 72, 1508. doi: 10.1094/Phyto-72-1508.

Kambara H, Takahashi S, 1993. Multiple-sheathflow capillary array DNA analyser. Nature 361, 565-6. doi: 10.1038/361565a0.

Kaplan IB, Lee L, Ripoll DR, 2007. Point mutations in the potato leafroll virus major capsid protein alter virion stability and aphid transmission. The Journal of General Virology 88, 1821-30. doi: 10.1099/vir.0.82837-0.

Katul L, 1992. Serological and molecular characterization of bean leaf roll virus (BLRV) and faba bean necrotic yellows virus (FBNYV). Ph. D. dissertation, University of Göttingen, Germany.

Kemper S, Schaack D, Schenck W von, 2019. AMI Markt Bilanz Getreide · Ölsaaten · Futtermittel 2019. [www.ami-informiert.de]. Accessed 8 July 2019.

Kesanakurti $P$, Belton $M$, Saeed $H, 2016$. Screening for plant viruses by next generation sequencing using a modified double strand RNA extraction protocol with an internal amplification control. Journal of Virological Methods 236, 35-40. doi:

10.1016/j.jviromet.2016.07.001. 
Khalifa ME, Pearson MN, 2013. Molecular characterization of three mitoviruses co-infecting a hypovirulent isolate of Sclerotinia sclerotiorum fungus. Virology 441, 22-30. doi:

10.1016/j.virol.2013.03.002.

Khetarpal RK, Maury Y, 1987. Pea seed-borne mosaic virus: a review. Agronomie 7, 215-24. doi: 10.1051/agro:19870401.

Kirby MJ, Guise CM, Adams AN, 2001. Comparison of bioassays and laboratory assays for apple stem grooving virus. Journal of Virological Methods 93, 167-73. doi: 10.1016/S01660934(01)00271-3.

Kircher M, Kelso J, 2010. High-throughput DNA sequencing--concepts and limitations. BioEssays 32, 524-36. doi: 10.1002/bies.200900181.

Kliot A, Ghanim M, 2013. The role of bacterial chaperones in the circulative transmission of plant viruses by insect vectors. Viruses 5, 1516-35. doi: 10.3390/v5061516.

Knierim D, Menzel W, Winter S, 2017. Analysis of the complete genome sequence of euphorbia ringspot virus, an atypical member of the genus Potyvirus. Archives of Virology 162, 291-3. doi: 10.1007/s00705-016-3087-1.

Knierim D, Menzel W, Winter S, 2019. Immunocapture of virions with virus-specific antibodies prior to high-throughput sequencing effectively enriches for virus-specific sequences. PLOS ONE 14, e0216713. doi: 10.1371/journal.pone.0216713.

Koenig R, Paul HL, 1982. Variants of ELISA in plant virus diagnosis. Journal of Virological Methods 5, 113-25.

Koloniuk I, Přibylová J, Fránová J, 2018. Molecular characterization and complete genome of a novel nepovirus from red clover. Archives of Virology 163, 1387-9. doi: 10.1007/s00705-0183742-9.

Kon T, Rojas MR, Abdourhamane IK, 2009. Roles and interactions of begomoviruses and satellite DNAs associated with okra leaf curl disease in Mali, West Africa. Journal of General Virology 90, 1001-13. doi: 10.1099/vir.0.008102-0.

Koren S, Walenz BP, Berlin K, 2017. Canu: scalable and accurate long-read assembly via adaptive k-mer weighting and repeat separation. Genome Research 27, 722-36. doi: 10.1101/gr.215087.116.

Kosugi S, Hasebe M, Tomita M, 2009. Systematic identification of cell cycle-dependent yeast nucleocytoplasmic shuttling proteins by prediction of composite motifs. Proceedings of the National academy of Sciences of the United States of America 106, 10171-6. doi: 10.1073/pnas.0900604106.

Kraft JM (ed), 2008. Compendium of pea diseases and pests, 2nd edn. St. Paul, Minn.: APS Press.

Krapp S, Greiner E, Amin B, 2017. The stress granule component G3BP is a novel interaction partner for the nuclear shuttle proteins of the nanovirus pea necrotic yellow dwarf virus and geminivirus abutilon mosaic virus. Virus Research 227, 6-14. doi:

10.1016/j.virusres.2016.09.021.

Krenz B, SchießI I, Greiner E, 2017. Analyses of pea necrotic yellow dwarf virus-encoded proteins. Virus Genes 53, 454-63. doi: 10.1007/s11262-017-1439-x. 
Kreuze JF, Perez A, Untiveros M et al., 2009. Complete viral genome sequence and discovery of novel viruses by deep sequencing of small RNAs: a generic method for diagnosis, discovery and sequencing of viruses. Virology 388, 1-7. doi: 10.1016/j.virol.2009.03.024.

Kumar LM, Foster JA, McFarland C, 2018a. First report of Barley virus $G$ in switchgrass (Panicum virgatum). Plant Disease 102, 466. doi: 10.1094/PDIS-09-17-1390-PDN.

Kumar S, Stecher G, Li M, 2018b. MEGA X: molecular evolutionary genetics analysis across computing platforms. Molecular Biology and Evolution 35, 1547-9. doi: 10.1093/molbev/msy096.

Kumar S, Stecher G, Tamura K, 2016. MEGA7: Molecular evolutionary genetics analysis version 7.0 for bigger datasets. Molecular Biology and Evolution 33, 1870-4. doi: $10.1093 / \mathrm{molbev} / \mathrm{msw} 054$.

Kumari SG, Attar N, Mustafayev E, 2009. First report of Faba bean necrotic yellows virus affecting legume crops in Azerbaijan. Plant Disease 93, 1220. doi: 10.1094/PDIS-93-11-1220C.

Kumari SG, Makkouk KM, 2007. Virus diseases of faba bean (Vicia faba L.) in Asia and Africa. Plant Viruses 1, 93-105.

Kumari SG, Rodoni B, Vetten H-J et al., 2010. Detection and partial characterization of Milk vetch dwarf virus isolates from faba bean (Vicia faba L.) in Yunnan Province, China. Journal of Phytopathology 158, 35-9. doi: 10.1111/j.1439-0434.2009.01572.x.

La Cour T, Kiemer L, Mølgaard A, 2004. Analysis and prediction of leucine-rich nuclear export signals. Protein Engineering, Design \& Selection 17, 527-36. doi: 10.1093/protein/gzh062.

Laporte C, Vetter G, Loudes A-M et al., 2003. Involvement of the secretory pathway and the cytoskeleton in intracellular targeting and tubule assembly of Grapevine fanleaf virus movement protein in tobacco BY-2 cells. The Plant Cell Online 15, 2058-75. doi: 10.1105/tpc.013896.

Larkin MA, Blackshields G, Brown NP et al., 2007. Clustal W and Clustal X version 2.0. Bioinformatics (Oxford, England) 23, 2947-8. doi: 10.1093/bioinformatics/btm404.

Lartey RT, Lane LC, Melcher U, 1994. Electron microscopic and molecular characterization of turnip vein-clearing virus. Archives of Virology 138, 287-98. doi: 10.1007/BF01379132.

Latham LJ, Jones RAC, 2001. Alfalfa mosaic and pea seed-borne mosaic viruses in cool season crop, annual pasture, and forage legumes: susceptibility, sensitivity, and seed transmission. Australian Journal of Agricultural Research 52, 771. doi: 10.1071/AR00165.

Le Gall O, Candresse T, Dunez J, 1995. A multiple alignment of the capsid protein sequences of nepoviruses and comoviruses suggests a common structure. Archives of Virology 140, 204153. doi: 10.1007/BF01322691.

Lecoq H, Wipf-Scheibel C, Verdin E, 2019. Characterization of the first tenuivirus naturally infecting dicotyledonous plants. Archives of Virology 164, 297-301. doi: 10.1007/s00705-0184057-6.

Lee L, Kaplan IB, Ripoll DR, 2005. A surface loop of the potato leafroll virus coat protein is involved in virion assembly, systemic movement, and aphid transmission. Journal of Virology 79, 1207-14. doi: 10.1128/JVI.79.2.1207-1214.2005. 
Leff JW, Lynch RC, Kane NC, 2017. Plant domestication and the assembly of bacterial and fungal communities associated with strains of the common sunflower, Helianthus annuus. The New Phytologist 214, 412-23. doi: 10.1111/nph.14323.

Lesker T, Rabenstein F, Maiss E, 2013. Molecular characterization of five betacryptoviruses infecting four clover species and dill. Archives of Virology 158, 1943-52. doi: 10.1007/s00705013-1691-x.

Levene MJ, Korlach J, Turner SW, 2003. Zero-mode waveguides for single-molecule analysis at high concentrations. Science (New York, N.Y.) 299, 682-6. doi: 10.1126/science.1079700.

Li C-X, Shi M, Tian J-H et al., 2015. Unprecedented genomic diversity of RNA viruses in arthropods reveals the ancestry of negative-sense RNA viruses. eLife 4. doi: 10.7554/eLife.05378.

Li H, Homer N, 2010. A survey of sequence alignment algorithms for next-generation sequencing. Briefings in Bioinformatics 11, 473-83. doi: 10.1093/bib/bbq015.

Li H, Liu X, Liu X et al., 2018. Host plant Infection by soybean mosaic virus reduces the fitness of its vector, Aphis glycines (Hemiptera: Aphididae). Journal of Economic Entomology 111, 201723. doi: 10.1093/jee/toy165.

Li R, Gao S, Hernandez AG, 2012. Deep sequencing of small RNAs in tomato for virus and viroid identification and strain differentiation. PLOS ONE 7, e37127. doi: 10.1371/journal.pone.0037127.

Li Y, Lu J, Han Y, 2013. RNA interference functions as an antiviral immunity mechanism in mammals. Science (New York, N.Y.) 342, 231-4. doi: 10.1126/science.1241911.

Li Z, Yu M, Zhang H, 2005. Improved rapid amplification of cDNA ends (RACE) for mapping both the 5 ' and 3 ' terminal sequences of paramyxovirus genomes. Journal of Virological Methods 130, 154-6. doi: 10.1016/j.jviromet.2005.06.022.

Lister RM, 1958. Some turnip viruses in Scotland and their effect on yield. Plant Pathology 7 , 144-6. doi: 10.1111/j.1365-3059.1958.tb00854.x.

Liu B, Preisser EL, Chu D et al., 2013. Multiple forms of vector manipulation by a plant-infecting virus: Bemisia tabaci and tomato yellow leaf curl virus. Journal of Virology 87, 4929-37. doi: 10.1128/JVI.03571-12.

Liu L, Li Y, Li S et al., 2012. Comparison of next-generation sequencing systems. Journal of Biomedicine \& Biotechnology 2012, 251364. doi: 10.1155/2012/251364.

Liu X, Gorovsky MA, 1993. Mapping the $5^{\prime}$ and $3^{\prime}$ ends of Tetrahymena thermophila mRNAs using RNA ligase mediated amplification of CDNA ends (RLM-RACE). Nucleic Acids Research 21, 4954-60. doi: 10.1093/nar/21.21.4954.

Liu Y, Du Z, Wang H, 2018. Identification and characterization of wheat yellow striate virus, a novel leafhopper-transmitted nucleorhabdovirus infecting wheat. Frontiers in Microbiology 9 , 468. doi: 10.3389/fmicb.2018.00468.

Lockhart BE, Swenson AS, Olszewski NE, 2008. Characterization of a strain of Turnip vein-clearing virus causing red ringspot of penstemon. Plant Disease 92, 725-9. doi: 10.1094/PDIS-92-50725. 
Longdon B, Obbard DJ, Jiggins FM, 2010. Sigma viruses from three species of Drosophila form a major new clade in the rhabdovirus phylogeny. Proceedings of the Royal Society of London B: Biological Sciences 277, 35-44. doi: 10.1098/rspb.2009.1472.

Lu H, Giordano F, Ning Z, 2016. Oxford Nanopore MinION sequencing and genome assembly. Genomics, Proteomics \& Bioinformatics 14, 265-79. doi: 10.1016/j.gpb.2016.05.004.

Lu L, Wu S, Jiang J, 2018. Whole genome deep sequencing revealed host impact on population structure, variation and evolution of Rice stripe virus. Virology 524, 32-44. doi: 10.1016/j.virol.2018.08.005.

MacDiarmid R, Rodoni B, Melcher U, 2013. Biosecurity implications of new technology and discovery in plant virus research. PLoS pathogens 9, e1003337. doi: 10.1371/journal.ppat.1003337.

Makkouk KM, Kumari SG, van Leur JAG, 2014. Control of plant virus diseases in cool-season grain legume crops. Advances in Virus Research 90, 207-53. doi: 10.1016/B978-0-12-8012468.00004-4.

Makkouk KM, Rizkallah L, Madkour M et al., 1994. Survey of faba bean (Vicia faba L.) for viruses in Egypt. Phytopathologia Mediterranea 33, 207-11.

Makkouk KM, Vetten HJ, Katul L, 1998. Epidemiology and control of faba bean necrotic yellows virus. In: Hadidi A, Khetarpal RK, Koganezawa H, eds. Plant virus disease control, APS Press: St. Paul, Minn., 534-40.

Mar TB, Mendes IR, Lau D et al., 2017. Interaction between the new world begomovirus Euphorbia yellow mosaic virus and its associated alphasatellite: effects on infection and transmission by the whitefly Bemisia tabaci. The Journal of General Virology 98, 1552-62. doi: 10.1099/jgv.0.000814.

Marchler-Bauer A, Bo Y, Han L et al., 2017. CDD/SPARCLE: functional classification of proteins via subfamily domain architectures. Nucleic Acids Research 45, D200-D203. doi: 10.1093/nar/gkw1129.

Mardis ER, 2008. The impact of next-generation sequencing technology on genetics. Trends in Genetics 24, 133-41. doi: 10.1016/j.tig.2007.12.007.

Maree HJ, Fox A, Al Rwahnih M, 2018. Application of HTS for routine plant virus diagnostics: state of the art and challenges. Frontiers in Plant Science 9, 1082. doi: 10.3389/fpls.2018.01082.

Margulies M, Egholm M, Altman WE et al., 2005. Genome sequencing in microfabricated highdensity picolitre reactors. Nature 437, 376-80. doi: 10.1038/nature03959.

Mariano AC, Andrade MO, Santos AA et al., 2004. Identification of a novel receptor-like protein

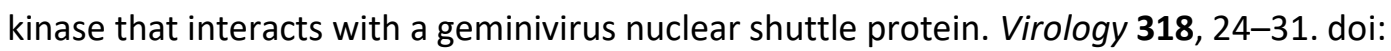
10.1016/j.virol.2003.09.038.

Markarian N, Li HW, Ding SW, 2004. RNA silencing as related to viroid induced symptom expression. Archives of Virology 149, 397-406. doi: 10.1007/s00705-003-0215-5.

Marston HR, Quinlan-Watson F, Dewey DW, 1943. The dehydration of lucerne (Medicago sativa) and its potentialities as a concentrated source of ascorbic acid and of carotene for human 
consumption. Journal of the Council for Scientific and Industrial Research, Australia 16, 11324.

Massart S, Candresse T, Gil J et al., 2017. A framework for the evaluation of biosecurity, commercial, regulatory, and scientific impacts of plant viruses and viroids identified by NGS technologies. Frontiers in Microbiology 8. doi: 10.3389/fmicb.2017.00045.

Massart S, Chiumenti M, Jonghe K de et al., 2019. Virus detection by high-throughput sequencing of small RNAs: large-scale performance testing of sequence analysis strategies. Phytopathology 109, 488-97. doi: 10.1094/PHYTO-02-18-0067-R.

Massart S, Olmos A, Jijakli H, 2014. Current impact and future directions of high throughput sequencing in plant virus diagnostics. Virus Research 188, 90-6. doi: 10.1016/j.virusres.2014.03.029.

Mayo MA, 2002. Virology division news: ICTV at the Paris ICV: Results of the plenary session and the binomial ballot. Archives of Virology 147, 2254-60. doi: 10.1007/s007050200052.

McKenzie DL, Morrall RAA, 1975. Diseases of specialty crops in Saskatchewan: II. Notes on field pea in 1973-74 and on lentil in 1973. Canadian Plant Disease Survey 55, 97-100.

Mclean DL, Kinsey MG, 1964. A technique for electronically recording aphid feeding and salivation. Nature 202, 1358-9. doi: 10.1038/2021358a0.

Mclean DL, Kinsey MG, 1965. Identification of electrically recorded curve patterns associated with aphid salivation and ingestion. Nature 205, 1130-1. doi: 10.1038/2051130a0.

McLean AHC, van Asch M, Ferrari J, 2011. Effects of bacterial secondary symbionts on host plant use in pea aphids. Proceedings: Biological Sciences 278, 760-6. doi: 10.1098/rspb.2010.1654.

Menzel W, Knierim D, Richert-Pöggeler KR, 2016. Characterization of a nucleorhabdovirus from Physostegia. Julius-Kühn-Archiv 454, 283-4. doi: 10.5073/JKA.2016.454.000.

Metzker ML, 2010. Sequencing technologies - the next generation. Nature reviews. Genetics 11, 31-46. doi: 10.1038/nrg2626.

Miller JR, Koren S, Sutton G, 2010. Assembly algorithms for next-generation sequencing data. Genomics 95, 315-27. doi: 10.1016/j.ygeno.2010.03.001.

Milne RG, 1984. Electron microscopy for the identification of plant viruses in in vitro preparations. In: Maramorosch K, Koprowski H, eds. Methods in Virology, Academic Press: New York, London. Vol 7, 87-120.

Milne RG, Lesemann D-E, 1984. Immunosorbent electron microscopy in plant virus studies. In: Maramorosch K, Koprowski H, eds. Methods in Virology, Academic Press: New York, London. Vol 8, 85-101.

Mollov D, Maroon-Lango C, Kuniata L, 2016. Detection by next generation sequencing of a multisegmented viral genome from sugarcane associated with Ramu stunt disease. Virus Genes 52, 152-5. doi: 10.1007/s11262-015-1279-5.

Moran NA, Russell JA, Koga R, 2005. Evolutionary relationships of three new species of Enterobacteriaceae living as symbionts of aphids and other insects. Applied and Environmental Microbiology 71, 3302-10. doi: 10.1128/AEM.71.6.3302-3310.2005. 
Moran PJ, Thompson GA, 2001. Molecular responses to aphid feeding in Arabidopsis in relation to plant defense pathways. Plant Physiology 125, 1074-85. doi: 10.1104/pp.125.2.1074.

Morris TJ, 1979. Isolation and analysis of double-stranded RNA from virus-infected plant and fungal tissue. Phytopathology 69, 854. doi: 10.1094/Phyto-69-854.

Munson MA, Baumann P, Kinsey MG, 1991. Buchnera gen. nov. and Buchnera aphidicola sp. nov., a taxon consisting of the mycetocyte-associated, primary endosymbionts of aphids. International Journal of Systematic Bacteriology 41, 566-8. doi: 10.1099/00207713-41-4-566.

Mushegian AR, 1994. The putative movement domain encoded by nepovirus RNA-2 is conserved in all sequenced nepoviruses. Archives of Virology 135, 437-41. doi: 10.1007/BF01310028.

Musil M, 1966. Uber das Vorkommen des Virus des Blattrollens der Erbse in der Siowakei. (On the occurrence of a leaf roll virus in pea in Slovakia.). Biologia, Bratislava 21, 133-8.

Myers SS, Smith MR, Guth S et al., 2017. Climate change and global food systems: potential impacts on food security and undernutrition. Annual Review of Public Health 38, 259-77.

Naidu RA, Hughes JDA, 2003. Methods for the detection of plant virus diseases. Plant Virology in Sub Saharan Africa, 233-53.

Nakabayashi H, Yamaji Y, Kagiwada S, 2002. The complete nucleotide sequence of a Japanese isolate of white clover mosaic virus strain RC. Journal of General Plant Pathology 68, 173-6. doi: 10.1007/PL00013072.

Nakazono-Nagaoka E, Takahashi T, Shimizu T et al., 2009. Cross-protection against bean yellow mosaic virus (BYMV) and clover yellow vein virus by Attenuated BYMV isolate M11. Phytopathology 99, 251-7. doi: 10.1094/PHYTO-99-3-0251.

Nandakishor HV, Kumaraswamy B, Mane SS, 2017. Host range studies of soybean mosaic virus. International Journal of Current Microbiology and Applied Sciences 6, 304-8. doi: 10.20546/ijcmas.2017.607.035.

Narzisi G, Mishra B, 2011. Comparing de novo genome assembly: the long and short of it. PLOS ONE 6, e19175. doi: 10.1371/journal.pone.0019175.

Nault LR, 1997. Arthropod transmission of plant viruses. A new synthesis. Annals of the Entomological Society of America 90, 521-41. doi: 10.1093/aesa/90.5.521.

Nawaz-UI-Rehman MS, Nahid N, Mansoor S, 2010. Post-transcriptional gene silencing suppressor activity of two non-pathogenic alphasatellites associated with a begomovirus. Virology 405, 300-8. doi: 10.1016/j.virol.2010.06.024.

Ng JCK, Falk BW, 2006. Virus-vector interactions mediating nonpersistent and semipersistent transmission of plant viruses. Annual Review of Phytopathology 44, 183-212. doi: 10.1146/annurev.phyto.44.070505.143325.

Ng JCK, Perry KL, 2004. Transmission of plant viruses by aphid vectors. Molecular Plant Pathology 5, 505-11. doi: 10.1111/j.1364-3703.2004.00240.x.

Nibert ML, Vong M, Fugate KK, 2018. Evidence for contemporary plant mitoviruses. Virology 518, 14-24. doi: 10.1016/j.virol.2018.02.005. 
Ohtaka C, 1991. Effects of heat treatment on the symbiotic system of an aphid mycetocyte. Symbiosis 11, 19-30.

Okada R, Kiyota E, Moriyama H, 2015. A simple and rapid method to purify viral dsRNA from plant and fungal tissue. Journal of General Plant Pathology 81, 103-7. doi: 10.1007/s10327014-0575-6.

$\varnothing$ kland AL, Skoge RH, Nylund A, 2018. The complete genome sequence of CrRV-Ch01, a new member of the family Rhabdoviridae in the parasitic copepod Caligus rogercresseyi present on farmed Atlantic salmon (Salmo salar) in Chile. Archives of Virology, 1657-61. doi: 10.1007/s00705-018-3768-z.

Oliveros JC, 2015. Venny. An interactive tool for comparing lists with Venn's diagrams. [https://bioinfogp.cnb.csic.es/tools/venny/index.html].

Olmos A, Boonham N, Candresse T et al., 2018. High-throughput sequencing technologies for plant pest diagnosis: challenges and opportunities. EPPO Bulletin 48, 219-24. doi: 10.1111/epp.12472.

Olson J, Rebek E, Schnelle M, 2017. Rose rosette disease. [http://factsheets.okstate.edu/documents/epp-7329-rose-rosette-disease/]. Accessed 18 October 2019.

Ortiz V, Navarro E, Castro S, 2006. Incidence and transmission of faba bean necrotic yellows virus (FBNYV) in Spain. Spanish Journal of Agricultural Research 4, 255. doi: 10.5424/sjar/2006043200.

Padmanabhan C, Zheng Y, Li R, 2015. Complete Genome Sequence of Southern tomato virus Naturally Infecting Tomatoes in Bangladesh. Genome Announcements 3. doi: 10.1128/genomeA.01522-15.

Palukaitis P, Roossinck MJ, Dietzgen RG, 1992. Cucumber mosaic virus. In: Maramorosch K, Murphy FA, Shatkin Aaron J., eds. Advances in Virus Research, Elsevier. Vol 41, 281-348.

Pandey B, Naidu RA, Grove GG, 2018. Detection and analysis of mycovirus-related RNA viruses from grape powdery mildew fungus Erysiphe necator. Archives of Virology 163, 1019-30. doi: 10.1007/s00705-018-3714-0.

Paprotka T, Metzler V, Jeske H, 2010. The first DNA 1-like alpha satellites in association with New World begomoviruses in natural infections. Virology 404, 148-57. doi: 10.1016/j.virol.2010.05.003.

Park CY, Lee S-H, Lim S, 2017. First report of white clover mosaic virus on white clover (Trifolium repens) in Korea. Plant Disease 101, 1559. doi: 10.1094/PDIS-02-17-0256-PDN.

Patil BL, Kumar PL, 2015. Pigeonpea sterility mosaic virus: a legume-infecting Emaravirus from South Asia. Molecular Plant Pathology 16, 775-86. doi: 10.1111/mpp.12238.

Patil BL, Legg JP, Kanju E, 2015. Cassava brown streak disease: a threat to food security in Africa. The Journal of General Virology 96, 956-68. doi: 10.1099/vir.0.000014.

Pechinger K, Chooi KM, MacDiarmid RM, 2019. A new era for mild strain cross-protection. Viruses 11. doi: 10.3390/v11070670. 
Pecman A, Kutnjak D, Gutiérrez-Aguirre l et al., 2017. Next generation sequencing for detection and discovery of plant viruses and viroids: comparison of two approaches. Frontiers in Microbiology 8, 1998. doi: 10.3389/fmicb.2017.01998.

Peoples MB, Bowman AM, Gault RR et al., 2001. Factors regulating the contributions of fixed nitrogen by pasture and crop legumes to different farming systems of eastern Australia. Plant and Soil 228, 29-41. doi: 10.1023/A:1004799703040.

Pescod KV, Quick WP, Douglas AE, 2007. Aphid responses to plants with genetically manipulated phloem nutrient levels. Physiological Entomology 32, 253-8. doi: 10.1111/j.13653032.2007.00577.x.

Peter KA, Liang D, Palukaitis $P, 2008$. Small deletions in the potato leafroll virus readthrough protein affect particle morphology, aphid transmission, virus movement and accumulation. The Journal of General Virology 89, 2037-45. doi: 10.1099/vir.0.83625-0.

Phibbs A, Barta A, Domier LL, 2004. First report of Soybean dwarf virus on soybean in Wisconsin. Plant Disease 88, 1285. doi: 10.1094/PDIS.2004.88.11.1285A.

Prado E, Tjallingii WF, 1994. Aphid activities during sieve element punctures. Entomologia Experimentalis et Applicata 72, 157-65. doi: 10.1111/j.1570-7458.1994.tb01813.x.

Purdy LH, 1979. Sclerotinia sclerotiorum: History, diseases and symptomatology, host range, geographic distribution, and impact. Phytopathology 69, 875. doi: 10.1094/Phyto-69-875.

Quantz L, Volk J, 1954. Die blattrollkrankheit der ackerbohne und erbse, eine neue viruskrankheit bei leguminosen. Nachrichtenblatt des Deutschen Pflanzenschutzdienstes $\mathbf{6}$, 177-82.

R Core Team, 2014. $R$ : a language and environment for statistical computing. Vienna, Austria: R Foundation for Statistical Computing, Vienna, Austria.

R Core Team, 2019. R: a language and environment for statistical computing. Vienna, Austria: $R$ Foundation for Statistical Computing, Vienna, Austria.

Rebenstorf K, 2005. Untersuchungen zur Epidemiologie des Cherry leaf roll virus (CLRV) genetische und serologische Diversität in Abhängigkeit von der Wirtspflanzenart und der geographischen Herkunft. Humboldt-Universität zu Berlin. PhD thesis.

Reddy MV, Nene YL, Verma JP, 1979. Pea leaf roll virus causes chickpea stunt. International Chickpea Newsletter 1, 8.

Reuter JA, Spacek DV, Snyder MP, 2015. High-throughput sequencing technologies. Molecular Cell 58, 586-97. doi: 10.1016/j.molcel.2015.05.004.

Richert-Pöggeler KR, Franzke K, Hipp K, 2018. Electron microscopy methods for virus diagnosis and high resolution analysis of viruses. Frontiers in Microbiology 9, 3255. doi: 10.3389/fmicb.2018.03255.

Rizos H, Gunn LV, Pares RD, 1992. Differentiation of cucumber mosaic virus isolates using the polymerase chain reaction. Journal of General Virology 73 (Pt 8), 2099-103. doi:

10.1099/0022-1317-73-8-2099. 
Roberts IM, Harrison BD, 1979. Detection of potato leafroll and potato mop-top viruses by immunosorbent electron microscopy. Annals of Applied Biology 93, 289-97. doi: 10.1111/j.1744-7348.1979.tb06544.x.

Roberts JMK, Ireland KB, Tay WT, 2018. Honey bee-assisted surveillance for early plant virus detection. Annals of Applied Biology 173, 285-93. doi: 10.1111/aab.12461.

Rochon D, Lommel S, Martelli GP, 2012. Family Tombusviridae. In: King AMQ, Adams MJ, Carstens EB, Lefkowitz EJ, eds. Virus taxonomy. Classification and nomenclature of viruses. Ninth report of the International Committee on Taxonomy of Viruses, Elsevier academic press: London, Waltham MA, 1111-38.

Roggero P, Ciuffo M, Vaira AM, 2000. An Ophiovirus isolated from lettuce with big-vein symptoms. Archives of Virology 145, 2629-42. doi: 10.1007/s007050070012.

Romanovskaya A, Sarin LP, Bamford DH, 2013. High-throughput purification of double-stranded RNA molecules using convective interaction media monolithic anion exchange columns. Journal of Chromatography. A 1278, 54-60. doi: 10.1016/j.chroma.2012.12.050.

Roossinck MJ, 2011. The good viruses: viral mutualistic symbioses. Nature reviews. Microbiology 9, 99-108. doi: 10.1038/nrmicro2491.

Roossinck MJ, 2017. Deep sequencing for discovery and evolutionary analysis of plant viruses. Virus Research 239, 82-6. doi: 10.1016/j.virusres.2016.11.019.

Roossinck MJ, Martin DP, Roumagnac $P, 2015$. Plant virus metagenomics: advances in virus discovery. Phytopathology 105, 716-27. doi: 10.1094/PHYTO-12-14-0356-RVW.

Roossinck MJ, Saha P, Wiley GB et al., 2010. Ecogenomics. Using massively parallel pyrosequencing to understand virus ecology. Molecular Ecology 19 Suppl 1, 81-8. doi: 10.1111/j.1365-294X.2009.04470.x.

Roossinck MJ, Sleat D, Palukaitis P, 1992. Satellite RNAs of plant viruses: structures and biological effects. Microbiology and Molecular Biology Reviews 56, 265-79.

Ross JP, 1977. Effect of aphid-transmitted soybean mosaic virus on yields of closely related resistant and susceptible soybean lines. Crop Science 17, 869. doi: 10.2135/cropsci1977.0011183X001700060014x.

Rothberg JM, Hinz W, Rearick TM et al., 2011. An integrated semiconductor device enabling nonoptical genome sequencing. Nature 475, 348-52. doi: 10.1038/nature10242.

Rott $M$, Xiang $Y$, Boyes I et al., 2017. Application of next generation sequencing for diagnostic testing of tree fruit viruses and viroids. Plant Disease 101, 1489-99. doi: 10.1094/PDIS-03-170306-RE.

Rott ME, Kesanakurti P, Berwarth C et al., 2018. Discovery of negative-sense RNA viruses in trees infected with apple rubbery wood disease by next-generation sequencing. Plant Disease 102, 1254-63. doi: 10.1094/PDIS-06-17-0851-RE.

Rozado-Aguirre Z, Adams I, Collins L, 2016. Detection and transmission of carrot torrado virus, a novel putative member of the Torradovirus genus. Journal of Virological Methods 235, 11924. doi: 10.1016/j.jviromet.2016.05.018. 
Russell AC, 1994. Three new pulse cultivars for New Zealand's arable industry. In: Proceedings Agronomy Society of N.Z., 125-8.

Sabanadzovic S, Valverde RA, Brown JK, 2009. Southern tomato virus: The link between the families Totiviridae and Partitiviridae. Virus Research 140, 130-7. doi: 10.1016/j.virusres.2008.11.018.

Samac DA, Rhodes LH, Lamp WO (eds), 2016. Compendium of alfalfa diseases and pests. St. Paul, Minnesota, USA: The American Phytopathological Society.

Sanfaçon H, Wellink J, Le Gall O, 2009. Secoviridae: a proposed family of plant viruses within the order Picornavirales that combines the families Sequiviridae and Comoviridae, the unassigned genera Cheravirus and Sadwavirus, and the proposed genus Torradovirus. Archives of Virology 154, 899-907. doi: 10.1007/s00705-009-0367-z.

Sanger F, Nicklen S, Coulson AR, 1977. DNA sequencing with chain-terminating inhibitors. Proceedings of the National Academy of Sciences of the United States of America 74, 5463-7. doi: 10.1073/pnas.74.12.5463.

Sanger M, Passmore B, Falk BW, 1994. Symptom severity of beet western yellows virus strain ST9 is conferred by the ST9-associated RNA and is not associated with virus release from the phloem. Virology 200, 48-55. doi: 10.1006/viro.1994.1161.

Sanjuán R, 2010. Mutational fitness effects in RNA and single-stranded DNA viruses: common patterns revealed by site-directed mutagenesis studies. Philosophical Transactions of the Royal Society of London. Series B, Biological Sciences 365, 1975-82. doi: 10.1098/rstb.2010.0063.

Sano Y, Wada M, Hashimoto Y, 1998. Sequences of ten circular ssDNA components associated with the milk vetch dwarf virus genome. The Journal of General Virology 79 (Pt 12), 3111-8. doi: 10.1099/0022-1317-79-12-3111.

Santos AA, Carvalho CM, Florentino LH, 2009. Conserved threonine residues within the A-loop of the receptor NIK differentially regulate the kinase function required for antiviral signaling. PLOS ONE 4, e5781. doi: 10.1371/journal.pone.0005781.

Santos AA, Lopes KVG, Apfata JAC, 2010. NSP-interacting kinase, NIK: a transducer of plant defence signalling. Journal of experimental botany 61, 3839-45. doi: 10.1093/jxb/erq219.

Saucke $H$, Uteau D, Brinkmann K, 2019. Symptomology and yield impact of pea necrotic yellow dwarf virus (PNYDV) in faba bean (Vicia faba L. minor). European Journal of Plant Pathology 153, 1299-315. doi: 10.1007/s10658-018-01643-5.

Sauge M-H, Lacroze J-P, Poessel J-L, 2002. Induced resistance by Myzus persicae in the peach cultivar 'Rubira'. Entomologia Experimentalis et Applicata 102, 29-37. doi: 10.1046/j.15707458.2002.00922.x.

Schliephake E, Habekuss A, Scholz M, 2013. Barley yellow dwarf virus transmission and feeding behaviour of Rhopalosiphum padi on Hordeum bulbosum clones. Entomologia Experimentalis et Applicata 146, 347-56. doi: 10.1111/eea.12033.

Schmidt HE, 1981. The diagnosis of viruses of legume crops in German Democratic Republic as a prerequisite for breeding broad bean (Vicia faba L.), bean (Phaseolus vulgaris L.), pea (Pisum 
$s p$. ) and lupin (Lupinus sp.) for resistance to virus diseases. In: Principles of plant resistance to diseases and pests. Research Institute Plant Protection, 25-33.

Schwarzkopf A, Rosenberger D, Niebergall M, 2013. To feed or not to feed: plant factors located in the epidermis, mesophyll, and sieve elements influence pea aphid's ability to feed on legume species. PLOS ONE 8, e75298. doi: 10.1371/journal.pone.0075298.

Seifers DL, Harvey TL, Martin TJ et al., 2005. Association of a virus with wheat displaying yellow head disease symptoms in the great plains. Plant Disease 89, 888-95. doi: 10.1094/PD-890888.

Shahjahan RM, Hughes KJ, Leopold RA, 1995. Lower incubation temperature increases yield of insect genomic DNA isolated by the CTAB method. BioTechniques 19, 332-4.

Shi M, Lin X-D, Tian J-H et al., 2016a. Redefining the invertebrate RNA virosphere. Nature 540, 539-43. doi: 10.1038/nature20167.

Shi M, Lin X-D, Vasilakis $\mathrm{N}$ et al., 2016b. Divergent viruses discovered in arthropods and vertebrates revise the evolutionary history of the Flaviviridae and related viruses. Journal of Virology 90, 659-69. doi: 10.1128/JVI.02036-15.

Shirako Y, Falk BW, Haenni AL, 2012. Genus Tenuivirus. In: King AMQ, Adams MJ, Carstens EB, Lefkowitz EJ, eds. Virus taxonomy: ninth report of the international committee on taxonomy of viruses, Elsevier Academic Press San Diego, 771-6.

Sicard A, Yvon M, Timchenko T et al., 2013. Gene copy number is differentially regulated in a multipartite virus. Nature Communications 4, 2248. doi: 10.1038/ncomms3248.

Sicard A, Zeddam J-L, Yvon M, 2015. Circulative nonpropagative aphid transmission of nanoviruses: an oversimplified view. Journal of Virology 89, 9719-26. doi: 10.1128/JVI.0078015.

Simons JN, 1953. Vector-virus relationships of pea-enation mosaic and the pea aphid Macrosiphum pisi (Kalt.). University of California, Berkeley.

Srinivasan R, Alvarez JM, 2007. Effect of Mixed Viral Infections (Potato Virus Y-Potato Leafroll Virus) on Biology and Preference of Vectors Myzus persicae and Macrosiphum euphorbiae (Hemiptera: Aphididae). Journal of Economic Entomology 100, 646-55. doi: 10.1093/jee/100.3.646.

Stevens M, Lacomme C, 2017. Transmission of plant viruses. In: van Emden HF, Harrington R, eds. Aphids as crop pests, CABI: Wallingford, 323-61.

Stevens M, Smith HG, Hallsworth PB, 1994. The host range of beet yellowing viruses among common arable weed species. Plant Pathology 43, 579-88. doi: 10.1111/j.13653059.1994.tb01593.x.

Syller J, 2012. Facilitative and antagonistic interactions between plant viruses in mixed infections. Molecular Plant Pathology 13, 204-16. doi: 10.1111/j.1364-3703.2011.00734.x.

Sylvester ES, 1956. Beet yellows virus transmission by the green peach aphid. Journal of Economic Entomology 49, 789-800. doi: 10.1093/jee/49.6.789. 
Sylvester ES, Richardson J, 1992. Aphid-borne rhabdoviruses-relationships with their vectors. In: Harris KF, ed. Advances in disease vector research, Springer New York: New York, NY. Vol 9, 313-41.

Tamada T, Goto T, Chiba I, 1969. Soybean dwarf, a new virus disease. Japanese Journal of Phytopathology 35, 282-5. doi: 10.3186/jjphytopath.35.282.

Tao X, Zhou X, Li G, 2002. The pathogenicity on legumes of Cucumber mosaic virus was determined by 243 nucleotides on 2a polymerase gene of viral RNA2. Chinese Science Bulletin 47, 748. doi: 10.1360/02tb9169.

Thompson GA, Goggin FL, 2006. Transcriptomics and functional genomics of plant defence induction by phloem-feeding insects. Journal of experimental botany $57,755-66$. doi: 10.1093/jxb/erj135.

Thompson JR, Dasgupta I, Fuchs M et al., 2017. ICTV virus taxonomy profile: Secoviridae. The Journal of General Virology 98, 529-31. doi: 10.1099/jgv.0.000779.

Timchenko T, Katul L, Aronson M et al., 2006. Infectivity of nanovirus DNAs: induction of disease by cloned genome components of Faba bean necrotic yellows virus. The Journal of General Virology 87, 1735-43. doi: 10.1099/vir.0.81753-0.

Timchenko T, Katul L, Sano Y, 2000. The master rep concept in nanovirus replication: identification of missing genome components and potential for natural genetic reassortment. Virology 274, 189-95. doi: 10.1006/viro.2000.0439.

Timchenko T, Kouchkovsky F de, Katul L, 1999. A single Rep protein initiates replication of multiple genome components of faba bean necrotic yellows virus, a single-stranded DNA virus of plants. Journal of Virology 73, 10173-82.

Tjallingii WF, 1978. Electronic recording of penetration behaviour by aphids. Entomologia Experimentalis et Applicata 24, 721-30. doi: 10.1111/j.1570-7458.1978.tb02836.x.

Tjallingii WF, 1985. Electrical nature of recorded signals during stylet penetration by aphids. Entomologia Experimentalis et Applicata 38, 177-86. doi: 10.1111/j.15707458.1985.tb03516.x.

Tjallingii WF, 1988. Electrical recording of stylet penetration activities. In: Minks AK, Harrewijn P, Helle W, eds. Aphids, their biology, natural enemies and control, Elsevier Science Publishers, 95-108.

Tjallingii WF, 1994. Sieve element acceptance by aphids. European Journal of Entomology 91, 47-52.

Tomlinson JA, Walkey DGA, 1967. The isolation and identification of rhubarb viruses occurring in Britain. Annals of Applied Biology 59, 415-27. doi: 10.1111/j.1744-7348.1967.tb04458.x.

Torrance L, Jones RAC, 1981. Recent developments in serological methods suited for use in routine testing for plant viruses. Plant Pathology 30, 1-24. doi: 10.1111/j.13653059.1981.tb01218.x.

Tsuchida T, Koga R, Shibao H, 2002. Diversity and geographic distribution of secondary endosymbiotic bacteria in natural populations of the pea aphid, Acyrthosiphon pisum. Molecular Ecology 11, 2123-35. doi: 10.1046/j.1365-294X.2002.01606.x. 
Tzanetakis IE, Martin RR, 2008. A new method for extraction of double-stranded RNA from plants. Journal of Virological Methods 149, 167-70. doi: 10.1016/j.jviromet.2008.01.014.

Untergasser A, Cutcutache I, Koressaar T et al., 2012. Primer3--new capabilities and interfaces. Nucleic Acids Research 40, e115. doi: 10.1093/nar/gks596.

Uzest M, Gargani D, Drucker M et al., 2007. A protein key to plant virus transmission at the tip of the insect vector stylet. Proceedings of the National Academy of Sciences of the United States of America 104, 17959-64. doi: 10.1073/pnas.0706608104.

Vainio EJ, Chiba S, Ghabrial SA et al., 2018. ICTV virus taxonomy profile: Partitiviridae. The Journal of General Virology 99, 17-8. doi: 10.1099/jgv.0.000985.

van den Heuvel JF, Verbeek M, van der Wilk F, 1994. Endosymbiotic bacteria associated with circulative transmission of potato leafroll virus by Myzus persicae. The Journal of General Virology 75 (Pt 10), 2559-65. doi: 10.1099/0022-1317-75-10-2559.

van den J. M. Heuvel JF, 1991. Transmission of potato leafroll virus from plants and artificial diets by Myzus persicae. Phytopathology 81, 150. doi: 10.1094/Phyto-81-150.

van Dijk EL, Auger H, Jaszczyszyn Y, 2014a. Ten years of next-generation sequencing technology. Trends in Genetics 30, 418-26. doi: 10.1016/j.tig.2014.07.001.

van Dijk EL, Jaszczyszyn Y, Thermes C, 2014b. Library preparation methods for next-generation sequencing: tone down the bias. Experimental Cell Research 322, 12-20. doi: 10.1016/j.yexcr.2014.01.008.

van Leur JAG, Kumari SG, 2011. A survey of lucerne in northern New South Wales for viruses of importance to the winter legume industry. Australasian Plant Pathology 40, 180-6. doi: 10.1007/s13313-011-0028-z.

van Leur JAG, Kumari SG, Aftab M, 2013. Virus resistance of Australian pea (Pisum sativum) varieties. New Zealand Journal of Crop and Horticultural Science 41, 86-101. doi: 10.1080/01140671.2013.781039.

Vemulapati B, Druffel KL, Eigenbrode SD, 2010. Molecular characterization of pea enation mosaic virus and bean leafroll virus from the Pacific Northwest, USA. Archives of Virology 155, 1713-5. doi: 10.1007/s00705-010-0767-0.

Verbeek M, Dullemans A, van den Heuvel H, 2010. Tomato chocolàte virus: a new plant virus infecting tomato and a proposed member of the genus Torradovirus. Archives of Virology 155, 751-5. doi: 10.1007/s00705-010-0640-1.

Verbeek M, Dullemans AM, van Bekkum PJ, 2013. Evidence for lettuce big-vein associated virus as the causal agent of a syndrome of necrotic rings and spots in lettuce. Plant Pathology 62, 444-51. doi: 10.1111/j.1365-3059.2012.02645.x.

Verbeek M, Dullemans AM, van den Heuvel JFJM, 2007. Identification and characterisation of tomato torrado virus, a new plant picorna-like virus from tomato. Archives of Virology 152, 881-90. doi: 10.1007/s00705-006-0917-6.

Verbeek M, Dullemans AM, van der Vlugt RAA, 2017. Aphid transmission of Lettuce necrotic leaf curl virus, a member of a tentative new subgroup within the genus Torradovirus. Virus Research 241, 125-30. doi: 10.1016/j.virusres.2017.02.008. 
Vetten HJ, Dale JL, Grigoras I, 2011. Family: Nanoviridae. In: King AMQ, Lefkowitz E, Adams MJ, Carstens EB, eds. Virus taxonomy: ninth report of the International Committee on Taxonomy of Viruses, Elsevier, 395-404.

Vetten HJ, Gronenborn B, Bressan A, 2016. Aphid transmission of viruses of the family Nanoviridae. In: Brown JK, ed. Vector-mediated transmission of plant pathogens, APS Press: St. Paul Minnesota, 453-62.

Vetten HJ, Knierim D, Rakoski MS et al., 2019. Identification of a novel nanovirus in parsley. Archives of Virology 164, 1883-7. doi: 10.1007/s00705-019-04280-3.

Villamor DEV, Ho T, Rwahnih M al, 2019. High throughput sequencing for plant virus detection and discovery. Phytopathology 109, 716-25. doi: 10.1094/PHYTO-07-18-0257-RVW.

Villamor DEV, Pillai SS, Eastwell KC, 2017. High throughput sequencing reveals a novel fabavirus infecting sweet cherry. Archives of Virology 162, 811-6. doi: 10.1007/s00705-016-3141-z.

Visser M, Bester R, Burger JT, 2016. Next-generation sequencing for virus detection: covering all the bases. Virology Journal 13, 85. doi: 10.1186/s12985-016-0539-x.

Vodovar N, Goic B, Blanc H, 2011. In silico reconstruction of viral genomes from small RNAs improves virus-derived small interfering RNA profiling. Journal of Virology 85, 11016-21. doi: 10.1128/JVI.05647-11.

Vunsh RON, Rosner A, Stein A, 1990. The use of the polymerase chain reaction (PCR) for the detection of bean yellow mosaic virus in gladiolus. Annals of Applied Biology 117, 561-9. doi: 10.1111/j.1744-7348.1990.tb04822.x.

Walker PJ, Blasdell KR, Calisher CH et al., 2018. ICTV virus taxonomy profile: Rhabdoviridae. The Journal of General Virology 99, 447-8. doi: 10.1099/jgv.0.001020.

Walker PJ, Dietzgen RG, Joubert DA, 2011. Rhabdovirus accessory genes. Virus Research 162, 110-25. doi: 10.1016/j.virusres.2011.09.004.

Walker PJ, Firth C, Widen SG et al., 2015. Evolution of genome size and complexity in the Rhabdoviridae. PLOS Pathogens 11, e1004664. doi: 10.1371/journal.ppat.1004664.

Wanitchakorn R, Hafner GJ, Harding RM, 2000. Functional analysis of proteins encoded by banana bunchy top virus DNA-4 to -6. The Journal of General Virology 81, 299-306. doi: 10.1099/0022-1317-81-1-299.

Ward E, Foster SJ, Fraaije BA, 2004. Plant pathogen diagnostics: immunological and nucleic acidbased approaches. Annals of Applied Biology 145, 1-16. doi: 10.1111/j.17447348.2004.tb00354.x.

Watanabe S, Borthakur D, Bressan A, 2013. Lack of evidence for an interaction between Buchnera GroEL and banana bunchy top virus (Nanoviridae). Virus Research 177, 98-102. doi: 10.1016/j.virusres.2013.06.002.

Watanabe S, Borthakur D, Bressan A, 2016. Localization of Banana bunchy top virus and cellular compartments in gut and salivary gland tissues of the aphid vector Pentalonia nigronervosa. Insect Science 23, 591-602. doi: 10.1111/1744-7917.12211. 
Watanabe S, Bressan A, 2013. Tropism, compartmentalization and retention of banana bunchy top virus (Nanoviridae) in the aphid vector Pentalonia nigronervosa. The Journal of General Virology 94, 209-19. doi: 10.1099/vir.0.047308-0.

Watson MA, Roberts FM, 1939. A comparative study of the transmission of hyoscyamus virus 3, potato virus $Y$ and cucumber virus 1 by the vectors Myzus persicae (Sulz), M. circumflexus (Buckton), and Macrosiphum gei (Koch). Proceedings of the Royal Society of London. Series B Biological Sciences 127, 543-76. doi: 10.1098/rspb.1939.0039.

Webster CG, Wylie SJ, Jones MGK, 2004. Diagnosis of plant viral pathogens. Current Science 86, 1604-7.

Wei T-Y, Yang J-G, Liao F-R et al., 2009. Genetic diversity and population structure of rice stripe virus in China. The Journal of General Virology 90, 1025-34. doi: 10.1099/vir.0.006858-0.

Wilkinson TL, Douglas AE, 1998. Host cell allometry and regulation of the symbiosis between pea aphids, Acyrthosiphon pisum, and bacteria, Buchnera. Journal of Insect Physiology 44, 629-35. doi: 10.1016/S0022-1910(98)00030-4.

Wool D, Hales DF, 1996. Previous infestation affects recolonization of cotton by Aphis gossypii: induced resistance or plant damage? Phytoparasitica 24, 39-48. doi: 10.1007/BF02981452.

Wu L-P, Yang T, Liu H-W, 2018. Molecular characterization of a novel rhabdovirus infecting blackcurrant identified by high-throughput sequencing. Archives of Virology, 1363-6. doi: 10.1007/s00705-018-3709-x.

Wu MD, Zhang L, Li GQ, 2007. Hypovirulence and double-stranded RNA in Botrytis cinerea. Phytopathology 97, 1590-9. doi: 10.1094/PHYTO-97-12-1590.

Wu Q, Ding S-W, Zhang Y, 2015. Identification of viruses and viroids by next-generation sequencing and homology-dependent and homology-independent algorithms. Annual Review of Phytopathology 53, 425-44. doi: 10.1146/annurev-phyto-080614-120030.

Wyant PS, Strohmeier S, Schäfer B et al., 2012. Circular DNA genomics (circomics) exemplified for geminiviruses in bean crops and weeds of northeastern Brazil. Virology 427, 151-7. doi: 10.1016/j.virol.2012.02.007.

Xie J, Ghabrial SA, 2012. Molecular characterization of two mitoviruses co-infecting a hypovirulent isolate of the plant pathogenic fungus Sclerotinia sclerotiorum corrected. Virology 428, 77-85. doi: 10.1016/j.virol.2012.03.015.

Yanagisawa $\mathrm{H}$, Tomita $\mathrm{R}$, Katsu $\mathrm{K}$ et al., 2016. Combined DECS analysis and next-generation sequencing enable efficient detection of novel plant RNA viruses. Viruses 8, 70. doi: $10.3390 / v 8030070$.

Zerbino DR, Birney E, 2008. Velvet: algorithms for de novo short read assembly using de Bruijn graphs. Genome Research 18, 821-9. doi: 10.1101/gr.074492.107.

Zhao K, Margaria P, Rosa C, 2016. First report of white clover mosaic virus and turnip mosaic virus mixed infection on garlic mustard in Pennsylvania. Plant Disease 100, 866. doi: 10.1094/PDIS-09-15-1083-PDN. 
Zheng Y, Gao S, Padmanabhan C et al., 2017. VirusDetect: an automated pipeline for efficient virus discovery using deep sequencing of small RNAs. Virology 500, 130-8. doi:

10.1016/j.virol.2016.10.017.

Zhou GH, Rochow WF, 1984. Differences among five stages of Schizaphis graminum in transmission of a barley yellow dwarf luteovirus. Phytopathology 74, 1450-3.

Ziebell H, 2015. Kleines Virus - großer Schaden. ForschungsReport spezial Ökologischer Landbau 4, 6-7.

Ziebell H, 2017. Die Virusepidemie an Leguminosen 2016 - eine Folge des Klimawandels? Journal für Kulturpflanzen 69, 64-8. doi: 10.1399/JfK.2017.02.09.

Ziebell H, Carr JP, 2010. Cross-protection. In: Carr JP, Loebenstein G, eds. Natural and Engineered Resistance to Plant Viruses, Part II, Elsevier. Vol 76, 211-64.

Ziebell H, Murphy AM, Groen SC et al., 2011. Cucumber mosaic virus and its $2 b$ RNA silencing suppressor modify plant-aphid interactions in tobacco. Scientific Reports 1, 187. doi: 10.1038/srep00187. 


\section{Acknowledgments}

"Alhamdulillah" All praise is due to Allah.

Allah said:

$$
\begin{aligned}
& \text { بِسْمِ اللَّهِ الرَّحمَحَنِ الرَّحِيمِ }
\end{aligned}
$$

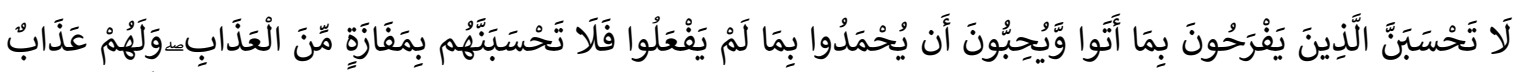

$$
\begin{aligned}
& \text { أَلْيمٌ (188) }
\end{aligned}
$$

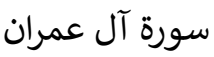

In the name of Allah, the Entirely Merciful, the Especially Merciful

And never think that those who rejoice in what they have perpetrated and like to be praised for what they did not do - never think them [to be] in safety from the punishment, and for them is a painful punishment (188)

(Sura Aal-i-Imraan)

Moreover, following the guidance of prophet Muhammad (peace and blessings of

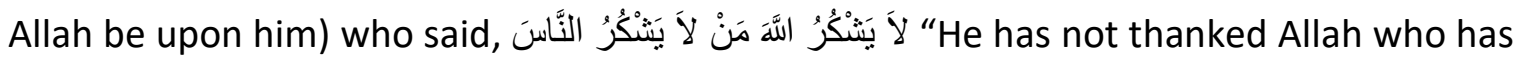
not thanked the people." (reported by Abu Huraira, source Sunan Abī Dāwūd: 4811 (authentic)). Thus, this part of the thesis is very important as every person mentioned here for me.

Although the PhD is an individual work, it is not indefinitely on your own. Many people contributed significantly to this work. Special thanks to Dr. Heiko Ziebell, Prof. Dr. Stefan Vidal and Prof. Dr. Edgar Maiss for accepting me as a PhD student and for their confidence in me. Thank you for your mentoring, guidance and patience. Dr. Heiko, you supported and stimulated me, and gave me many opportunities to improve my skills and knowledge. You are a great supervisor who stands beside his student and fully support him. To Prof. Dr. Stefan Vidal for your positive critics and valuable discussions during my work and all support specially the administrative work. I would like to thank Prof. Dr. Edgar Maiss for his advice and his critical reading for the thesis. I would like to thank Prof. Michael Rostás for accepting to be in the examination committee.

I am also grateful to Angelika Sieg-Müller, Petra Lüddecke, Kerstin Herz and Jonas Hartrick for their excellent technical assistance and for the great working atmosphere during my research period. Special thanks to all the co-author of the different publications. Many thanks to Prof. Dr. Edger Schliephake who taught me the EPG method, Dr. Katja Richert-Pöggeler for teaching me the EM for virus detection and for the valuable discussions, and to Prof. Dr. Stefan Winter and Dr. Dennis Knierim for training me to the ribodepletion approach for HTS virus detection. To Prof. Thomas Kühne and Dr. Wolfgang Maier for the valuable discussions. 
My friend Amjad Zia, sincerely you are a great support for me on personal level during master's degree and specially during my PhD studies. The PhD period was full of stressful moments, and our friendly meetings were a relief "second after prayers". I cannot forget the valuable comments and suggestions to improve my work. Thank you, brother.

My father Dr. Zakaria Abdou Gaafar and my mother, thank you for raising me and giving me all the opportunities to be successful. My brothers, sisters and brother in law for your emotional support and your prayers. My grandmother (may Allah surround you with his mercy), you mean a lot to me, sorry I was not there beside you. See you in a better place my dear.

I was supported financially by a German Egyptian long-term scholarship (GERLS), thanks to all the staff involved in this scholarship. I was also partially supported by scholarships from the Gemeinschaft der Förderer und Freunde des Julius Kühn-Instituts e. V. (GFF) and Stegemann Stiftung, thank you very much for your financial support.

This research was funded by several agencies: a grant from the Federal Office of Food and Agriculture within the Euphresco network "2015-F-172: The application of NextGeneration Sequencing technology for the detection and diagnosis of non-culturable organisms: viruses and viroids"; a German-New Zealand cooperation grant from the German Federal Ministry of Food and Agriculture (BMEL) through the Federal Office for Agriculture and Food (BLE), Germany and The Royal Society of New Zealand. Additional support was from the GFF and Stegemann Stiftung.

Thank you all, I wish you all the best

Yahya Zakaria Abdou Gaafar 
Curriculum vitae 


\section{List of publications}

2019 1. Gaafar, Y.; Lüddecke, P.; Heidler C.; Hartrick, J.; Sieg-Müller, A; Hübert, C.; Wichura, A.; Ziebell, H. (2019): First report of southern tomato virus in German tomatoes. In New Dis. Rep. 40, 1. DOI: 10.5197/j.2044-0588.2019.040.001.

2. Gaafar, Y.; Sieg-Müller, A.; Lüddecke, P.; Hartrick, J.; Seide, Y.; Müller, J.; Maaß, C.; Schuhmann, S.; Richert-Pöggeler, K. R.; Blouin, A.; Massart, S.; Ziebell, H. (2019): First report of natural infection of beetroot with beet soil-borne virus. In New Dis. Rep. DOI: 10.5197/j.2044-0588.2019.040.005.

3. Gaafar, Y. Z. A.; Richert-Pöggeler, K. R.; Sieg-Müller, A.; Lüddecke, P.; Herz, K.; Hartrick, J.; Seide, Y.; Vetten, H.-J.; Ziebell, H. (2019): A divergent strain of melon chlorotic spot virus isolated from black medic (Medicago lupulina) in Austria. In Virol. J. 16, 297. DOI: 10.1186/s12985-019-1195-8.

4. Gaafar, Y. Z. A.; Richert-Pöggeler, K. R.; Sieg-Müller, A.; Lüddecke, P.; Herz, K.; Hartrick, J.; Maaß, C.; Ulrich, R.; Ziebell, H. (2019): Caraway yellows virus, a novel nepovirus from Carum carvi. In Virol. J. 16, 529. DOI: 10.1186/s12985-019-1181-1.

5. Gaafar, Y. Z. A.; Ziebell, H. (2019): Complete genome sequence of highly divergent carrot torradovirus 1 strain from Apium graveolens. In Arch. Virol. DOI: 10.1007/s00705-019-04272-3.

6. Gaafar, Y. Z. A.; Richert-Pöggeler, K. R.; Maaß, C.; Vetten, H.-J.; Ziebell, H. (2019): Characterisation of a novel nucleorhabdovirus infecting alfalfa (Medicago sativa). In Virol. J. 16 (1), p. 113. DOI: 10.1186/s12985-019-1147-3.

7. Gaafar, Y. Z. A.; Ziebell, H. (2019): Two divergent isolates of turnip yellows virus from pea and rapeseed and first report of turnip yellows virus-associated RNA in Germany. In Microbiol. Resour. Announc. 8 (17), p. 2254. DOI: 10.1128/MRA.00214-19.

8. Gaafar, Y.; Sieg-Müller, A.; Lüddecke, P.; Herz, K.; Hartrick, J.; Maaß, C. Schuhmann, S.; Richert-Pöggeler, K. R.; Ziebell, H. (2019): First report of turnip crinkle virus infecting garlic mustard (Alliaria petiolata) in Germany. In New Dis. Rep. 39, p. 9. DOI: 10.5197/j.2044-0588.2019.039.009.

9. Gaafar, Y.; Cordsen Nielsen, G.; Ziebell, H. (2018): Molecular characterisation of the first occurrence of pea necrotic yellow dwarf virus in Denmark. In New Dis. Rep. 37, p. 16. DOI: 10.5197/j.2044-0588.2018.037.016.

10. Gaafar, Y. Z. A.; Abdelgalil, M. A. M.; Knierim, D.; Richert-Pöggeler, K. R.; Menzel, W.; Winter, S.; Ziebell, H. (2018): First report of physostegia chlorotic mottle virus on tomato (Solanum lycopersicum) in Germany. In Plant Dis. 102 (1), p. 255. DOI: 10.1094/PDIS-05-17-0737-PDN.

2017 11. Gaafar, Y.; Timchenko, T.; Ziebell, H. (2017): First report of pea necrotic yellow dwarf virus in The Netherlands. In New Dis. Rep. 35, p. 23. DOI: 10.5197/j.20440588.2017.035.023.

12. Gaafar, Y.; Grausgruber-Gröger, S.; Ziebell, H. (2016): Vicia faba, V. sativa and Lens culinaris as new hosts for pea necrotic yellow dwarf virus in Germany and Austria. In New Dis. Rep. 34, p. 28. DOI: 10.5197/j.2044-0588.2016.034.028. 
Conferences and meetings

Jun 2019 Poster Legume viruses

EP-colloquium, JKI

Braunschweig, Germany

Mar 2019 Presentation Plant viruses identified using high throughput sequencing

DPG meeting of plant virus diseases working group, Göttingen, Germany

Sep 2018 Presentation Comparative study on three RNAEuphresco meeting, NPPO based pipelines for plant Wageningen, The Netherlands virus/viroid detection using high throughput sequencing

Sep 2018 Presentation EUPHRESCO project (2015-F-172) report

Euphresco meeting, NPPO Wageningen, The Netherlands

Aug 2018 Poster Plant virus detection and EP-Minisymposium, JKI identification, and virus-vectorhost interactions

Aug 2018 Poster Legume viruses braunschweig, Germany

EP-Minisymposium, JKI Braunschweig, Germany

Mar 2017 Presentation Legume viruses in Germany 2016 Dutch and German Plant Virologists meeting, Bonn, Germany

Nov 2016 Presentation Insights into the Nanoviruslegume-aphid interactions

Young Scientists Meeting (YSM) JKI Quedlinburg, Germany

Oct 2016 Presentation dsRNA immunocapturing

Euphresco meeting, JKI Braunschweig, Germany

Sep 2016 Poster The current status of legume viruses in Germany

Advances in Virology conference, Association of Applied Biologists, University of Greenwich London, The UK 
